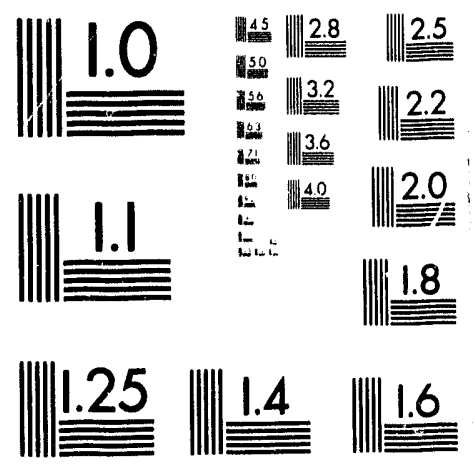



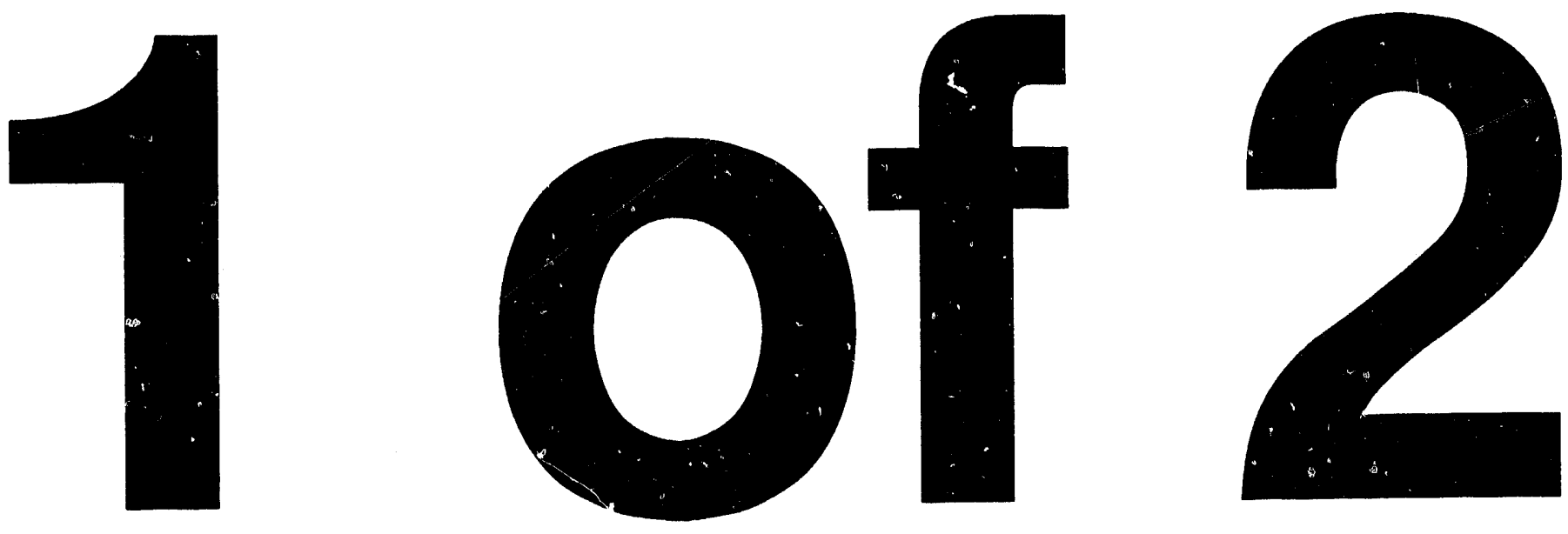


\title{
Sampling and Analyses Report for December 1992 Semiannual Postburn Sampling at the RMI UCG Site, Hanna, Wyoming
}

\author{
Topical Report
}

\section{Steven R. Lindblom}

March 1993

Work Performed Under Contract No.: DE-FG21-88MC25038

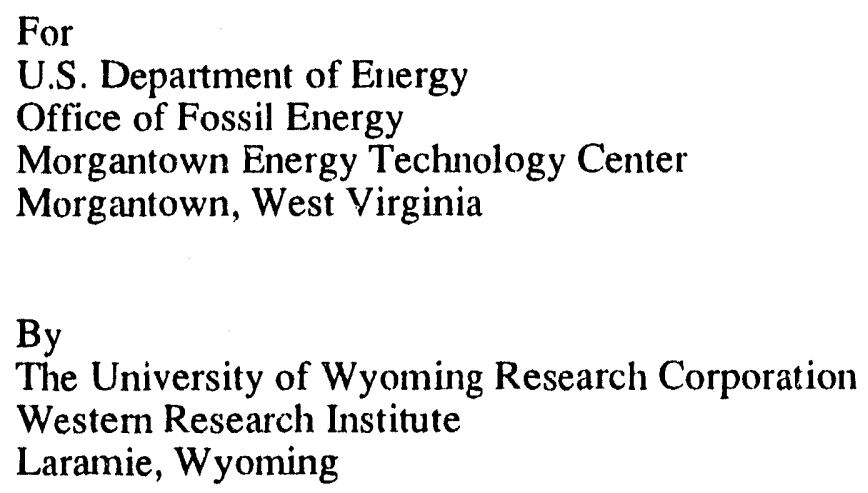

By

The University of Wyoming Research Corporation

Western Research Institute

Laramie, Wyoming

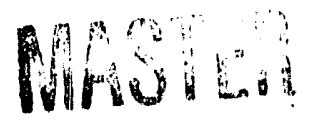




\section{DISCLAIMER}

This report was prepared as an account of work sponsored by an agency of the United States Government. Neither the United States Government nor any agency thereof, nor any of their employees, makes any warranty, express or implied, or assumes any legal liability or responsibility for the accuracy, completeness, or usefulness of any inforcation, apparatus, product, or process disclosed, or represents that its use would not infringe privately owned rights. Reference herein to any specific commercial product, process, or service by trade name, trademark, manufacturer, or otherwise does not necessarily constitute or imply its endorsement, recommendation, or favoring by the United States Government or any agency thereof. The views and opinions of authors expressed herein do not necessarily state or reflect those of the United States Govermment or any agency thereof.

This report has been reproduced directly from the best available copy.

Available to DOE and DOE - ntractors from the Office of Scientific and Technical Information, P.O. Box 62, Oak Ridge, TN 37831; prices available at (6.15) $576-8401$.

Available to the public from the National Technical Information Service, U.S. Department of Commerce, 5285 Port Royal Rd., Springfield, VA 22161; phone orders accepted at (703) 487-4650. 


\title{
Sampling and Analyses Report for December 1992 Semiannual Postburn Sampling at the RMI UCG Site, Hanna, Wyoming
}

\author{
Topical Report
}

Steven R. Lindblom

Work Ptringriset Under Contract No.: DE-FG21-88MC25038

\author{
For \\ U.S. Department of Energy \\ Office of Fossil Energy \\ Morgantown Energy Technology Center \\ P.O. Box 880 \\ Morgantown, West Virginia 26507-0880
}

\author{
By \\ The University of Wyoming Research Corporation \\ Western Research Institute \\ P.O. Box 3395, University Station \\ Laramie, Wyoming 82071
}

March 1993 
TABLE OF CONTENTS

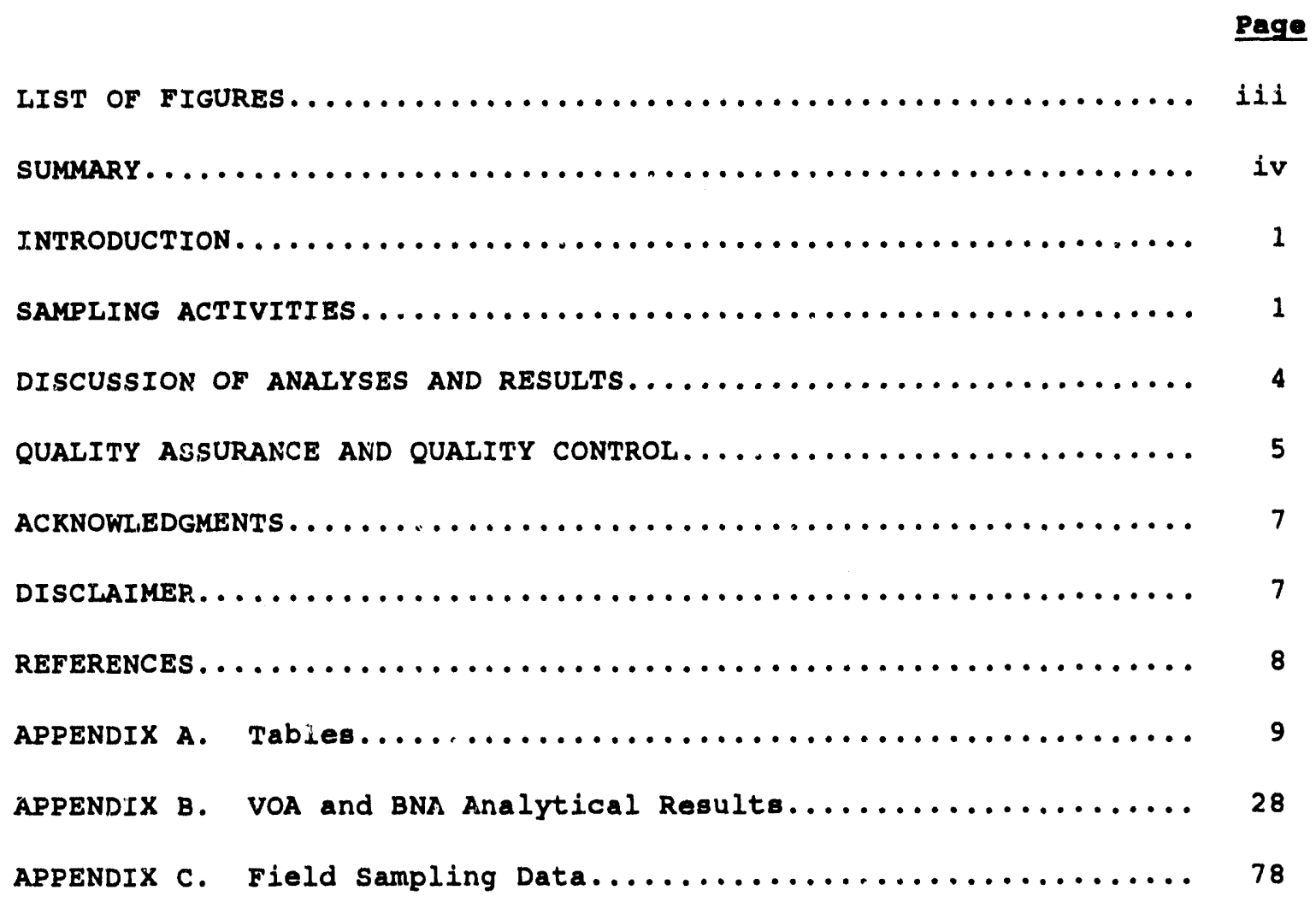




\section{Figure}

1. Location of Rocky Mountain 1 Underground Coal

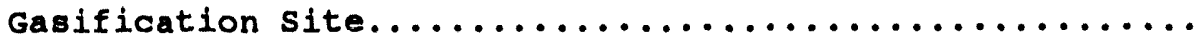

2. Potentiometric Surface Map of the Hanna No. 1 Coal Seam

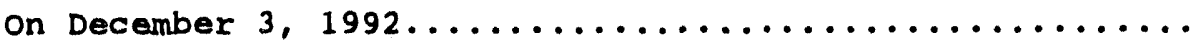




\section{gUMQLARY}

During December 1992, groundwater was sampled at the site of the November $1987-F e b r u a r y ~ 1988$ Rocky Mountain 1 underground coal gasification test near Hanna, Wyoming. The groundwater is near baseline condition. Data from the field measurements and analyses of samples are presented. Benzene concentrations in the groundwater are below analytical detection limits (<0.01 $\mathrm{mg} / \mathrm{L})$ for all wells, except concentrations of $0.016 \mathrm{mg} / \mathrm{L}$ and $0.013 \mathrm{mg} / \mathrm{L}$ in coal seam wells EMW-3 and EMW-1, respectively. 


\section{INTRODUCTION}

The Rocky Mountain 1 (RM1) underground coal gasification (UCG) test was conducted from November 16, 1987, through Fehruary 26, 1988 (United Engineers and Constructors 1989), at a site approximately one mile south of Hanna, wyoming (Figure 1). The test consisted of a dual-module operation to evaluate the controlled retracting injection point (CRIP) technology, the elongated linked well (ELW) technology, and the interaction of closely spaced modules operating simultaneously. The test caused two cavities to form in the Hanna No. 1 coal seam and associated overburden. The Hanna No. 1 coal seam is approximately $30 \mathrm{ft}$ thick and occurs at depths between 350 and $365 \mathrm{ft}$ below the surface in the test area. The coal seam is overlain by sandstones, siltstones, and claystones deposited by various fluvial environments. Details of the geology and hydrology were reported by oliver (1987) and Mason et al. $(1987)$.

Groundwater monitoring was designed to satisfy the requirements of the Wyoming Department of Environmental Quality (WDEQ) in addition to providing research data toward the development of UCG technology that minimizes environmental impacts. Further background material and the sampling and analytical procedures associated with the sampling task are described in the Rocky Mountain 1 Postburn Groundwater Monitoring Quality Assurance Plan (Mason and Johnson 1988).

\section{SAMPLING ACTIVITIES}

The December 1992 semiannual groundwater sampling took place from December 3 through December 10, 1992. Samples were collected for analyses of a full-suite set of parameters (Table 1) following the schedule in Table 2. (Tables are located in Appendix A.) Adverse weather conditions caused several problems during this sampling event. Temperatures as $10 \mathrm{w}$ as $-40^{\circ} \mathrm{F}\left(-40^{\circ} \mathrm{C}\right)$ caused sample lines and preservatives to freeze, and several volatile organic samples were lost (TW16-A, TW16-Adup, TW15-Adup, and TW17-Adup). Wind speeds up to $75 \mathrm{mph}$ combined with 6-7 inches of snow caused significant drifting and prevented site access on December 5 and 6 .

Water levels were measured across the site before any sampling activities were begun. Water table elevations in the coal seam aquifer rose approximately 2 ft since June 1992. Water level elevations measured on December 3, 1992, are listed in Table 3. A map of the potentiometric surface of the Hanna No. 1 coal seam (Figure 2) shows a hydraulic gradient from the southeast corner of the site to the northwest corner with a maximum elevation change of about $6 \mathrm{ft}$.

A total of 20 of the specified 22 wells were sampled during the December 1992 semiannual sampling. No sample was collected from unit $C$ overburden well EMW-4 because of an obstruction in the well bore at a depth of approximately $115 \mathrm{ft}$. This obstruction was first encountered during the December 1990 quarterly sampling. This deletion was discussed with WDEQ at the May 23, 1991, Technical Advisory subcommittee (TASC) meeting. CRIP cavity well CCW-1 was sampled in place of cavity 


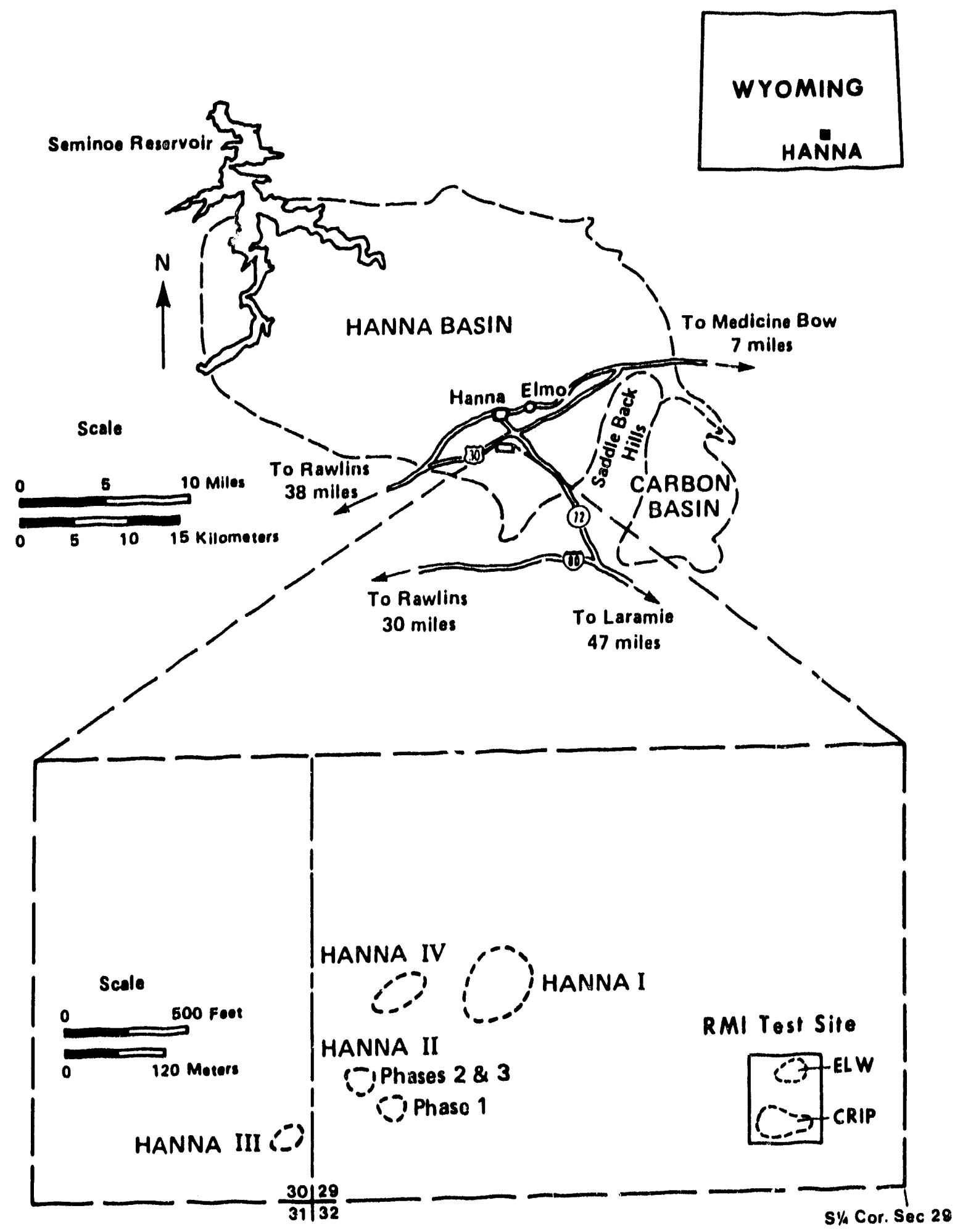

Figure 1. Location of Rocky Mountain 1 Underground Cosl Gasification site 


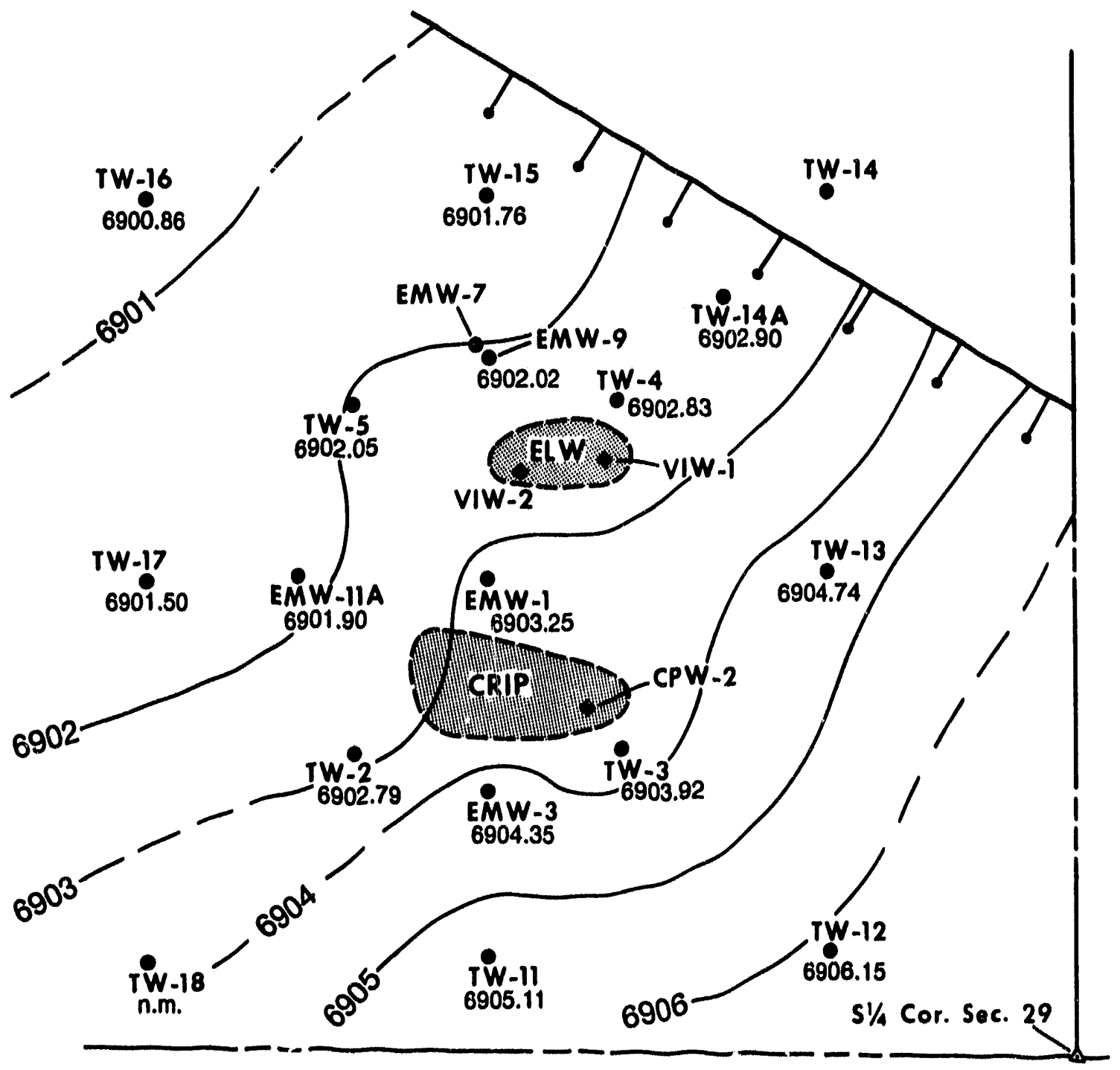

\section{Legend}
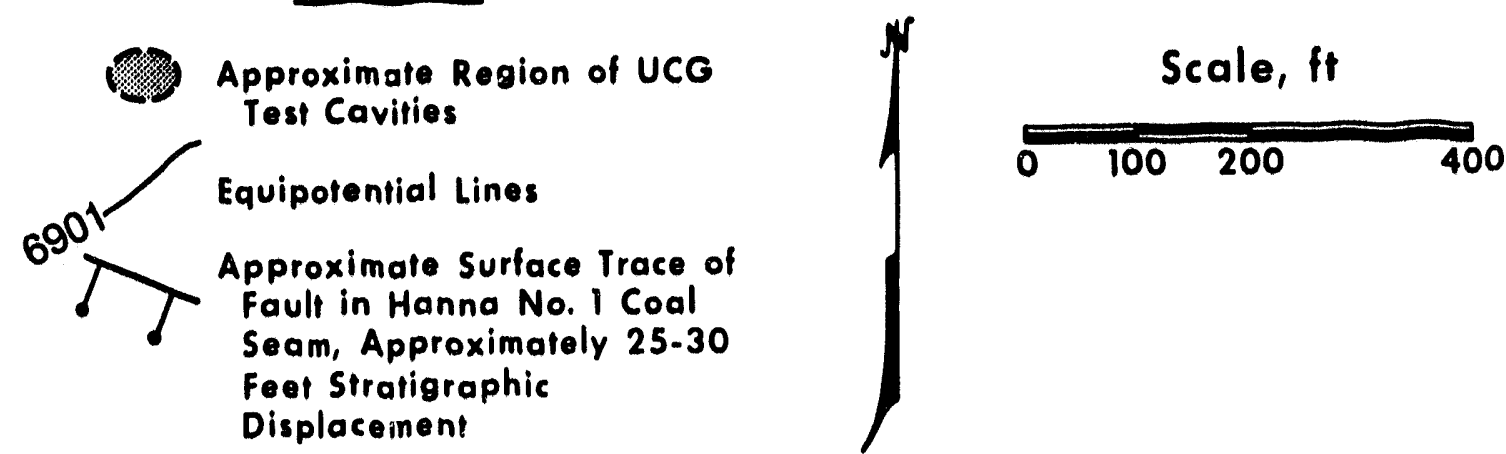

Figure 2. Potentiometric surface Map of the Banna Mo. 1 Cosl seam on Leconber 3, 1992 
well CPW-2 because roof collapse in the CRIP cavity caused the casing to shift in CPW-2 (Lindblom 1990). This substitution was approved by WDEQ at the November 28, 1990, TASc moeting. An obstruction was encountered at a depth of $240 \mathrm{ft}$ when lowering the sampling pump in well cCW-1. After several attempts, the sampling pump was successfully lowered to $285 \mathrm{ft}$. This depth is approximately $20 \mathrm{ft} 10 \mathrm{ss}$ than the depth that was attained in previous sampling events, possibly indicating further borehole collapse.

Three quality assurance/quality control samples were submitted to check sampling and analytical accuracy. All wells were sampled for the full suite (FS) of parameters, which are listed in Table 1.

The sampling activities performed by Western Research Institute (WRI) at the RM1 site during December 1992 are summarized in Table 4. This list shows the wells sampled, sampling date, sampling method, sample parameter suite, and observations made during sampling.

The wells were purged before sample collection to ensure that the samples were obtained from the hydrostratigraphic unit and not from the well bore. Stabilization of purging parameters (purge time, temperature, conductivity, $\mathrm{pH}, \mathrm{Eh}$, water level, and pump discharge rate) was used to indicate formation water recovery. As required by WDEQ, wells were purged a minimum of 80 minutes at approximately $1.0 \mathrm{gal} / \mathrm{min}$ to ensure sampling of formation water. Flow rates were reduced only after 80 minutes of pumping to obtain a ample. An exception was well EMW-10, where the water level in the well bore dropped below the pump after only 60 minutes. The flow rate was reduced immediately after the water level dropped below the pump intake. Data were collected at 20minute intervals during well purging and recorded. Well purging data are listed in Appendix C.

Also included in Appendix $C$ is the WRI groundwater sampling record form. This form includes general sampling information, stabilized purging parameter data, field alkalinity titration data, and analytical submissions. Appendix $C$ contains these data for each well sampled.

\section{DISCU88ION OF ANALYSES AND RESULTS}

Evaluation of the analytical data shows that most parameters are consiscent with concentrations detected in previous sampling events. Results from the full-suite analysis of cavity, coal seam, and overburden wells are listed in Tables 5,6 and 7 , respectively. A comparison of these analytical results with baseline data (Table 8 ) shows that groundwater at the site is at or near baseline conditions. Analytical methods are listed in Table 9.

Benzene concentrations at the RM1 site in December 1992 were below detection limits $(<0.01 \mathrm{mg} / \mathrm{L})$, except in coal seam wells $\mathrm{EMW}-1$ and $\mathrm{EMW}-$ 3. Well EMW-1 has shown benzene concentrations that range from below detection limits to a high in recent years of $0.044 \mathrm{mg} / \mathrm{L}$. Over the past year, benzene concentrations in EMW-1 have decreased slightly from 0.021 $\mathrm{mg} / \mathrm{L}$ in June 1992 to $0.013 \mathrm{mg} / \mathrm{L}$ in December 1992. Benzene 
concentrations in EMW-3 have remained relatively stable. Results f:om June 1992 and December 1992 show benzene concentrations in EMW-3 of $0.019 \mathrm{mg} / \mathrm{L}$ and $0.016 \mathrm{mg} / \mathrm{L}$, respectively. rolatile organics analysis data sheets are included in Appendix $B$.

The acetone detected in voletile organics analysis (VOA) samples from roal seam wells $\mathrm{TW}-15$ and $\mathrm{TW}-1 \mathrm{~T}$ has not been measured at the site in recent years. Discussions with analysts at WRI suggest that the souxce of the acetone is probably something other than UCG contaminants. Acetone is a very common and pervasive solvent with a very high background detection limit set by the U.S. Environmental protection Agency (EPA) $(0.050 \mathrm{mg} / \mathrm{L})$. The acetone concentrations measured in samples from $\mathrm{TW}-15$ and $\mathrm{TW}-17(0.038$ and $0.015 \mathrm{mg} / \mathrm{L}$, respectively) may be the result of something as insignificant as a heater turning on in the laboratory during the analyses.

The highest baseline concentration (HBC) was established for some constituents prior to gasification operations at the RMI site. These baseline conceitrations are based on the highest concentrations measured in four sampling events over one year. Concentrations greater than the HBC of total organic carbon (TOC), total dissolved solide (TDE), and ammonia have been measured consistently in coal seam welis in the eastern section of the Ril site. These high concentrations probably result from an influx of water from off-site rather than from UCG operations (Lindblom and Covell 1991). In December 1992, coal seam groundwater exceeded HBC levels of TOC only in well TW-2. HBCs for ammonia were not exceeded in any coal seam wells. HBCs for TDS in coal seam wells were only exceeded in cavity well VIW-1. Of the overburden wells, EMW-2 and EMW-8 exceeded overburden HBCE for TDS, EMW-2 exceeded HBCs for ammonia, and EMW-2 exceeded HBCs for TOC (Table 10).

Boron concentrations in the CRIP and the ELW cavities have also exceeded baseline levels consistentiy. Table 10 shows boron concentrations in the two cavity wells, VIW-1 and CCW-1. However, boron concentrations have exceeded the HBC in noncavity coal seam wells on only four occasione since postburn sampling started. Boron concentrations have not exceeded the HBC by more than $0.01 \mathrm{mg} / \mathrm{L}$ in any of the noncavity coal seam wells at any time during the RMl study.

The values obtained for the full-suite parameters in December 1992 are comparable to baseline values (Table 8) obtained prior to gasification operations. Except for the instances noted above, the groundwater is near baseline conditions.

\section{QUALITY ASSURANCE AND QUALITY CONTROL}

The results of the quality assurance and quality control (QA/QC) standard, duplicate, and blank samples are listed in Table 11. Table 11 also liats the expected results for each sample as determined by WRT's QA/QC officer, Dr. John MCKay. The expected results for the duplicate sample were the results obtained from the regular sample of well EMW-1. Expected results for the blank sample vere below analytical detection limits. 
Acceptable variations from expected values are specific to each parameter. When the actual result is near or outside the acceptable limit, the parameter is reexamined to determine the cause.

Concentrated stock solutions were supplied by the WRI QA/QC officer and submitted as field standard samples. Field standards were designed to check analytical accuracy and the effects of transportation and storage on samples of known concentrations. Standards of each eleinent or compound to be analyzed were submitted anonymously within the regular sample set according to the instructions of the QA/QC officer. The sample preparation and analytical procedures were also suppliea by the QA/QC officer.

Thity-nine reported analyses for field standards were within acceptable limits. Only the analysis of the selenium standard was questionable. A value of less than $0.1 \mathrm{mg} / \mathrm{L}$ was reported, and the expected value was $0.120 \mathrm{mg} / \mathrm{L}$. The analyst informed the $Q A$ officer that a value of $0.1 \mathrm{mg} / \mathrm{L}$ is the detection limit for the method. The reported value is therefore within acceptable limits.

A field duplicate sample was collected from well EMW-1, which has been one of the most contaminated wells at the RMl site. The regular and duplicate samples were prepared and preserved in an identical manner. The duplicate sample was submitted anonymously using a predetermined name. Field duplicates were designed to check analytical accuracy and precision. All values for the duplicate sample fell within an acceptable range of accuracy and precision.

Rinsate sampling is intended to simulate the rinsing that occurs while purging each well before sampling. For this reason, the amount of rinsate water pumped through the sampling system for rinsate sampling represents an average amount of water pumped from each well.

Seventy-five gallons of local tap water was pumped through the sampling system. The line was then evacuated using compressed air. Finally, 10 gallons of Type I water was pumped through the line and sampled. Type I water is high-purity, deionized, distilled water prepared in WRI's analytical laboratory using distilled water as a feedstock and processing it through a Millipore water purification system. The Millipore system recirculates water through four cartridges (one carbon adsorption, one particulate trap, and two ion exchanges) and has a conductivity of less than $2 \mu \mathrm{s} / \mathrm{cm}$. The Type I water is, thus, assumed to be blank, and any level of parameters detected in the blank samples is assumed to be the result of cross-contamination from the sample line. The small concentrations of some parameters detected in the rinsate samples may be due to incomplete rinsing of the tap water in the sample line by the Type I water. 


\section{ACKNOWLEDGMENTS}

This work was supported by the U.S. Department of Energy under grant DE-FG21-88MC25038 and by the Gas Research Institute under contract 5087253-1619.

\section{DISCLAIMER}

Mention of specific brand names or models of equipment is for information only and does not imply endorsement of any particular brand. 
Lindblom, S.R., 1990, Sampling and Analysis Report fo: September 1990 Quarterly Postburn sampling at the RM1 UCG site, Hanna, Wyoming. WRI report to DOE, Larmie, WY, WRI-90-R041.

Iindblom, S.R., and J.R. Covell, 1991, Western Research Institute Annual Technical Progress Report January - December 1990 Rocky Mountain 1 Underground Coal Gasification Project. Unpubiished WRI report to United Engineers and Constructors Inc.

Mason, J.M. and L.S. Johnson, 1988 , Rocky Mountain 1 Postburn Groundwater Monitoring Quality Assurance Plan. Laramie, WY, unpublished WRI report to DOE and GRI, WRI-91-R011.

Mason, J.M., R.L. Oliver, J.D. Schrieber, C. Moody, P. Smith, and M.J. Healy, 1987, Volume 1: Geohydrology of the Proposed Rocky Mountain 1 UCG Site, Hanna, Wyoming. WRI report to DOE and GRI, Laramie, WY, WRI-90-R056.

Oliver, R.L., 1987, Geologic Evaluation of the Proposed Rocky Mountain I UCG Test site, Hanna, Wyoming. WRI report to DOE, Laramie, WY, DOE Report DOE/MC/11076-2312.

United Engineers and Constructors Inc., Stearns-Roger Div., 1989, Rocky Mountain 1 Underground Coal Gasification Test, Hanna, Wyoming, Summary Report, Volume 1, Operations. Stearns-Roger Project Report. 
APPENDIX $\mathrm{A}$

Tables 


\section{CONTENTS OF APPENDIX A}

Table

Page

1. Rocky Mountain 1 Groundwater Analysis suites............. 11

2. Rocky Mountain 1 Groundwater Restoration Monitoring schedule........................ 12

3. Water Level Elevations at the RMI site on December 3, 1992.. 13

4. RM1 sampling summary for December $1992 \ldots \ldots \ldots \ldots \ldots \ldots \ldots$

5. Analyses for Samples obtained from cavity wells.......... 16

6. Analyses for Samples obtained from Coal seam Wells........ 17

7. Analyses for samples obtained from overburden Wells........ 21

8. Baseline Groundwater Parameters at the RM1 UCG Project,

Hanna, wyoming............................ 22

9. Summary of Methods and Accuracy, Precision, and Detection-Limit objectives..................... 23

10. Wells Exceeding Highest Baseline Concentrations of Indicator Constituentr, June 1992 (all units are $\mathrm{mg} / \mathrm{L}$ )... 25

11. Analyses for Quality Assurance/Quality Control Samples..... 26

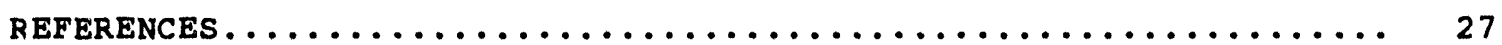


Table 1. Rocky Mountain 1 Groundwater Analys is suites

\begin{tabular}{|c|c|c|c|}
\hline $\begin{array}{l}\text { Compliance } \\
\text { suite }\end{array}$ & $\begin{array}{l}\text { Iimited } \\
\text { suite }\end{array}$ & $\begin{array}{l}\text { Full } \\
\text { suite }\end{array}$ & $\begin{array}{c}\text { Field } \\
\text { Measurements }\end{array}$ \\
\hline $\begin{array}{l}\text { Ammonia } \\
\text { Boron } \\
\text { Cyanide } \\
\text { Phenole } \\
\text { Sulfate } \\
\text { Sulfide } \\
\text { TDS } \\
\text { TOC }\end{array}$ & $\begin{array}{l}\text { Ammonia } \\
\text { Bicarbonate } \\
\text { Boron } \\
\text { Cyanide } \\
\text { Fluoride } \\
\text { Manganese } \\
\text { Nitrate } \\
\text { Nitrite } \\
\text { Phenols } \\
\text { Sodium } \\
\text { Sulfate } \\
\text { Sulfide } \\
\text { TDS } \\
\text { TKN } \\
\text { TOC }\end{array}$ & $\begin{array}{l}\text { Alkalinity } \\
\text { Aluminum } \\
\text { Ammonia } \\
\text { Aruenic } \\
\text { Barium } \\
\text { Bicarbonate } \\
\text { BNA } \\
\text { Boron } \\
\text { Bromide } \\
\text { Cadmium } \\
\text { Calcium } \\
\text { Carbonat } \\
\text { Chemical oxygen Demand } \\
\text { Chloride } \\
\text { Chromium } \\
\text { Copper } \\
\text { Cyanide } \\
\text { Fluoride } \\
\text { Iron } \\
\text { Lead } \\
\text { Lithium } \\
\text { Magnesium } \\
\text { Manganese } \\
\text { Mercury } \\
\text { Molybdenum } \\
\text { Nickel } \\
\text { Nitrate } \\
\text { Nitrite } \\
\text { pH } \\
\text { Phenols } \\
\text { Potassium } \\
\text { Selenium } \\
\text { Silver } \\
\text { Sodium } \\
\text { Sulfate } \\
\text { Sulfide } \\
\text { TDs } \\
\text { TKN } \\
\text { Thiocyanate } \\
\text { ToC } \\
\text { Volatile organics } \\
\text { Vanadium } \\
\text { Zinc }\end{array}$ & $\begin{array}{l}\text { Alkalinity } \\
\text { Conductivity } \\
\text { Discharge Rate } \\
\text { Eh } \\
\text { pH } \\
\text { Pumping Time } \\
\text { Temperature } \\
\text { Water Level }\end{array}$ \\
\hline
\end{tabular}


Table 2. Rocky Mountain 1 Croundwater Reatoration Monitoring sobedule

\begin{tabular}{|c|c|c|c|c|}
\hline Year & Quarter & $\begin{array}{c}\text { Inner-Ring } \\
\text { Wells }\end{array}$ & $\begin{array}{c}\text { Outer-Ring } \\
\text { Wells }\end{array}$ & $\begin{array}{l}\text { Cavity } \\
\text { Wells }\end{array}$ \\
\hline 1988 & $\begin{array}{l}\text { 1. March } \\
\text { 2. June } \\
\text { 3. September } \\
\text { 4. December }\end{array}$ & $\begin{array}{l}\text { Fs } \\
\mathrm{CS} \\
\mathrm{CS} \\
\mathrm{CS}\end{array}$ & $\begin{array}{l}\text { LS } \\
\text { CS } \\
\text { CS } \\
\text { CS }\end{array}$ & $\begin{array}{l}\text { FS } \\
\text { FS } \\
\text { CS } \\
\text { CS }\end{array}$ \\
\hline 1989 & $\begin{array}{l}\text { 1. March } \\
\text { 2. June } \\
\text { 3. September } \\
\text { 4. December }\end{array}$ & $\begin{array}{c}\text { CS } \\
\text { CS, VOA } \\
\text { CS, VOA } \\
\text { ES }\end{array}$ & $\begin{array}{l}\text { CS } \\
\text { CS } \\
\text { CS } \\
\text { Fs }\end{array}$ & $\begin{array}{c}\text { CS } \\
\text { CS, VOA } \\
\text { CS, VOA } \\
\text { FS }\end{array}$ \\
\hline 1990 & $\begin{array}{l}\text { 1. March } \\
\text { 2. June } \\
\text { 3. September } \\
\text { 4. December }\end{array}$ & $\begin{array}{c}\text { CS, VOA } \\
\text { LS, VOA } \\
\text { CS, VOA } \\
\text { FS }\end{array}$ & $\begin{array}{l}\text { CS } \\
\text { IS } \\
\text { CS } \\
\text { FS }\end{array}$ & $\begin{array}{c}\text { CS, VOA } \\
\text { LS, VOA } \\
\text { CS, VOA } \\
\text { FS }\end{array}$ \\
\hline 1991 & $\begin{array}{l}\text { 2. June } \\
\text { 4. December }\end{array}$ & $\begin{array}{c}\text { LS, Benzene } \\
\text { FS }\end{array}$ & $\begin{array}{c}\text { LS, Benzene } \\
\text { FS }\end{array}$ & $\begin{array}{c}\text { Is, Benzene } \\
\text { FS }\end{array}$ \\
\hline 1992 & $\begin{array}{l}\text { 2. June } \\
\text { 4. December }\end{array}$ & $\begin{array}{c}\text { LS, Benzene } \\
\text { FS }\end{array}$ & $\begin{array}{c}\text { LS, Benzene } \\
\text { FS }\end{array}$ & $\begin{array}{c}\text { Is, Benzene } \\
\text { FS }\end{array}$ \\
\hline
\end{tabular}

Cs - Compliance suite

Ls - Limited suite

FS - Full suite

VOA - Volatile Organic Analysis 
Table 3. Water Level Elevations at the Ru1 site on Decenber 3, 1992

\begin{tabular}{|c|c|c|c|c|}
\hline $\begin{array}{l}\text { Well } \\
\text { Name }\end{array}$ & $\begin{array}{l}\text { well casing } \\
\text { Elevation, ft }\end{array}$ & $\begin{array}{l}\text { Water Level } \\
\text { Depth, ft }\end{array}$ & $\begin{array}{l}\text { Water Level } \\
\text { Elevation, ft }\end{array}$ & $\begin{array}{c}\text { Pretest } \\
\text { Water Level } \\
\text { Elevation , ft }\end{array}$ \\
\hline EMW-1 & 6984.8 & 81.55 & 6903.25 & 6898.8 \\
\hline EMW-2 & 6986.3 & 77.33 & 6908.97 & -- \\
\hline EMW-3 & 7006.0 & 101.65 & 6904.35 & 6899.3 \\
\hline EMW-4 & 6981.4 & 106.83 & 6874.57 & -- \\
\hline EMW-6 & 6960.7 & 31.60 & 6929.10 & -- \\
\hline EMW-8 & 6964.6 & 149.64 & 6815.16 & -- \\
\hline EMT-9 & 6961.7 & 59.68 & 6902.02 & 6897.2 \\
\hline$E M W-10$ & 6964.2 & 91.93 & 6872.27 & -- \\
\hline EMW-11a & 6980.8 & 78.90 & 6901.90 & 6897.1 \\
\hline TW-2 & 6993.9 & 93.61 & 6902.79 & 6899.2 \\
\hline TW-3 & 6998.2 & 94.28 & 6903.92 & 6899.6 \\
\hline$T W-4$ & 6964.8 & 61.97 & 6902.83 & 6897.2 \\
\hline$T W-5$ & 6968.6 & 66.55 & 6902.05 & 6895.9 \\
\hline TK-11 & 7007.6 & 102.49 & 6905.11 & 6902.0 \\
\hline$T W-12$ & 7009.2 & 103.05 & 6906.15 & 6903.9 \\
\hline TW-13 & 6978.8 & 74.06 & 6904.74 & 6900.5 \\
\hline TW-14 & 6950.7 & 71.35 & 6879.35 & 6867.0 \\
\hline$T W-14 a$ & 6956.4 & 53.50 & 6902.90 & -- \\
\hline$T W-15$ & 6967.4 & 65.64 & 6901.76 & 6895.8 \\
\hline$T W-16$ & 6986.4 & 85.54 & 6900.86 & 6893.3 \\
\hline TW-17 & 6973.6 & 72.40 & 6901.50 & 6898.1 \\
\hline TW-18 & 6995.1 & $\mathrm{n} . \mathrm{m}$. & n.m. & 6900.4 \\
\hline VIW-1 & 6968.0 & 65.50 & 6905.00 & -- \\
\hline$C C W-1$ & 6994.16 & 90.25 & 6903.95 & -- \\
\hline
\end{tabular}

arom November 13, 1987, Hanna No. 1 coal seam measurements 
rable 4. RM1 ganoling sumary for Docomber 1992

\begin{tabular}{|c|c|c|c|c|}
\hline $\begin{array}{l}\text { Well } \\
\text { Name }\end{array}$ & $\begin{array}{l}\text { Date } \\
\text { sampled }\end{array}$ & $\begin{array}{l}\text { Sample } \\
\text { Method }\end{array}$ & $\begin{array}{l}\text { Parameter } \\
\text { suite }\end{array}$ & Commente \\
\hline EMW-1 & $12 / 9 / 92$ & Bennett pump & FS & $\begin{array}{l}\text { Water is clear, } \\
\text { slight sulfur } \\
\text { odor. }\end{array}$ \\
\hline EMW-2 & $12 / 9 / 92$ & Teflon bailer & FS & $\begin{array}{l}\text { Water is clear } \\
\text { and odor free. }\end{array}$ \\
\hline EMW-3 & $12 / 9 / 92$ & Bennett pump & re & $\begin{array}{l}\text { Water is olear, } \\
\text { slight hydrocarbon } \\
\text { odor. }\end{array}$ \\
\hline EMW-8 & $12 / 8 / 92$ & Teflon bailer & F's & $\begin{array}{l}\text { Water is olear } \\
\text { and odor free. }\end{array}$ \\
\hline EMW-9 & $12 / 8 / 92$ & Bennett punp & FS & $\begin{array}{l}\text { Water is clear } \\
\text { and odor free. }\end{array}$ \\
\hline EMW-10 & $12 / 8 / 92$ & Bennett pump & FS & $\begin{array}{l}\text { Water is clear } \\
\text { and odor free. }\end{array}$ \\
\hline EMW-11a & $12 / 9 / 92$ & Bennett pump & FS & $\begin{array}{l}\text { Water is clear, } \\
\text { olight sulfur } \\
\text { odor. }\end{array}$ \\
\hline TW-2 & $12 / 9 / 92$ & Bennett pump & FS & $\begin{array}{l}\text { Water is clear, } \\
\text { slight sulfur } \\
\text { odor. }\end{array}$ \\
\hline$T W-3$ & $12 / 8 / 92$ & Bennett pump & FS & $\begin{array}{l}\text { Water is clear } \\
\text { and odor free. }\end{array}$ \\
\hline$T W-4$ & $12 / 8 / 92$ & Bennett pump & FS & $\begin{array}{l}\text { Water is clear } \\
\text { and odor free. }\end{array}$ \\
\hline TW-5 & $12 / 8 / 92$ & Bennett pump & FS & $\begin{array}{l}\text { Water is clear } \\
\text { and odor free. }\end{array}$ \\
\hline
\end{tabular}


Table 4. RK1 sampling sumary for Decomber 1992 (contlnued)

\begin{tabular}{|c|c|c|c|c|}
\hline $\begin{array}{l}\text { Well } \\
\text { Name }\end{array}$ & $\begin{array}{l}\text { Date } \\
\text { sampled }\end{array}$ & $\begin{array}{l}\text { Sample } \\
\text { Mothod }\end{array}$ & $\begin{array}{l}\text { Parameter } \\
\text { suite }\end{array}$ & Comment: \\
\hline$T W-11$ & $12 / 7 / 92$ & Bennett pump & Fs & $\begin{array}{l}\text { Water is clear, } \\
\text { slight sulfur odor. }\end{array}$ \\
\hline$T W-12$ & $12 / 7 / 92$ & Bennett pump & FS & $\begin{array}{l}\text { Water is clear, } \\
\text { light sulfur odor. }\end{array}$ \\
\hline$T W-13$ & $12 / 7 / 92$ & Bennett pump & Fs & $\begin{array}{l}\text { Water is cloudy, } \\
\text { gray with light } \\
\text { sulfur odor. }\end{array}$ \\
\hline$T W-15$ & $12 / 3 / 92$ & Bonnett pump & FS & $\begin{array}{l}\text { Water is clear } \\
\text { and cor free. }\end{array}$ \\
\hline$T W-16$ & $12 / 4 / 92$ & Bonnett pump & Fs & $\begin{array}{l}\text { Water is ilear } \\
\text { and odor free. }\end{array}$ \\
\hline$T W-17$ & $12 / 4 / 92$ & Bennett pump & Fs & $\begin{array}{l}\text { Water is clear, } \\
\text { light oulfur odor. }\end{array}$ \\
\hline$T W-18$ & $12 / 7 / 92$ & Bennett pump & FS & $\begin{array}{l}\text { Wator is clear, } \\
\text { - light sulfur odor. }\end{array}$ \\
\hline VIW-1 & $12 / 9 / 92$ & Bennett pump & FS & $\begin{array}{l}\text { Water is clear, } \\
\text {-light sulfur } \\
\text { odor. }\end{array}$ \\
\hline CCW-1 & $12 / 9 / 92$ & Bennett pump & IS & $\begin{array}{l}\text { Water is olightly } \\
\text { cloudy, slight } \\
\text { sulfur odor. } \\
\text { well bore } \\
\text { obstructed at } \\
240 \mathrm{ft} \text {, pump } \\
\text { lowered to } 285 \mathrm{ft} \text {. }\end{array}$ \\
\hline
\end{tabular}




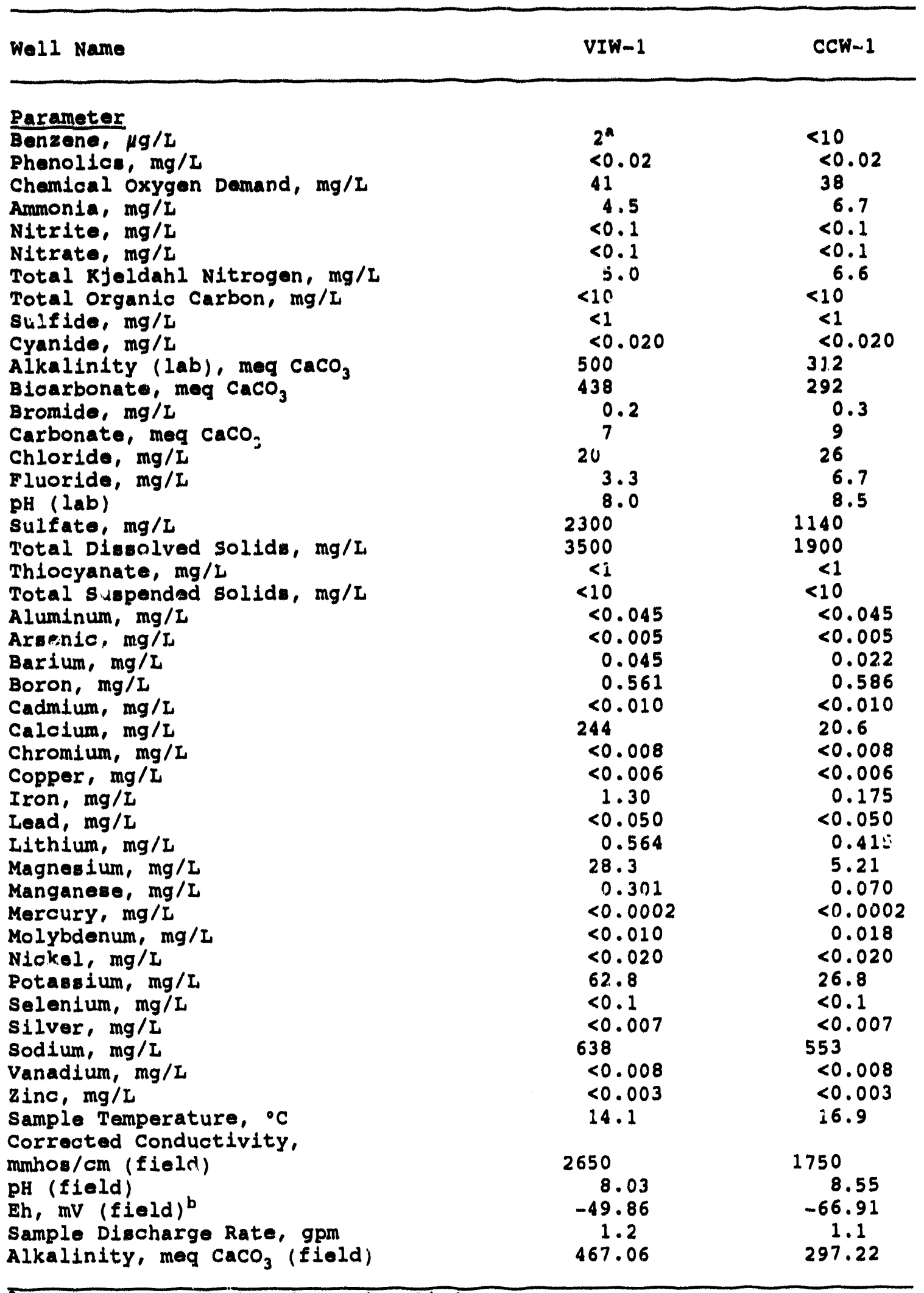

Concentration below detection limit

- corrected to standard $\mathrm{H}^{+}$electrode 
rable 6. Aneilyee for sanples Obtalned from coal soan Well.

\begin{tabular}{|c|c|c|c|c|}
\hline Well Name & EMW-1 & EMW-3 & $B M H-9$ & BMT-11a \\
\hline 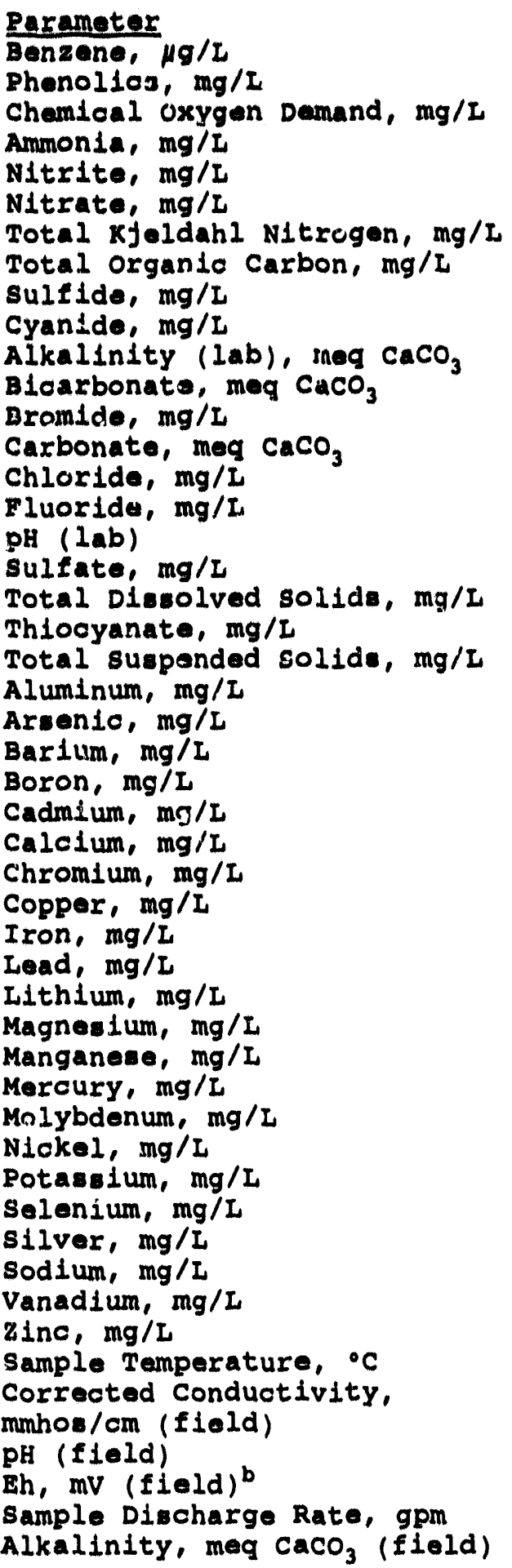 & $\begin{array}{c}13 \\
<0.02 \\
65 \\
3.5 \\
<0.1 \\
<0.1 \\
4.5 \\
17 \\
<1 \\
<0.02 \\
936 \\
917 \\
0.1 \\
19 \\
10 \\
1.5 \\
8.3 \\
590 \\
1890 \\
<1 \\
<10 \\
<0.045 \\
<0.005 \\
0.069 \\
0.023 \\
<0.010 \\
14.0 \\
<0.008 \\
<0.006 \\
0.066 \\
<0.050 \\
0.096 \\
7.05 \\
<0.003 \\
<0.0002 \\
<0.010 \\
<0.020 \\
6.48 \\
<0.1 \\
<0.007 \\
602 \\
<0.008 \\
<0.003 \\
9.0\end{array}$ & $\begin{array}{c}16 \\
<0.02 \\
93 \\
2.6 \\
<0.1 \\
<0.1 \\
3.3 \\
20 \\
<1 \\
<0.02 \\
785 \\
767 \\
<0.1 \\
18 \\
4.7 \\
1.2 \\
8.4 \\
540 \\
1600 \\
<1 \\
<10 \\
<0.045 \\
<0.005 \\
0.078 \\
0.023 \\
<0.010 \\
9.44 \\
<0.008 \\
<0.006 \\
0.106 \\
<0.050 \\
0.075 \\
5.33 \\
<0.003 \\
<0.0002 \\
<0.010 \\
<0.020 \\
<5.0 \\
<0.1 \\
<0.007 \\
528 \\
<0.008 \\
<0.003 \\
9.6 \\
<0\end{array}$ & 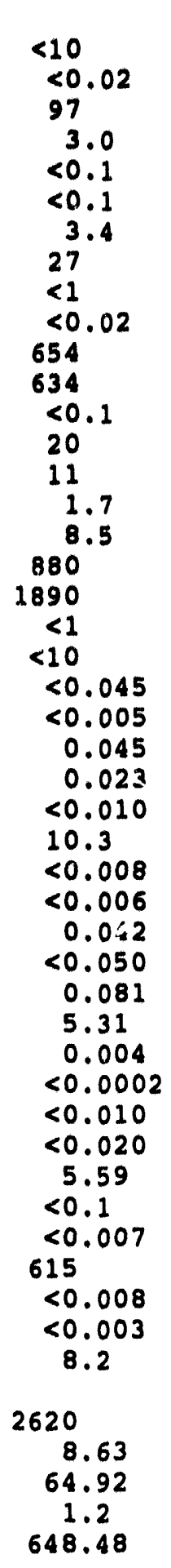 & 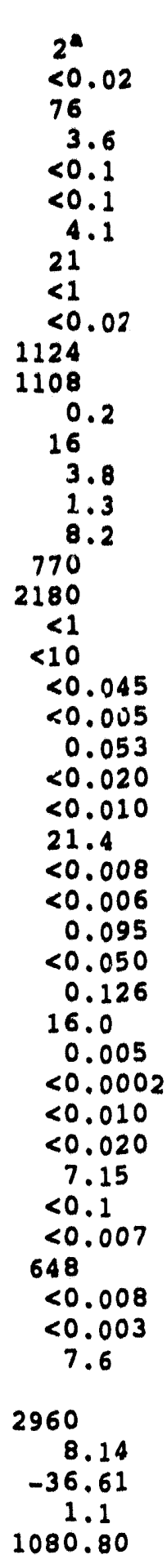 \\
\hline
\end{tabular}


Table 6. Analyses for samples Obtalned fron coal seam Welle (cont'd)

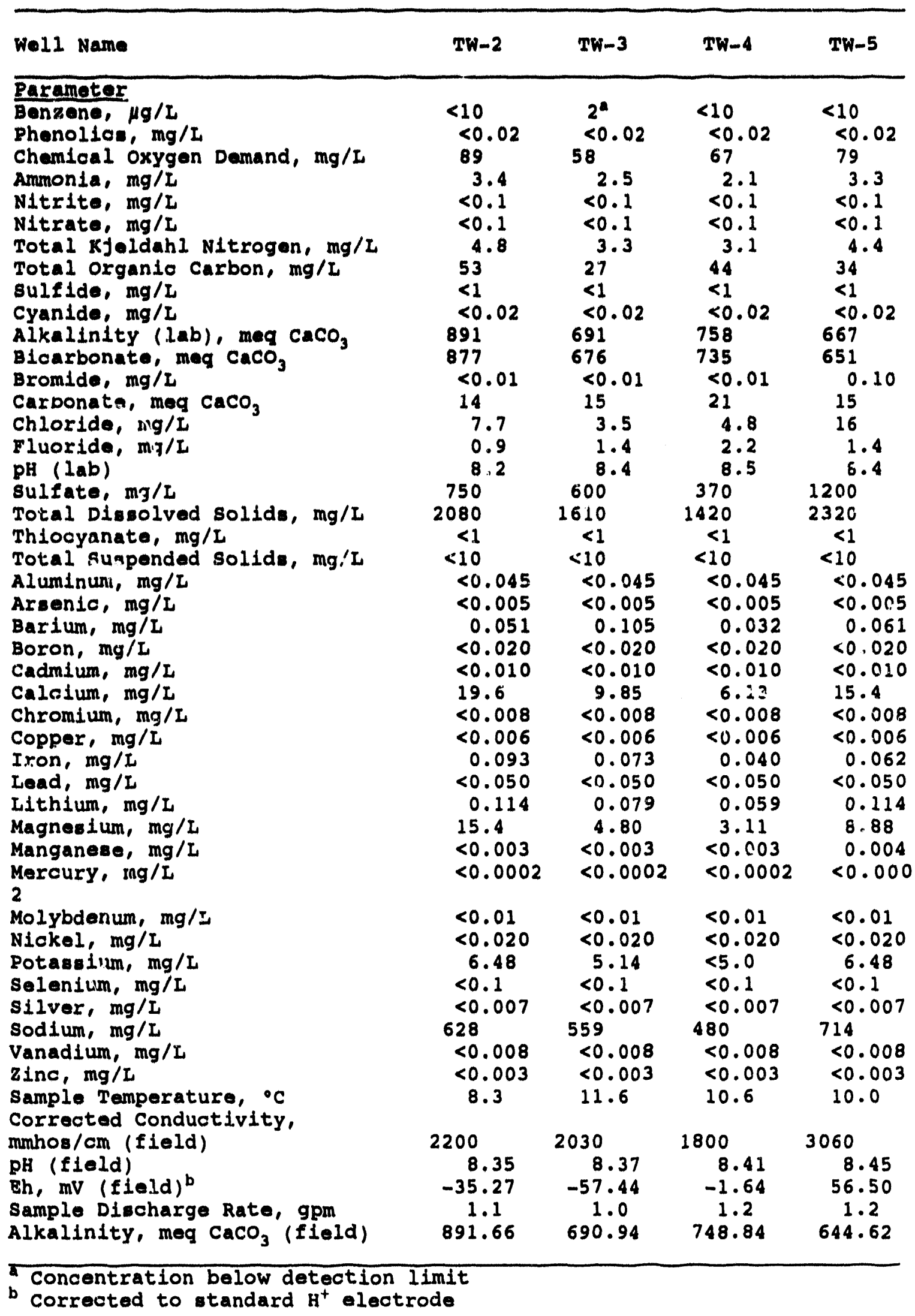


Table 6. Analyoe for sanples obtained from coal soan Wells (cont'd)

\begin{tabular}{|c|c|c|c|c|}
\hline Well Name & $T *-11$ & $T H-12$ & $T W-13$ & $T W-15$ \\
\hline 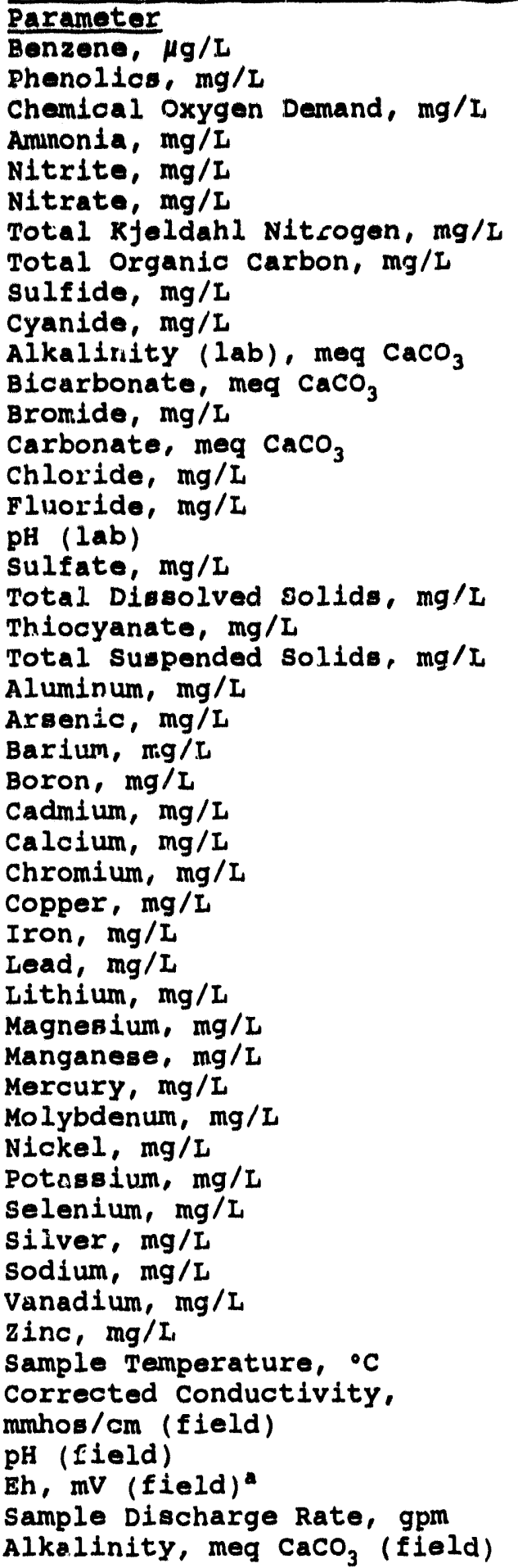 & $\begin{array}{c}<10 \\
<0.02 \\
77 \\
3.0 \\
<0.1 \\
<0.1 \\
3.9 \\
32 \\
<1 \\
<0.02 \\
810 \\
790 \\
<0.1 \\
19 \\
4.7 \\
1.3 \\
8.4 \\
470 \\
1550 \\
<1 \\
<10 \\
<0.045 \\
<0.005 \\
0.093 \\
<0.020 \\
<0.010 \\
20.5 \\
<0.008 \\
<0.006 \\
0.071 \\
<0.050 \\
0.096 \\
18.4 \\
0.004 \\
<0.0002 \\
<0.010 \\
<0.020 \\
6.03 \\
<0.1 \\
<0.007 \\
494 \\
<0.008 \\
<0.003 \\
8.0 \\
<0\end{array}$ & $\begin{array}{l}<10 \\
<0.02 \\
56 \\
2.8 \\
<0.1 \\
<0.1 \\
3.4 \\
15 \\
<1 \\
<0.02 \\
713 \\
698 \\
<0.1 \\
15 \\
4.0 \\
1.6 \\
8.4 \\
600 \\
1620 \\
<1 \\
<10 \\
<0.045 \\
<0.005 \\
0.056 \\
<0.020 \\
<0.010 \\
12.3 \\
<0.008 \\
<0.006 \\
0.057 \\
<0.050 \\
0.075 \\
9.05 \\
<0.003 \\
<0.0002 \\
<0.010 \\
<0.020 \\
<5.0 \\
<0.1 \\
<0.007 \\
540 \\
<0.008 \\
<0.003 \\
8.9\end{array}$ & $\begin{array}{c}<10 \\
<0.02 \\
75 \\
2.3 \\
<0.1 \\
<0.1 \\
3.0 \\
21 \\
<1 \\
<0.02 \\
672 \\
652 \\
<0.1 \\
20 \\
3.1 \\
1.9 \\
8.5 \\
420 \\
1460 \\
<1 \\
<10 \\
0.081 \\
<0.005 \\
0.127 \\
<0.020 \\
<0.010 \\
6.31 \\
<0.008 \\
<0.006 \\
0.090 \\
<0.050 \\
0.059 \\
3.41 \\
<0.003 \\
<0.0002 \\
<0.013 .90 \\
<0.020 \\
<5.0 \\
<0.1 \\
<0.007 \\
476 \\
<0.008 \\
<0.003 \\
8.9 \\
1910 \\
8.40 \\
<0 \\
<0 \\
<0 \\
<0 \\
<0\end{array}$ & $\begin{array}{c}<10 \\
<0.02 \\
69 \\
2.3 \\
<0.1 \\
<0.1 \\
3.3 \\
27 \\
<1 \\
<0.02 \\
740 \\
717 \\
<0.1 \\
23 \\
6.2 \\
2.7 \\
8.5 \\
420 \\
1460 \\
<1 \\
<10 \\
<0.045 \\
<0.005 \\
0.038 \\
<0.020 \\
<0.010 \\
6.24 \\
<0.008 \\
<0.006 \\
0.078 \\
<0.050 \\
0.061 \\
3.17 \\
<0.003 \\
<0.0002 \\
<0.010 \\
<0.020 \\
<5.0 \\
<0.1 \\
<0.007 \\
495 \\
<0.008 \\
<0.003 \\
6.9 \\
<0\end{array}$ \\
\hline
\end{tabular}

- Corrected to standard $\mathrm{H}^{+}$electrode 
Table 6. Analyses for samples Obtained from Coal sean Wells (cont'd)

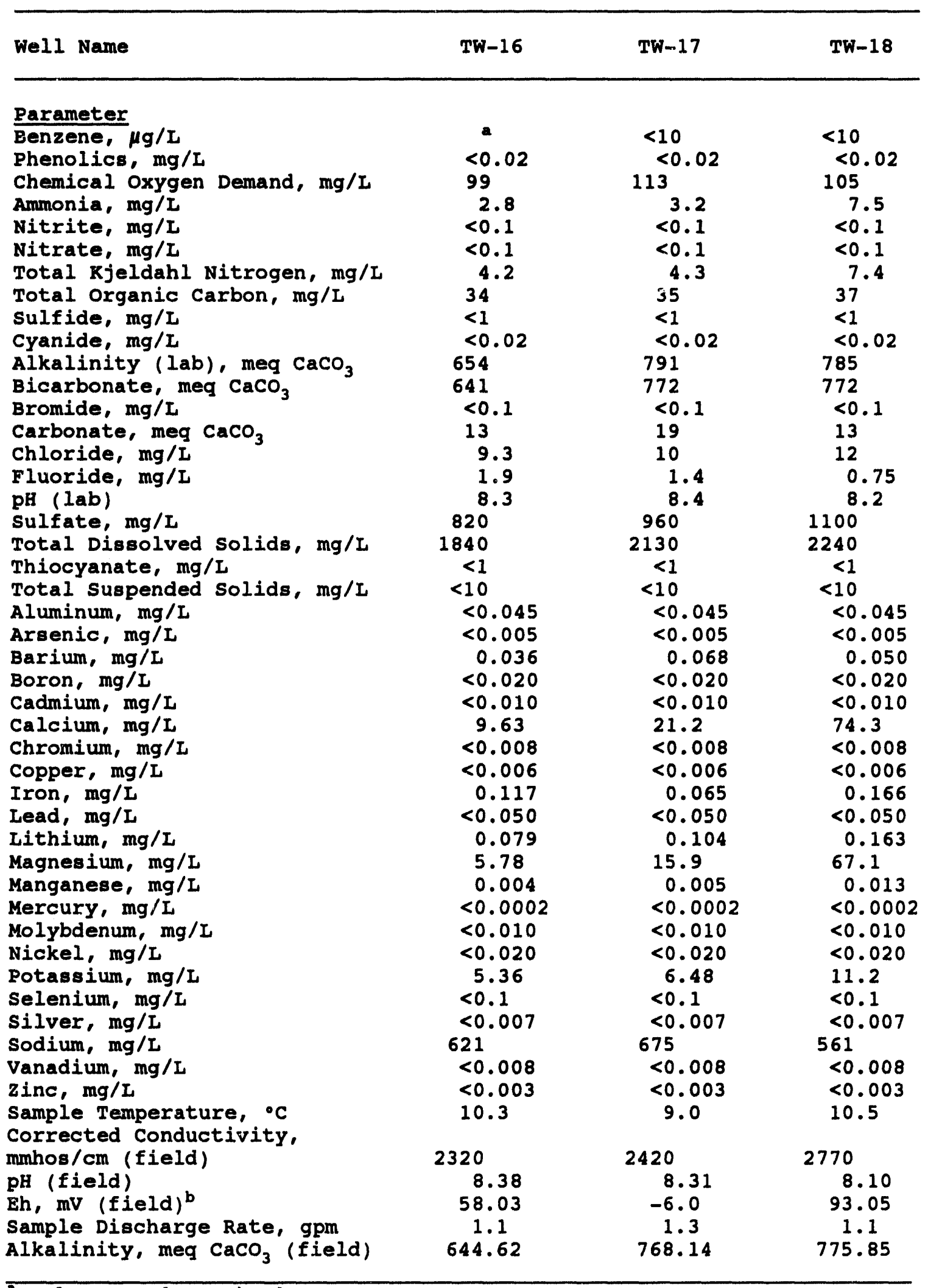

Value not determined

b corrected to standard $\mathrm{H}^{+}$electrode 
Table 7. Analyses for samples Obtained from Overburden Wells

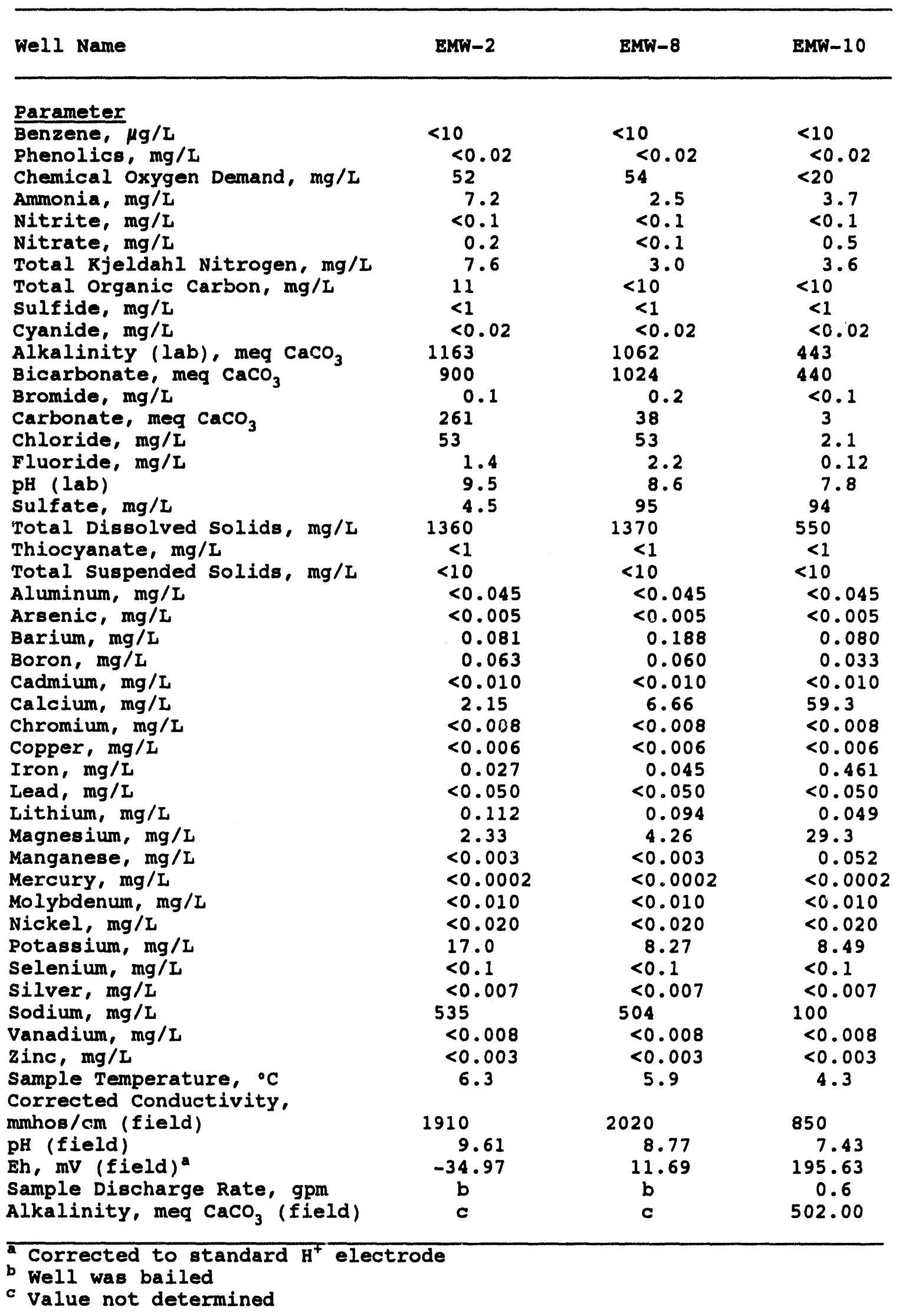


Table 8. Baseline Groundwater Parameters RK1 UCG Project, Hanna, Wyoming*

\begin{tabular}{lc}
\hline \multicolumn{1}{c}{ Parameters } & $\begin{array}{c}\text { Range of Concentrations } \\
\text { Raseline Coal Aquifer Wells, } \mathrm{mg} / \mathrm{L}^{\mathrm{b}}\end{array}$ \\
\hline TOC & $11-45$ \\
PNAs & Trace \\
Volatile organics & Trace \\
Phenols & $<0.020^{\mathrm{c}}$ \\
Ammonia & $2.4-7.9$ \\
Alkalinity (Field) & $638-855$ \\
pH & $7.7-9.24$ \\
Sulfates & $300-1400$ \\
TDS & $1360-2750$ \\
Cyanide & $<0.02$ \\
Boron & $<0.01-0.037$ \\
Aluminum & $<0.03-0.0253$ \\
Cadmium & $<0.01$ \\
Chromium & $<0.01$ \\
Copper & $<0.01$ \\
Lead & $<0.05$ \\
Mercury & $<0.00005$ \\
Nickel & $<0.01$ \\
Selenium & $<0.005$ \\
Arsenic & $<0.005$ \\
Barium & $0.016-0.162$ \\
Iron & $0.042-1.060$ \\
Manganese & $<0.005-0.024$ \\
Zinc & $<0.015$ \\
& \\
\hline & \\
\hline &
\end{tabular}

a From Covell et al. 1992

b The baseline analyses are for October and December 1986 (WRI 1986a and 1986b) and March and August 1987 (WRI 1987a and 1987b)

c Less than (<) indicates that the concentrations are below detection limits 
Table 9. Sumnary of Mothods and Accuracy, Precision, and Detection-Linit Objeatives

\begin{tabular}{|c|c|c|c|c|}
\hline Parameter & Method & $\begin{array}{c}\text { Accuracy, } \\
\text { - Blas }\end{array}$ & $\begin{array}{c}\text { Precision, } \\
\text { RSD }\end{array}$ & $\begin{array}{l}\text { Detection } \\
\text { Limits }\end{array}$ \\
\hline \multicolumn{5}{|l|}{ Anions } \\
\hline Alkalinity & EPA 310.1 & 25 & 5 & $10 \mathrm{mg} / \mathrm{L}$ \\
\hline $\mathrm{HCO}_{3}{ }^{-}, \mathrm{CO}_{3}{ }^{2-}$ & SM $406 C$ & $\mathrm{NA}^{\mathrm{b}}$ & $\mathrm{NA}^{\mathrm{b}}$ & $\mathrm{NA}^{\mathrm{b}}$ \\
\hline $\mathrm{Br}^{-}, \mathrm{Cl}^{-}$ & EPP. 300.0 & NA & NA & $0 . / \mathrm{mg} / \mathrm{L}$ \\
\hline $\mathbf{F}^{-}$ & EPA 340.2 & 5 & 5 & $0.2 \mathrm{mg} / \mathrm{L}$ \\
\hline & SM 429, modified & & & \\
\hline$s^{2-}$ & EPA 376.1 & $N A^{b}$ & $\mathbf{N A}^{b}$ & $1 \mathrm{mg} / \mathrm{L}$ \\
\hline $\mathrm{SO}_{4}^{2-}$ & EPA 300.0 & NA & NA & $0.1 \mathrm{mg} / \mathrm{L}$ \\
\hline
\end{tabular}

Cyanides

$\mathrm{CN}^{-}$

$\mathrm{SCN}^{-}$

Nitrogen

Free $\mathrm{NH}_{3}-\mathrm{N}$

Total KJeldahl $\mathrm{NH}_{3}-\mathrm{N}$

$\mathrm{NO}_{2} / \mathrm{NO}_{3}-\mathrm{N}$

EPA 300.0

EPA 420.2

CLP modified

TCLP modified

\section{Organics}

Phenol

Volatiles

Semivolatiles"
NA

40

15

5

10

40
10

NA

$0.10 \mathrm{mg} / \mathrm{L}$

10

$0.5 \mathrm{mg} / \mathrm{L}$

$0.2 \mathrm{mg} / \mathrm{I}$

A.

10

25

Sample and analyte

dependent

25

Sample and analyte dependent, typically

$10 \mu \mathrm{g} / \mathrm{L}$

Other

COD

Conductivity

Eh

pH

Temperature

TOC

EPA 410.4
EPA 120.1
USGS $(1976)$
EPA 150.2
SM 212
SM 505

(combustion, coulometric

titration)

$\begin{array}{lll}N A^{b} & N A^{b} & 50 \mathrm{mg} / L \\ 5 & 10 & \\ N A^{b} & N A^{b} & \\ 1 & 5 & \\ N A^{b} & N A^{b} & 10 \mathrm{mg} / L \\ 15 & 15 & \end{array}$


Table 9. sumary of Motbods and Aocuracy, Preolelon, and Dotection-Lintt objectives (continued)

\begin{tabular}{|c|c|c|c|c|}
\hline Parameter & Method & $\begin{array}{c}\text { Accuracy, } \\
\text { \& Bias }\end{array}$ & $\begin{array}{c}\text { Precision, } \\
\text { RSD }\end{array}$ & $\begin{array}{l}\text { Detection } \\
\text { Limits }\end{array}$ \\
\hline TDS & EPA 160.1 & $N A^{b}$ & $N A^{b}$ & $10 \mathrm{mg} / \mathrm{L}$ \\
\hline TSS & EPA 160.2 & $N A^{b}$ & $N A^{b}$ & $4 \mathrm{mg} / \mathrm{I}$ \\
\hline
\end{tabular}

Trace Elements

\begin{tabular}{|c|c|c|c|c|c|}
\hline Aв & EPA 260.2 & 20 & 20 & 5 & $\mu \mathrm{g} / \mathrm{L}$ \\
\hline Se & EPA 270.2 & 20 & 20 & 5 & $\mu g / L$ \\
\hline $\mathrm{Hg}$ & EPA 245.1 & 20 & 20 & 0.5 & $\mu \mathrm{g} / \mathrm{I}$ \\
\hline $\begin{array}{l}\text { B, Ba, Ca, Cd, Cr, } \\
C u, F e, L 1, M o, N 1 \text {, } \\
\text { V, Zn }\end{array}$ & EPA 200.7 & 20 & 20 & 0.01 & $\mathrm{mg} / \mathrm{L}$ \\
\hline Al & EPA 200.7 & 20 & 20 & 0.03 & $\mathrm{mg} / \mathrm{L}$ \\
\hline $\mathbf{K}$ & EPA 200.7 & 20 & 20 & 5 & $\mathrm{mg} / \mathrm{L}$ \\
\hline$M g, M n$ & EPA 200.7 & 20 & 20 & 0.005 & $\mathrm{mg} / \mathrm{L}$ \\
\hline $\mathrm{Na}$ & EPA 200.7 & 20 & 20 & 0.5 & $\mathrm{mg} / \mathrm{L}$ \\
\hline $\mathrm{Pb}$ & EPA 200.7 & 20 & 20 & 0.05 & $\mathrm{mg} / \mathrm{L}$ \\
\hline
\end{tabular}

- RSD - Relative standard Deviation (or coefficient of variation) $=\mathbf{s} / \mathrm{m} \mathbf{x} 100$, where $\mathrm{g}$ is the standard deviation and $\mathrm{m}$ is the mean

b NA = Not available

c Includes acid extractables, base/neutral extractables, heterocyclics, and PNAs

References: EPA analyses (U.S. EPA 1983), SM analyses (AWWA 1985), CLP methods (U.S. EPA 1985), USGS method (Wood 1976) 
Table 10. Wells Exceeding Bighest Baneline Concentrations of Indicator Constituents, December 1992, mg/L

\begin{tabular}{ll}
\hline Coal Seam Wells & Overburden Wells \\
\hline$\underline{T D S}$ & $\frac{\text { TDS }}{\text { EMW-2 }=1360}$ \\
VIW-1 = 3500 & EMW-8 $=1370$ \\
HBC $=2750$ & HBC $=1040$ \\
TOC & TOC \\
TW-2 $=53$ & EMW-2 $=11$ \\
HBC $=45$ & HBC $=10$ \\
BOron & Ammonia \\
VIW-1 $=0.561$ & EMW-2 $=7.2$ \\
CCW-1 $=0.586$ & HBC $=4.7$ \\
HBC $=0.037$ & \\
\hline
\end{tabular}

Baseline concentrations listed in Table 8 . 
Table 11. Analyses for Quality Aseurance/Quality Control samples

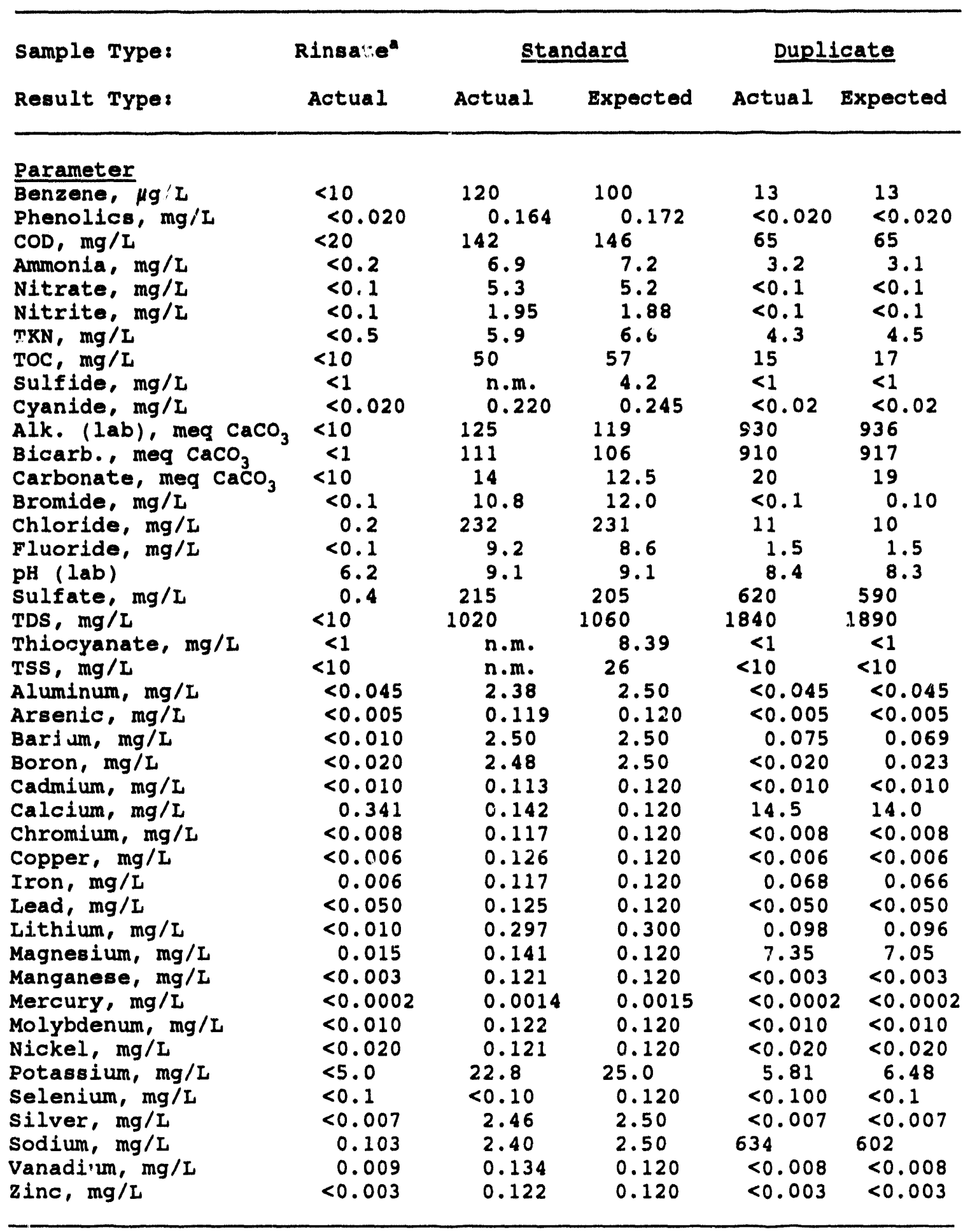

a Expected results for rinsate sample parameters are below detection limit. 
American Water Work Asociation, 1985, standard Methods for the Examination of Water and Wastewater, 16th Bdition, Washington, DC.

Covell, J.R., S.R. Iindblom, D.S. Dennis, and J.E. Boysen, 1992, Groundwater Restoration at the Rocky Mountain 1 Underground Coal Gasification site, Hanna, Wyoming. WRI Topical Report to GRI, Laramie, WY, WRI-91-R039, GRI-91/0181.

U.S. Environmental Protection Agency, 1983 , Method for Chemical Analyses of water and Waste. Washington, DC, EPA-600/4-79-020.

U.S. Environmental Protection Agency, 1985, Contract Laboratory Program statement of Work for Organics, Multi-Media, Multi-Concentration. Washington, DC.

Western Research Institute, 1986a, Baseline Sampling for the RMI site, Hanna, Wyoming, October 1986. Unpublished WRI Sampling and Analyses Report to Stearns Catalytic corporation, Laramie, WY, WRI-91-R047.

Western Research Institute, $1986 \mathrm{~b}$, Baseline sampling for the RMl site, Hanna, WY, December 1986. Unpublished WRI Sampling and Analyses Report to Stearns Catalytic Corporation, Laramie, WY, WRI-91-R048.

Western Research Institute, 1987a, Baseline Sampling for the RMI site, Hanna, WY, March 1987. Unpublished WRI Sampling and Analyses Report to Stearns Catalytic Corporation, Laramie, WY, WRI-91-R049.

Western Research Institute, 1987b, Baseline sampling for the RMI site, Hanna, WY, August 1987. Unpublished WRI Sampling and Analyses Report to Stearns Catalytic Corporation, Laramie, WY, WRI-92-R003.

Wood, w.W., 1976, Guideline for collection and Field Analyses of Groundwater samples for selected Unstable constituents, in Techniques of Water-Resources Investigations of the United States Geological survey, Chapter D2. U.S. Government Printing office, Washington, DC. 
APPIATIX 8

vod Analytical Results 
IA

VOLATILE ORGANICS ANALYSIS DATA SHEET

Lab NameiWestarn Reseerch Inststut Contracti68-02-0017

EPA SAMPLL: NO.

Lab COdOIWRI

Motrixi (soll/water) WATER

Sample wt/roll $5.00(\mathrm{~g} / \mathrm{ml}) \mathrm{ML}$

Levals (low/med) LOW

$x$ Masetures not dace.

GC ColumniDQ-624

$1080.53(\mathrm{~mm})$

Sold Extract Volumaio

(uL)

SAS Na.

SOG NO.:RM-1

Lab Samp!e 10:

Leb F11. 10, UB268

Dete Recelveds 11

Dete Analyzod 1/2/14/92

Dilution Factori $\quad 1.0$

Soll Aliquot Volumeio

(uL)

CONCENTRATION UNITS:

CAS NO.

COMPOUNO

(WO/L or UQ/KO) UG/L $Q$

\begin{tabular}{|c|c|}
\hline $74-67-3-\cdots \cdots-\cdots$ - chlor & 10,12 \\
\hline $74-83-9-----m-9-8$-8romomethene & 10.14 \\
\hline $75-91-1-\cdots-\cdots-\cdots-4$ ln $x /$ chloride & 10.14 \\
\hline $75-90-3-\cdots-----$ chloreethere & 10.14 \\
\hline $75-89-2-\ldots \ldots-\cdots$ Methylene chlocide & 10,14 \\
\hline $67-64-1-\cdots-\cdots---$ Acelone & $10+14$ \\
\hline $75-15-0-\cdots-------c$ crbon 018ulfude & 19.14 \\
\hline $75-35-1-\cdots-----1 \mid 1-0$ denlorenthene & 10.18 \\
\hline 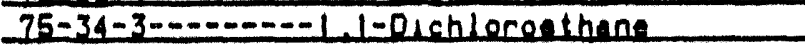 & in.15 \\
\hline 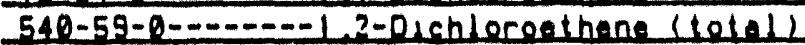 & 10.14 \\
\hline $67-66-3 \ldots-\cdots---$ Chloroform & 10,14 \\
\hline $107-06-2-\cdots--1 / 2-0$ 1ahlerenthene & 10.14 \\
\hline $78-93-3-\cdots-\cdots---2-8$ utenone & 10.12 \\
\hline $71-55-6-\ldots-\cdots--1, J$ J-Irjehleresethene & 10.14 \\
\hline $56-23-5 \ldots-\cdots-\cdots-$ Grobon Ietrechloride & 10,14 \\
\hline $75-27-4-\cdots-\cdots--B$ remodiahloromethane & 10,14 \\
\hline $78-87-5-\cdots---\cdots-12-0$ lahlecooronene & $10,1 \overline{1}$ \\
\hline 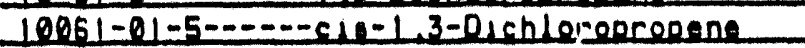 & 10.14 \\
\hline $79-91-6-\ldots-\cdots---$-Irichloroethene & 10.14 \\
\hline $121-48-1-\cdots-\cdots-0$ bromochloromethane & 10.12 \\
\hline $79-00-5-\cdots-----1 /$ / 2-Iriahleroethene & 10,14 \\
\hline $71-43-2-\cdots-\cdots---$ Benzene $^{2}$ & 10.14 \\
\hline $10061-02-6-\cdots---$ trans -1 3-Duchloroorogene & 10,14 \\
\hline $75-35-2-\cdots-\cdots-$ - Bromoform & $10.1 \overline{2}$ \\
\hline $108-10-1-\cdots \cdots-1 \cdot$ Methy1 $-2-P$ & 10.14 \\
\hline $591-78-6-\cdots-\cdots--2-$ Hexanene & 10.14 \\
\hline $127-18-1-\ldots-\ldots-$-Intrechlerenthene & 10.14 \\
\hline $79-34-5---\cdots---1,1,2$ 2-Intreahleresthene & 10.12 \\
\hline 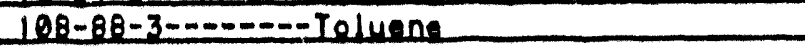 & 10,14 \\
\hline $108-90-7-\ldots-\ldots-$-Chlorobenzene & 10.14 \\
\hline$|a 9-4|-1-\ldots-\ldots--$ Ethylbenzene & 10,12 \\
\hline $100-4^{2}-5-\cdots----5$ syrene & 10.14 \\
\hline $1330-20-7 \cdots-\cdots-x$ ylene (10tal) & 10,14 \\
\hline
\end{tabular}


in

VOLATILE ORGANICS ANALYSIS DATA CHEET

Lab Nameiwastern Research Inststut Contracti68-02-0017 I EMWI

Lab CodolWRI Case No.110201 SAS No.1 SDG No.tRM-1

Matrixi (1011/wator) WATER

Sample wetroll 5.00 (oiml) ML

Lovell (low/mod) LOW

* Mossturat not dac.

6C Columnido-624

$1010.53(\mathrm{~mm})$

Soll Extract Volumeio

(ul )

COMPOUND

CAS NO.
Lab Sample ID:

Lab File ID: RMIIO

De' I Recelved: $12 / 10 / 92$

Date Analyzod:12/15/92

Didution Factori $\quad 1.0$

Soll Allquot Volumeto

(uL)

CONCENTRATION UNITS:

(UO/L or UO/KO) UG/L O

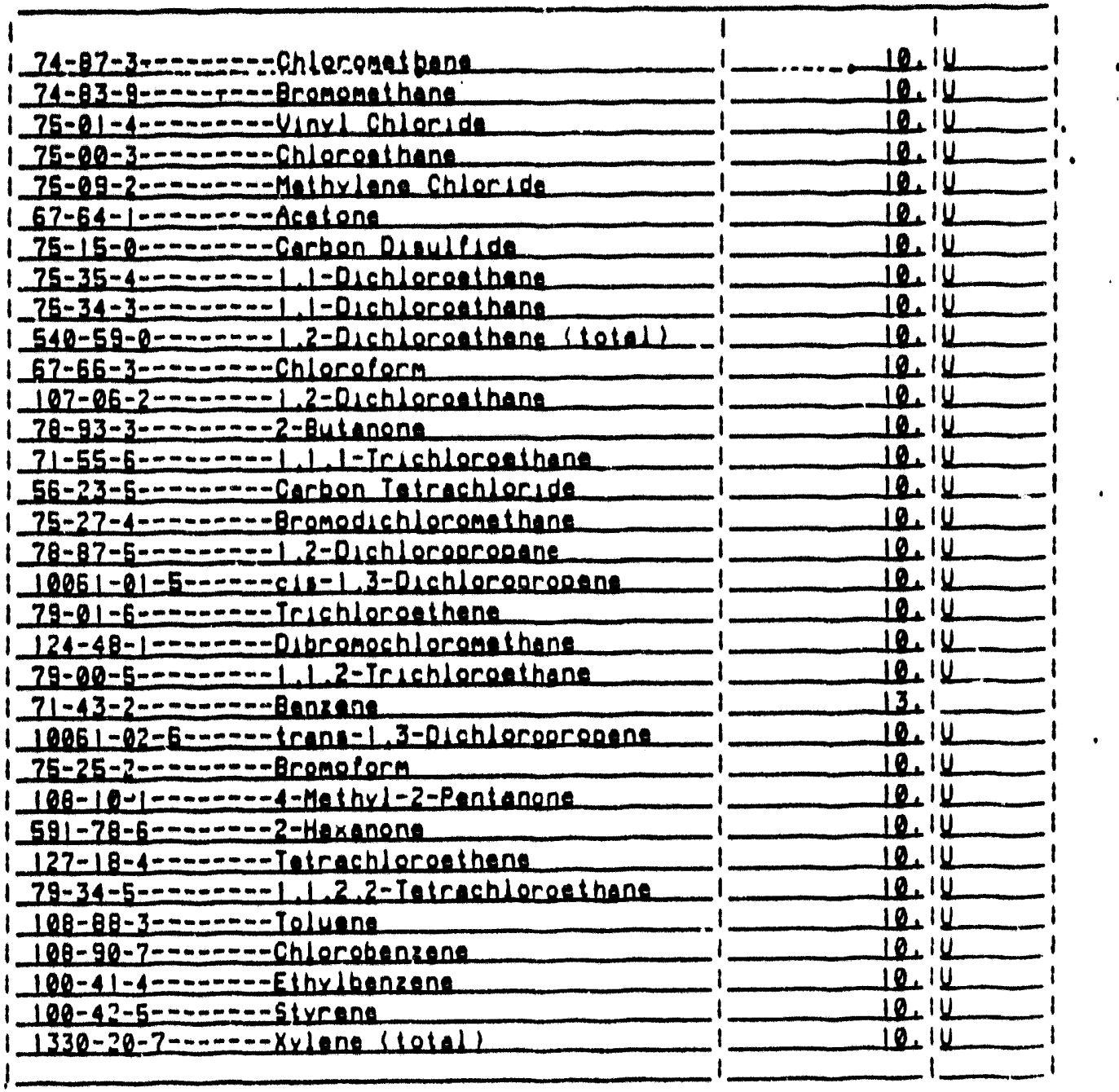


IA

VOLATILE ORGANICS ANALYSIS DATA SHEET

EPA SAMPLE NO.

Lab Nameitestern Research Instifut Contracti68-02-0017

1 EMW?

Lab CodoIWRI COAE NO.110201 SAS NO.1 SOG No. IRM-I

Matrixl (soll/watar) WATER

Lab Sample IDI

Sample wt/rolt $\quad 5.00(0 / m) \mathrm{ML}$

Lab Fil. 101 RMIIS

Levals (low/med) LOW

Date Receiredi12/10/82

X Maseturet not dec.

Date Analyzodi12/15/92

GC Columning-624

$1010.53(\mathrm{~mm})$

Dilution Fector: $\quad 1.0$

Soll Entract Volumato

(uL)

Soll Aliquot Volumeio

(uL)

CAS ND.

COMPOUND

CONCENTRATION UNITSI

(Ug/L or UQ/KO) UG/L Q

\begin{tabular}{|c|c|}
\hline $24-87-3 \cdots \cdots-\cdots-$ Chloromathing & 10. 14 \\
\hline $24-83-9-\cdots-\cdots--8$ remoget har 2 & 19,14 \\
\hline $75-01-1 \cdots \cdots-\cdots-1 / n y l$ Chlorde & 10.14 \\
\hline $75-90-3-\cdots \cdots---$ Chlorogthane & 10,14 \\
\hline 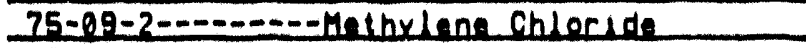 & 2,1 \\
\hline $62-54-1 \cdots \cdots \cdots--1$ & 10.14 \\
\hline $75-15-9 \cdots-\cdots-\cdots-$ Crebon Disulelde & 10.14 \\
\hline $75-35-4 \cdots \cdots-\cdots-1,1-0$ uahlorgethens & 10.14 \\
\hline $25-34-3 \cdots \cdots-\cdots-1,1-0$ uahloroethene & 10.12 \\
\hline $540-59-0-\cdots \cdots-1,2-0$ Lachlore & 10,11 \\
\hline $67-65-3-\cdots-\cdots-\cdots-$ chlorolorm & 19,12 \\
\hline $107-05-2-\cdots-\cdots-1,2-0$ iahleroethene & 10,14 \\
\hline $38-93-3-\cdots-----2-$ Butenone & 10,14 \\
\hline $71-55-6 \cdots \cdots \cdots-1,1,1-$ Ir lahloroethane & 10.14 \\
\hline $56-23-5-\cdots---\cdots-$ Chebon in & 10.14 \\
\hline $25-27-1-\cdots \cdots--$ - Bromoduch & 10.11 \\
\hline $78-87-5-\cdots-\cdots-1,2-0$ lechlorooroonne & 10.11 \\
\hline $10051-01-5------011-1,3-019 h$ loroorogene & 10,14 \\
\hline 29-01-6-------- Iruahloreathene & $19+14$ \\
\hline $124-18-1 \cdots \cdots-\cdots-D$ Dipremechlor & 10.14 \\
\hline 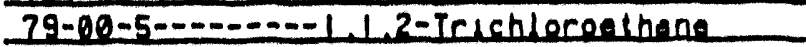 & 10,11 \\
\hline $21-43-2-\cdots-\cdots-\cdots$ - Bnnenge & 10.14 \\
\hline $10051-02-6-\cdots---1$ rens $-1,3-0$ & 19,14 \\
\hline $75-35-2-\cdots-\cdots--8$ romolocm & 19.14 \\
\hline $108-10-1-\cdots \cdots-\cdots-$ Methy $1-2-$ Pentanone & 19.12 \\
\hline $591-78-6 \cdots \cdots-\cdots-2-$ Hexnnone & 10,14 \\
\hline $127-18-1-\cdots-\cdots-$ Intenahleronthene & $19+14$ \\
\hline $74-34-5 \cdots-\cdots-\cdots-1,2,2-$ Intreahlocoethene & 10.11 \\
\hline $108-88-3-\cdots-\cdots-10$ luene & 10.14 \\
\hline $198-99-7-\cdots-\cdots-c h$ lorobenzene & 10.11 \\
\hline $199-11-1-------5$ - Shy loenzene & 10,12 \\
\hline $190-42-5-\cdots-\cdots-$ Styreng & 10.11 \\
\hline $1330-20-7 \cdots-\cdots-x \times$ lene (totell & 10.12 \\
\hline
\end{tabular}


IA

VOLATILE ORGANICS ANALYSIS DATA SHEET .

Lab Namaiwestern Research Instifut

Lab CodeIWRI

Case No.110201

SAS No.1

Lab Samplo 10 :

Somple wt/volt $5.00(\mathrm{~g} / \mathrm{ml}) \mathrm{ML}$

Levels (low/mod) LOW

X Mossturas not dec.

6C ColumniDB-624

$1010.53(\mathrm{~mm})$

Sold Extract Volumeto

(uL)

COMPOUND

CAS NO. CONCENTRATION UNITS:
EPA SAMPLE NO.

$$
1
$$

1 EMW3

017

SDG NO. RRM-1
Dete Receivedi/2/10/92

Date Analyzadi/2/15/92

Dilution Factor: 1.0

Soll Aliquot Volumeio

(uL) (UO/L or Ug/Kg) UG/L Q

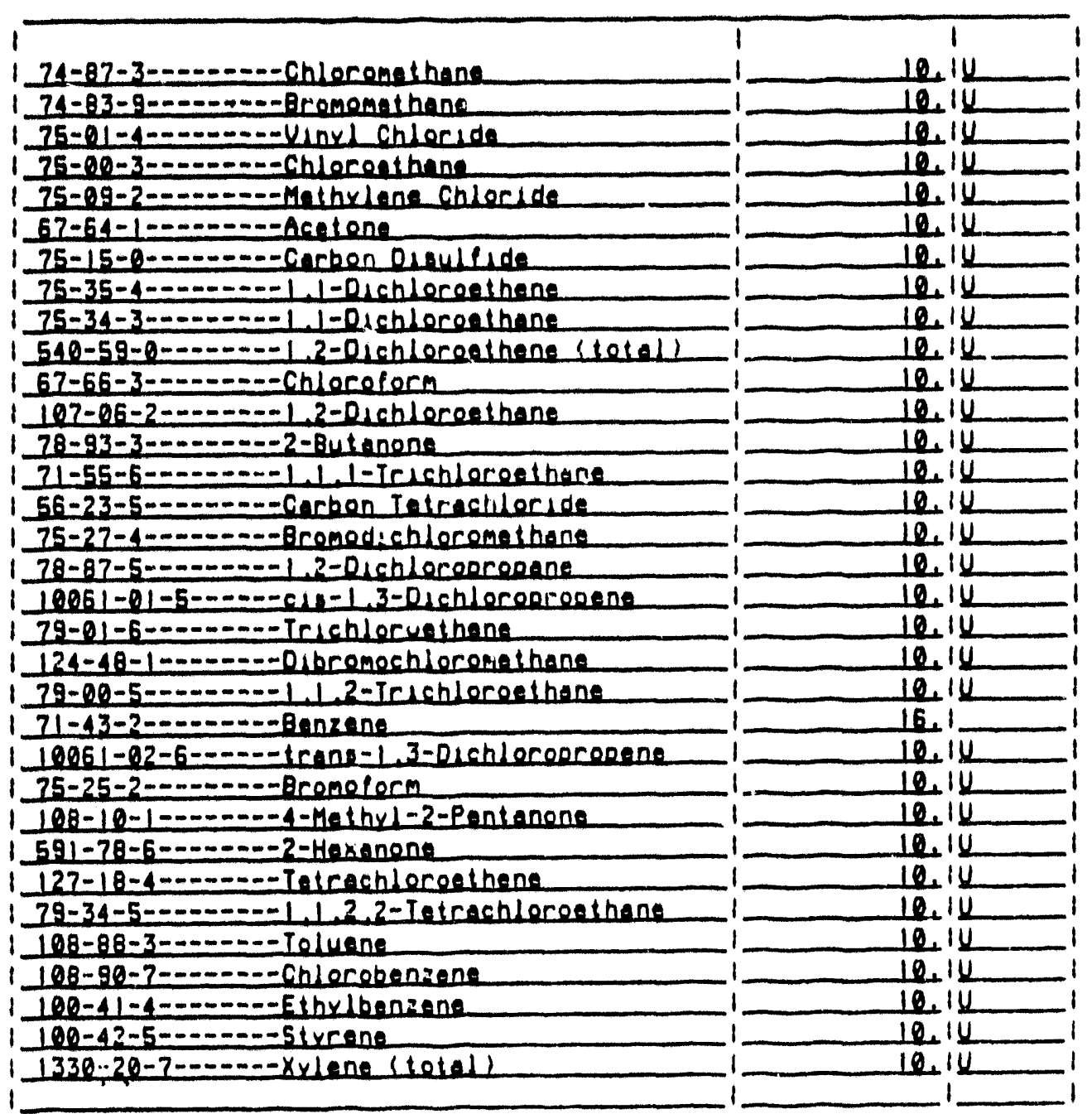


IA

VOLATILE ORGANICS ANALYSIS DATA SHEET

EPA SAMPLE NO.

Lab Nameiwestern Research Instilut Contracti68-02-0017

EMW3-DUP

Lab CodeiWRI

Case No. 110201

SAS NO.:

SDG NO. IRM-1

Matrixi (sold/watar) WATER

Sample wt/voll $5.00(\mathrm{~g} / \mathrm{ml}) \mathrm{ML}$

Leveli (low/med) LOW

X Holsturar not dac.

OC ColumniDB-1324

ID: $0.53(\mathrm{~mm})$

Sold Extract Wolumeio

(uL)

(UL)

CONCENTRATION UNITS:

CAS NO.

COMPOUND
Sold Aliquot Volume:o

Lab Sample I0I

Lab Fil IDI RMI28

Date Recelvedi/2/10/92

Date Analyzedi12/17/92

Dilution Factor: $\quad 1.0$

(Ug/L or Ug/Kg) UG/L Q

\begin{tabular}{|c|c|}
\hline $74-87-3-\cdots \cdots-\cdots-$ Chloromethane & 10. 1 \\
\hline $74-83-9-\cdots \cdots-\cdots-8$ romomethane & 10.12 \\
\hline $75-01-4 \cdots-\cdots-\cdots-v_{\text {iny }}$ Chlocide & 10.11 \\
\hline $75-99-3-\cdots--\cdots-$-Chloreethane & 10.11 \\
\hline $75-09-2=-\cdots-\cdots-M e t h y d e n e$ chloride & 10.11 \\
\hline $67-54-1-\cdots \cdots \cdots-\cdots-A c 2$ lone & 10.14 \\
\hline $75-15-\theta-\cdots \cdots-\cdots-\operatorname{carbon}$ Disulfude & 10.12 \\
\hline $75-35-4-\cdots-\cdots--1,1-0$ uchloreethene & 10.11 \\
\hline $25-34-3-\cdots-\cdots-1,1-D i c h l o r e e t h a n e$ & 10.11 \\
\hline $540-59-\theta-\cdots-\cdots-12-0$ ichloregthene (total) & 10.11 \\
\hline $67-56-3-\cdots \cdots-\cdots$ chloreform & 10.11 \\
\hline $107-06-2-\cdots-\cdots-1,2-0$ ichleroethane & 10.11 \\
\hline $78-93-3-=-\cdots-\cdots-2-8$ utenone & 10,12 \\
\hline $71-55-6 \cdots-\cdots-\cdots-1,1-$ Ir ichlereathane & 10,14 \\
\hline $56-23-5-=-\cdots---$ Cerbon Ietrachloride & 10,15 \\
\hline $25-27-4--=-\cdots-$ Bromedichloremethane & 10.12 \\
\hline $78-87-5-\cdots-\cdots---1,2-0$ \& a hloreorooene & 10,14 \\
\hline $10051-01-5 \cdots-\cdots-c 18-1,3-0$, chlor oorooene & 10,14 \\
\hline 79-01-6-- - - - - - Ir sahloroethene & $10+1 \overline{1}$ \\
\hline $124-48-1=\cdots-\cdots-D$ Dibromochloromethane & 10.11 \\
\hline 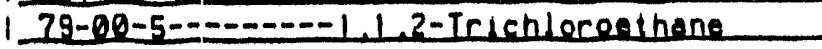 & 10,14 \\
\hline $71-13-2-\cdots-\cdots-\cdots-$ Benzene & $13+1$ \\
\hline $10061-02-6-\cdots-\ldots-t c a n s-1$ 3-DLehloreorooene & 10.11 \\
\hline $75-25-2 \cdots \cdots-\cdots-$ Bromoform & 10,14 \\
\hline $108-10-1-\cdots-\cdots-1-$ Methel-? - Pentenene & $10,1 \bar{L}$ \\
\hline $591-28-6=-\cdots-\cdots-2-$ Hexanene & 10,14 \\
\hline $127-18-4=---\cdots-$ - Ietrechloroethene & 10.11 \\
\hline $29-34-5-\cdots-\cdots-\cdots-1,2,2-I$ - trechloroethene & 10.11 \\
\hline $108-88-3-\cdots-\cdots--I_{0}$ luene & $19+11$ \\
\hline $108-99-7=--\cdots--$ Chlorobenzene & 10,11 \\
\hline$|9 P-4|-4=-\cdots---$ Elhylbenzene & 10.11 \\
\hline $100-42-5-\cdots-\cdots-5$ tyrene & 10.11 \\
\hline $1330-30-7 \cdots \cdots-\cdots \times-1$ ene (10tal) & 10.11 \\
\hline
\end{tabular}


IA

VOLATILE ORGANICS ANALYSIS DATA SHEET
Case No.:10201

SAS No. 1
EPA SAMPLE NO.

1

1 EMWB

Lab Code:WRI

Matrix: (soil/water) WATER

SDG No.:RM-1

Lab Sample ID:

Sample wt/vol: $5.00(0 / m) \mathrm{ML}$

Lab File IO: RMIIO

Level: (low/med) LOW

Date Received:12/10/92

X Molstunes not dec.

Dete Analyzed:12/14/92

6C Column:DB-624

IO: $0.53(\mathrm{~mm})$

Dilution Factor: $\quad 1.0$

Sold Extract Volumeio

(uL)

Soll Aliquot Volume:0

(uL) .

CAS NO.

COMPOUND

CONCENTRATION UNITS:

(Ug/L or Ug/Kg) UG/L Q

\begin{tabular}{|c|c|}
\hline $74-87-3 \cdots \cdots-\cdots-$ Chlor omethene & 10.11 \\
\hline $74-83-9-\cdots-\cdots--8$ comomethene & 10.11 \\
\hline $75-01-4-\cdots-\cdots--v_{\text {inyl }}$ Chloride & 10,14 \\
\hline $35-00-3-\cdots-\cdots---$ chloroethane & 10,11 \\
\hline 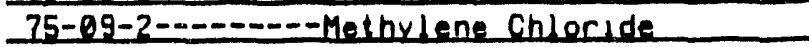 & 10,11 \\
\hline $67-64-1-\cdots-\cdots--$ Acetene & $10,1 \ldots$ \\
\hline $75-15-9-\cdots-\cdots--$ Cerbon Disulfide & 10.11 \\
\hline $75-35-4-\cdots-\cdots--1$, -Dichlocesthene & 10,112 \\
\hline $75-34-3-\cdots-\cdots--1,1-$ Dichlor oe thane & 10,14 \\
\hline $54 \theta-59-\theta-\cdots-\cdots-1,2-0$ ichlorest hene (total) & 10.14 \\
\hline $67-66-3-\cdots-\cdots---$ Chlor oform & 10,14 \\
\hline $107-06-2-\cdots \cdots--12^{2}-0$ ichloroethane & 10,14 \\
\hline $78-93-3-\cdots-\cdots---2-$ Butenone & 19.14 \\
\hline $21-55-6-\cdots-\cdots--1,1,1-$ Irichloroethane & 10,14 \\
\hline $56-23-5 \cdots-\cdots--$ Cerbon Tetrachloride & 10,14 \\
\hline $75-27-4-\cdots-\cdots--$ - Bromodichloromethane & 10,14 \\
\hline $78-87-5 \cdots-\cdots---1,2-0$ ichlorepropene & 18.11 \\
\hline $10061-01-5 \cdots-\cdots-018-1,3-0$ 1ahloreorooene & 10.14 \\
\hline 79-91-6--- - - - - Irichloroethene & 10,112 \\
\hline $124-48-1-\cdots-\cdots-0$ ibromochloromethane & 10,11 \\
\hline $79-90-5-\cdots-\cdots-1,1,2-$ Irichloreethane & 10.14 \\
\hline $71-43-2-\cdots-\cdots--$ Benrene & 10,14 \\
\hline 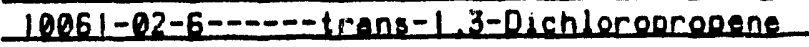 & 10.iL \\
\hline $75-25-2----\cdots---8$ romoform & 10,11 \\
\hline $108-10-1-\cdots-\cdots-1-$ Methy 1-2-Pentenone & 10.11 \\
\hline $591-78-6-\cdots-\cdots-2-$ Hexenone & $10_{1}: 1$ \\
\hline $127-18-4-\cdots----$ Ietrechloroethene & 10,14 \\
\hline $79-34-5 \cdots-\cdots-\cdots-1,2,2-$ Ietrachlereethane & 10.14 \\
\hline $108-88-3-\cdots-\cdots-10$ luene & 10,14 \\
\hline $108-90-7-\cdots-----$ Chlorobenzene & 10,11 \\
\hline $100-41-4-\cdots----$ Ethy lbenzene & 10,14 \\
\hline $109-42-5----\cdots--5$ syrene & 10,14 \\
\hline$\left|330-39-7-\cdots---x_{y}\right|$ ene (totei) & 10.14 \\
\hline
\end{tabular}


$\cdot$

IA

VOLATILE ORGANICS ANALYSIS DATA SHEET

EPA SAMPLE NO.

Lab NameiWestern Research Institut Contract:68-02-0017

Lab Codo:WRI Case No.:10201 SAS No.: 5 SDG No.:RM-1

Matrix: (soil/water) WATER

Lab Sample ID:

Sample wt/vol: $5.00(\mathrm{~g} / \mathrm{ml}) \mathrm{ML}$

Lab F1le ID: RMIOS

Level: (low/med) LOW

Date Recelved:12/10/92

X Molsture: not dec.

Date Analyzed:12/14/92

6C Column:DB-624

I0: $0.53(\mathrm{~mm})$

Dilution Factor: 1.0

Sold Extract Volume:0 (uL)

Sold Aldquot Volume:0

(uL)

CAS NO.

COMPOUND

CONCENTRATION UNITS:

(UQ/L or UQ/KO) UG/L $O$

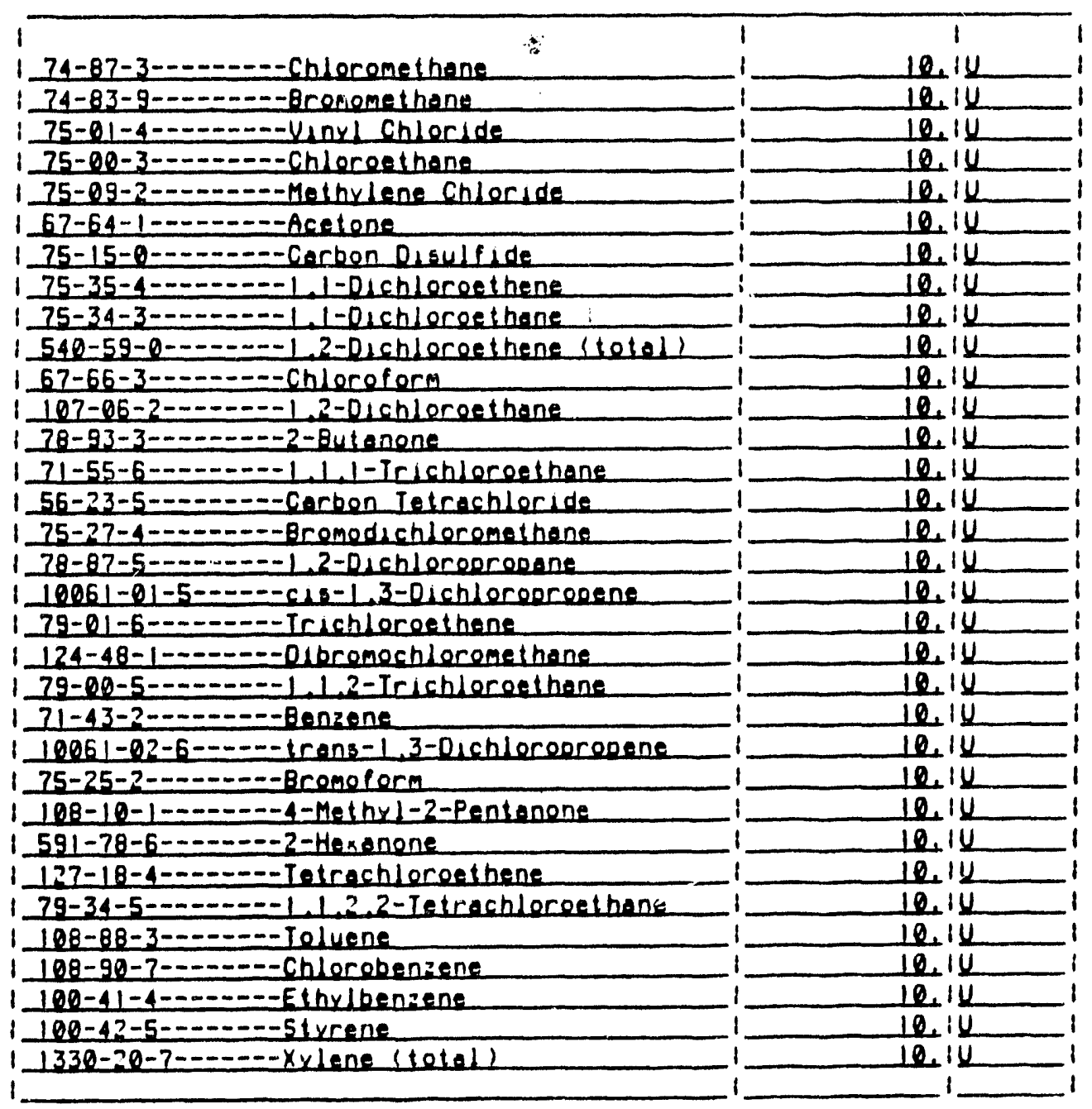


IA

VOLATILE ORGANICS ANALYSIS QATA SHEET

Lab Name:Western Research Institut

Contract168-02-0017

EPA SAMPLE No.

Lab Code:WRI Case No.110201 SAS No.: SOG No.:RM-1

Matrix: (soil/watar) WATER Lab Sample ID:

Sample wt/vol: $5.00(\mathrm{~g} / \mathrm{ml}) \mathrm{ML} \quad$ Lab File ID: RM108

Level: (low/med) Low

Date Received: $12 / 10 / 92$

X Moisture: not dec.

Date Analyzed: $12 / 14 / 92$

6C Column:DB-624 ID: $0.53(\mathrm{~mm})$

Difution Factor: $\quad 1.0$

Soil Extract Volume:0

(uL)

Sojl Aliquot Volume:0

(uL)

CAS NO.

COMPOUND

CONCENTRATION UNITS:

(UO/L or ug/Kg) UG/L Q

\begin{tabular}{|c|c|}
\hline & 1 \\
\hline $74-87-3-\cdots-\cdots-$ Chlor omethene & 10,14 \\
\hline $74-83-9-\cdots-\cdots--$ - Bromomethane & 10,14 \\
\hline $75-01-4-\cdots-----v_{i n y l}$ chloride & 10,11 \\
\hline $75-20-3-\cdots-\cdots-$ Chlereethene & 10,14 \\
\hline 75-99-2 - - - - - - Methylene Chloride & 10.14 \\
\hline $67-64-1 \cdots \cdots-\cdots-A c e t$ one & 10,14 \\
\hline $75-15-0-\cdots-\cdots-$ Cerbon DLsulfide & 10,14 \\
\hline $75-35-4 \cdots-\cdots---1,1-0$ ichloroethene & 10,11 \\
\hline $75-34-3-\cdots-----1,1-0$, chloroethane & 19,14 \\
\hline $540-59-\theta-\cdots-\cdots-1,2-0$ ichloroethene (totel) & 10,12 \\
\hline $67-66-3-\cdots-\cdots-$ chloroform & 10,10 \\
\hline 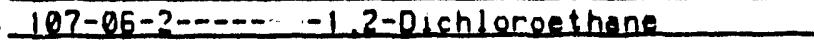 & 10,11 \\
\hline $78-93-3-\cdots \cdots---2-$ Butenone & 10,14 \\
\hline $71-55-6 \cdots \cdots-\cdots-1,1,1-$ Trichloreethene & 10,12 \\
\hline $56-23-5-\cdots-----$ Carbon Ieirachleride & 10,11 \\
\hline $75-27-4 \cdots \cdots-\cdots-$ - & 10,12 \\
\hline $78-87-5-\cdots-\cdots-1,2-0$ ichleropropene & 10,14 \\
\hline $10061-01-5-\cdots--c_{1}-1,3-0, a h l o r s$ & 10,14 \\
\hline 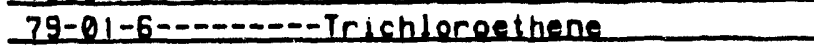 & $10+14$ \\
\hline $124-48-1-\cdots-\cdots-0$ ibromechloremethene & 10.14 \\
\hline $79-99-5---\cdots---1,1,2-$ Ir ichloresthene & 10,12 \\
\hline $71-43-2-\cdots-\cdots--1$ - Benzene & 10,12 \\
\hline $10061-02-6-\cdots-\cdots-1$ rans $-1,3-0$ ichlerooroos & 10,12 \\
\hline $75-25-2---\cdots---8$ comoform & 10,10 \\
\hline $108-10-1-\cdots \cdots-1-$ Methy 1-2-Pentenene & 10,14 \\
\hline $591-78-6-\cdots-\cdots--2-$ Hexinone & 10,14 \\
\hline $127-18-4-\cdots----$ Tetrechloroethene & 10.14 \\
\hline $79-34-5-\cdots-\cdots--1,1,2,2$-Ietrechlereethene & $10_{2}: 4$ \\
\hline $108-88-3-\cdots-----$ Toluene & 10.14 \\
\hline $108-90-7-------$ Chlorobenzente & 10,10 \\
\hline $100-41-4-\cdots-\cdots-$ Ethy 16 enrene & $1 \theta_{2} i x$ \\
\hline $100-42-5 \cdots-\cdots--5$ tyrene & $10, i 2$ \\
\hline $1330-20-7-\cdots--x_{y}$ lene (totol) & 10.14 \\
\hline
\end{tabular}


IA

VOLATILE ORGANICS ANALYSIS DATA SHEET

EPA SAMPLE NO.

Lab NameiWestern Research Institut Contract:68-02-0017 I EMWIIA

Lab Code:WRI Case No.:10201 SAS No.:

Matrix: (soll/water) WATER Lab Sample I0:

Sample wt/rol: $5.00(\mathrm{~g} / \mathrm{ml}) \mathrm{ML} \quad$ Lab Fale ID: RMIIG

Level: (low/med) LOW Date Received:12/10/92

X Moisture: not dec.

Date Analyzed:12/15/92

GC Column:DB-624 10: $0.53(\mathrm{~mm})$

Dilution Factor: $\quad 1.0$

Sold Extract Volume:o

(uL)

Soll Aliquot Volume:o

(uL)

CAS NO.

COMPOUND

CONCENTRATION UNITS:

(UO/L or Ug/Kg) UG/L Q

\begin{tabular}{|c|c|}
\hline $174-87-3-\cdots-\cdots--$ Chleromethane & 10.11 \\
\hline $74-83-9-\cdots-\cdots--8$ romome thane & 10,14 \\
\hline $75-01-4-\cdots-----v_{\text {inyl }}$ Chloride & 10,14 \\
\hline $75-90-3 \cdots--\cdots--$ Chloreethane & 10.iL \\
\hline 75-09-2---- - - - Methylene Chloride & 10.iv \\
\hline $67-64-1-\cdots-\cdots---$ Acetone & 10.14 \\
\hline $75-15-0-\cdots-----$ Carbon Disulfide & 10.14 \\
\hline $75-35-4 \cdots-\cdots---1,1-0, c h$ loreethene & 10.14 \\
\hline $75-34-3-\cdots-\cdots--1,1-0$, chlores thene & 10,14 \\
\hline $540-59-9-\cdots-\cdots-1,2-01 c h$ loroethene (tola) & 10.14 \\
\hline $67-66-3-\cdots-\cdots--$ Chloroform & 10.14 \\
\hline $107-06-3-\cdots----1,2-01$ chloresthone & 10,14 \\
\hline $18-93-3-\cdots-\cdots--2-8$ - 1 tenene & 10.11 \\
\hline $171-55-6-\cdots-\cdots-1,1,1-$ Irichloroethone & 10. 14 \\
\hline 56-23-5--------Carben. Ietrachloride & 10.14 \\
\hline $75-27-4-\cdots-\cdots--$ Bromodichloromethane & 10,14 \\
\hline $78-87-5-\cdots-\cdots-1,2-0$ ichloceproosene & 10.14 \\
\hline $10061-01-5-\cdots--c 15-1,3-016 h l o r o p r o p e n e$ & 10.11 \\
\hline $79-01-6-\cdots-----$ Ir lachloresthene & 10,14 \\
\hline $124-48-1-\cdots-\cdots-0$ ibromoch loromethane & 10,11 \\
\hline $179-00-5-\cdots-\cdots--1,1,2-$ Ir ichloresethane & 10.12 \\
\hline $71-43-2-\cdots-\cdots-$ - Benzene & 21 \\
\hline $10051-02-6-\cdots--1$ rans-1 3-Dichlecooroosene & 10,14 \\
\hline $75-25-2-\cdots-\cdots--$ Bromoform & 10,14 \\
\hline $108-10-1-\cdots \cdots--$ - Methy) - 2-Pentanone & 10.11 \\
\hline $591-78-6-\cdots-----2-$ Hexenene & 10,11 \\
\hline $127-18-4--\cdots---$ Tetrechloroethene & 10,14 \\
\hline $79-34-5 \cdots \cdots-\cdots-1,2,2$-Ietrachloroethene & 10,14 \\
\hline 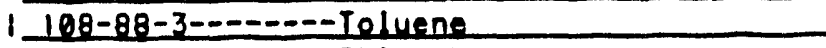 & 10.14 \\
\hline 108-90-7-------Chlorobenzene & 10.14 \\
\hline $109-41-4-\cdots----5$ - helbenzens & 10.14 \\
\hline $100-42-5-\cdots----5$ tyrene & 10,15 \\
\hline $1330-20-7-\cdots--x_{x}$ lene (totol) & 10.14 \\
\hline
\end{tabular}


IA

VOLATILE ORGANICS ANALYSIS DATA SHEET

EPA SAMPLE NO.

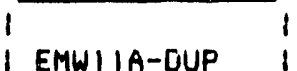

Lab Name: Western Research Institut Contract:68-02-0017!

Lab Code:WRI Case No.:10201 SAS No.:

Matrix: (soll/water) WATER

Lab Sample ID:

Sample wt/rol: $\quad 5.00(\mathrm{~g} / \mathrm{ml}) \mathrm{ML}$

Lab File ID: RMI25

Level: (low/med) LOW

Date Received: $12 / 10 / 92$

$x$ Mossture: not dec.

Date Analyzed:12/17/92

GC Column:DB-624

ID: $0.53(\mathrm{~mm})$

Dilution Factor: 1.0

Sold Extract Volume:0

(uL)

Soll Al lquot Volume:0

(uL)

CAS NO.

COMPOUND

CONCENTRATION UNITS:

(UO/L or UQ/Kg) UG/L $Q$

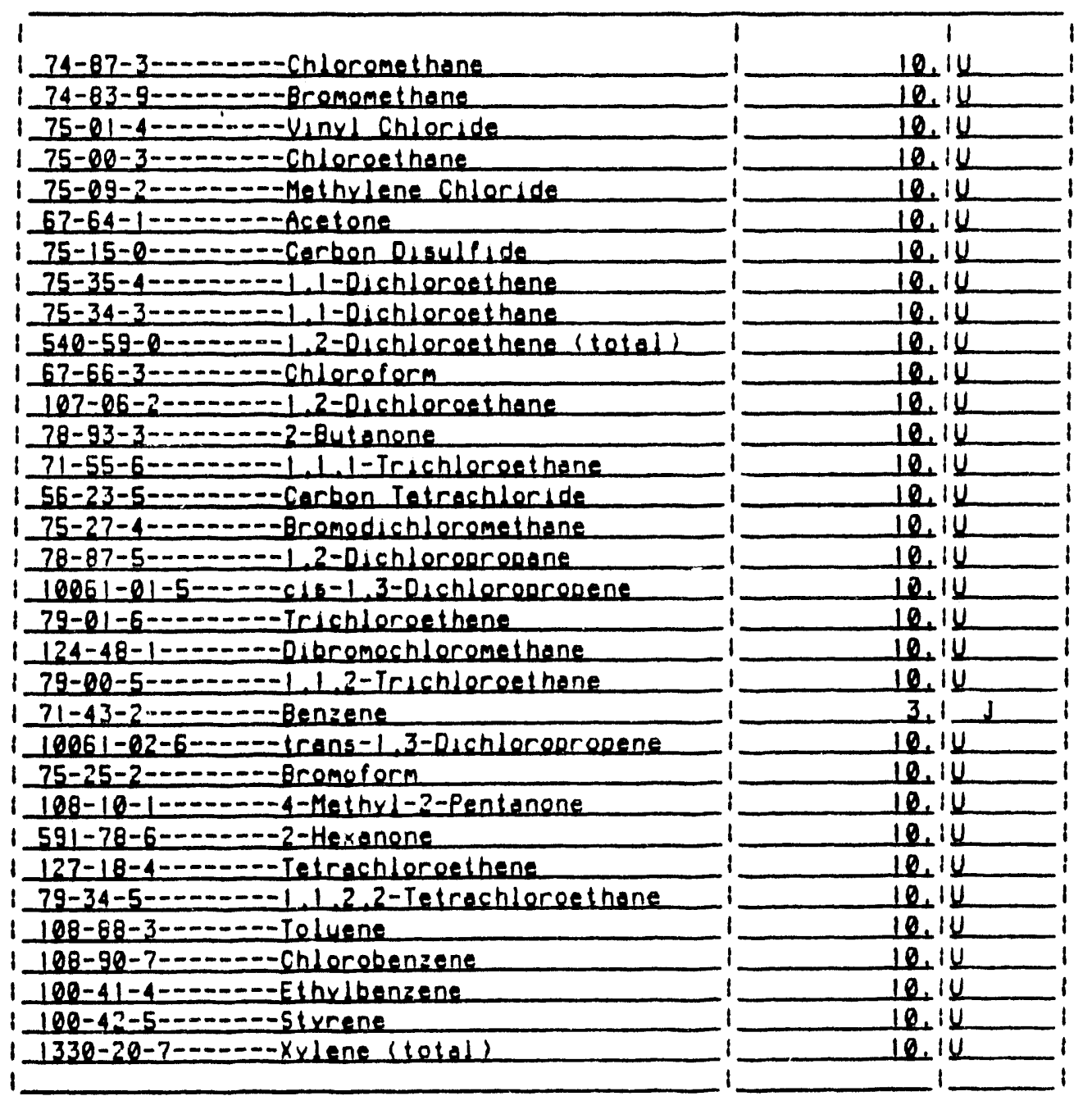


IA

VOLATILE ORGANICS ANALYSIS DATA SHEET

EPA SAMPLE NO.

$\begin{array}{ll}1 \text { TW2 } & 1 \\ 1\end{array}$

Lab Nameiwestern Research Instatut Contract:68-02-0017 1_

Lab CodeiwRI Case No.110201 SAS No.: SDG No. IRM-I

Matrix: (sosl/water) WATER Lab Sample ID:

Sample wt/rols $5.00(0 / m)$ ML Lab File ID: RMII5

Level: (low/med) Low Date Recelved:12/10/92

X Moisturar not dec. Date Analyzedi/2/15/92

6C Column:DB-624 ID: $0.53(\mathrm{~mm})$ Dilution Factor: 1.0

Soll Extract Volumeio (UL) Soil Aliquot Vulume:0 (UL)

CAS NO. COMPOUND $\left(\mathrm{ug}^{\mathrm{L}} \mathrm{L}\right.$ or $\left.\mathrm{ug} / \mathrm{Kg}\right) \mathrm{UG} / \mathrm{L} \quad \mathrm{Q}$

\begin{tabular}{|c|c|}
\hline i $24-87-3-\cdots-----$ Chloromethone & 10.11 \\
\hline 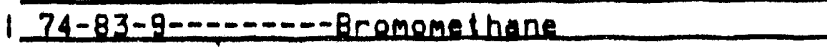 & 10,14 \\
\hline $75-01-4-\cdots----v_{\text {inyl chloride }}$ & 10,12 \\
\hline $75-00-3-\cdots-\cdots-$ Chloroethene & 10,14 \\
\hline 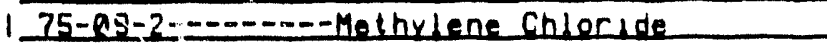 & 10,12 \\
\hline $67-64-1=\cdots-\cdots-\cdots$-Acetione & 10,14 \\
\hline $75-15-8 \cdots-\cdots--$ - cerbon Disulfide & 10.12 \\
\hline $25-35-4 \cdots \cdots-\cdots-1,1-0$, chloreethene & 10,12 \\
\hline 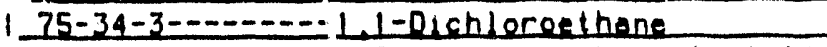 & 10,14 \\
\hline $540-59-0 \cdots \cdots-\cdots, 2-0$ ichloreethene (10tol) & 10,12 \\
\hline $67-66-3-\cdots \cdots$ bloreform & 10,10 \\
\hline $107-06-2-\cdots-\cdots-1$ 2-0ichloresthone & 10.11 \\
\hline $78-93-3-\cdots-\cdots--2-8 u t e n o n$ & 10.12 \\
\hline 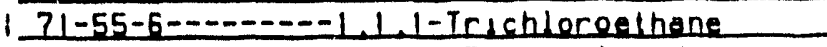 & 10.14 \\
\hline $56-23-5-\cdots-\cdots--c$ cerben Ietrachloride & 10,12 \\
\hline $75-27-4-\cdots-\cdots--B r e m e d i c h$ loromethone & 10.14 \\
\hline $78-87-5-\cdots-\cdots-1,2-0$ lchloropronene & 10,14 \\
\hline $10061-01-5-\cdots--c i s-1$ 3-Dichloreoreosene & 10.14 \\
\hline 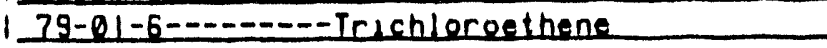 & 10.14 \\
\hline $124-48-1-\cdots-\cdots-0$ bromochloromethane & 10.112 \\
\hline 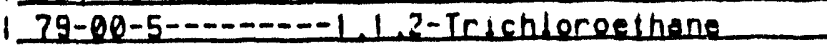 & 10.14 \\
\hline $71-43-2-\cdots \cdots-\cdots-$ Benzene & 10.11 \\
\hline $10061-02-6-\cdots--1$ cans -1 3-01chloropreoene & 10.11 \\
\hline $75-35-2-\cdots-\cdots-$ Bremoform & 10.14 \\
\hline $108-10-1-\cdots-\cdots-4-$ Methyl-2-Pentenone & 10.10 \\
\hline $591-78-6-\cdots-\cdots--2-$ Hexanone & 10,12 \\
\hline $127-18-4-\cdots---$-Ietrechleroethene & 10.11 \\
\hline $79-34-5 \cdots \cdots-\cdots-1,1,2-$ Tetrachleresthane & 10,11 \\
\hline $108-88-3-\cdots-\cdots-$ Ioluene & 10.14 \\
\hline $108-90-7-\cdots-\cdots-c h$ corobenzene & 10,12 \\
\hline $100-41-4-\cdots-\cdots-$ Ethy benzene & 10,14 \\
\hline $100-42-5-------5$ tyrene & 10,14 \\
\hline $1330-30-7------x_{x}$ lene (totel) & $10+11$ \\
\hline
\end{tabular}


IA

VOLATILE ORGANICS ANALYSIS DATA SHEET

EPA SAMPLE NO.

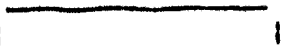

1 TW3

Lab NameiWestern Revearch Inatifut Contracti68-02-0017

Lab CodeIWRI

Case No.:10201

SAS No.:

SDG NO. IRM-1

Matrix: (soll/water) WATER

Lab Sample 10:

Sample wt / vol:

$5.00(\mathrm{~g} / \mathrm{ml}) \mathrm{ML}$

Lab File 10: RMII3

Level: (low/med) LOW

Date Recelved:12/10/92

* Molsture: not dec.

Date Analyzed:12/14/92

6C Column:08-624 I0:0.53(m)

Dilution Factor: $\quad 1.0$

Soll Extract Volume:0

(uL)

Sosl Alsquot Volume:O

(uL )

CAS NO.

COMPOUND

CONCENTRATION UNITS:

(UQ/L or UG/KO) UG/L Q

\begin{tabular}{|c|c|}
\hline $24-87-3-\cdots-\cdots-$ - Chloremethane & $\begin{array}{c}1 \\
10.14\end{array}$ \\
\hline $74-83-9-\cdots--\cdots-$ - Aromome thane & 10,10 \\
\hline 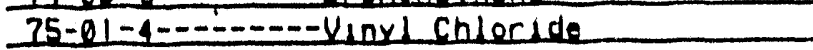 & 10.11 \\
\hline $75-09-3 \cdots \cdots-\cdots-c h l o r e=t h a n e$ & 10.1L \\
\hline 75-09-2-...- - Methylene chloride & 10,14 \\
\hline $67-64-1 \cdots-\cdots--$ Acetene & 10.14 \\
\hline $75-15-9 \cdots \cdots-\cdots-$ Corben Disulfide & 10,12 \\
\hline $25-35-4 \cdots \cdots--1,1-$ Dechlereethene & 10.12 \\
\hline $75-34-3-\cdots \cdots-1,1-0$ iahloreethene & 10,12 \\
\hline $540-59-\theta-\cdots-\cdots-1,2-0$ ichloreethene (toted) & 10.12 \\
\hline $67-66-3-\cdots-\cdots-$ Chloreform & 10.12 \\
\hline $107-06-2-\cdots-\cdots-1,2-01$ ch blereethene & 10,14 \\
\hline $78-93-3-\cdots \cdots-\cdots-2-8$ utenone & 10.11 \\
\hline $71-55-6 \cdots-\cdots-\cdots-1,1$, Ir icblereethent & 19.11 \\
\hline $56-23-5 \cdots \cdots-\cdots-$ Cerbon Tetrechlecide & 10.112 \\
\hline 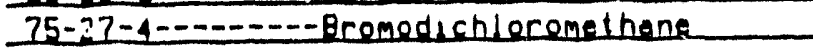 & 10.14 \\
\hline $78-87-5-\cdots-\cdots-1,2-0$, chloreoropene & 10.11 \\
\hline $10061-01-5-\cdots-c 10-1,3-01$ chloreorooene & 10.14 \\
\hline 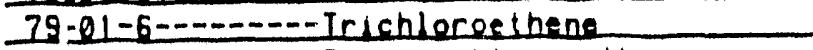 & 10.12 \\
\hline 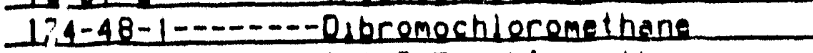 & 10,112 \\
\hline 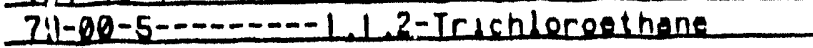 & 10.11 \\
\hline $71-43-2 \cdots \cdots-\cdots-$ Benzene & 21 \\
\hline $10061-02-6-\cdots-\cdots$ - rens -1 3-0 Lahberooreoene & 10.14 \\
\hline $75-25-2-\cdots-\cdots---8$ romoform & 10,14 \\
\hline $108-10-1-\cdots-\cdots-1-$ Methyl-2-Pentenone & 10,14 \\
\hline $591-78-6-\cdots \cdots--2-4$ exinene & 10,11 \\
\hline $127-18-1-\cdots-\cdots-$ Ietrechloresthene & 10,11 \\
\hline $29-34-5 \cdots \cdots-\cdots-1,2,2-I$ etrechlor & 10.11 \\
\hline $108-88-3-\cdots-\cdots-$ Ioluene & 10.11 \\
\hline $198-99-7-\cdots-\cdots-C h l e r o b e n z e n e$ & 10 iL \\
\hline $100-41-4-\cdots-\cdots-$ Elhylbenzene & 10,14 \\
\hline $109-42-5-\cdots-\cdots-5$ Syrene & 10,14 \\
\hline $1330-20-7-\cdots-x_{y}$ lene (totel) & $10+14$ \\
\hline
\end{tabular}


IA

VOLATILE ORGANICS ANALYSIS DATA SHEET

Lab Namei Western Research Institut Contract:68-02-0017

EPA SAMPLE NO.

Lab Code:WRI

Case No. 110201

SAS No. 1

Lab Sample ID:

Matrix: (soll/water) WATER

Sample wt/voll $5.00(\mathrm{~g} / \mathrm{ml}) \mathrm{ML}$

Level: (low/med) LOW

* Mossturei not dec.

6C Column:D8-624

ID: $0.53(\mathrm{~mm})$

Sold Extract Volume:0

(uL)

Lab File 10: RMII2

Dute Recelved:12/10/92

Date Analyzed/12/14/92

Didution Factori $\quad 1.0$

Soll Aliquot Volumeio

(UL)

CAS NO.

COMPOUND

CONCENTRATION UNITS:

(Ug/L or Ug/KO) UG/L Q

\begin{tabular}{|c|c|}
\hline $74-87-3-\cdots \cdots-c h$ cheromethene & 10.14 \\
\hline $74-83-9-\cdots-\cdots--$ Bromomethane & 10,11 \\
\hline $75-01-4 \cdots-\cdots--v_{10} \times 1$ chleride & 10.12 \\
\hline $75-90-3-\cdots \cdots--$ Chloreethone & 10,14 \\
\hline 25-09-2-- - - - - Methylene Chloride & 10,12 \\
\hline $67-64-1-\cdots-\cdots-\cdots-$ Acetone & 10.12 \\
\hline $75-15-8-\cdots-\cdots--$ Cerbon D2sulfide & 10.12 \\
\hline $75-35-4-\cdots-\cdots-1,1-D i c h l e r e s t h e n e$ & 10,112 \\
\hline $25-34-3-\cdots-\cdots--1,1-0$ ichloree thane & 10,12 \\
\hline $540-59-\theta \cdots \cdots--1,2-0$ lahloresthene (total) & 10.112 \\
\hline $62-66-3-\cdots=-\cdots-c$ chloroform & 10.14 \\
\hline $207-06-2-=-\cdots-1,2-0$ ichlereethane & 10,12 \\
\hline $28-93-3-\cdots-\cdots--2-8$ utonone & 10.11 \\
\hline $71-55-6-\cdots-\cdots-1,1,1-$ Ir dechlereethone & 10.11 \\
\hline $56-23-5-\cdots-\cdots--$ - Cacbon Ietrechleride & 10.12 \\
\hline $75-27-4-\cdots-\cdots--$-8romodichloremethene & 10.14 \\
\hline $78-87-5-\cdots-\cdots--1,2-0,1 c h$ loroorosene & 10,14 \\
\hline 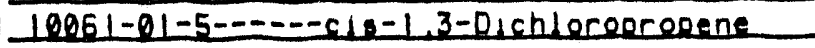 & $10+1 \bar{L}$ \\
\hline 79-91-6- - - - - - Irichloresthene & 10,11 \\
\hline $121-48-1-\cdots-\cdots-0$ - bromechloremethene & 10.14 \\
\hline 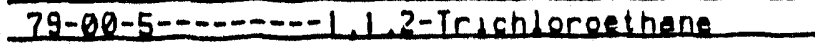 & $10+14$ \\
\hline $71-43-2-\cdots-\cdots-\cdots-8$ enzene & 19.14 \\
\hline $10961-02-6-\cdots-\cdots-1$ rens -1 3-01chlereoropene & 10,11 \\
\hline $25-25-2-\cdots-\cdots--8$ comoform & $10,1 \overline{1}$ \\
\hline $108-10-1-\cdots-\cdots-1-$ Methy L-2-Pentanone & $10, i \bar{L}$ \\
\hline $591-78-6-\cdots-\cdots-2-$ Hexpnone & 10,12 \\
\hline $127-18-4-\cdots-\cdots-$ Ietrechloroethene & $10,1 \overline{4}$ \\
\hline $79-34-5-\cdots-\cdots--1,1,2,2-$ Ietrechloreethene & $10+14$ \\
\hline $108-88-3 \cdots-\cdots--$ Ioluene & 10,14 \\
\hline $108-90-7-\cdots-\cdots-$ Chlorobenzene & $10+14$ \\
\hline |lag-41-4-------Ethylbenzens. & 10.14 \\
\hline $100-42-5-\cdots-\cdots-$ strene & 10,15 \\
\hline $1330-20-7-\cdots---x \times 1$ lene it & 10.12 \\
\hline
\end{tabular}


in

VOLATILE ORGANICS ANALYSIS DATA SHEET

Lab Nameitwastern Research Institut Contract:68-02-0017

Lab CodeIWRI

Case No.110201

SAS No.1

SDG No. IRM-1

Matrlxi (soll/water) WATER

Sample wt/vols $5.00 \mathrm{lo} / \mathrm{ml}) \mathrm{ML}$

Level: (low/med) LOW

X Molstures not dec.

6C ColumniD8-624

ID: $0.53(\mathrm{~mm})$

Soll Extract Volumeio

(uL)

COMPOUNO

CONCENTRATION UNITSI

(UO/L or UG/KO) UG/L $Q$

CAS NO.

Lab Sampla 10,

Lab File I01 RMIII

Date Receivedi12/10/92

Date Analyzedi/2/14/92

Didution Factor: $\quad 1.0$

Sold Aliquot Volumeio
EPA SAMPLE NO.

THS

(20)

romethane

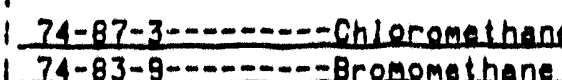

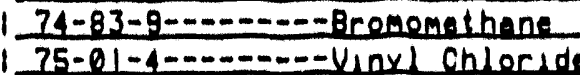

$75-00-3-\cdots-\cdots-c$ chlereethene

$175-09-2-\cdots-\cdots-\cdots$ Methrlene chloride

1 $67-64-1-\cdots \cdots-\cdots-$ Acetone

15-15-0--------Carbon Desulflde

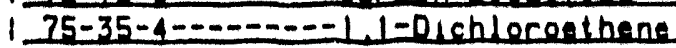

I $35-34-3 \cdots \cdots-\cdots-1,1-0$ ichlorenthene

$549-59-9 \cdots \cdots-1,2-0$ ichloresthene (totel

$67-66-3-\cdots-\cdots-c-c h l e r o f o r m$

$107-96-2 \cdots \cdots-1,2-01$ chlocosthene

$78-93-3-\cdots-\cdots--2-8$ utenone

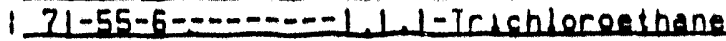

$156-23-5-\cdots-\cdots-C$ - Carbon Intrachloride

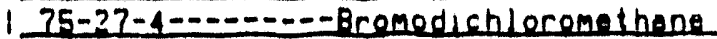

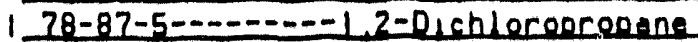

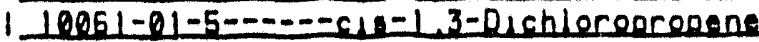

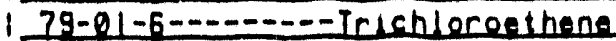

$124-48-1-\cdots-\cdots--D i b r e m e c h$ loromet hione

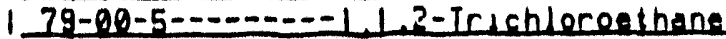

1. $11-43-2-\cdots-\cdots-\cdots-8$ enzene

$10961-02-6 \ldots-\cdots+$ trans $-1,3-0$ ichloroorooene

15-25-2-...-.--Bromoform

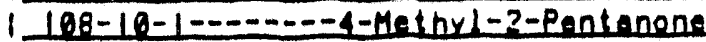

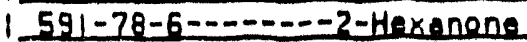

$127-18-4-\ldots----$ Ietrechloresthene

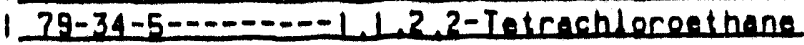

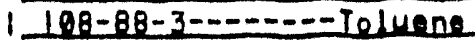

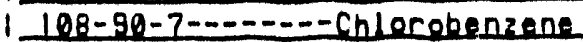

1 log-41-4------- Ethr|benzene

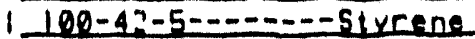

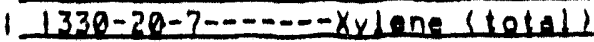

(uL)

\begin{tabular}{|c|c|}
\hline \multicolumn{2}{|c|}{10.14} \\
\hline$\frac{10}{10} 10$ & \\
\hline 10,14 & \\
\hline 10.14 & \\
\hline 10,14 & \\
\hline 10,14 & \\
\hline 10,14 & \\
\hline 10,14 & \\
\hline $10+11$ & \\
\hline 10,14 & \\
\hline 19.14 & \\
\hline 10.12 & \\
\hline 10.14 & \\
\hline 10.112 & \\
\hline 10.14 & \\
\hline 10.11 & \\
\hline 10.12 & \\
\hline 10.14 & \\
\hline 10.12 & \\
\hline 10.14 & \\
\hline 10,14 & \\
\hline 10.14 & \\
\hline 10.15 & \\
\hline 10.14 & \\
\hline 10.14 & \\
\hline 10.14 & \\
\hline 10.12 & \\
\hline 10.112 & \\
\hline 10.12 & \\
\hline 10.14 & \\
\hline 10.12 & \\
\hline 10,12 & \\
\hline 10.14 & \\
\hline
\end{tabular}


IA

VOLATILE ORGANICS ANALYSIS DATA SHEET

Lab NameiWestorn Rosearch Institut

Contract 168-02-0017

EPA SAMPLE NO.

Lab CodaIWRI

Cose No.110201

SAS No.I

SOG NO. IRM-1

Metrixi (100sl/water) WATER

Sample wt/voll $5.00(\mathrm{~g} / \mathrm{ml}) \mathrm{ML}$

Levels (low/mad) LOW

X Mosaturet not dec.

6C Columnio8-624

$1010.53(\mathrm{~mm})$

Sold Extract, Voluma 10

(uL)

CAS NO.

COMPOUND
Lab samplo ID:

Lab File IDI RMIOS

Date Recelvedi12/10/92

Date Analyzedi/2/14/92

Dsjution Factors $\quad 1.0$

Sosl Alsquot Volumero

(uL)

CONCENTRATION UNITSI

(UO/L or WO/KO) UG/L $Q$

\begin{tabular}{|c|c|}
\hline $74-87-3-\cdots-\cdots-\cdots-$ Chloromethane & $\begin{array}{c}1 \\
10.11\end{array}$ \\
\hline $74-83-9-\cdots-\cdots---8$ comemathane & 10,14 \\
\hline $25-01-1 \cdots \cdots-\cdots-v_{1 n} \times 1$ chloride & 10,11 \\
\hline $75-90-3-\cdots-\cdots-$ Chloreathene & 10.11 \\
\hline $75-99-2 \cdots \cdots-\cdots-$ Mathylene Chleride & 10.14 \\
\hline $62-64-1 \cdots \cdots \cdots-$ Acetone & 19.11 \\
\hline $75-15-9-\cdots \cdots-\cdots-$ Cacbon Disulfide & 10,11 \\
\hline $75-35-1-\cdots-\cdots--1,1-0$ iablereethene & 19.14 \\
\hline $75-34-3 \cdots \cdots-\cdots-1,1-0$, chloreathene & 19.12 \\
\hline $549-59-9-\cdots----1,2-0$ lachlores thene (lotal) & 10,11 \\
\hline $67-56-3-\cdots \cdots-c$ Chloreform & 10,11 \\
\hline $102-06-2 \cdots-\cdots-1,2-0$ candoconthane & 10,11 \\
\hline $78-93-3-\cdots \cdots-\cdots-2-8$ uteneng & 10,12 \\
\hline $71-55-6 \cdots-\cdots-\cdots-1,1,1-$ Ir lachleresthene & 10,14 \\
\hline $56-23-5-\cdots-\cdots--$ - Carbon Intrachlorde & 19.1U \\
\hline $75-27-4 \cdots-\cdots---8$ comodichloromethent & 10,12 \\
\hline 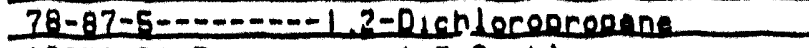 & 19.11 \\
\hline 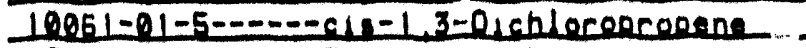 & 10.14 \\
\hline 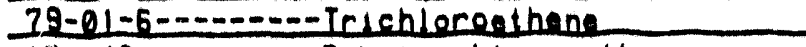 & 19.11 \\
\hline $124-48-1-\cdots-\cdots-0$ dbromochloromethane & 10,14 \\
\hline 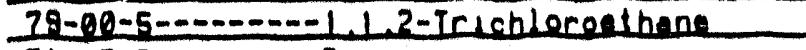 & 10.14 \\
\hline $21-43-2-\cdots \cdots-\cdots$ - Benzene & 19,11 \\
\hline $10061-02-6-\cdots---\operatorname{trans}-1$ 3-01ahlereoropene & 10,11 \\
\hline $75-25-2-\cdots \cdots-\cdots-$ - iromeform & 10,14 \\
\hline $108-10-1 \cdots \cdots-1-$ Mathry $1=2-$ Pen & 19.14 \\
\hline $581-78-5-\cdots-\cdots--2-$ Hexenene & 19,11 \\
\hline $127-18-1-\cdots-\cdots-$ Ietrachleresthene & 19,14 \\
\hline $29-34-5-\cdots-\cdots-1,{ }^{2}, 2-I$ - treahlorenthene & 19,14 \\
\hline $128-88-3 \cdots \cdots-\cdots-$ Ioluene & 10,112 \\
\hline $108-99-7-\cdots-\cdots-C h$ lorobenzene & 10.11 \\
\hline 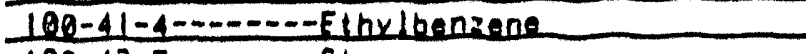 & 19.11 \\
\hline $199-42-5-\cdots----5$ Strene & 10.112 \\
\hline $1330-30-7 \cdots-\cdots-x \times 1$ ene 180 & \\
\hline
\end{tabular}


volatille organics analysis data sheEt

Lab Nameitwostorn Rosearch institut Contracti68-02-0017

EPA SAMPLE NO.

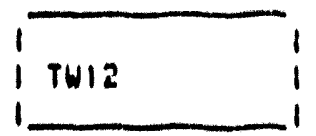

Lab COdeiWRI Case No.110201 SAS No.1

SDG No. IRM-1

Matrint (soll/wator) WATER

Sample wt/voll $5.00(\mathrm{~g} / \mathrm{ml}) \mathrm{ML}$

Levell (low/med) LOW

X Mossturas not dac.

GC ColumnioB-624

$1010.53(\mathrm{~mm})$

Soll Extract Volumeio

(ul)

COMPOUND

CAS NO.
Lab Sample 10:

Lab File IDI RMIOE

Dete Recesvedi12/10/92

Data Analyzedi/2/14/92

Dilution Factors $\quad 1.0$

Soll Aliquol Volumeio

(uL)

CONCENTRATION UNITSI

(UG/L or UO/KO) UG/L O

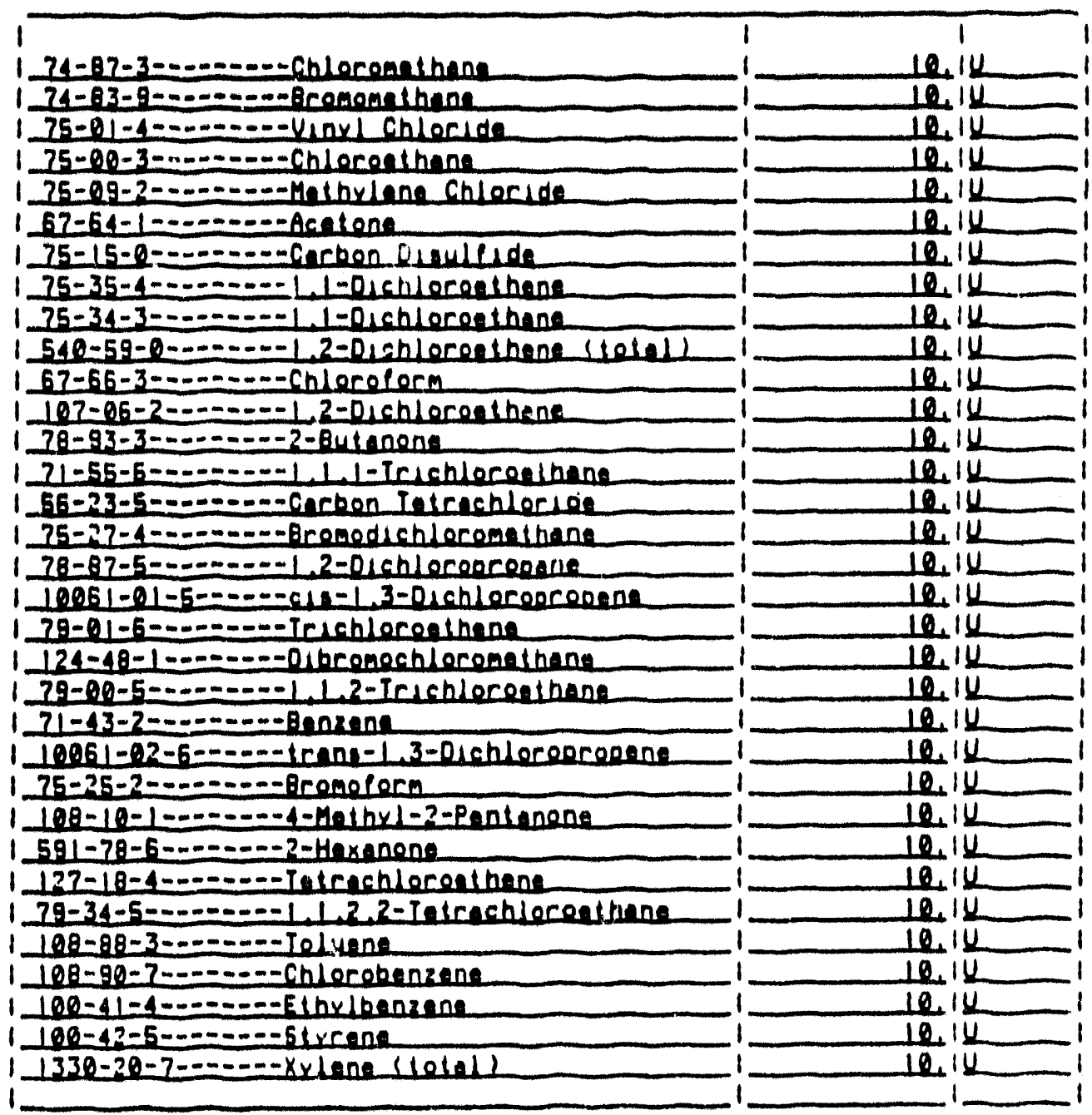


in

VOLATILE ORGANICS ANALYSIS DATA SHEET

Lab Namaiwastern Revearch Inatitut

Lab CodaIWR!

Case No.110201

Motrixl' (sold/wator) WATER

Semple wt/rolt

$5.00(0 / \mathrm{ml}) \mathrm{ML}$

Levald (low/mad) LoW

$x$ Moloturat not dac.

$6 C$ ColumniDA-624

$1010.53(\mathrm{~mm})$

Sodl Extract Volumeio

(uL)

SAS No.1

SDG NO. IRM-1

Lab Samplo I0:

Lab File IDI RMIOT

Dote Rocesvedi12/10/82

Data Anolyzedi12/14/92

Ollution Factors $\quad 1.0$

Sold Allquot Volumeio

(uL)

CAS NO.

COMPOUND

CONCENTRATION UNITSI

(UO/L or UQ/KO) UG/L O

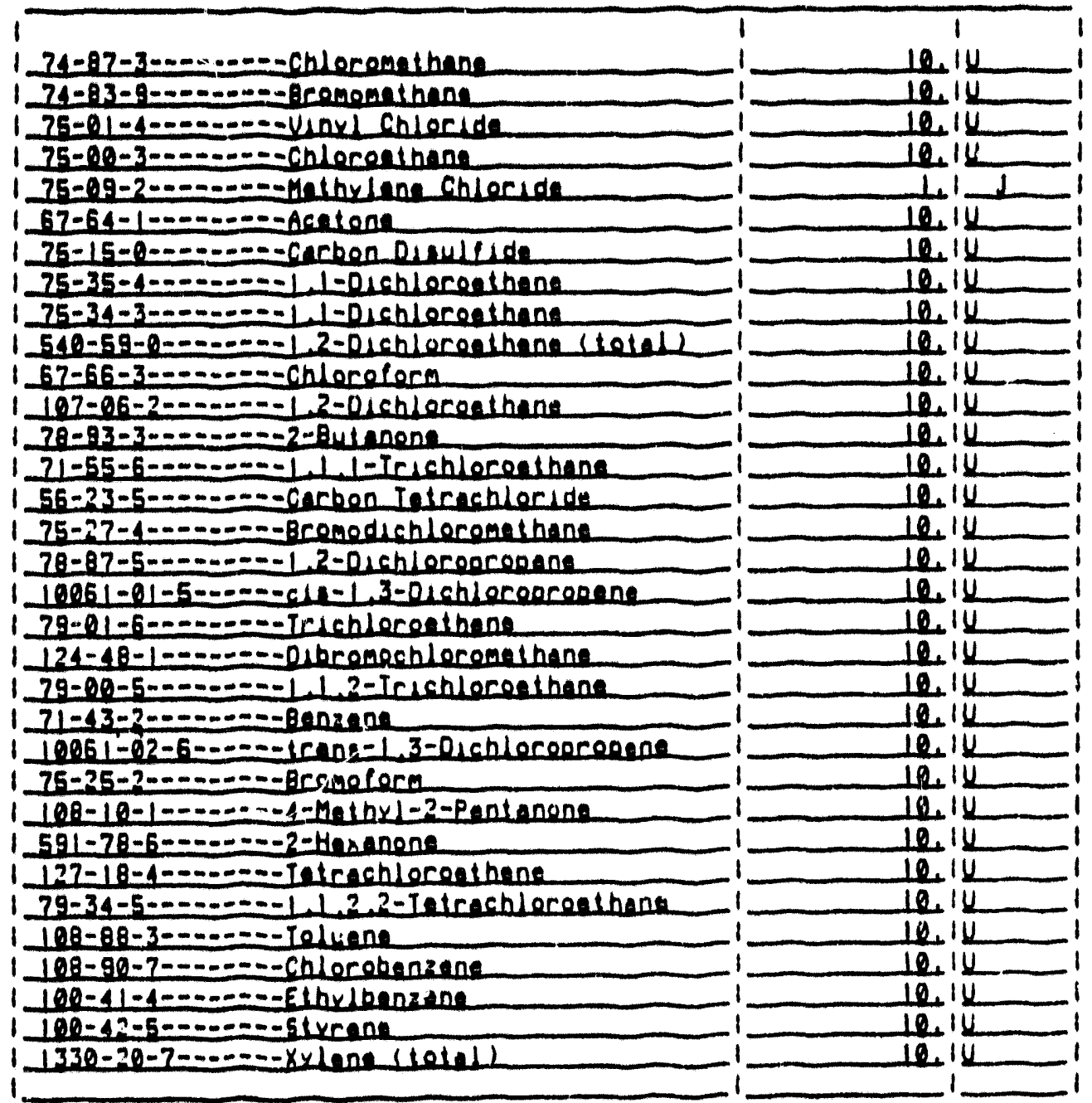


VOLATILE ORGANICS ANALYYSIS DATA SHEET

EPA SAMPLE NO.

1

1 TWIS

Lab Nameitwastorn Raseerch Inatstut Contracti68-02-0017

Lab CodalWRI Case No.110201 SAS No.1 SOG NO.IRM-1

Matrixt (sold/water) WATER Lab Sample IDi

Sample wt/vols 5.00 (o/ml) ML Lab File IOI RMIOI

Loveld (low/mod) LOW

Date Receivedi12/10/92

$x$ Modotures not dac.

Date Analyzedi12/14/92

6C Columnido-624

$10,0.53(\mathrm{~mm})$

Dilution Factors $\quad 1.0$

Sold Extract Volumeio (UL)

Soll Aliquot Volumeio

(uL)

CAS NO.

COMPOUNO

CONCENTRATION UNITS:

(Ug/L or UQ/KO) UG/L. 0

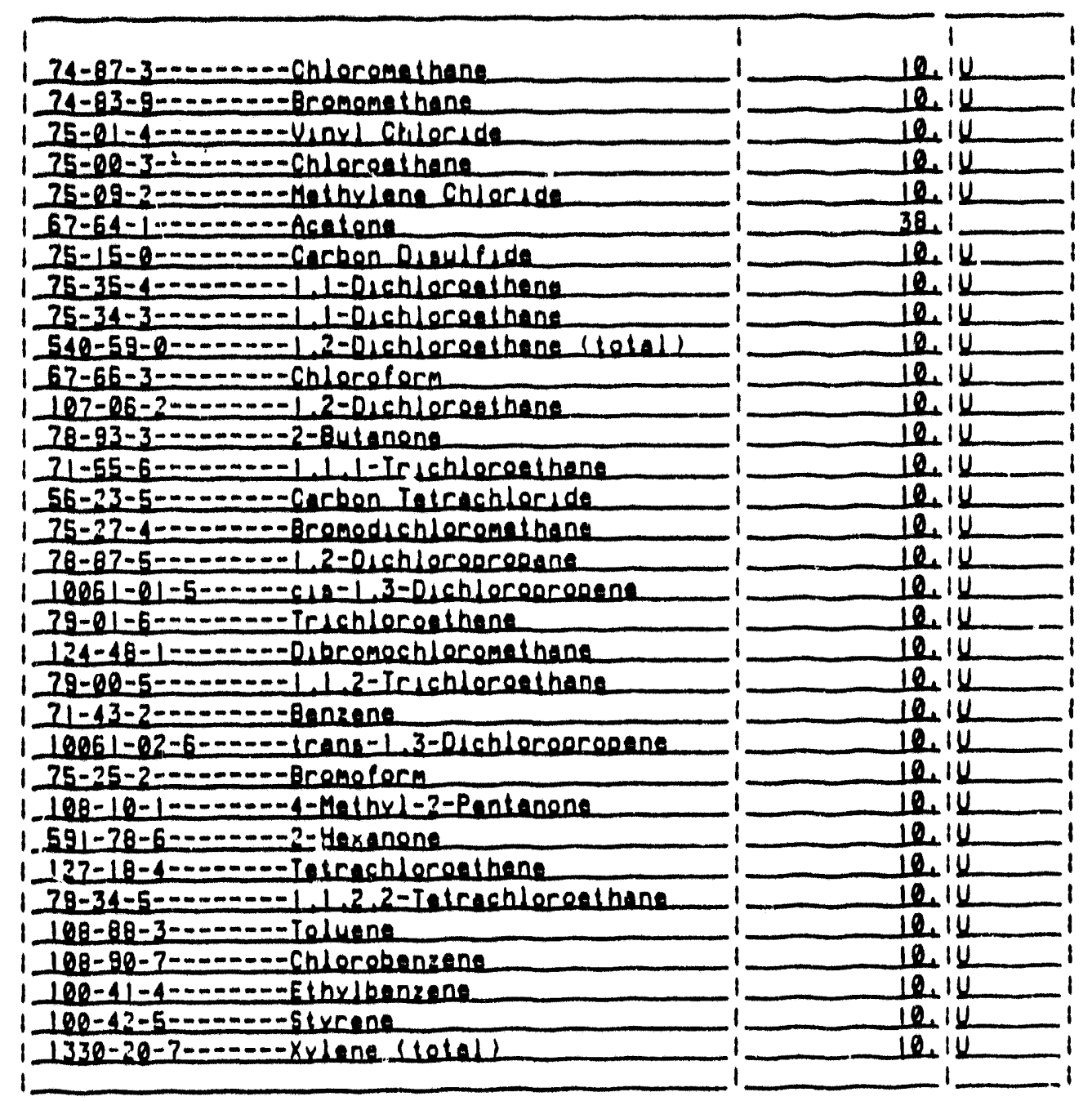


in

VOLATILE ORGANICS ANALYSIS DATA SHEET

EPA SAMPLE NO.

Lab Nameswastern Rasearch Institut Contracti68-02-0017

Lab CodaiWRI

Case No.110201

SAS No.1

SDG No. IRM-1

Matrixi (Boillwatar) WATER

Sample wt/val: $5.00(0 / \mathrm{ml}) \mathrm{ML}$

Level: (low/med) LOW

X Mosstures not dec.

GC ColumniD8-624

10. $0.53(\mathrm{~mm})$

(UL)

Sosd Entract Volumeio

Lab sample 10:

Lab File ID, RMIOZ

Dote Recelvads 12/10/92

Deto Analyzedi12/14/92

Didution Factor: $\quad 1.0$

Soll Allquot Volumeio

(uL)

CAS NO. COMPOUND (ug/L or ug/Kg) UG/L O

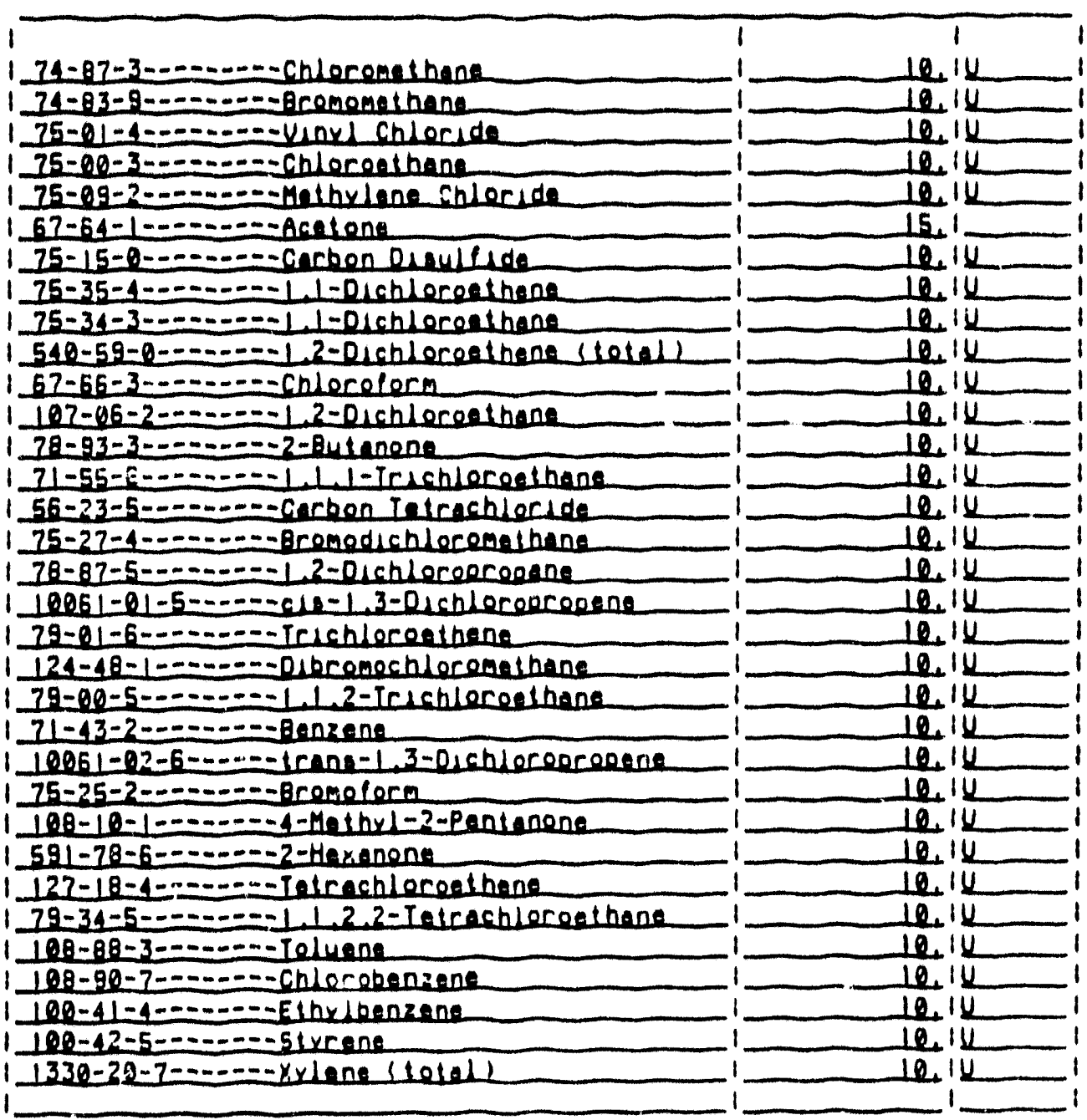


IA

VOLATILE ORGANICS ANALYSIS DATA SHEET

Lab Name: Western Research Institut Contract:68-02-0017

Lab Code:WRI Case No.:10201 SAS No.:

Matrixi (soil/water) WATER

Sample wt/rol:

$5.00(\mathrm{~g} / \mathrm{ml}) \mathrm{ML}$

Level: (low/med) LOW

X Moisture: not dec.

GC Column:DB-624

ID: $0.53(\mathrm{~mm})$

Soil Extract Volume:0

(UL)

COMPOUND

CAS NO.
Lab Sample ID:

Lab F1le ID: RM103

Date Recelvedi12/10/92

Date Analyzedi12/14/92

Dilution Factor: $\quad 1.0$

Soil Aliquot Volume:0

(uL) CONCENTRATION UNITS: (UO/L or UQ/Kg) UG/L

Q

\begin{tabular}{|c|c|}
\hline $74-87-3-\cdots---1-$ Chloromethene & $\begin{array}{r}1 \\
10.14\end{array}$ \\
\hline $74-83-9-\cdots-\cdots-$ Bromomethene & 10,10 \\
\hline $75-01-4-\cdots------$ Vinyl chloride & 10,14 \\
\hline $75-00-3-\cdots------$ chloroethane & 10.11 \\
\hline $75-09-2-\cdots---$ Methylene chleride & 10,14 \\
\hline $67-64-1-\cdots-\cdots-\cdots-$ Acetone & 10,14 \\
\hline $75-15-0-\cdots-----$ Cerbon oisulfide & 10,11 \\
\hline $75-35-4-\cdots-\cdots---1,1-0$ ichlereethene & $10+12$ \\
\hline $75-34-3-\cdots-----1,1-0$, chloresthane & 10.14 \\
\hline $54 \theta-59-\theta-\cdots----1,2-0$ ichlor oethene (totol) & 10,14 \\
\hline $67-66-3-\cdots-----$ chloroform & 10.14 \\
\hline $107-06-2-\cdots----12-0$ ichloreethane & 10,10 \\
\hline $78-93-3-\cdots----2-8$-8utenone & 10,14 \\
\hline $71-55-6-\cdots-\cdots--1,1,1-$ Irichloreethene & 10,14 \\
\hline i $56-23-5-\ldots-\cdots--$-Carbon Ietrachleride & 10.14 \\
\hline $75-27-4-\cdots-\cdots--1$ romodichloromethene & 10,14 \\
\hline $78-87-5-\cdots---1,2-0$ ichlereoropene & 10.14 \\
\hline $10061-01-5-\cdots---0 / 5-1,3-0$ ichlorooropene & 10,14 \\
\hline $79-01-6-\cdots--\cdots--$-Trichloroethene & 10,14 \\
\hline $124-48-1-\cdots-\cdots-n$ n bromechloromethene & 10,11 \\
\hline $79-9 \theta-5-\cdots \cdots$, , 2 -Irichloroethone & 10.14 \\
\hline $71-43-2-\cdots-----$ Benzene $_{1}$ & 10.14 \\
\hline $10061-02-6-\cdots--\operatorname{trans}-1$ 3-0,ichloreoropene & 10.14 \\
\hline $75-25-2-\cdots--1-$ Bromoform & 10,14 \\
\hline $108-10-1--------4-$ Methyl-2-Pentenone & 10,14 \\
\hline $591-78-6-\cdots----2-$ Hexenene & 10.14 \\
\hline $127-18-4-\ldots----$ Tetrechloreethene & 10.14 \\
\hline $79-34-5-\cdots-\cdots--1,2,2$-Tetrechloreethene & 10.14 \\
\hline $188-88-3-------$ Ioluene & 10,14 \\
\hline $108-90-7-\cdots-\cdots--C h l o r o b e n z e n e$ & $19+14$ \\
\hline $100-41-4-\cdots----$ Ethribenzene & $11 ?+14$ \\
\hline $100-42-5-\cdots-\cdots---5$ tyrene & -14 \\
\hline $1330-20-7-\cdots---x_{y}$ lene (totell) & $19+4$ \\
\hline
\end{tabular}


IA

VOLATILE ORGANICS ANALYSIS DATA SHEET

EPA SAMPLE NO.

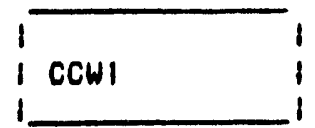

Lab Name: Western Research Institut

Contract: 68-02-0017

Lab Code:WRI Case No.:10201 SAS No.: SDG No.:RM-1

Matrix: (soll/water) WATER Lab Sample ID:

Sample wt/vol: $5.00(\mathrm{~g} / \mathrm{ml}) \mathrm{ML}$ Lab File ID: RMI2I

Level: (low/med) LoW

Date Recelved:12/10/92

X Moiature: not dec.

Date Analyzed:12/15/92

GC Column:DB-624

I0: $0.53(\mathrm{~mm})$

D: Sution Factor: 1.0

Soll Extract Volume:0

(uL)

Soll Aliquot Volume:0

(ul. )

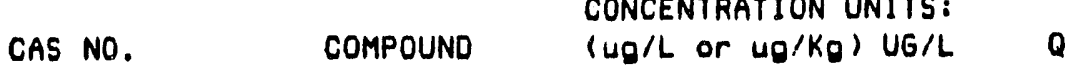

\begin{tabular}{|c|c|}
\hline | $74-87-3-\cdots----$-Chloromethane & 10.11 \\
\hline $74-83-9-\cdots-----8$ romomethane & 10,15 \\
\hline $75-01-4 \cdots-\cdots--v_{\text {inyl }}$ chloride & 10.11 \\
\hline $75-00-3-\cdots-\cdots-c$ chloroethane & 10.11 \\
\hline 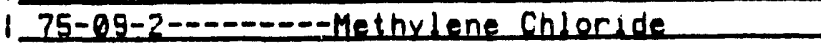 & 10,11 \\
\hline $67-64-1-\cdots-\cdots--$ Acetone & 19.iL \\
\hline $75-15-0-\cdots-----$ - erbon Disulfide & 10,112 \\
\hline $75-35-4-\cdots--\cdots-1,1-0$ ichloresthene & 10.14 \\
\hline $75-34-3-\cdots-\cdots--1,1-0$ ichloroethene & 10,12 \\
\hline $540-59-0-\cdots-\cdots-1,2-01$ chlorosthene (total) & 10,15 \\
\hline $67-66-3-\cdots-\cdots-c h l o r e$ form & 10,14 \\
\hline $107-05-2 \cdots \cdots-\cdots-1,2-0$ ichloroethane & 10,14 \\
\hline $78-93-3-\cdots-\cdots---2-8$ utanene & 10.11 \\
\hline $71-55-6 \cdots \cdots-\cdots-1,1-\operatorname{Tr}$ ichloroethane & 10.14 \\
\hline $56-23-5 \cdots-\cdots--$-cerbon Tetrachlor ide & 10.14 \\
\hline $75-27-4-\cdots-\cdots---$ Bromodichloromethone & 10.14 \\
\hline $78-87-5-\cdots \cdots--1,2-0$, chloroorooene & 10.14 \\
\hline $10061-01-5-\cdots--015-1,3-01$ chloreorooene & 10.14 \\
\hline 79-01-6-- - - - - Triahloreethene & 10.14 \\
\hline $124-48-1 \cdots \cdots-\cdots$ - ibromochloromethane & 10,14 \\
\hline $29-90-5-\cdots-\cdots--1,1,2-$ Trichloreethene & $10, i \bar{y}$ \\
\hline $71-43-2 \cdots-\cdots--$ - Benzene & $10 . i \overline{1}$ \\
\hline $10061-02-6-\cdots---$ trans -1 3-Dichloroorogene & 10.iL \\
\hline $75-25-2-\cdots-----$ Bromoform & $10,1 \overline{1}$ \\
\hline $108-10-1-\cdots-----4-$ Methx1-2-Pentenene & 10.11 \\
\hline $591-78-5-\cdots-\cdots--2-$ Hexanone & 10.11 \\
\hline $127-18-4-------$ Ietrachloroethene & 10,11 \\
\hline 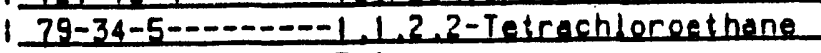 & 10.11 \\
\hline $108-88-3-\cdots----$ Ioluene & 10,15 \\
\hline $108-90-7-\cdots---c$ - chlorobenzene & 10.14 \\
\hline $100-41-4-\cdots----5$ Ethylhenzene & 10.12 \\
\hline $100-42-5-\cdots----5$ s 1 rene & 10,112 \\
\hline $1330-30-7-\cdots---x$ y lene (totol) & $10 . i 4$ \\
\hline
\end{tabular}


IA

VOLATILE ORGANICS ANALYSIS DATA SHEET

Lab Name:Western Research Institut

Lab CodeiWRI

Case No.110201

SAS NO.:

Contract: 68-02-0017

Matrix: (soll/water) WATER

Sample wt/vol: $5.00(\mathrm{~g} / \mathrm{ml}) \mathrm{ML}$

Level: (low/med) LOW

X Molsture: not dec.

GC Column:08-624 ID: $0.53(\mathrm{~mm})$

Soil Extract Volumeio

(uL)
EPA SAMPLE NO.

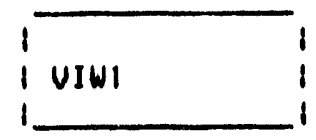

SDG No.:RM-1

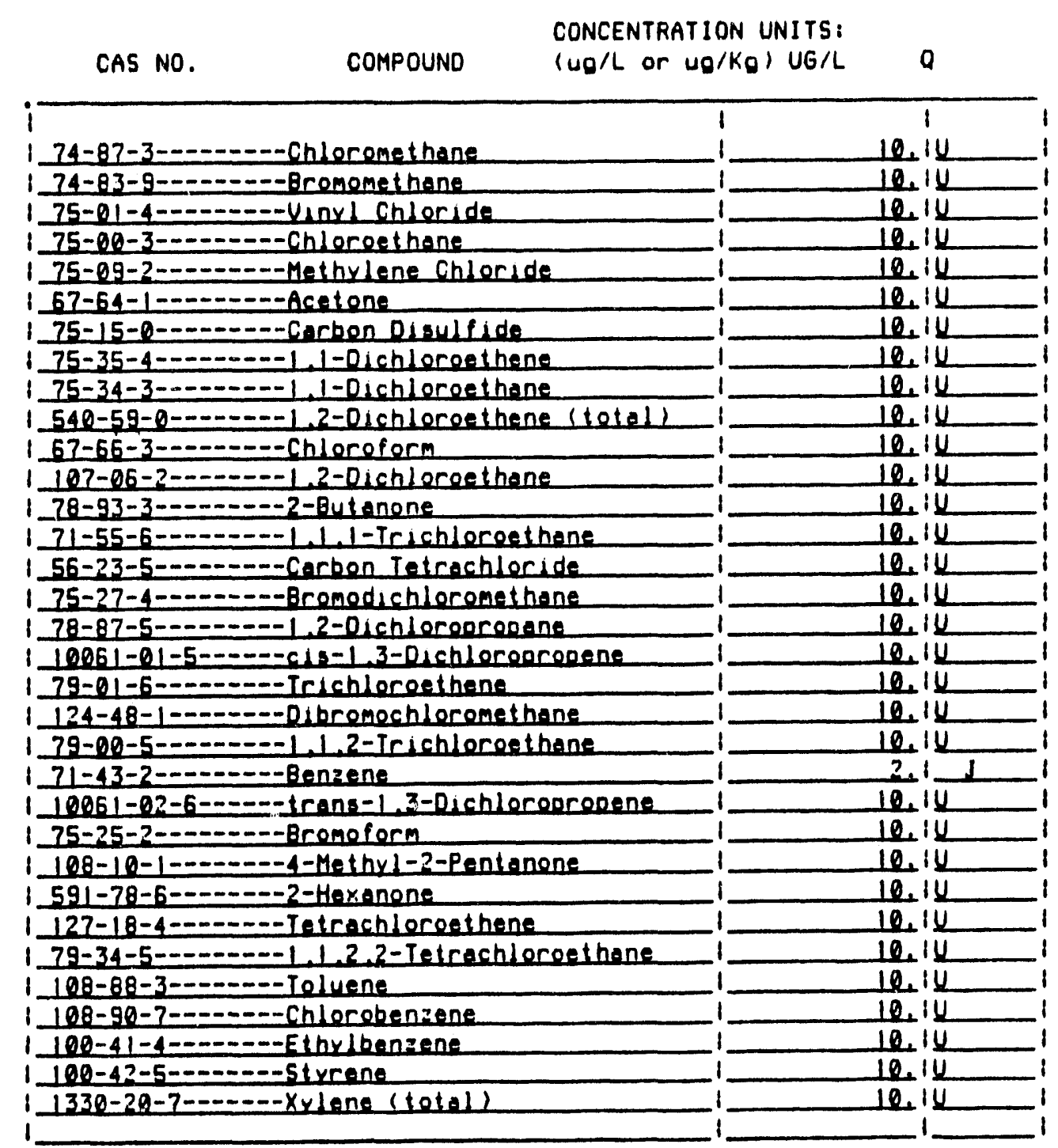


IA

VOLATILE ORGANICS ANALYSIS DATA SHEET

EPA SAMPLE NO.

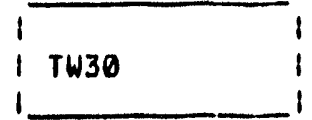

Lab Nameiwestern Research Institut Contracti68-02-0017 I

Lab CoderWRI

Case No.110201

SAS NO.,

SDG No. RRM-1

Matrin: (soll/water) WATER

Sample wt/vol: $5.00(\mathrm{~g} / \mathrm{ml}) \mathrm{ML}$

Level: (low/med) LOW

X Moistures not dec.

6C ColumnidB-624

ID: $0.53(\mathrm{~mm})$

Soll Extract Volume:0

(uL)

CONCENTRATION UNITS:
COMPOUND
(Ug/L or $u_{g} / \mathrm{Kg}$ ) UG/L $Q$
CAS NO.

Lab Sample ID:

Lab File ID: RM104

Date Received:12/10/92

Date Analyzed: 12/14/92

Dilution Factor: 1.0

Soll Allquot Volume:0 (UL)

Leromethone

I $74-87-3-\cdots \cdots--$ Chleromethene

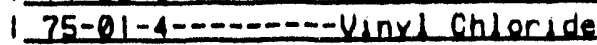

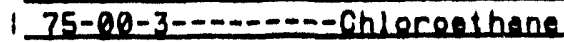

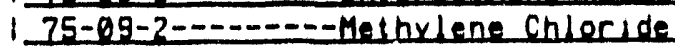

$67-64-1-\cdots-\cdots-$ Acetone

i $75-15-0-\cdots-\cdots--$ - Cerbon Disulfide

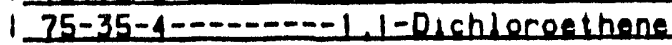

$175-34-3-\cdots \cdots--1,1-0$, chleree thene

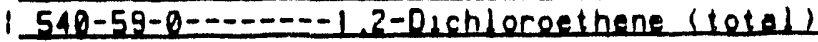

157-66-3-...----chloroform

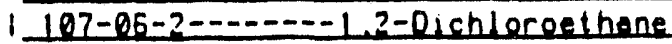

$178-93-3-\cdots-\cdots-\cdots-2-8$ utenone

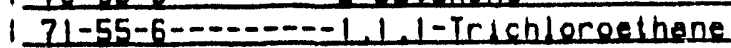

$156-23-5-\cdots-----$ - Carbon Ietrechloride

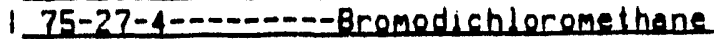

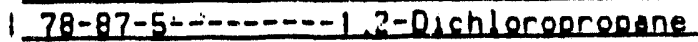

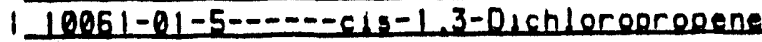

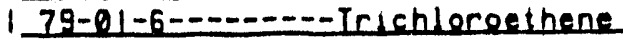

$124-48-1-\cdots---01$ - 1 romochloromethone

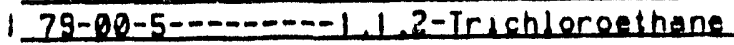

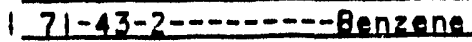

$10961-02-6-----$ trens $-1,3-0$ ich Lereorooene

75-25-2-- - - - - Bromoform

$108-10-1-\cdots-\cdots-4-$ Methyl-2-Pentenene

$1591-78-6-\cdots-\cdots--2-$ Hexenone

$127-18-4-\cdots----$ Ietrachleroe therie

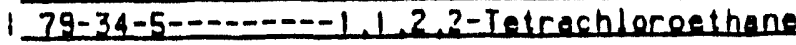

1 198-88-3------- I0 luene

1 108-90-7--------Chlor obenrene

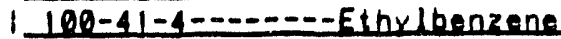

$109-42-5-\cdots-\cdots-51$ yrene

$1330-2 \theta-7 \cdots-\cdots-x y$ lene (total)

\begin{tabular}{|c|c|}
\hline & 1 \\
\hline$\frac{10.14}{10.10}$ & \\
\hline 10.14 & \\
\hline 10.14 & \\
\hline 10.14 & \\
\hline 10.12 & \\
\hline 10.14 & \\
\hline 10.14 & \\
\hline 10.14 & \\
\hline 10.14 & \\
\hline 10.14 & \\
\hline 10.14 & \\
\hline 10.12 & \\
\hline 10.14 & \\
\hline 10,14 & \\
\hline 10.12 & \\
\hline 10.14 & \\
\hline 10.12 & \\
\hline 10.14 & \\
\hline 10.14 & \\
\hline 10,14 & \\
\hline 120.1 & \\
\hline 10.14 & \\
\hline 10.14 & \\
\hline 10.14 & \\
\hline 10.14 & \\
\hline 10.14 & \\
\hline 10,14 & \\
\hline 10.14 & \\
\hline 10.14 & \\
\hline 10.14 & \\
\hline 10.14 & L \\
\hline 10.14 & $\underline{L}$ \\
\hline
\end{tabular}


IA

VOLATILE ORGANICS ANALYSIS DATA SHEET

EPA SAMPLE NO.

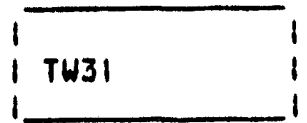

Lab NameiWestern Research Institut Contracti68-02-0017 I__

Lab Code:WRI Case No.:10201 SAS No.1 SOG No.:RM-1

Matrixi (soil/water) WATER

Sample wt/rol: $5.00(\mathrm{~g} / \mathrm{ml}) \mathrm{ML}$

Level: (low/med) LOW

X Moisture: not dec.

GC Column:08-624

ID: $0.53(\mathrm{~mm})$

Soll Extract Volume:0

(uL)

CAS NO. COMPOUND (Ug/L or UO/KQ) UG/L Q

Lab Sample ID:

Lab File ID: RMI20

Date Recelved:12/10/92

Date Analyzedi12/15/92

Dilution Factor: $\quad 1.0$

CONCENTRATION UNITS:

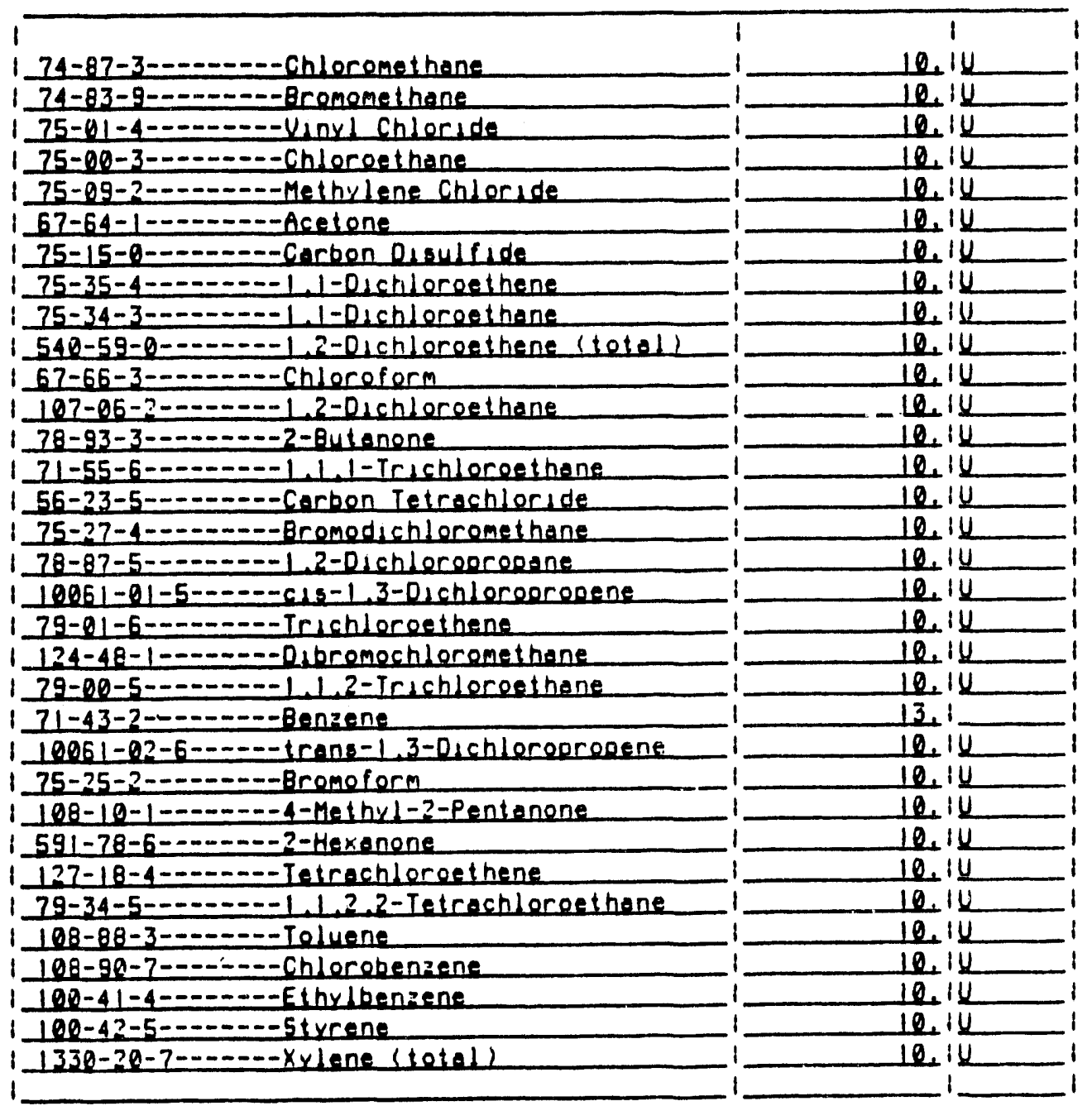




\section{Lab NameiWestern Research Institut \\ Lab CodeiWRI \\ Case No.:10201 \\ Matrix: (soll/water) WATER \\ Sample wt/roll $5.00(\mathrm{~g} / \mathrm{ml}) \mathrm{ML}$ \\ Level: (low/med) LOW}

X Moisturei not dec.

6C Column:DB-624

ID: $0.53(\mathrm{~mm})$

Sold Extract Volume:0

(uL)

Contract:68-02-0017

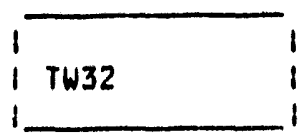

SAS NO.:

SDG No.:RM-1

Lab Sample ID:

Lab File 1D: RMI23

Date Recesved:12/10/92

Date Analyzedi12/15/92

Dilution Factor: $\quad 1.0$

Soll Al iquot Volumeio

(uL)

CONCENTRATION UNITS:

CAS NO. COMPOUND $(u g / L$ or $u g / K g) U G / L \quad Q$

\begin{tabular}{|c|c|}
\hline I $74-87-3-\cdots-\cdots-c h$ loromethane & 10.11 \\
\hline $74-83-9-\cdots-----8$ romomethene & 10.10 \\
\hline $75-\theta \mid-4-\cdots-\cdots--(1, n \times 1$ chloride & 10,14 \\
\hline $75-90-3-\div-\cdots----c h l o r e s$ thene & 10,14 \\
\hline $75-09-2-\cdots-\cdots--$ Methrlene chloride & 10.11 \\
\hline $67-64-1-\cdots-\cdots-$ Acetone & 10,11 \\
\hline $75-15-0-\cdots-\cdots--c_{1}$ rben OLsulfide & 10,11 \\
\hline $75-35-4 \cdots-\cdots-1,1-0$, chloreethene & 10.11 \\
\hline $75-34-3-\cdots \cdots--1,1-0$ ichleroethene & 10,12 \\
\hline $540-59-9-\cdots \cdots--12-01$ chlores thene (totel) & 10.14 \\
\hline $67-66-3-\cdots-\cdots--$ chloroform & $10,1 \overline{1}$ \\
\hline $107-06-2-\cdots---1,2-0, c h$ loreethane & 10,14 \\
\hline $78-93-3-\cdots-\cdots--2-8 u t a n o n e$ & 10.14 \\
\hline $71-55-6 \cdots-\cdots--1,1,1-$ Ir ichloreethone. & 10.112 \\
\hline $56-23-5-\cdots-----$ Carben Ietrochloride & 10.11 \\
\hline 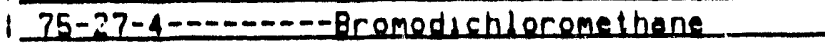 & 10.11 \\
\hline $78-87-5-\cdots-\cdots-1,2-0$ ichloreoreoene & 10.12 \\
\hline $10061-01-5-----c 1 s-13$-01ahlorooronene & 10,12 \\
\hline $79-91-6-\cdots-----$ Irichloresthene & 10.11 \\
\hline $124-48-1-\cdots-\cdots-0$ deremachloromethene & 10.14 \\
\hline $79-90-5-\cdots-\cdots-1,2,2-$ Ir ichlor eathene & 10,12 \\
\hline $71-13-2-\cdots-\cdots--$ Benzene & 10.11 \\
\hline $10961-92-6-\cdots---1$ rans -1 , 3-0ichlereorooene & 10.11 \\
\hline $35-25-2-\cdots-\cdots--8$ romoform & 10,11 \\
\hline $108-10-1-\cdots-\cdots-4-$ Methyl-2-Pentanone & 10,12 \\
\hline $591-78-6 \cdots---\cdots-2-$ Hexenene & 10,14 \\
\hline $127-18-4-\ldots-\cdots-$ Ietrechloreethene & 10.14 \\
\hline $79-34-5-\cdots-\cdots---1,1,2,2$-Ietrachloroetha & $10+14$ \\
\hline $108-88-3-\cdots--\cdots$ Ioluene & 10.14 \\
\hline $108-90-7-\cdots-\cdots-$ Chlorobenzene & 10.12 \\
\hline $19 \theta-41-4-\cdots----$ Ethylbenzene & 10,12 \\
\hline $199-42-5 \cdots-\cdots--5$ y yrene & 10.12 \\
\hline $1330-20-7-\cdots---x \times$ lene (total) & 10.14 \\
\hline
\end{tabular}




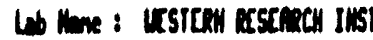

Cave No : will

crenuits mulysis airh sure]

1 suple muber 1

1 aritik

(Poe 2)

Sentuolatile Compounds

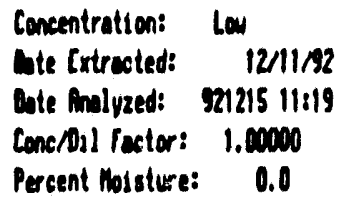

Cet Cleanes - Yes No Mo

Seneralery fumel Extraction _. Yes

Continuovi Liquid-Liquid Extraction $X$ Yes

C.A.S.

$16 / 1$

C.A.S.

Number

Number

$15 / 1$

108-95-2. Phenol

$111-41-4$ bis(2-Chloroethyl)ether

\$5-57-8 2-Chlorophenol

541-73-1 1,3-0ichlerobenzene

106-46-7 1,1-01chlorabenzene

95-50-1 1,2-0ichlorobenzene

95-48-7 2 tiethylphenol

108-60-1 2,2'-oxybis(1-Chloropropene,

106-44-5 4tiethylphenol

621-64-7 N-Hitroso-di-n-orawlenine

67-72-1 Hexachlor oethane

90-95-3 Mitrobenzent

78-59-1 Isophorone

88-75-5 2-Nitrophenol

105-67-9 2,4-Dinethylphenol

$111-91-1$ bis(2-Chlor oethoxy)methane

120-83-2 2,4-Dichlorophenol

120-82-1 1,2,4-Irichlerobenzene

91-20-3 Maphthalene

106-47-8 4-Chloroeniline

07-68-3 Hexachiorobutadiene

59-50-7 4-Chloro-3 methylphenol

91-57-6 2+tethyl mephthalene

7-17-4 Mexchlorecyciopentadiene

80-16-2 2,4,6-Irichleraphenol

s5-5, 2,4,5-Irichlorapheno!

91-58-7 2-Chlor onehthalene

$08-74-12-1$ istromiline

|31-11-3 Dinethylohthulate

209-86-8 Acemphthylene

99-09-2 3-Hitroaniline

03-32-9 Acenuphthene

$\begin{array}{ll}10 & U \\ 10 & U \\ 10 & U \\ 10 & U \\ 10 & U \\ 10 & U \\ 10 & U \\ 10 & U \\ 10 & U \\ 10 & U \\ 10 & U \\ 10 & U \\ 10 & U \\ 10 & U \\ 10 & U \\ 10 & U \\ 10 & U \\ 10 & U \\ 10 & U \\ 10 & U \\ 10 & U \\ 10 & U \\ 10 & U \\ 10 & U \\ 10 & U \\ 25 & U \\ 10 & U \\ 25 & U \\ 10 & U \\ 10 & U \\ 25 & U \\ 10 & U\end{array}$

51-28-5 2,4-Dinitrophenol

100-02-7 4-Hitrophenol

132-69-9 Dibenzofuran

$121-14-2 \quad 2,4-0$ initrotoluene

606-20-2 2,6-0initrotoluene

04-66-2 Diethylphthilate

7005-72-3 4-Chlor ophenyl-phenylether

86-73-7 Pluarene

100-01-6 1-Hitrooniline

534-52-1 1,6-0ini tro-2-nethyiphenol

06-30-6 $N$-Hit trosodiphenylemine (1)

101-55-3 1-Ar mophenyl-phenylether

118-74-1 Nexachlorobenzene

87-06-5 Pentachlorophenol

05-01-8 Phenanthrene

120-12-7 Anthracene

66-74-8 Corbuzole

84-74-2 Bi-n-8utylphtholate

$206-44-00$ rluoranthene

129-00-00 Pyrene

05-68-7 Dulylbenzylahthalote

91-94-1 3,3'-Dichlorobenzidine

56-55-3 Benzo(a) Anthracene

$117-81-7$ bis(2-\{thylhexyl) phthalate

218-01-9 Chrysene

$117-04-00$ Di-n-octulphthalate

205-99-2 Benzo(b) fluoranthene

207-00-9 Benzo(k) fluor on thene

50-32-8 Benzola)Pyrene

193-39-5 Indeno(1,2,3-cd)Purene

53-70-3 Oibenz(a, h) enthracene

191-24-2 benzo $(g, h, j)$ perylene

(1)-Connot be separated from diphenylonine

forn 1 


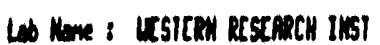

case Ho: nit

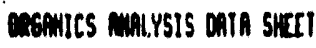

(Pex 2)

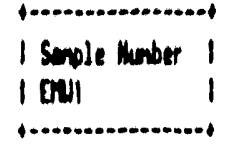

\section{Senivolatile Compounds}

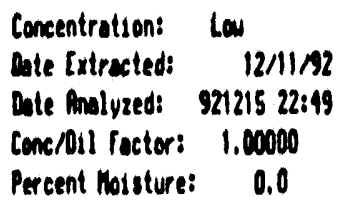

6PC Clames - Yes WX No

Sepuratory fumel cutraction - Yes Contimous Liquid-Liquid cxtraction $Y$ Yes
C.A.S.

Muber

$15 / 1$

\begin{tabular}{|c|c|c|}
\hline $100-95-2$ & Phenol & 10 \\
\hline $111-44-4$ & bis (2-Chloroethyl)ether & 10 \\
\hline $95-57-8$ & 2-Chlor ophenol & 10 \\
\hline $541-73-1$ & 1,3-0ichlorobenzene & 10 \\
\hline $106-46-7$ & 1.4-Dichlorobenzene & 10 \\
\hline $95-50-1$ & 1,2-0ichlorobenzene & 10 \\
\hline $95-18-?$ & 2 thethylphenol & 10 \\
\hline $108-60-1$ & 2.2'-oxybis (1-Chloropropene) & 10 \\
\hline $106-14-5$ & 4thethylotienol & 10 \\
\hline $621-64-7$ & N-Witroso-di-n-propylamine & 10 \\
\hline $67-72-1$ & Mexachlor oe thane & 10 \\
\hline $98 \cdot 95-3$ & Matrobenzene & 10 \\
\hline $78-59-1$ & Isophor one & 10 \\
\hline $88-75-5$ & 2-Hatrophenol & 10 \\
\hline $105-67-9$ & 2,4-Dinethyl phenol & 10 \\
\hline$|11-9|-1$ & bis (2-Chlor oe thoxy) me thane & 10 \\
\hline $129-83-2$ & 2,4-Uichlor ophenol & 10 \\
\hline $120-12-1$ & 1,2,4-Irichlorobenzene & 10 \\
\hline $91 \div \div 0-3$ & Maphthalene & 10 \\
\hline $106-17-8$ & 4-Chlorcansline & 10 \\
\hline $87-68-3$ & Hexachlor obutadiene & 10 \\
\hline $59-50-7$ & 4-Chloro-3-nethylphenol & 10 \\
\hline $91-57-6$ & 2thethylnephthalene & 10 \\
\hline $77-17-4$ & Maxchlor ocvelopentadiene & 10 \\
\hline $80-06-2$ & $2,4,6-$ Irichlorophenol & 10 \\
\hline $95-95-4$ & 2,1,5-Irichlor ophenol & 25 \\
\hline $91-58-7$ & 2-Ehloronaphthalene & 10 \\
\hline $80-74-4$ & $2 \cdot$ Witrouniline & 25 \\
\hline$|3|-||-3$ & Dinethylphthalote & 10 \\
\hline $200-96-8$ & Remaphthylene & 10 \\
\hline $99-09-2$ & 3-W!'ramiline & 25 \\
\hline $83-32-9$ & Acenophthene & 10 \\
\hline
\end{tabular}

C.A.S.

Nunber

$15 / L$

\begin{tabular}{|c|c|c|}
\hline $51-28-5$ & 2,4-0init tr ophenol & 25 \\
\hline $100-02-7$ & 4-Hstrophenol & 25 \\
\hline $132-64-9$ & Dibenzofuran & 10 \\
\hline $121 \cdot 14 \cdot 2$ & $2,4-0$ ini trotoluene & 10 \\
\hline $606-20-2$ & $2,6-0$ ins trololuene & 10 \\
\hline $84-66-2$ & Diethylphthalate & 10 \\
\hline $7005-72-3$ & 4-Chlor ophenyl-phenylether & 10 \\
\hline $86-73-?$ & Huerene & 10 \\
\hline $100-01-6$ & 4- Hitroansline & 25 \\
\hline $534-52-1$ & 1,6-Dinitro-2-nethylphenol & 25 \\
\hline $86-30-6$ & H-Hi trosodiphenylamine (I) & 10 \\
\hline $101-55-3$ & 4-Bromopienyl-phenylether & 10 \\
\hline $118-74-1$ & Hexachlorobenzene & 10 \\
\hline $87-86-5$ & Pentachlor ophenol & 25 \\
\hline $85-01-8$ & Phenanthrene & 10 \\
\hline $120-12-7$ & Anthracene & 10 \\
\hline $86-74-8$ & Carbazole & 10 \\
\hline $84-74-2$ & $D_{2}-n-$ - -utylphthalate & 1 \\
\hline $206-44-00$ & fluoranthene & 10 \\
\hline $129-00-00$ & Pyrene & 10 \\
\hline $85-68-?$ & Butylbenzylphtholate & 10 \\
\hline $91-94-1$ & 3,3'-Dachlor abenzadine & 10 \\
\hline $56-55-3$ & Benzo(a) Anthracene & 10 \\
\hline $117-81-7$ & bis $(z-[t$ thylhexyl) phthalate & 1 \\
\hline $218-01-9$ & Chrysene & 10 \\
\hline $117-84-00$ & $D_{2}-n$-octulphtholote & 10 \\
\hline $205-99-2$ & Benzo(b)fluor anthene & 10 \\
\hline $207-09-9$ & Benzo(h) fluoranthene & 10 \\
\hline $50-32-8$ & Benzola) Pyrene & 10 \\
\hline $193-39-5$ & Indeno $(1,2,3-c d)$ Pyr ene & 10 \\
\hline $53-70-3$ & Dibenz (a, h)anthracene & 10 \\
\hline $191-24-2$ & Benzo(g,h,i)perylene & 10 \\
\hline
\end{tabular}

Torn 1 $7 / 85$ 


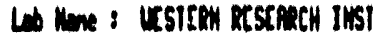

case the : vil

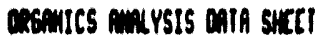

(Page 2)

Senivolatsle Compounds
| Sumple nuber 1 I Cul?
CPC Clamp - Yes Wh no

Sepuratory fumel Extraction - Yes

continuous Liquid-Liquid Extraction $X$ Yes

921215 23:30

concosil fector: 1.00000

Percent Molature: $\quad 0.0$

C.A.S.

Nunber

$16 / 1$

100-95-2 Phenol

111-44-4 bis(2-Chloroethyl)ether

95-57-8 2-Chlor ophenol

511-73-1 1,3-Dichlorobenzene

106-16-7 1,4-0ichlorobenzene

95-50-1 1,2-01chlorobenzene

95-18.7 2thethylphenol

108-60-1 2,2'-oxpbis(1-Chloropr opane)

106-44-5 4thethylphenol

621-64-7 W-Nitroso-di-n-propylemine

67-72-1 Hexochloroethane

98-95-3 Mitrobenzene

78-59-1 isophorone

88-75-5 2-Nitrophenol

105-67-9 2.9-02nethylphenol

$1 i 1-91-1$ bis(2-Chloroethoxy) methane

120-83-2 2,4-Dichlorophenol

120-02-1 1,2,4-Irichlorobenzene

91-20-3 Mephthalene

106-47-8 4-Chloroanjlime

87-68-3 Hexachlorobutadiene

59-50-7 4-Chloro-3 methylphenol

91-57-6 2thethylnophthalene

77-17-4 Hexachlor ocvelopentadiene

88-06-2 2,1,6-Irichlor ophenol

95-95-4 2,4,5-Irichlorophenol

91-58-7. 2-Chlor onsophthalene

88-74-4 2-Hitroanlline

131-11-3 Dinethylphthalote

200-\% -8 Acensphthylene

99-09-2 3-"litraaniline

83-32-9 Acenaphthene
C.A.S.

Munber

W/L

51-28-5 2,4-0initropheno!

$25 \mathrm{~V}$

100-02-7 4- Hitrophenol

132-64-9 Dibenzoluran

$25 \mathrm{U}$

$10 \mathrm{U}$

$10 \mathrm{~V}$

$10 \mathrm{~V}$

121-14-2 2,4-01nitrotoluene

606-20-2 2,6-Dins trotoluene

84-66.2 Orethylphtholate

7005-?2-3 4-Chlorophenyl-otienylether

86-73.7 Tluorene

100-01-6 4-Hitrasniline

534-52-1 9,6-0.ns tro-2-nethylphenol

86-30-6 N-Mitrosodiphenylenine (1)

101-55-3 1-8remophenyl-phenylether

118-74-1 Hexachlor obenzene

87-86-5 Pentachloraphenol

85-01-8 Phenanthrene

120-12-? Anthracene

06-74-8 Corbozole

84-74-2 01-n-Butylphthalale

206-44-00 Fluoranthene

129-00-00 Pyrene

B5-68-7 Cutylbeizulohthalate

$91-94-1 \quad 3,3^{\prime} \cdot$ Dichlopobenzadine

56-55-3 cenzo(a) Anthracene

$117-81 \cdot 7$ bis(2-[thylhexyl)phthalate

218-01-9 Chrysene

117-84-00 Di-n-octylphthalate

205-99-2 Beri2o(b) fluoranthene

207-08-9 Benzo(k)fluor anthene

50-32-8 Benzola)Purcine

193-39-5 Indeno(1,2,3-cd)Pyrene

53-70-3 Dabenz(a, h)anthracene

191-24-2 Benzo(g,h, l)perylene

(1)-Cannot be separated fron diphenylarine 
Lot lime : Irstcen Resrenty IIST

case 10 : vil

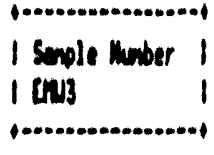

Sentuolatile lempoundo

Cot Clemue _ Yes No no Sepersolory furmal Extruction - Ves

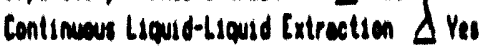

C.A.5.

Number

Muber

1012

-

51-28-5 2,4-0instropheno!

100-02-9 4-Hitrophenol

132.64-9 Dibenzofuran

$121 \cdot 14 \cdot 2 \quad 2,4 \cdot 0$ ins trotoluene

$606-20-2 \quad 2,6-0$ initrotoluene

84-66-2 Diethyl phthalole

7005-72-3 4-Chlocophenyl-ohenylet ther

66-73.7 Fluor ene

100-01-6 4-Whitrouniline

534-52-1 $\quad$ 1,6-01ns tro-2-nethylphenol

06-30-6 M-Mitrosodiphenyleinine (1)

101-55-3 4-Bronophenyl-ohenyle ther

$118-24-1$ Hewachlorobenzene

87-86-5 Pentachloropheno]

B5-01-8 Phenenthrene

120-12.7 inthracene

86-74.8 Corbozole

04-74-2 01-n-butylphtholote

206-44-00 rluorenthene

129-00-00 Pyrene

05-66-7 Cutylbenzylohthalate

$91-94-1 \quad 3,3^{\prime} \cdot$ - Dichlorobenzidsne

56-55-3 Benzo(6) Anthrocene

$117 \cdot 01 \cdot 9$ Bro(2-[thylhexyl) ahthalate

218-01-9 Chryoere

117-04-00 0 02 - n-octylphthalate

205-99-2 Benzo(b) fllworenthene

207-00-9 Eenzo(k) Illuorenthene

50-32-6 Benzolo)Pyene

193-39-5 Indeno(1,2,3-ed)Pyrene

53-70-3 Dabenz(a, himentrocene

191-24-? Benzo(g,h,i)perylene

(1)- Comot be separated from diphenylasune

$99 \cdot 09-2 \cdot 3 \cdot$ lititrantlis $x$ U

$25 \mathrm{~V}$

$10 \mathrm{U}$

$10 \mathrm{U}$

$10 \mathrm{~V}$

$10 \mathrm{U}$

$10 \mathrm{~V}$

$10 \mathrm{~V}$

$25 \mathrm{~V}$

$25 \mathrm{~V}$

$10 \mathrm{U}$

$10 \mathrm{U}$

$10 \mathrm{~V}$

$25 \mathrm{~V}$

$10 \mathrm{U}$

$10 \mathrm{~V}$

$10 \mathrm{~V}$

$1 \mathrm{~J}$

$10 \mathrm{~V}$

$10 \mathrm{U}$

$10 \mathrm{~V}$

$10 \mathrm{U}$

$10 \mathrm{~V}$

21

$10 \mathrm{~V}$

$10 \mathrm{~V}$

$10 \mathrm{~V}$

$10 \mathrm{~V}$

$10 \mathrm{~V}$

$10 \mathrm{~V}$

$10 \mathrm{~V}$

$10 \mathrm{~V}$ 


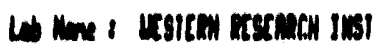
cane $N_{0} 1$ and

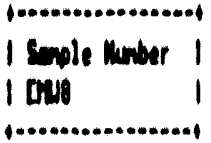

connites mursts bain sietl

(Page 2)

Senivolatile compounds

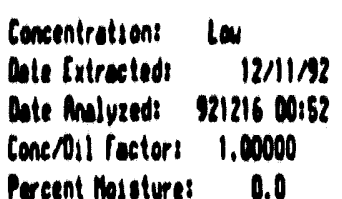

C.A.S.

Nunber

1009-95.2 Phonol

$111 \cdot 44-4$ bis(2-Chloroethyl)ether

\$5.57-0 2-Chlorophenol

511-73.1 1,3-01chlorobenzene

$106-46-91,4 \cdot 01$ chlor obenzene

55-50-1 1,2-0ichlorobenzene

55. $10 . ?$ 2 the thylohenol

108-60-1 2,2'-axybio(1-Chloropropenne)

106-41-5 1thethylphenol

$621 \cdot 64-?$ Hellitroso-dd -n propularine

$67.72-1$ Hexachloroethane

98-95-3 Mitrobenzene

78-59.1 Isophorone

80-75-5 2- Hitrophenol

105-67-9 2,4-Dinethylohenol

$111-91-1$ bie(2-Chloroethoxy)methune

120-83.2 2,4-Diehlor ophenol

$120-82-1 \quad 1,2,4-$ Iriehlordenzene

91-20-3 Mophtholene

$106-47-8$ 4-Chleronilline

$07-68-3$ Hexchlordoutadiene

59-50-7 +Chloro-3 methylohenol

91-57-6 2tizthylnemithalene

n-47-4 Hrachlorecyolopentediene

06-06-2 2,4,6-Irichlerashenol

\$5-\$5-4 2,4,5-Irichlerophenol

$91-50.72 \cdot$ Chloronehthulene

08-74-4 2-Nitrandline

$131-11-3$ Ainethylphthalate

200-\%-8 Mcemohthylene

99-09-2 3-Hitrouniline

03-32-9 Renaphthene

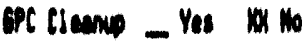 \\ Sepoutory fumel trtraction - Yes \\ centinuous Liqud-Liquid Citruction $\mathrm{X}$ Yes
}

C.A.S.

Henber

$16 / 1$

51-20-5 2,4-01nitrophenol

$25 \mathrm{~V}$

100-02-9 4-Hetrophenol

132.64.9 Dibenzofuran

$25 \mathrm{U}$

121.-64.

$606-20 \cdot 2,2,6-0$ inst trotoluene

$10 \mathrm{~V}$

$10 \mathrm{~V}$

$10 \mathrm{~V}$

$10 \mathrm{~V}$

$10 \mathrm{~V}$

10

$10 \mathrm{~V}$

$10 \mathrm{~V}$

$10 \mathrm{~V}$

$10 \mathrm{~V}$

$10 \mathrm{~V}$

$10 \mathrm{~V}$

$10 \mathrm{~V}$

$10 \mathrm{~V}$

$10 \mathrm{U}$

$10 \mathrm{~V}$

$10 \mathrm{~V}$

$10 \mathrm{~V}$

$10 \mathrm{~V}$

$10 \mathrm{~V}$

$10 \mathrm{~V}$

$10 \mathrm{~V}$

$10 \mathrm{~V}$

$10 \mathrm{~V}$

20

$10 \mathrm{~V}$

$5 \mathrm{~V}$

$10 \mathrm{~V}$

$10 \mathrm{~V}$

$25 \mathrm{~V}$

$10 \mathrm{~V}$
84-66-2 Diethylphthilale

$7005-72-3 \quad 4-$ Chlor ophenyl - phenylether

86.73-7 fluorene

100-01-6 4-Hitraniline

534-52-1 1,6-01nis tro-2-ne thylphenol

86-30-6 H-tutrosodiphenylerine (1)

101-55-3 4-Eramophenyl-phenyle ther

118-74-1 Mewachlorobenzene

07-86-5 Pentachlorophenol

85-01-8 Phenenthrene

$120 \cdot 12 \cdot 7$ mintracene

06-74-8 Corbuzole

14-74-2 M1-n-whtylphthalote

$206-44-00$ fluerenthene

129-00-00 Purene

65-60-7 cutylbenzylphthalate

$91-99-1$ 3,3'-Oichlorabentidine

B6-55.3 Enzo(a) finthracene

$117 \cdot 01 \cdot 7$ Mas(2-[thylhexyl) phtholate

218-01-9 Chryeere

$117-94-\infty 0$ Di n-actylohtholate

205-99.2 Enzo(b)fluorenthene

207-00-9 enso(k)fluorenthene

50-32-0 emzo(a)Pyrene

193-39-5 Indeno(1,2,3-cd)Purene

53-70-3 Oibenz(a,h)enthracene

191-24-2 Benzo(g,h,j)perylene

(1)-Connot be separated tron diphenylessne 


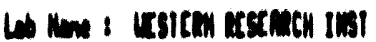

can in : in

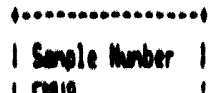

1 oung

menvics marsis onin suct

(ban 2)

Enivolutile Cempands

\begin{abstract}
Comentrutiont lew
Dte trtrcated: 12/11/n?

che mulyzeds $\$ 21216$ 01128

Concoil recters 1.0000

Percent hastures 0.0
\end{abstract}

OC Clanup - Yes W No

encotory funmel cxtrestion - Yes

Continuan Liquid-Liquid fatraction \& Yea
C.A.S.

Muber

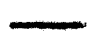

10.95-2 Amonol

111.44 .9 bio(2-Chlerathyl)ether

\$.57-8 2-Chlorophenol

$511 \cdot 73 \cdot 11,3 \cdot 01$ ehlordenzene

$105 \cdot 16 \cdot 7 \quad 1,9-$ Oiehlerobenzene

\$5-50-1 1,2-01chlorebenzene

55.10-1 2t thethyldmenol

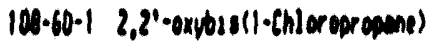

I0s-41-5 ithethylohenol

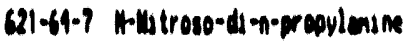

69.72.1 Hexachlocoethene

\%.\$5.3 Mitrobenzeme

$70 \cdot 59-1$ Isepherone

09-75-5 2-litrophenol

105-67-9 2,4-01nethylphenol

$\mid 11 \cdot 91 \cdot 1$ bis(2-Chlor oethoxymethene

[20-83-2 2,4-01chloroshenol

$120-02 \cdot 1 \quad 1,2,4-1$ richlorobenzene

91-20-3 Menthilene

106-17-1 4-thloransiline

87-68-3 Mechlorobuladiene

59-50-? 4-Chlero-3 -nethul phenol

91.57-6 2thethylnophthalene

77-47-1 Menchlorocyelopentediene

80.06-2 2,1,6-Irichlor ophenol

\$5-55-1 2,1,5-1rsichlor ophenol

$91-50-72-$ Onloronephthalen

3-74-4 2-Hitransiline

|31-11-3 Oinethylohthalate

$200 \cdot \%-8$ meenach thylene

y.09-2 J-listraniline

13-32-9 Rernophthene wn

C.M.S.

hriver

161

$51-20 \cdot 52,4 \cdot 0$ ins traphenol is $\mathrm{U}$

100-02-7 4-littrophenol

132-64-9 Dibenzolurm

$121-14-2 \quad 2,4-01$ instrololuene

$606-20 \cdot 2 \quad 2,6-0$ initrotoluen

14.66-2 Diethylphtholote

7005-72-3 4-thlor ophend-ahenylether

06-73-9 Pluorene

100-01-6 4-Hitranilime

534-52-1 4,6-01nitro-2-nethylohenol

86-30-6 N-llitrosodiphenylacine (1)

101-55-3 4-8rapophenyl-phenylether

118-74-1 Mexuchlorobenzene

17-86-5 Pentachlorophenol

85.01.8 Phenenthrene

120-12.9 mithrocent

06-74-8 Corbazole

$04 \cdot 74 \cdot 2$ O1-n-Etulphthalete

$206-14 \cdot 00$ rluaranthene

129-00-00 Pyrene

65-68.7 witylbenzylahthalate

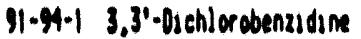

56-55-3 cenzo(a)finthrocene

117-81-7 bis(2-[thylhexyl)onthalate

218-01-8 chrusene

$117-94-00 D_{1}-n \cdot x^{2}$ tylahthalate

205-99-2 Benzo(b)fluoranthene

207-01-9 enzo(h) fluorenthene

So-32-9 Cenzo(a)Pyrene

193-39-5 Indeno(1,2,3-cd) Pureme

53-70-3 Dibena(a,h) monthracene

191-24-2 Benzo(g,h,i)perylene

(1)-Connot be separated Iron diphenyicuine
26

$10 \mathrm{~V}$

$10 \mathrm{U}$

$10 \mathrm{~V}$

$10 \mathrm{~V}$

$10 \mathrm{~V}$

$10 \mathrm{~V}$

$25 \mathrm{~V}$

$25 \mathrm{~V}$

$10 \mathrm{~V}$

$10 \mathrm{~V}$

$10 \mathrm{~V}$

$25 \mathrm{~V}$

$10 \mathrm{~V}$

$10 \mathrm{~V}$

$10 \mathrm{~V}$

$10 \mathrm{U}$

$10 \mathrm{~V}$

$10 \mathrm{U}$

$10 \mathrm{~V}$

$10 \mathrm{~V}$

$10 \mathrm{~V}$

21

$10 \mathrm{~V}$

$10 \mathrm{U}$

$10 \mathrm{~V}$

$10 \mathrm{~V}$

$10 \mathrm{U}$

$10 \mathrm{~V}$

$10 \mathrm{U}$

$10 \mathrm{~V}$ 


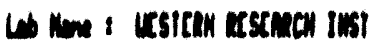

case in : at

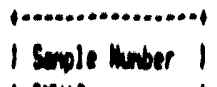

1 (s)lo

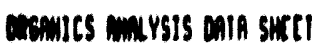

(Pux 2)

Emivolatile cenpounds

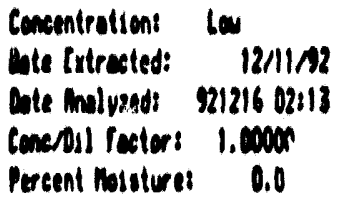

C.A.S.

Muser

100.45.2 Phemel

$111 \cdot 14-4$ bis(2-Chloroethyl) ther

\$5.57.1 2-Ehlorophenol

S11.93.1 1,3-01schlorabenzene

$106-16-9$ 1,4-01chlorobenzene

S5.50-1 1,2-01chlordobenzene

$95-10 \cdot 92$ the thylohenol

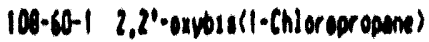

106.41.5 1 thethylphenol

(21.64-9 N-nitroso-d1-n-propulauine

69.92.1 Musthlor oethene

Qx.y5-3 Mitrobenzene

78-59-1 leophorone

M.75-5 2-Wiltrophenol

105.69.9 2.4-0ine thyiphenol

III-9I-1 bus(2-Chloroethoxymethene

120-83-2 2,1901chloropheno!

120-82-1 1,2,4-1richlorobenzene

$\$ 1.20 .3$ mophthalem

106. 17.8 4-Chlorconsline

17968.3 Meractloc obuladiene

59-50-9 4-ChNor 0-3-3ne Inylahenol

91.57 .62 thethylnowhthalene

77.97-1 Muchl or oevelopentediene

80-06-2 2,4,6-Irietlerophenol

55.45-1 2,1,5-Irichlorophenol

$91-58.92$-chloronephthalene

10-74-1 2-Hitromslion

$|3| \cdot \mid 1 \cdot 3$ Oinethylahtholate

200-\%.1 Eemohthyleme

M.09-2 3-Hitraniline

03-32-9 Acenaphtivene
APC Clempo - Yes Ho

Seperotery fumel Extrection - Yes

continwous Liewid-liquid fatrection $\searrow$ Yes

C.A.S.

number

w5/h

$51-28-52,4-0$ snstroohenol

100-02.9 4. Hit trophenol

132-64.9 Dibenzoluren

$121-14 \cdot 2 \quad 2,4-01$ instrotoluene

$606-20.2 \quad 2.6-0$. instrotoluene

04.66.2 Denthylphimelate

7005-22-3 1-Chlorophenyl-ophenylether

66-73-9 Cllworene

$100-01-6$ 4-Hitranilime

$534-52 \cdot 1 \quad$ 1,6-0.nat $10-2$ methylphenol

16-30-6 M-Hitrosodiplenylenine (1)

101-55-3 4- Ar monophenyl-phenylether

$118-74-1$ Herochlordonzene

07.86-5 Pentochlorophenol

85-01.8 Phenenthrene

120-12.9 Anthroceme

36-27.8 Cartazole

B4-74-2 D1-n-Butylahthalale

206 -11. bi fluopenthene

129-00.00 Prene

85-60-9 Dutylbenzylphthalote

$91-94 \cdot 1 \quad 3,31-01$ chlor doenzidine

56-55-3 Benzo(a) minthracene

$117-011 \cdot ?$ bse $(2-2$ thylhexyl)onthalote

210-01-9 Cirysere

$117-04-0000_{1}-n-0$ ectyiphthalote

$205-99 .-2$ Rnzo(b) flluoremithene

207.08.9 Benzo(k)/lluorenthene

50-32.1 Benzo(o) Pyrems.

193-39-5 Indeno(1,2,3-cd)Pyrene

$55-70-3$ Dibenz(a, h) entrocene

191-24-2 Benzo(g, h, diperylene

(1)- Connot be separaled tren diphenyleuse

Porn 1 


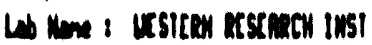

ane to 1 ill

| smole Mublep I

I Dulla

onsomics matysis gain suret

(Pape 2)

Sentwolotile Conpounds

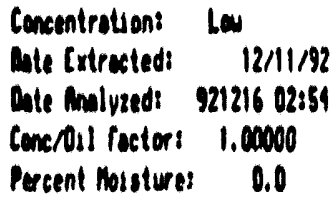

ext Cleanud - Yei $\$$ Ho

Seperotopy fiomel tatraction _ Yes

Continuous liquid-Liquid Cxtrection $\not$ Yes

C.A.S.

C.A.S.

Nurber

U6/L

Munber

1512

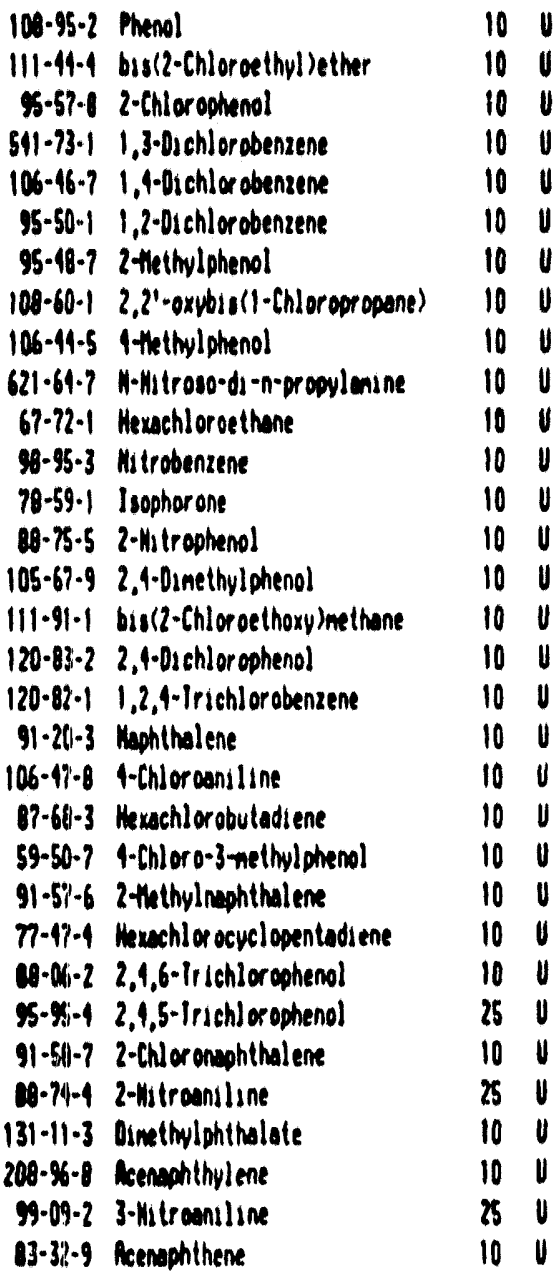

$51-28.52,4-$ Oinitrophenol $25 \mathrm{U}$

100-02-7 4-Hitrophenol $25 \mathrm{U}$

132.64-9 Dibenzoluran $10 \mathrm{~V}$

121-14-2 2,4-Dinitrotoluene $\quad 10 \mathrm{U}$

$606-20-22,6-0$ initrotoluene $10 \mathrm{U}$

84-66-2 Diethylphthalate i0 $\mathrm{U}$

2005-72-3 1-Chlerophenyl-ohenylether $10 \mathrm{~V}$

$16-73-7$ llooreme $10 \mathrm{U}$

$100-01-6$ 1-Hitroaniline $25 \mathrm{U}$

534-52-1 1,6-0instro-2-nethulphenol $25 \mathrm{U}$

6-30-6 H-Hitrosodiphenyleaine (1) $10 \mathrm{~V}$

101-55-3 4-Eromophenyl-phenulether $10 \mathrm{~V}$

118-74-1 Hexuchlorobenzene $10 \mathrm{~V}$

87-86-5 Pentachlor optenol $25 \mathrm{~V}$

05-01-8 Phenenthrene $10 \mathrm{U}$

120-12-7 Anthracene $10 \mathrm{~V}$

06-74-8 Carbazole $10 \mathrm{U}$

94-74-2 4 -n-Butylphthalate $10 \mathrm{U}$

206-14-00 fluorenthene $10 \mathrm{U}$

129-00-00 Purene $10 \mathrm{U}$

85-68-7 Butylbenzulphthalate $10 \mathrm{~V}$

$91-94-1 \quad 3,3^{\prime}-$ Dzchlorobenzidine $\quad 10 \mathrm{~V}$

56.55.3 Cenzo(o) Anthracene $10 \mathrm{U}$

$117-81-7$ bis (2-[thylhexy) phishalate $2 \mathrm{~J}$

218-01-9 Chrusene $10 \mathrm{~V}$

117-84-00 01-n-octylphthalste $\quad 10 \mathrm{U}$

205.99-2 Benzo(b)fliwaranthene is U

207-08-9 Aenzo(k)fluoranthene $10 \mathrm{U}$

50-32-8 Benzo(a)Purene $10 \mathrm{~V}$

193-39-5 Indeno(1,2,3-ed)Purene $10 \mathrm{U}$

53-20-3 Dibenz $(a, h)$ anthracene $10 \mathrm{U}$

$191 \cdot 24-2$ Penzo $(0, h, 1)$ perylene $10 \mathrm{U}$

(1)-Connot be separated fron diphenylanine

Torn 1 
Lob hane : Irsitan Restrech INSI

Case Ho: Fill
Cmecentration: Low Ste Extracted: 12/11/92 Date inalyzed: 921216 03:35 Conc/Oil factor: 1.00000 Percent Moisture: $\quad 0.0$

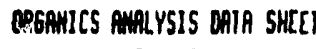
(Page 2)

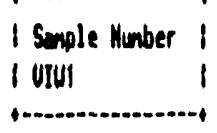

Serivolatile Compsunds
C.A.S.

Nuber

$16 n$

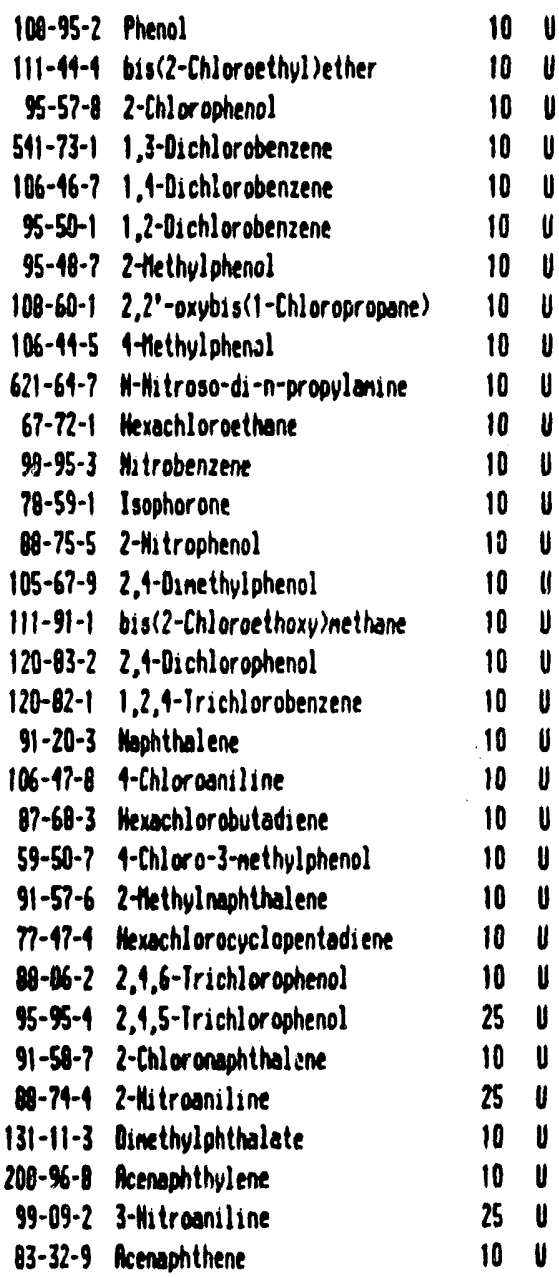

6PC Cleanup - Yes XX Ho

Separatory Tunne? Extraction - Yes Continuous Liquid-Liquid Extraction $X$ Yes
C.A.S.

Number

$16 / L$

51-28-5 2,4-0initrophenol 25

100-02-? 4-Hitrophenol $25 \mathrm{~V}$

132-64-9 Dibenzofuran $10 \mathrm{U}$

121-14-2 2,4-Dinatrotoluene $10 \mathrm{U}$

606-20-2 2,6-Dinitrotoluene $10 \mathrm{U}$

84-66-2 Oiethylphthalate $10 \mathrm{U}$

7005-72-3 9-Chlorophenyl-phenylether $10 \mathrm{~V}$

86-73-7 Hluorene $10 \mathrm{U}$

100-01-6 4-Hitroaniline

534-52-1 4,6-0initro-2-nethylphenol $25 \mathrm{U}$

$86-30-6$ H-Hitrosodiphenylarine (1) $10 \mathrm{U}$

101-55-3 1-Bromophenyl-phenylether $10 \mathrm{~V}$

118-74-1 Hexachlorobenzene $10 \mathrm{U}$

87-86-5 Pentachlorophenol $25 \mathrm{U}$

85-01-8 Phenanthrene $10 \mathrm{U}$

120-12-7 Anthracene $10 \mathrm{U}$

86-74-8 Carbazole $10 \mathrm{U}$

84-74-2 B1-n-8utylphtholate $\quad 1 \mathrm{~J}$

206-44-00 Iluorantherie $\quad 10 \mathrm{U}$

129-00-00 Pyrene $10 \mathrm{U}$

85-68-7 Butylbenzylphthalate $10 \mathrm{~V}$

$91-94-1 \quad 3,3^{\circ}$-Dichlorobenzidine $\quad 10 \mathrm{U}$

56-55-3 benzo(a)Ainthracene 10 U

$117-81-7$ bis(2-Ethylhexyl)phtholate $4 \mathrm{~J}$

218-01-9 Chrysene $10 \mathrm{U}$

117-84-00 Di-n-octylphthalate $10 \mathrm{U}$

205-99-2 Benzo(b)fluoranthene $10 \mathrm{U}$

207-08-9 Benzo(k)fluoranthene $10 \mathrm{U}$

50-32-8 Benzo(a)Pyrene $10 \mathrm{U}$

193-39-5 Indeno(1,2,3-cd)Purene $10 \mathrm{U}$

53-70-3 Dibenz(a,h)anthracene $10 \mathrm{U}$

191-24-2 Benzo(g,h,i)pervlene $10 \mathrm{U}$

(1)-Cannot be separated fron diphenylamine 


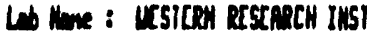

case No : cononics maiysts oarh suter

(Poge 2)

$\begin{array}{ll}\text { Concentration: } & \text { Low } \\ \text { Cote [xtracted: } & 12 / 11 / 92 \\ \text { Cule Praluzed: } & 22121609: 15 \\ \text { Conc/Oil roctor: } & 1.00000 \\ \text { Percent Moisture: } & 0.0\end{array}$

Senivolotile Compounds

SPC Cleanup - Yes WK Ho

Separatory funnel Ixtraction - Yes

Coritinuous Liquid-liquid Extraction $\&$ Yes

C.A.S.

Muber

$16 / 1$

C.A.S.

Nunber

$16 /$

108-95-2 Phenol

1I1-44-4 bis(2-Chloroethyl)ether

\$5-57-8 2-Chlorophenol

$511-73-1$ 1,3-01chlorobenzene

106-46-7 1,4-Dichlor obenzene

95-50-1 1,2-Dichlorobenzene

95-18-? 2+thethulphenol

108-60-1 2,2'-oxpbis(1-Chlor opropene)

106-44-5 t thethylatenol

621-64-7 H-Hitroso-dz-n-propyleaine

67-72-1 Hewachloroe thane

98-95-3 Mitrobenzene

78-59-1 Iscophor one

08-75-5 2-Hitrophenol

105-67-9 2,4-0ine thylphenol

$111-91-1$ bis(2-Chlor oethoxy) methane

120-83-2 2,4-0ichlor ophenol

120-82-1 1,2,4-Iruchlorobenzene

91-20-3 Maphthalene

106-47-8 4-Chloraniline

87-68-3 Hexachlorobutadiene

59-50-7 4-Chloro-3-nethylahenol

91-57-6 2thethylnophthalene

77-47-4 Hemachloracuclosentodiene

80-06-2 2,1,6-Irichlorophenol

95-\%5-4 2,1,5-Irichlorophenol

91-50-7 2-Chloromaphthalene

88-74-4 2-Hitraniline

|3|-11-3 Dinethylphtholate

209-\%-8 Acenomithylene

99-09-2 3-listrouniline

83-32-9 Menaph!hene

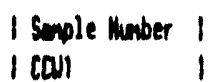

( cal

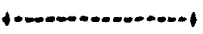

\section{.}

104

$10 \mathrm{~V}$

$10 \mathrm{~V}$

$10 \mathrm{U}$

$10 \mathrm{U}$

$10 \mathrm{U}$

$10 \mathrm{~V}$

$10 \mathrm{~V}$

$10 \mathrm{~V}$

$10 \mathrm{~V}$

$10 \mathrm{~V}$

$10 \mathrm{~V}$

$10 \mathrm{U}$

$10 \mathrm{~V}$

$10 \mathrm{U}$

$10 \mathrm{~V}$

$10 \mathrm{~V}$

$10 \mathrm{U}$

$10 \mathrm{~V}$

100

$10 \mathrm{~V}$

$10 \mathrm{~V}$

$10 \mathrm{~V}$

$10 \mathrm{~V}$

$10 \mathrm{~V}$

$25 \mathrm{~V}$

$10 \mathrm{U}$

$25 \mathrm{~V}$

$10 \mathrm{~V}$

$10 \mathrm{~V}$

254

$10 \mathrm{U}$
51-28-5 2,4-0initrophenol

100-02-7 4-Kitrophenol

132-64-9 Dibenzofuran

121-14-2 2,4-Dinitrotoluene

606-20-2 2,6-Dinitrotoluene

84-66-2 Diethylphthalate

2005-72-3 1-Chlor ophenyl-phenylether

06-73-7 fluorene

100-01-6 4-Hitroaniline

534-52-1 4,6-0initro-2 ne thyl phenol

06-30-6 H-Hitrosodiphenylamine (1)

101-55-3 4-Eromophenyl-phenylether

118-74-1 Hexachlor obenzene

07-86-5 Pentachlor ophenol

85-01-8 Phenanthrene

120-12-7 Anthracene

86-74-8 Carbazole

B4-74-2 Di-n-Butylahthalate

206-44-00 Fluoranthene

129-00-00 Purene

85-68-7 Dutylbenzylohthalate

91-94-1 3,3'-Dichlorabenzidine

56-55-3 Cenzois) finthracene

117-81-7 bis(2-[thylhexyl)phthalate

218-01-9 Chrysene

117-84-00 01-n-octylohthalate

205-99-2 Benzo(b) fllueranthene

207-08-9 Cenzo(k)fluoronthene

50-32-8 Beicicola)Purene

193-39-5 Indeno(1,2,3-cd)Pyrene

53-20-3 Oibenz(o, h) entwracene

191-24-2 Benzo(g,h, 1 )perylene
$25 \mathrm{~V}$

$25 \mathrm{~V}$

$10 \mathrm{~V}$

$10 \mathrm{~V}$

$10 \mathrm{~V}$

$10 \mathrm{~V}$

$10 \mathrm{~V}$

$10 \mathrm{U}$

250

$25 \mathrm{~V}$

$10 \mathrm{~V}$

$10 \mathrm{~V}$

$10 \mathrm{~V}$

$25 \mathrm{U}$

$10 \mathrm{U}$

$10 \mathrm{~V}$

$10 \mathrm{U}$

$1 \mathrm{~J}$

$10 \mathrm{~V}$

$10 \mathrm{~V}$

$10 \mathrm{~V}$

$10 \mathrm{~V}$

$10 \mathrm{~V}$

$2 \mathrm{~J}$

$10 \mathrm{~V}$

$10 \mathrm{~V}$

$10 \mathrm{~V}$

$10 \mathrm{~V}$

$10 \mathrm{~V}$

$10 \mathrm{~V}$

$10 \mathrm{~V}$

$10 \mathrm{~V}$

(1)-Comot be separated fran diphenyleasne 


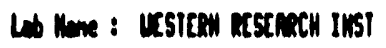
Can No: nit

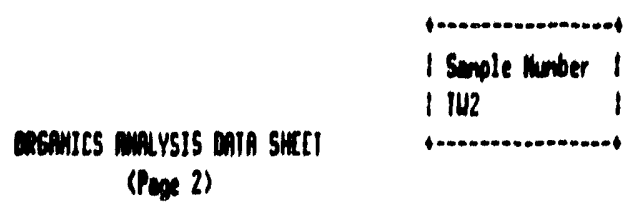

Sentuoletile Compounds

opC Cleanip - Yes No no

Seperotory fumel crtrection - Yes

Continuous Liquid-Liquid Extrection $\chi$ Yes

C.A.S.

number $\quad V_{0} / \mathrm{L}$

$15 n$

51-28-5 2,4-Dins trophenol

100-02-9 4-Hitraphenol

132-64-9 Dibenzofuren

121-14-2 2,4-01nitrololuene

606-20-2 2,6-0initrotoluene

84-66-2 Diethyl phthalate

7005-72-3 4-Chlorophenyl-phenyle ther

86-73-7 fluorene

100-01-6 4-Hitroaniline

534-52-1 1,6-02nitro-2nethylphenol

86-30-6 M-Hitrosodiphenulenine (1)

101-55-3 4-Bromophenyl-phenyle ther

118-74-1 Hexochlor obenzene

17-86-5 Pentachlorophenol

85-01-8 Phenanthrene

120-12-7 mithracene

46-79-8 Carbazole

04-74-2 Di-n-tutylohthalate

206-14-00 Pluorenthene

129-00-00 Purene

B5-68-9 Dutylbenzyl Dhthalote

91-94-1 3,3'-Olchlorobenzidine

56-55-3 Benzo(o) An throcene

117-81-7 bis(2-[thylhexyl) phthalate

218-01-9 Cirrycene

$117-04-00$ Oi-n-octylonthalate

205-99-2 Renzo(b) flluorenthene

207-009-9 Ienzo(k) fluorenthene

50-32-8 Renzo(a) Pyrene

193-39-5 Indeno(1,2,3-cd)Pvrene

53-70-3 Dibenz(6, h) onthracene

191-24-2 Benzo(g,h,i)perylene

(1)-Comot be separated Iron diphenylenine

$\begin{array}{cc}25 & U \\ 25 & U \\ 10 & U \\ 10 & U \\ 10 & U \\ 10 & U \\ 10 & U \\ 10 & U \\ 25 & U \\ 25 & U \\ 10 & U \\ 10 & U \\ 10 & U \\ 25 & U \\ 10 & U \\ 10 & U \\ 10 & U \\ 1 & J \\ 10 & U \\ 10 & U \\ 10 & U \\ 10 & U \\ 10 & U \\ 1 & J \\ 10 & U \\ 10 & U \\ 10 & U \\ 10 & U \\ 10 & U \\ 10 & U \\ 10 & U \\ 10 & U\end{array}$

forn 1 


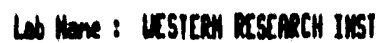

Cose no : Ell

.

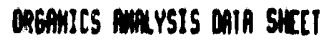

1 smole Maber 1

I I 143

(Poge 2)

Senivolatile Compounds

CPC Cleanup - Yes WN Ho

Seperatory funnel Extraction - Yes Continuous Liquid-Liquid Extraction \& Yes
12/11/92

Conc/Oil fector: 1.00000

Percent hoisture: 0.0

\section{C.R.S.}

Munber
161

108-95-2 Thenol

$111-44-4$ bis(2-Chloroethyl)ether

55-57-82-Chlorophenol

511-73-1 1,3-0ichlorobenzene

106-46-7 1,1-0ichlorobenzene

95-50-1 1,2-0ichlorobenzene

95-48-7 2thethylphenol

100-60-1 2,2'-oxybis(1-Chior opropene)

106-14-5 1tikthylohenol

$621-64-7$ H-Nitroso-di-n-provilenine

67-72-1 Hersechloroethane

98-95-3 Mitrobenzene

78-59-1 Isophor one

18-75-5 2-Hitrophenol

105-67-9 2,4-Dime thylphenol

$111-91-1$ bis(2-Chlor oethoxy) methene

120-83-2 2,4-0ichlor aphenol

120-82-1 1,2,4-Irichlorobenzene

91-20-3 Monthalene

106-19-8 4-Chloraeniline

87-68-3 Mexachlorobutediene

59-50-7 4-Chioro-3-nethylahenol

91-57-6 2+tiethylnophthalene

$77-17-4$ Hexuchlor ocyclopentediene

88-06-2 2,1,6-Irichlorophenol

\$5-55-4 2,4,5-1 richloraphenol

91-58-7 2-Chloronaphthalene

86-74-4 2-Hitrouniline

$131-11-3$ Dinethyl phthalate

200-88-8 Acenaphtthylene

99-09-2 3-Witraaniline

83-32-9 Acenephthene

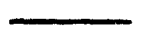

$\begin{array}{ll}10 & U \\ 10 & U \\ 10 & U \\ 10 & U \\ 10 & U \\ 10 & U \\ 10 & U \\ 10 & U \\ 10 & U \\ 10 & U \\ 10 & U \\ 10 & U \\ 10 & U \\ 10 & U \\ 10 & U \\ 10 & U \\ 10 & U \\ 10 & U \\ 10 & U \\ 10 & U \\ 10 & U \\ 10 & U \\ 10 & U \\ 10 & U \\ 10 & U \\ 25 & U \\ 10 & U \\ 25 & U \\ 10 & U \\ 10 & U \\ 25 & U \\ 10 & U\end{array}$

C.A.S.

Nunber

$16 / 1$

51-28-5 2,4-Dinitrophenol 25

100-02-7 4-Nitrophenol $25 \mathrm{U}$

132-69-9 Dibenzoluron $10 \mathrm{U}$

121-14-2 2,4-0instrotoluene $\quad 10 \mathrm{U}$

606-20-2 2,6-0initrotoluene $10 \mathrm{~V}$

84-66-2 Diethylphthalate $10 \mathrm{U}$

7005-72-3 1-Chlorophenyl-phenylether $10 \mathrm{U}$

06-73-7 Fluorene $10 \mathrm{U}$

100-01-6 4-Nistroansline $25 \mathrm{U}$

534-52-1 1,6-0initro-2-nethylphenol $25 \mathrm{U}$

66-30-6 H-Hitrosodiphenylemine (1) $10 \mathrm{U}$

101-55-3 4-Eromophenyl-phenylether $10 \mathrm{~V}$

118-74-1 Hexachlorobenzene $10 \mathrm{U}$

87-86-5 Pentachloropher.01 $25 \mathrm{U}$

85-01-8 Phenanthrene $10 \mathrm{U}$

120-12-7 Anthracene 10 $\mathrm{U}$

86-74-8 Carbozole $10 \mathrm{~V}$

B4-74-2 Di-n-Dutylphthalate $1 \mathrm{~J}$

206-44-00 Pluorenthene $10 \mathrm{~V}$

129-00-00 Pyrene $10 \mathrm{U}$

B5-68-7 Qutylbenzylphthalate $10 \mathrm{U}$

91-94-1 3,3'-Dichlorobenzidine $\quad 10 \mathrm{~V}$

56-55-3 Benzo(a) inthracene $10 \mathrm{U}$

$117-81-7$ bis(2-[thylhexyl) phthalate $1 \mathrm{~J}$

218-01-9 Chrysene $10 \mathrm{U}$

117-84-00 Bi-n-actylphthalate $10 \mathrm{U}$

205-99-2 Benzo(b)fluoranthene $10 \mathrm{U}$

207-08-9 Benzo(k)fluoranthene $10 \mathrm{U}$

50-32-8 Benzo(a)Pyrene $10 \mathrm{U}$

193-39-5 Indeno(1,2,3-cd)Pyrene $10 \mathrm{U}$

53-70-3 Oibenz(a, h)enthracene $10 \mathrm{U}$

191-24-2 Cenzolg,h,i)perylene $10 \mathrm{~J}$

(1)-Cannot be separated from diphenylasine 


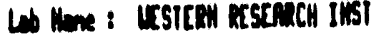
Case to : vill argnics malysis mith surel

(fone 2)

\section{Senivolatile Compounds}

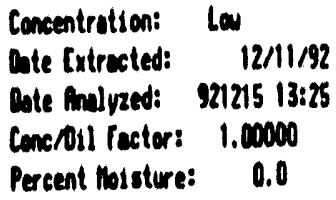

I Smole Murber I

I III

\author{
Seperatory rumel Extraction - Yes \\ Contimous Llouid-Liquid Extraction $X$ Yes
}

Yes $X X$

\section{C.A.S.}

Muber

100-95-2 Phenol

111-44-4 bis(2-Ch] oroethyl)ether

55-57-8 2-Ch] rophenol

511-73-1 1,3-Dichlerobenzene

106-46-7 1,4-0ichlorobenzene

55-50-1 1,2-0ichlorabenzene

95-19-7 2thethyl phenol

$100-60-1 \quad 2,2^{2}$-oxybis (1-Chlor opropene)

106-14-5 1thethylphenol

621-64-7 M-Hitroso-d1-n-prapylenine

67-72-1 Mexuchlor oethane

9.-95-3 Nitrobenzent

78-59-1 I cophor one

89-75-5 2-Hitrophenol

105-67-9 2,4-Dinethylphenol

$111-91-1$ bis(2-Chloroethoxymethone

120-63-2 2,4-0ichlorophenol

120-82-1 1,2,4-Irichlorobenzene

91-20-3 Maphthalene

106-47-8 4-Chloraniline

07-68-3 Vexochlopobutadiene

59-50-7 4-Chloro-3-3ethyl phenol

91-59-6 2tiethylnaphtholene

77-47-9 Hexachloracyclopentatiene

08-06-2 2,9,6-Irichlorophenol

\$5-\%5-4 2,9,5-Irichlor aphenol

91-50-7 2-Chloronaphthalene

88-74-4 2-listroandline

131-11-3 Dinethyl phthalate

208-\%-8 Renaphthylene

99-09-2 3-Nitrantline

83-32-9 Barmaphthene
$16 n$

C.A.S.

Muber

$40 / 1$

51-20-5 2,4-Ainitrophenol $25 \mathrm{U}$

100-02-7 4-litrophenol $25 \mathrm{U}$

132-64-9 Dibenzofuren $10 \mathrm{U}$

121-14-2 2,4-Dinitrotoluene $\quad 10 \mathrm{U}$

CO6-20-2 2,6-Dinitrotol uetie $\quad 10 \mathrm{~V}$

M-66-2 Diethylohthalote $10 \mathrm{U}$

7005-72-3 4-Chlorophenyl-ohenyletier $10 \mathrm{~V}$

65-73-7 rluorene $10 \mathrm{~V}$

100-01-6 4-Hitrosniline $25 \mathrm{~V}$

$534-52-1 \quad 4,6-0$ init tro-2-nethylphenol 25 U

6-30-6 H-Hitrosodiphenylemine (I) $10 \mathrm{U}$

101-55-3 4-Aronophenyl-phenylether $10 \mathrm{U}$

118-74-1 Mexachlorobenzene $10 \mathrm{U}$

87-86-5 Pentachlorophenol $25 \mathrm{U}$

85-01-8 Pheranthrene $10 \mathrm{~V}$

120-12-7 inthracene $10 \mathrm{~J}$

86-74-8 Carbozole $10 \mathrm{U}$

84-74-2 Di-n-Butylphthalate $\quad 1 \mathrm{~J}$

206-14-00 Iluoranthene $\quad 10 \mathrm{U}$

129-00-00 Pyrene $10 \mathrm{~V}$

85-68.? Dutylbenzylohthalate $10 \mathrm{U}$

91-94-1 3,3'-dichlorobenzidinz $\quad 10 \mathrm{U}$

56-55-3 Benzo(a) An thracene $10 \mathrm{U}$

117-81-7 bis(z-[thy]hexy]) phthalate $1 \mathrm{~J}$

218-01-9 Chryene $10 \mathrm{U}$

$117-84-00$ D D1-n-actulphthalate $10 \mathrm{U}$

205-99-2 Benzo(b) fluoranthene $10 \mathrm{U}$

207-08-9 Benzo(k)fluoranthene $10 \mathrm{~V}$

5n-32-8 Benzo(a)Purene $10 \%$

193-39-5 Indeno(1,2,3-cd)Pyrene $10 \mathrm{U}$

53-70-3 Dibenz(0,h)enthrecene $10 \mathrm{U}$

191-24-2 Benzolg,h,i)perylene $10 \mathrm{~V}$

(1)-Cannot be separated fron diphenylamsne 


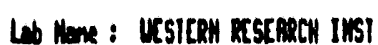

Cose No : Will
I smple Murber I

I Its

ansowics malysIs math sHett

(Page 2)

Senivolatile Conpounds

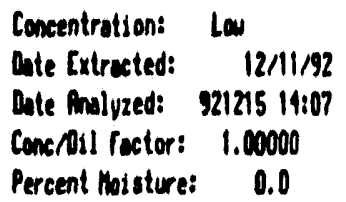

C.A.S.

Munber

151

108-95-2 Phenol

$111-14-4$ bis(2-Chloroethyl)ether

55-57-8 2-chlorophen:l]

541-73-1 1,3-0ichlordienzene

106-46-7 1,4-0ichlorobenzene

95-50-1 1,2-0ichlorabenzene

95-48-7 2thethylphenol

108-60-1 2,2'-oxybio(1-chloropropane)

106-14-5 it the thylohenol

$621-64-7$ H-Hitroso-di-n-propylenine

$67-72-1$ Mexechlor oethane

98-95-3 Mitrobenzene

78-59-1 Isophor one

88-75-5 2-Hitrophenol

105-67-9 2,4-0inethylphenol

$111-91-1$ b1s(2-Chloroe thaxy)nethene

120-83-2 2,4-Dichlor ophenol

120-82-1 1,2,4-irichlorobenzene

$91-20-3$ Maphtholene

106-97-8 4-Chloraniline

87-68-3 Hexachlorobutadiene

59-50-7 4-Chloro-3-nethyl phenol

91-57-6 2thethylnophthol ene

77-17-4 Nexchlorocyclopentadiene

80-06-2 2,1,6-1 richlor cophenol

55-55-4 2,4,5-Irichlorophenol

91-50-? 2-Chlor onsohthalene

89-74-4 2-Hitroaniline

131-11-3 Dinethylphthalote

209-\%8-8 Remaphthylene

99-09-2 3-Hitroniline

83-32-9 Acenaphthene gep Cleanup - Yes XX No

Separatory runnel Extraction - Yes

Continuous Liquid-Liquid Extraction $\perp$ Yes

C.A.S.

Nunber

15R

51-28-5 2,4-0initrophenol

100-02-7 4-Witraphenol

132-64-9 Dibenzoluran

121-14-2 2,4-Dinitrotoluene

606-20-2 2,6-0initrotoluene

64-66-2 Diethylphthalate

7005-72-3 4-Chiorophenyl-phenylether

86-73-7 Fluorene

100-01-6 4-Nitroonsline

534-52-1 4,6-Dinitrs-? nethylphenol

86-30-6 M-Hitrosodiphenylerine (1)

101-55-3 4-Bronopheny]-phenylether

118-74-1 Hexachlorobenzene

87-86-5 Pentachlor ophenol

85-01-8 Phenanthrene

120-12-7 inthracene

86-74-8 Carbazole

84-74-2 Di-n-Butylphthalate

206-44-00 rluorenthene

129-00-00 Purene

85-68-9 Butylbenzylphthalate

$91-94-1 \quad 3,31$-Dichlorobenzidine

56-55-3 Benzo(a) Anthracene

$117-81-7$ bis(2-Ethylhexyl) phthulate

210-01-9 Chrysene

117-84- $000 i-n-o c t y l$ ahthalate

205-99-2 Benzo(b)fluoranthene

207-00-9 Benzo(k)fluoranthene

50-32-8 Benzo(a) Pyrene

193-39-5 Indeno(1,2,3-cd)Purene

$53-70-3$ Dibenz $(0, h)$ onthr acene

191-24-2 Denzo(g,h,i)perylene

(1)-Camot be separated tron diphenylenine

$\begin{array}{ll}25 & U \\ 25 & U \\ 10 & U \\ 10 & U \\ 10 & U \\ 10 & U \\ 10 & U \\ 10 & U \\ 25 & U \\ 25 & U \\ 10 & U \\ 10 & U \\ 10 & U \\ 25 & U \\ 10 & U \\ 10 & U \\ 10 & U \\ 1 & J \\ 10 & U \\ 10 & U \\ 10 & U \\ 10 & U \\ 10 & U \\ 1 & J \\ 10 & U \\ 10 & U \\ 10 & U \\ 10 & U \\ 10 & U \\ 10 & U \\ 10 & U \\ 10 & U\end{array}$

in 11 
Lat Hine: : Uestern Rescrech IMST

Cose tho:

cosmics malysis Dain SHeEI

1 semple Munber 1

I IUII

(Poge 2)

Sentuolotile Compounds

\begin{abstract}
Concentration: Law
Gate Extracted: 12/11/92

Date Rnalyzed: 921215 14:50

Cone/Dil lactor: 1.00000

Percent Moisture: $\quad 0.0$
\end{abstract}

GPC Cleanup - Yes Ho

Separatory rumel Extraction _ Yes

Continuous Liquid-Liquid Extraction $\perp$ Yes
C.A.S.

Humber

108-95-2 Phenol

$111-44-4$ bis(2-Chloroethyl)ether

95-57-8 2-chlorophenol

591-73-1 1,3-0ichlorobenzene

106-96-7 1,4-0ichlorobenzene

\$5-50-1 1,2-01chlorabenzene

95-48-7 2-tiethylphenol

108-60-1 2,2'-oxpibis(1-Chlop opropane)

$206-14-5$ thethylphenol

621-64-7 H-Hitroso-di-n-propylenine

67-72.1 Hexachlor oethane

98-95-3 Hatrobenzene

$78-59-1$ Isophorone

88-75-5 2-Hitrophenol

105-67-9 2,1-Dine thylphenol

$111-91-1$ bis(2-Chlor oethoxy)methane

120-83-2 2,4-0ichlor ophenol

120-82-1 1,2,4-1richlorobenzene

91-20-3 Maphthalene

106-47-8 4-Chloraniline

87-68-3 Mexactisorobutadiene

59-50-7 4-Chioro-3-nethyl phenol

91-57.6 2thethylnephthalene

77-47-4 Mexachlor ocyclopentadiene

68-06-2 2,4,6-1 richlorophenol

95-55-1 2,1,5-Irichlor optienol

91-58-7 2-Chloronephthalene

06-74-4 2-Mitraansline

131-11-3 Dinethylohthalate

200-96-8 Reenephthylene

99-09-2 3-Hitrooniline

83-32-9 Reensphthere
1611

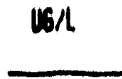

$\begin{array}{ll}10 & U \\ 10 & U \\ 10 & U \\ 10 & U \\ 10 & U \\ 10 & U \\ 10 & U \\ 10 & U \\ 10 & U \\ 10 & U \\ 10 & U \\ 10 & U \\ 10 & U \\ 10 & U \\ 10 & U \\ 10 & U \\ 10 & U \\ 10 & U \\ 10 & U \\ 10 & U \\ 10 & U \\ 10 & U \\ 10 & U \\ 10 & U \\ 10 & U \\ 25 & U \\ 10 & U \\ 25 & U \\ 10 & U \\ 10 & U \\ 25 & U \\ 10 & U \\ & \end{array}$

C.A.S.

Nunber

51-28-5 2,4-Dinitrophenol $25 \mathrm{U}$

100-02-7 4-Hitrophenol $25 \mathrm{U}$

132-64-9 Dibenzofuran $10 \mathrm{U}$

121-14-2 2,4-0initrotoluene $10 \mathrm{U}$

606-20-2 2,6-Dinitrotoluent $\quad 10 \mathrm{U}$

84-66-2 Diethylphthalote $10 \mathrm{U}$

7005-72-3 4-Chlorophenyl-ohenylether $10 \mathrm{~V}$

86-73-7 Fluorene $10 \mathrm{U}$

100-01-6 4-Hitroaniline $25 \mathrm{U}$

531-52-1 1,6-0inatro-2-nethulphenol $25 \mathrm{U}$

B6-30-6 $\mathrm{H}$ - Hitrosodsphenylemine (1) $10 \mathrm{~V}$

101-55-3 4-Ermophenyl-phenylether $10 \mathrm{~V}$

118-74-1 Hexachiorobenzene $10 \mathrm{~V}$

87-86-5 Pentachlorophenol $25 \mathrm{U}$

85-01-8 Phenonthrene $10 \mathrm{U}$

120-12-9 Anthracene $10 \mathrm{U}$

86-74-8 Carbazole $10 \mathrm{U}$

84-74-2 Di-n-butylphthalate $1 \mathrm{~J}$

206-44-00 Fluoranthene $10 \mathrm{U}$

129-00-00 Purene $10 \mathrm{~V}$

85-68-7 Butylbenzylohthalate $\quad 10 \mathrm{U}$

91-94-1 3,3'-Dichlorobenzidine $\quad 10 \mathrm{~V}$

56-55-3 Benzo(a)Anthracene $10 \mathrm{U}$

$117-81-?$ bis(2-[thylhexyl) phthalate $\quad 0.7 \mathrm{~J}$

218-01-9 Chrysene $10 \mathrm{U}$

$117-84-00$ Di-n-octylphthalate $\quad 10 \mathrm{U}$

205-99-2 Benzo(b)fluoranthene $10 \mathrm{U}$

207-09-9 Benzo(k)fluoranthene $10 \mathrm{U}$

50-32-8 Benzo(a)Pyrene $10 \mathrm{U}$

193-39-5 Indeno(1,2,3-co)Purene $10 \mathrm{U}$

53-70-3 Dibenz(a, h)enthracene $10 \mathrm{~V}$

191-24-2 Benzolg, h, 1) perylene io U

(1)-Cannot be separated from diphenylamine 


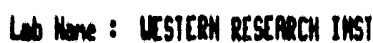

Cone tho : Mil

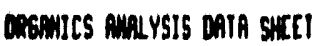

1 smple Muber 1

I IUI2

(Paxe 2)

Senivolatile Compounds

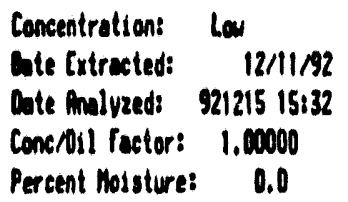

spe Cleanup - Yes Wo No Separatory funnel Extraction - Yes Continuous Liquid-Liquid Extraction $X_{Y e s}$

C.A.S.

C.A.S.

1061

Number

$16 / \mathrm{L}$

\section{8-95-2 Phenol}

$111-44-4$ bis (2-CAloroethyl)ether

$10 \mathrm{~V}$

$10 \mathrm{~V}$

$10 \mathrm{U}$

95-57-8 2-Chlorophenol

511-73-1 1,3-Dichlorobenzene

106-46-9 1.4-Dichlorobenzene

95-50-1 1,2-0ichlorobenzene

95-48-7 2tiethylphenol

108-60-1 2,2'-oxybis(1-Chloropropene)

106-14-5 4thethylphenol

621-64-7 H-Witroso-di-n-propylanine

67-72-1 Hexachloroethane

99-95-3 Notrobenzene

78-59-1 Ioophorone

88-75-5 2-Nitrophenol

105-67-9 2,4-Dinethylphenol

$111-91-1$ - bis (2-Chloroethoxy) methene

120-83-2 2,4-Diehloropheno!

120-82-1 1,2,4-Irichlor obenzene

91-20-3 Maphthalene

106-17-8 4-Chloraniline

87-68-3 Hexachlorobutadiene

59-50-7 4-Chloro-3-methylahenol

91-57.6 2thethyl nophtholene

77-49-4 Mexactlorocyclopentediene

88-06-2 2,1,6-Irichlor ophenol

55-95-4 2,1,5-Irichlorophenol

91-56-7 2-Chloronaphthalene

89-74-4 2-Hitroeniline

131-11-3 Binethylphthalate

208-96-8 Acenaphthylene

95-09-2 3-tibitroaniline

33-32-9 Aceraphthene

\begin{tabular}{|c|c|c|}
\hline $51-28-5$ & 2,4-Dinitrophenol & 25 \\
\hline $100-02-7$ & 4-Witrophenol & 25 \\
\hline $132-64-9$ & Dibenzofuran & 10 \\
\hline$|2|-14-2$ & 2,4-Oinitrotoluene & 10 \\
\hline $606-20-2$ & 2,6-Dinit trotoluene & 10 \\
\hline $94-66-2$ & Diethylphthalate & 10 \\
\hline $7005-72-3$ & 4-Chlor ophenyl - phenylether & 10 \\
\hline $86-73-7$ & fluorene & 10 \\
\hline $100-01-6$ & 4-Hitroansline & 25 \\
\hline $534-52-1$ & 1,6-Dins tro-2 nethylphenol & 25 \\
\hline $86-30-6$ & N-Kitrosodiphenylmine (1) & 10 \\
\hline $101-55-3$ & 4-Bronophenyl-phenylether & 10 \\
\hline $118-74-1$ & Mexachlorobenzene & 10 \\
\hline $87-86-5$ & Pentachlor ophenol & 25 \\
\hline $05-01-8$ & Phenanthrene & 10 \\
\hline $120-12-7$ & Anthracene & 10 \\
\hline $86-74-8$ & Carbazole & 10 \\
\hline $84-74-2$ & Di-n-Butulphthalate & 1 \\
\hline $206-14-00$ & fluoronthene & 10 \\
\hline $129-00-00$ & Purene & 10 \\
\hline $85-68-7$ & Butylbenzylphthalate & 10 \\
\hline $91-94-1$ & 3,3'-Dichlorobenzidine & 10 \\
\hline $56-55-3$ & Benzola) Anthracene & 10 \\
\hline $117-81-7$ & bis(2-[thylhexyl) phthalate & 1 \\
\hline $218-01-9$ & Chrysene & 10 \\
\hline $117-84-00$ & Di-n-octylphthalate & 10 \\
\hline $205-99-2$ & Denzo(b) fluor anthene & 10 \\
\hline $207-00-9$ & Cenzo(h) fluoranthene & 10 \\
\hline $50-32-8$ & Benzo(a)Pyrene & 10 \\
\hline $193-39-5$ & Indeno $(1,2,3$-cd)Pyrene & 10 \\
\hline $53-70-3$ & Dibenz (a, h) anthracene & 10 \\
\hline $191-24 \cdot 2$ & Benzo $(g, h, i)$ perylene & 10 \\
\hline
\end{tabular}

Porn I 
Lo ture : Irsten ersserach INST Cace No: ill

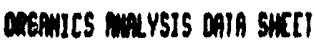

(Poge 2)

\section{Seniculatile Compounds}

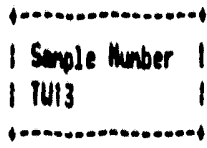

[....................

\begin{tabular}{|c|c|}
\hline $\begin{array}{l}\text { Concentration: } \\
\text { Cate Ixtracted: } \\
\text { Oale mulyzed: } \\
\text { Conc/Oil factor: } \\
\text { Percent hosotures }\end{array}$ & $\begin{array}{l}\text { lod } \\
12 / 11 / 92 \\
92121516: 15 \\
1.00000 \\
0.0\end{array}$ \\
\hline
\end{tabular}

Cec Clemun Yes Ho Separalory funnel Extraction - Yes Continuous Liquid-Liquid Extraction \& Yes

\section{C.R.S.}

Number

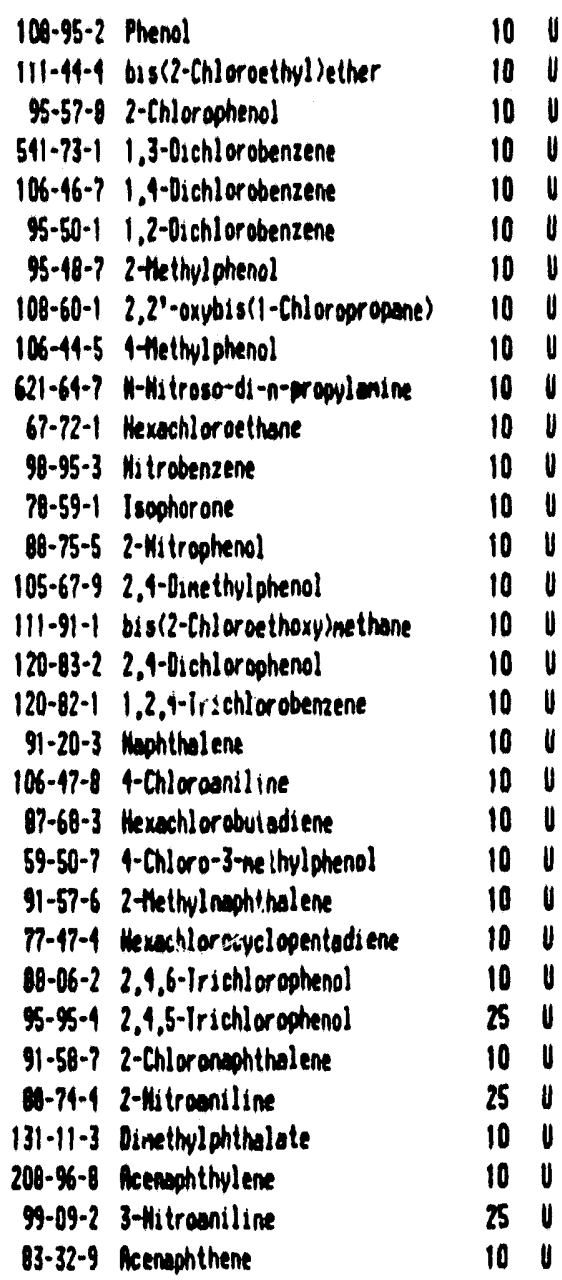

\section{C.A.S.}

Munber

$16 / 1$

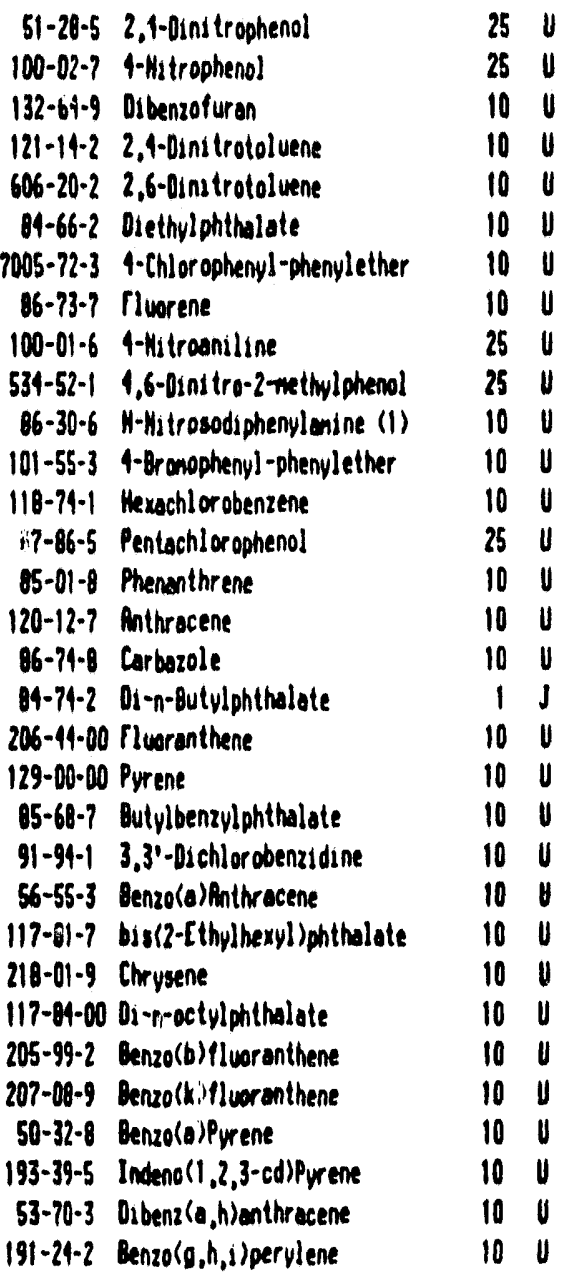

(1)-Connot be ceparated from diphenylanine 


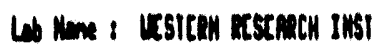
Cone No : vil

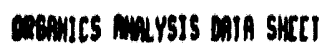
(Rae 2)

Senivolotile conpounds
I Suple Muber I
OPC Clamup - Yes W Ho

Senaratory funcl Extraction - Yes

Continuous Liquid-Liquid Extraction $X$ Yes

ate malyzed: 921215 16157

conc/01] fector: 1,00000

Percent folstures 0.0

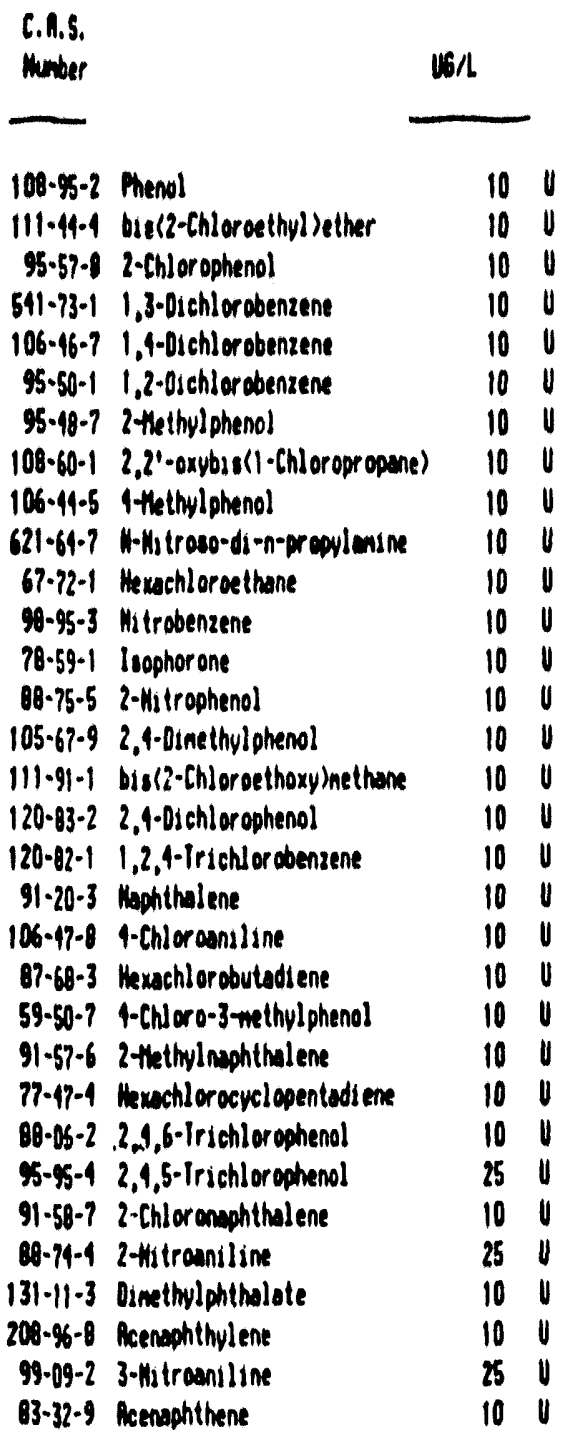

C.R.S.

Munber

$16 /$

$51-28-52,4-0$ initrophenol

100-02-7 4-1litraphenol

132.64.9 Dibenzofuran

$121-14-2 \quad 2,4-$ Dinstrololuene

606-20-2 2,6-0ins trotoluene

04-66-2 Diethylphilhalate

2005-72-3 1-Chlor ophenyl-ohenyle ther

06-73.7 Tluorene

100-01-6 4-Nitransline

$534-52-1 \quad$ 1,6-0ins tro-2-nethylphenol

86-30-6 N-Hitrosodiphenylemine (1)

101-55-3 1-8romophenyl-ohenyle ther

118-74.1 Hexachlor obenzene

87-86-5 Pentachlorophenol

55-01-8 Phenenthrene

120-12-7 inthracene

86-74-8 Carbazole

84-74-2 Oi-n-Dutylphtholate

206-44-00 rlueranthene

129-00-00 Purene

85-68-7 Butylbenzylphthalale

91-94-1 3,3'-Dichlordbenzidine

56-55-3 Benzo(a) Anthracene

$117-81-7$ bis(2-[thylhexyl) anthalate

218-01-9 Chrysene

117-84-00 01-n-octulphthalate

205-99-2 Eenzo(b) fluorenthene

207-08-9 Benzo(h) flluoranthene

50-32.8 Benzo(a)Purene

193-39-5 Indeno(1,2,3-cd)Purene

$53-70-3$ Oibenz $(a, h)$ anthracene

191-24-2 Benzo< $g, h, i)$ perylene

(1)-Cannot be separated from diphenylanine

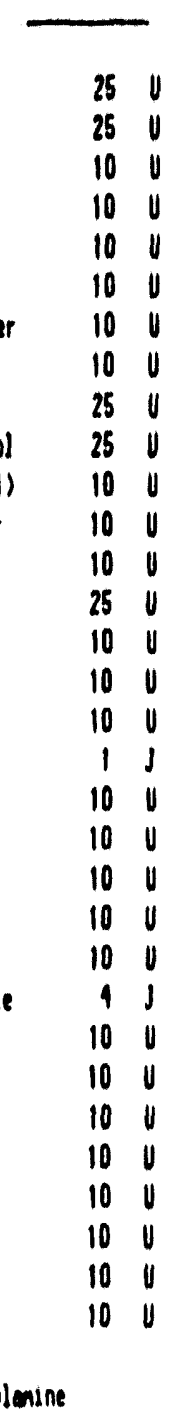

forn 1 


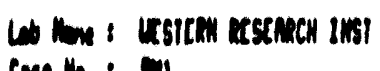

can in : nil

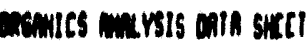

(Rom ?)

Senivolotsle tompounds

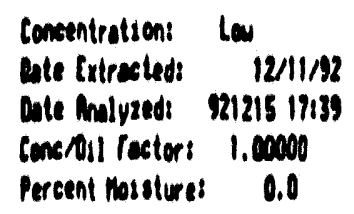

exc clemis - Yes wo

Anpotory fumel cutraction - Yes

Conlinuous Liqud-Liquid extraction $Y$ Yes
C.A.S.

Munber

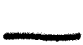

109-95-2 Phenol

111-44-4 bis(2-Chloroethy!)ether

\$5-57-8 2-chlorophenal

$511-73-1$ 1,3-0ichlorobenzens

106-16-7 1,4-01chlopobenzene

\$5.50-1 1,2-01chlorobenzene

95-18-9 2thethylohenol

100-60-1 2,2'-orybis(1-chloropreanes)

$106-14-5$ thethylphenol

(2)-64-7 N-Nitroso-ds-n-prepularine

67.72 .1 Hexachlor oethane

98-95.3 Malrobenzene

78-59-1 loophorone

88.75.5 2-Hitrounenol

105.67-9 2,4-Dinethylohenol

$111.91-1$ bio(2-Chloroethoxy) methane

120-83-2 2,4-Dichlorophenol

$120 \cdot 82-1 \quad 1,2,4-1$ richlor obenzene

$91-20-3$ Nephthalene

106-47-8 4-Chlorantiline

$07-60.3$ Hexchlordoutadiene

59-50-7 4-Chloro-3-3nethylohenol

91.57-6 2thethy Inehthalene

n-17-4 Wuxchlor covelopentadiene

06-06-2 2,4,6-Irichler cophenal

\$5. 5 -4 2,4,5-Irichleropheno!

91-50-7 2-chleranohthalene

4.74-4 2-Nitranstine

$|3|-\mid 1-3$ Oinethylonthalate

200-\%+8 nemaphthylene

99.09-2 3-Hitranilime

03-32.9 Acenophthene
161

G.A.S.

Musuer

$16 / 1$
1 sonple Muber 1

11016
1 
Wh thene : Usten kexench Inst Con No : All

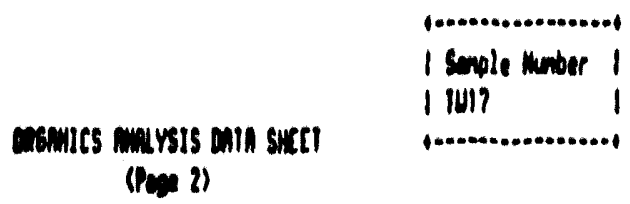

Sentwolutile Compands
Cit Clemen - Yes in in Enerotory funmel tritroction _ Yes continuous Liqusd-Liquid trutrestion $x$ Yes
Comentralions la
cte critresteds 12/11/
Oate mulysed: 2212151120
conenoil festers 1.0000
Percent mistotures

C.A.S.

Munber

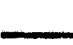

100-95-2 Phenol

$111-14-4$ biol2-chlorathyl)ether

55.57-1 $2 \cdot$ Chlorophenol

$511 \cdot 73 \cdot 1$ 1,3-Oichlorobenzene

$106-16-7$ 1,1-O1chlorobenzene

95-50-1 1,2-01chlorobenzene

95.48-7 2thethylphenol

108-60-1 2,2'-oxybsell-chloropropene)

106-11-6 the thylohenol

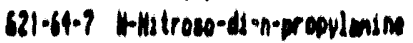

$67.72-1$ Mexchloroethone

9. 55 -3 Hitrobenzene

$78-59-1$ Isophorone

80-75-5 2-Nitrophenol

105-67-9 2,4-Dine thylphenol

$111 \cdot 91 \cdot 1$ bis(2-Chloroethoxy)hethene

[20-83-2 2,4-0ichlorophenol

$120 \cdot 82-1 \quad 1,2,4 \cdot$ Irichlorobenzene

91-20-3 Nophthalene

106-17-8 4-Chlorantline

87-68-3 Hewachlorobutadiene

59-50-7 4-Chloro-3-3nethylohenol

$91-57.62$ inethylmaphthalene

$77-47-4$ Vexchlorocyclopentadiene

88-06-2 2, 1,6-Irichlorophenol

\$5-55-4 2,9,5-Irichlorophenol

91-58-9 2-Chloroneotholene

20-74-4 2-Hitrandine

$|3|-11-3$ Dimelhylohthalate

200-\%8-8 Remephthylene

99.09-2 3-Hitraniline

13-32-9 Renaphthene
1814

$10 \mathrm{~V}$

$10 \mathrm{~V}$

$10 \mathrm{~V}$

$10 \mathrm{~V}$

$10 \mathrm{~V}$

$10 \mathrm{~V}$

$10 \mathrm{U}$

$10 \mathrm{~V}$

$10 \mathrm{U}$

$10 \mathrm{~V}$

C.A.S.

Number

$51-20-5 \quad 2,4-0$ ins trophenol

100-02-7 4-llitrophenol

132.64-9 Dibenzofuron

$121 \cdot 14-2 \quad 2,4 \cdot 0$ instrotoluene

606-20-2 2,6-Dinitrotoluene

04-66-2 Oiethyiphthalote

$7005-72-3$ 4-Chlorophenyl-ohenylether

6-73-7 fluorene

100-01-6 4-Hitraensiline

534-52-1 4,6-0initro-2nethyldinenol

\%.30.6 N-Nitrosodidienularine (1)

101-55-3 4-Eromophenyl-phenylether

118-74.1 Mexachlorobenzene

17-06-5 Pentachlorophenol

$5.01 \cdot 8$ Phementhrene

120-12-7 Anthrecene

46.74-8 Corbazole

$04-74-2$ D2-n-Butylanthalate

206-44-00 rluoranthene

129-00-00 Pyrene

65-68-7 Dutylbenzylohtholate

\$1-94-1 3,3'-Dichlorobenzidine

s6-55-3 Cenzo(a) Pinthracene

117-91-? bis(2-[thylhexyl)phthalate

210-01-9 chrveme

$117-84-0002-n-$ octylohtholate

205-99-2 cenzo(b) fluoranthene

207-00-9 cenzo(k)fluoranthene

50-32-8 cenzo(a)Pivene

193-39-5 Indeno(1,2,3-cd)Pyrene

53-70-3 Oibuna (o, h) menthracene

$191-24-2$ Benzo(g,h, 1$)$ pervlene

$10 \mathrm{~V}$

$10 \mathrm{~V}$

$10 \mathrm{~V}$

$10 \mathrm{~V}$

$10 \mathrm{~V}$

$10 \mathrm{~V}$

$25 \mathrm{~V}$

$25 V$

$10 \mathrm{~V}$

$10 \mathrm{~V}$

$10 \mathrm{~V}$

$25 V$

$10 \mathrm{~V}$

$10 V$

$10 \mathrm{~V}$

$1 \mathrm{~J}$

$10 V$

$10 \mathrm{~V}$

$10 \mathrm{~V}$

(1)- Comot be suparated tran diphenyleuine 


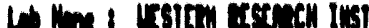
can In 1 inl

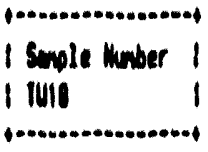

menuses maysis gith sortl (Rave 2)

Enivolatile tompounds

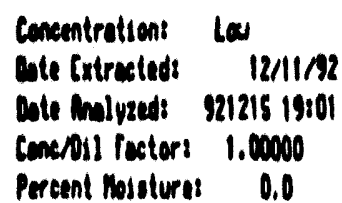

C.A.S.

Nunber

1516

100-45.2 Phenel

$111-14-1$ bis(2-Chloroethyl)ether

X5.57-8 2-CNlopaphenol "

511-73-1 1,3-01chlorobenzene

$106-16-7 \quad 1,4-01$ chlopobenzene

55-50-1 1,200ichlorobenzene

\%.48-? 2 thethylohenol

108-60-1 2, 2'-oxybio(1-Chlorepropene)

106-14-5 thethylphenol

(21-64-7 H-Wtroso-ds-n-propylouine

69.72.1 Mexechloroethene

98-95-3 Hitrobenzene

78-59-1 I cophor one

Bo-75-5 2-Kitrophenol

105-67-9 2,4-Dinethylohenol

$111-91-1$ bus(2-Chloroethoxy)methane

120-83-2 2,1-Dichlorophenol

120-82-1 1,2,4-1 richlordobenzent

$91 \cdot 20 \cdot 3$ Nempthaleme

106-47.8 4-Chloransline

07-60-3 Hewachlordoutadieme

59-50-7 4-Chloro-3-methyl phenol

$91-57.62$ thethylnaphthalene

77-17-4 Menchleroovelosentadiene

00-06-2 2,1,6-Irlatiloraghenel

\$5-\$5-4 2,4,5-irichlerophanol

91-58-9 2-Chleronehthalene

9-74-9 2-Nistraniline

$|3|-\mid 1-3$ oine thylphtholote

200-9s-8 cencophthylene

99-09-2 3-litranilian

33-32-9 Recruphthene
$10 \mathrm{~V}$

$10 \mathrm{~V}$

100

$10 \mathrm{~V}$

$10 \mathrm{~V}$

$10 \mathrm{~V}$

$10 \mathrm{~V}$

$10 \mathrm{~V}$

$10 \mathrm{U}$

$10 \mathrm{~V}$

$10 \mathrm{~V}$

$10 \mathrm{U}$

$10 \mathrm{~V}$

$10 \mathrm{~V}$

$10 \mathrm{~V}$

$10 \mathrm{~V}$

$10 \mathrm{~V}$

$10 \mathrm{~V}$

$10 \mathrm{~V}$

$10 \mathrm{~V}$

$10 \mathrm{~V}$

$10 \mathrm{~V}$

$10 \mathrm{~V}$

$10 \mathrm{~V}$

$10 \mathrm{~V}$

$25 \mathrm{~V}$

$10 \mathrm{~V}$

25

$10 \mathrm{~V}$

$10 \mathrm{~V}$

$25 \mathrm{~V}$

100

\author{
ect Cleanue - Yes Wo \\ Sapurolory funcl cutraction - Yes \\ Contimous Liquidrliquid Extraction $X$ Yes
}

C.A.S.

Huaber

$16 / 1$

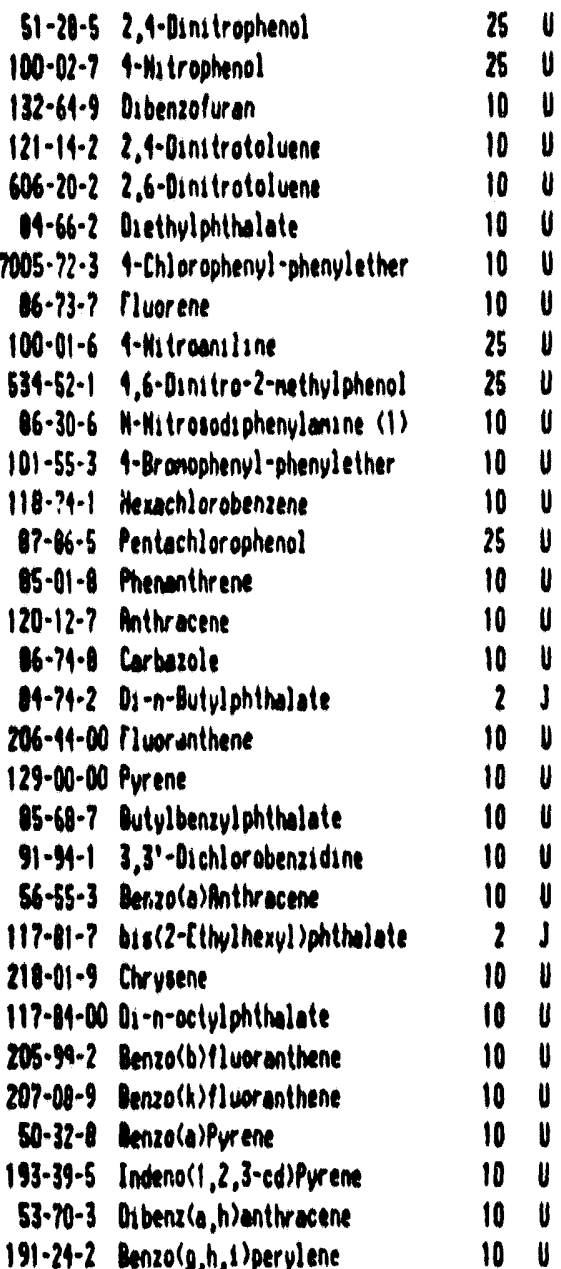

(1)-Cumot be separated tron diphenylewine 
La Man: Irstern Restemen Inst

Cane No $:$ Mil macevics maiysis caita suret

(Rin 2)

Senjuolatile Compounds

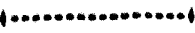

I smple Muber 1

1 1430

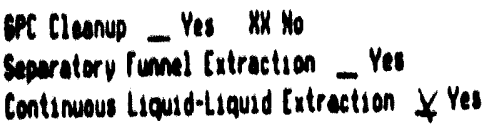

C.A.S.

Number

$46 / 1$

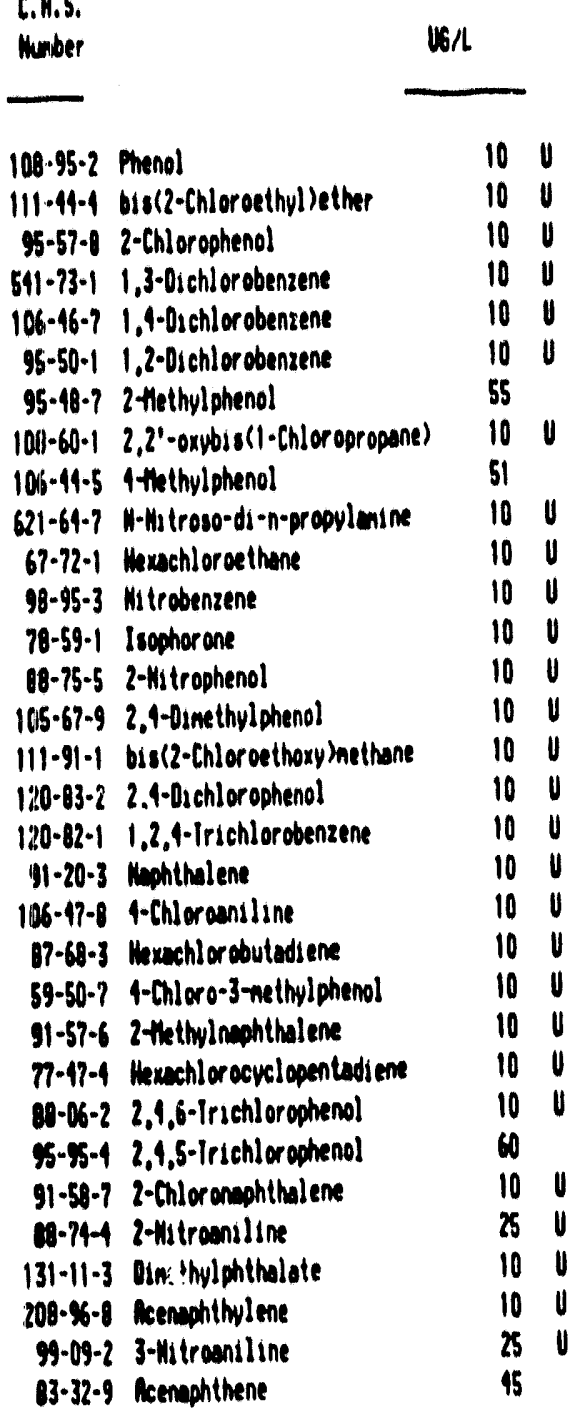

$51-20-52,4-0$ initrophenol $25 \mathrm{U}$

100-02-7 4-Hatrophenol $25 \mathrm{U}$

132-64.9 Oibenzofuran $10 \mathrm{U}$

$121 \cdot 14-2 \quad 2,4-$ Dinsitrotoluene $\quad 10 \mathrm{U}$

$606-20-2 \quad 2,6-0$ initrotoluene $10 \mathrm{U}$

94-66-2 Diethylphthalale $10 \mathrm{~V}$

7005-72-3 4-Chlorophenyl-phenylether $10 \mathrm{U}$

86.73 .9 Pluorene $10 \mathrm{U}$

100-01-6 4-Hitroaniline $25 \mathrm{U}$

534-52-1 1,6-0initro-2-nethylphenal $25 \mathrm{~V}$

06-30-6 N-Hitrosodiphenylerine (I) $10 \mathrm{U}$

101-55-3 4-Bu anophenyl-pirenylether $10 \mathrm{U}$

118-24.1 Kexachlorobenzene $10 \mathrm{~V}$

87.86.5 Pentachlorophenol $25 \mathrm{~V}$

85-01-8 Phenenthrene $10 \mathrm{U}$

120.12.7 Anthracene 10

06-74-8 Carbazole $10 \mathrm{U}$

84-74-2 $01-n-$ Eutylohthalate 11

206-44-00 Fluorenthene $10 \mathrm{~V}$

129-00-00 Pyrene $10 \mathrm{U}$

85-68-7 Butylbenzylphthalate $10 \mathrm{U}$

$91-94-1$ 3,3'-Dichlorobenzidzne $\quad 10 \mathrm{~V}$

56-55-3 Benzo(a) Anthrocene $10 \mathrm{U}$

117-81-7 bis(2-[thylhexyl) phithiole $1 \mathrm{~J}$

218-01-9 Chrysene $10 \mathrm{U}$

$117-84-00$ 0 $01-n-0 c t y i$ phthalate $\quad 10 \mathrm{U}$

205-49-2 Benzo(b)fluoronthene $10 \mathrm{~V}$

207-09-9 Benzo(k)fluoronthene $10 \mathrm{U}$

50-32-8 Denzo(a)Pyrene $10 \mathrm{U}$

193-39-5 lindeno(1,2,3-cd)Purene $10 \mathrm{U}$

53-70-3 Dibenz(o, h)enthracene $10 \mathrm{~V}$

191-24-2 Benzolg,h, $)$ perylene $10 \mathrm{~V}$

(1)-Cannot be separated from diphenyleasne 
Lob Nowe: UsSIERH Restrixch IIST Case Ho:

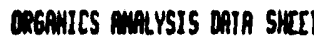

(Page 2)

Senivolatile Compounds
ePC Cleanus - Yes XX No

Separatory Fumel Extraction _ Yes Continuous Liquid-Liquid Extraction $X$ Yes
Cate Pinalyzed: 921215 20:24

conc/Dil factor: 1.00000

Pereent Moisture: $\quad 0.0$ i Somple Muber 1

| I I 31

C.A.S.

101

C.A.S,

Nunber

$46 / 2$

\begin{tabular}{|c|c|c|}
\hline $100-95-2$ & Phenol & 10 \\
\hline $111-4-4$ & bis(2-Chloroethyl)ether & 10 \\
\hline $95-57-8$ & 2-chlorophenol & 10 \\
\hline $541-73-1$ & 1,3-Dichlorobenzene & 10 \\
\hline $6-46-7$ & 1.1-Dichlorobenzene & 10 \\
\hline $95-50-1$ & 1,2-0ichlorobenzene & 10 \\
\hline $95-48-7$ & 2 thethyl phenol & 10 \\
\hline $100-60-1$ & $2,2^{\prime}$-oxybis(1-Chloropropane) & 10 \\
\hline $106-44-5$ & 1-thethylphenol & 10 \\
\hline $621-64-7$ & Wistroso-di-n-propularine & 10 \\
\hline $67-72-1$ & Mexachloroethane & 10 \\
\hline $98-95-3$ & Nitrobenzene & 10 \\
\hline $78-59-1$ & Isophor one & 10 \\
\hline $88-75-5$ & 2-Hitrophenol & 10 \\
\hline $105-67-9$ & 2,4-Dinethyl phenol & 10 \\
\hline $111-91-1$ & bis(2-Chlor oethoxy) methane & 10 \\
\hline $120-83-2$ & 8,4-D,chlor ophenol & 10 \\
\hline $120-82-1$ & 1,2,4-irichlorobenzene & 10 \\
\hline $91-20-3$ & Maphthalene & 10 \\
\hline $106-47-8$ & 4-Chloroaniline & 10 \\
\hline $87-68-3$ & Hexachlor cbutadiene & 10 \\
\hline $59-50-7$ & 4-Chloro-3-methylphenol & 10 \\
\hline $91-57-6$ & 2 Hethylnaphthalene & 10 \\
\hline $77-17-1$ & Hexachlor ocyclopentodiene & 10 \\
\hline $88-06-2$ & 2,4,6-Irichlorophenol & 10 \\
\hline $95-95-4$ & 2,4,5-Irichlorophenol & 25 \\
\hline $91-58-7$ & 2-Chloronephthalene & 10 \\
\hline $88-74-4$ & 2-Hitrosniline & 25 \\
\hline$|3|-\mid 1-3$ & Dinethylphthalale & 10 \\
\hline $208-\%-8$ & Reensphthylene & 10 \\
\hline $99-09-2$ & 3-Hitraniline & 25 \\
\hline$-32-9$ & Acenaph thene & 10 \\
\hline
\end{tabular}

51-28-5 2,4-0initrophenol

100-02-7 4-Hitrophenol

132-64-9 Dibenzoluran

121-14-2 2,4-Dinatrotoluene

606-20-2 2,6-Dinitrotoluene

84-65-2 Oiethylphthalate

7005-7i-3 4-Chlor ophenyl-phenyle ther

86-73-7 Fluorene

100-01-6 4-Hitroondiline

534-52-1 1,6-Dinitro-2 methylphenol

86. 3-6 M-Hitrosodiphenylemine (1)

101-55-3 4-Bromophenyl-phenylether

118-74-1 Hexachlorobenzene

87-86-5 Pentachlorophenol

85-01-8 Phenanthrene

120-12-7 Anthracene

86-74-8 Carbozole

84-74-2 Bi-n-Butylphthalate

206-14-00 Fluor anthene

129-00-00 Pyrene

85-68-7 Butylbenzylphthalate

91-94-1 3,3'-Dichlorabenzidine

56-55-3 Benzo(a) inthracene

$117-81-?$ bis (2-[thylhexyl) phthalate

218-01-9 Cirysene

$117-84-0001$-n-octylphthalate

205-99-2 Benzo(b)fluoranthene

207-00-9 Renzo(k)fluoranthene

50-32-8 Benzo(a)Pyrene

193-39-5 Indeno(1,2,3-cd)Purene

53-70-3 Oibenz (a, h) enthrecene

191-24-2 Benzolg, h, i) perylene

250

$25 \mathrm{~V}$

$10 \mathrm{~V}$

$10 \mathrm{U}$

$10 \mathrm{~V}$

$10 \mathrm{~V}$

$10 \mathrm{~V}$

$10 \mathrm{~V}$

$25 \mathrm{~V}$

$25 \mathrm{~V}$

$10 \mathrm{U}$

$10 \mathrm{~V}$

$10 \mathrm{~V}$

$25 \mathrm{~V}$

$10 \mathrm{U}$

$10 \mathrm{~V}$

$10 \mathrm{~V}$

$1 \mathrm{~J}$

$10 \mathrm{~V}$

$10 \mathrm{U}$

$10 \mathrm{U}$

$10 \mathrm{~V}$

$10 \mathrm{~V}$

11

(1)-Cunnot be separated fron diphenylenine 


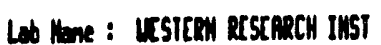
Cose Ho: ail

, arsouics miniysis dait sirtl

(Pege 2)
I Smple number 1

I 1432

\section{Seniculatile Compounds}

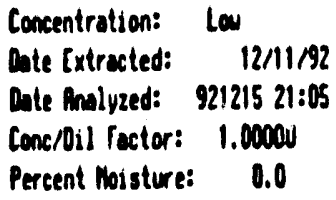

spC Cleanup - Yes XX no Separatory funnel extraction - Yes Contimous Liqued-Luquid Extraction $X$ Yes
C.A.S.

Number
$10 / 1$

108-95-2 Phenol

$111-44-4$ bis (2-Chloroethyl)ether

95-59-8 2-Chlor ophenol

511-73-1 1,3-02chlorobenzene

106-46-7 1,4-0ichlorobenzene

95-50-1 1,2-0ichlorobenzene

95-48-7 2-Hethylphenol

100-60-1 2,2'-oxybis(1-Chlor opropone)

106-41-5 4thethylphenol

621-64-? H-Hitroso-d1-n-propylerine

$67-72-1$ Hexachloroethane

98-95-3 Nitrobenzene

78-59-1 Isophorone

88-75-5 2-Hitrophenol

105-67-9 2,4-Dine thylphenol

$111-91-1$ bis(2-Chloroethoxymethene

120-83-2 2,4-0ichlor ophenol

120-82-1 1,2,4-Irichlorabenzene

91-20-3 Mophthalene

106-47-8 4-Chlor caniline

87-68-3 Mexchlorobutadiene

59-50-7 4-Chioro-3-methylphenol

91-59-6 2thethylnephthalene

77-17-4 Mexactlorocyclepentadiene

00-0s-2 2,1,6-Irichlor ophenol

S5-95-4 2,4,5-Irichlerophenol

91-50-7 2-Chloronohthalene

03-74-4 2-Hitromiline

131-11-3 Orethylphenalete

205-\%-8 icenonthylene

99-09-2 3-Hitreniline

03-32-9 Rcenaphthene

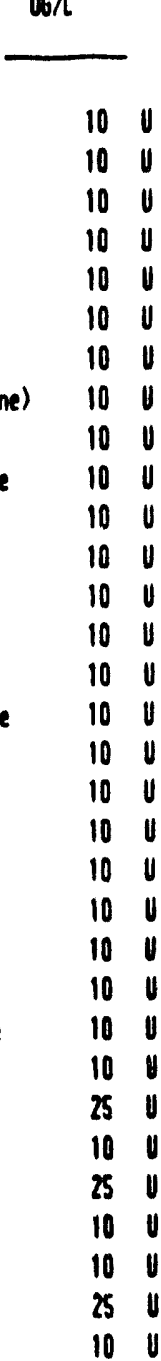

C.R.S.

Nunber

$16 / 1$

51-28-5 2,4-Dinitrophenol

100-02-7 4-Hatrophenol

132-64-9 Dibenzoluran

121-14-2 2,4-Dinitrololuene

$606-20-2 \quad 2,6-0$ initrotoluene

04-66-2 Diethylphthalate

2005-72-3 1-Chlor ophenyl-phenylether

86-73-7 fluorene

100-01-6 4-H1 troaniline

534-52-1 4,6-Dinitro-2-nethyl phenol

06-30-6 $N$ - $H_{1}$ trosodiphenylamine (1)

101-55-3 4-Branophenyl-phenylether

118-74-1 Hexachlorobenzene

87-86-5 Pentachlorophenol

05-01-8 Phenenthrene

120-12-7 Anthracene

06-74-8 Corbazole

84-74-2 Oi-n-8utylohthalate

206-44-00 rluorenthene

129-00-00 Purene

65-68-7 Butylbenzylohthalate

91-94-1 3,3'-Dichlorobenzidine

56-55-3 Benzo(a)inthracene

$117-81-7$ bas (2-[thylhexyl) ph thalate

210-01-9 Chrysene

$117-84-00$ D 1 -n-ectylphthalate

205-99-2 Benzo(b) fluoranthene

207-00-9 Cenzo(k) fluoranthene

SD-32-8 Cenzo(a) Purene

193-39-5 Indeno(1,2,3-cd)Pyrene

53-70-3 Dabenz(a,h)enthracene

191-24-2 Renzo(g,h,i)perylene

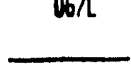

(1)-Cannot be separated fron diphenylanine

Iorn 1

$7 / 85$ 
APREMDIX C

Fleld sampling Data 


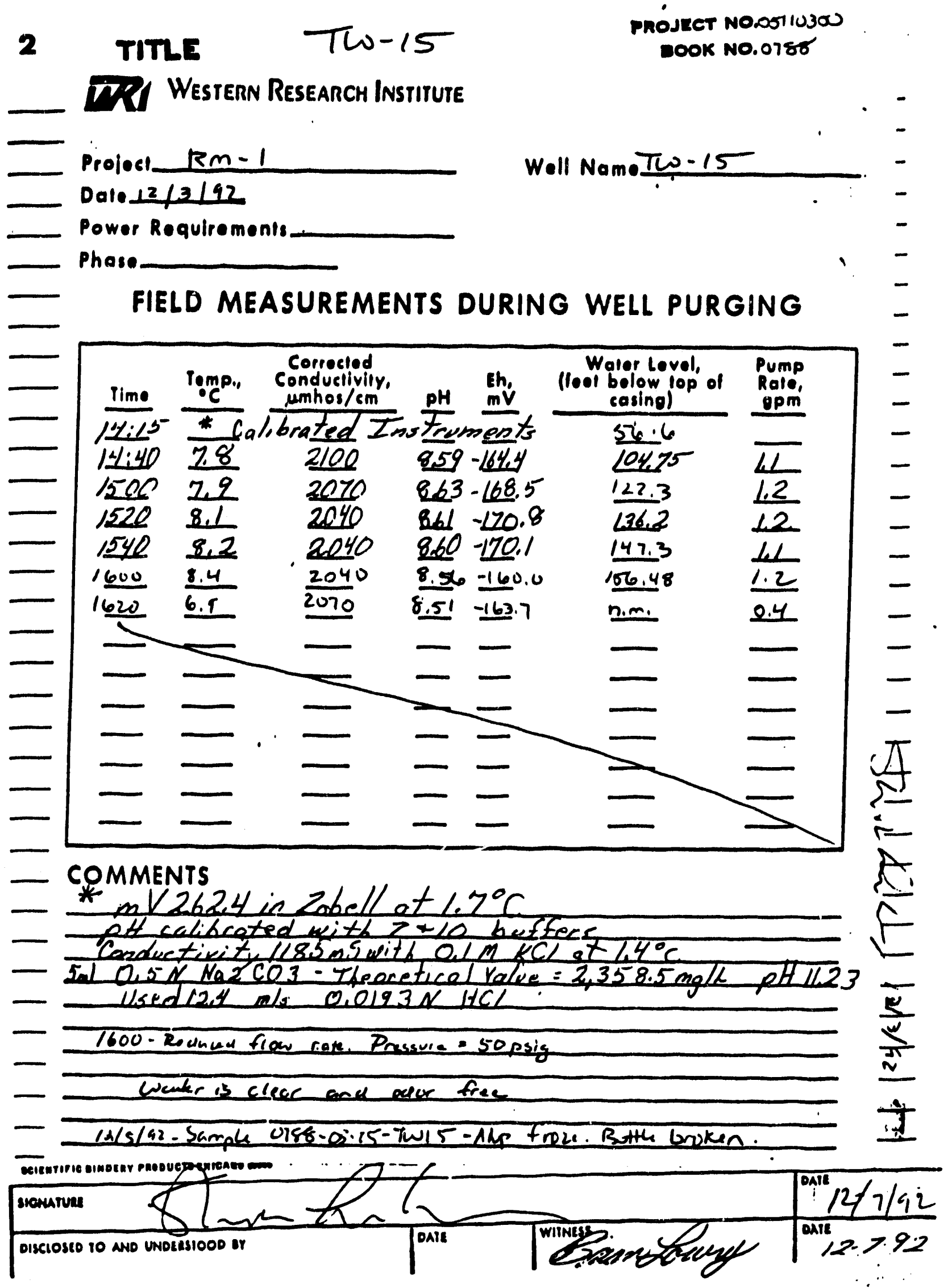


E WRI GROUNDWATER SAMPLING RECORD

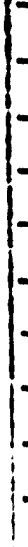

Proiect TLM-1

Date $12 / 3 / 92$

Woather Swartwald

Woll Name Tw-15

Fiold Crow hwebwa Lowry

Air Temperature $\quad 9$

$\left(F^{\circ}\right)$

Measuring Point TOC

Sampling Device Bennett Pmp

Depih to Water Co5.62

Dopth of Samplino Devico $392^{\circ}$

Field Analyses at Time of Sampling

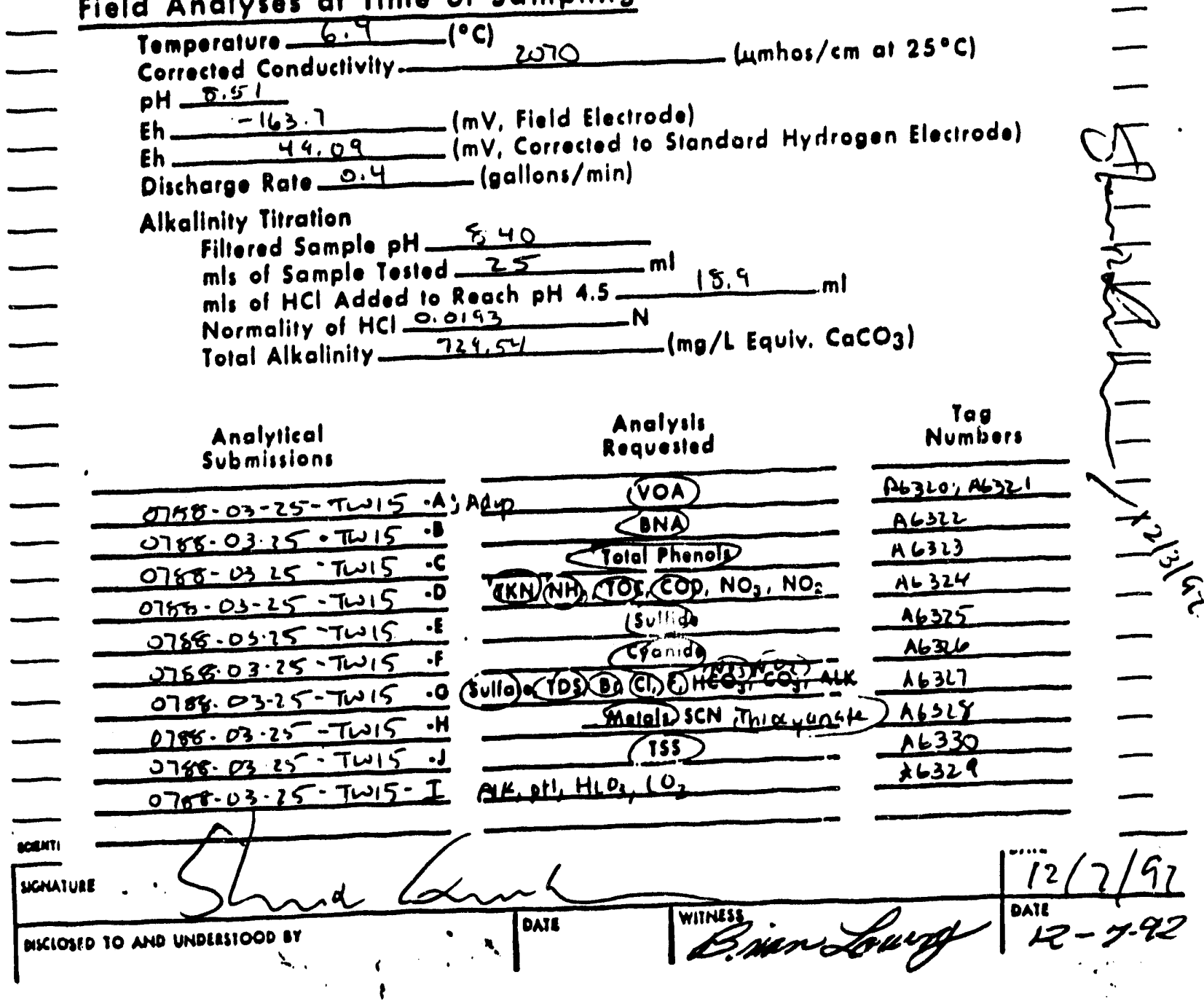




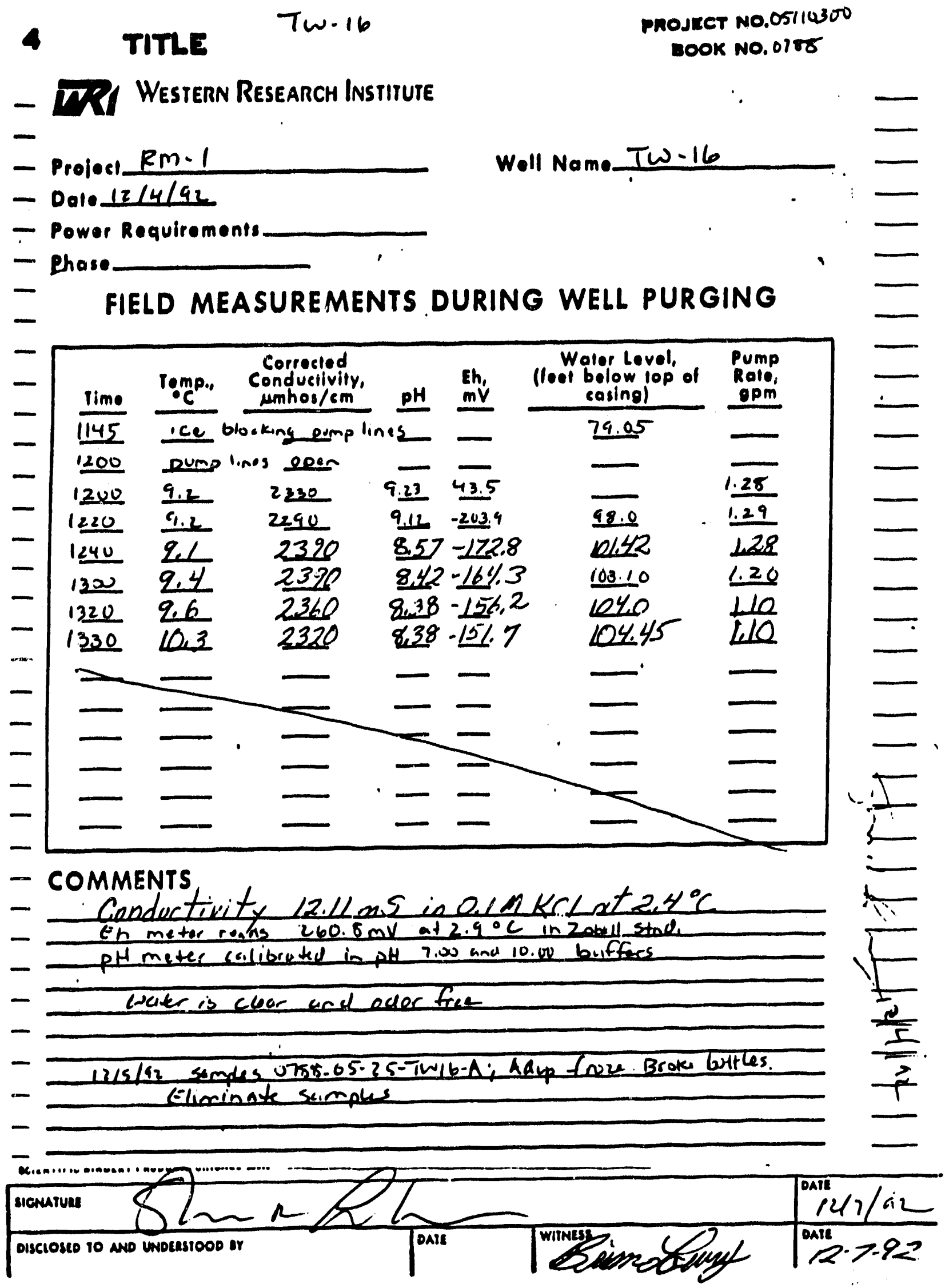




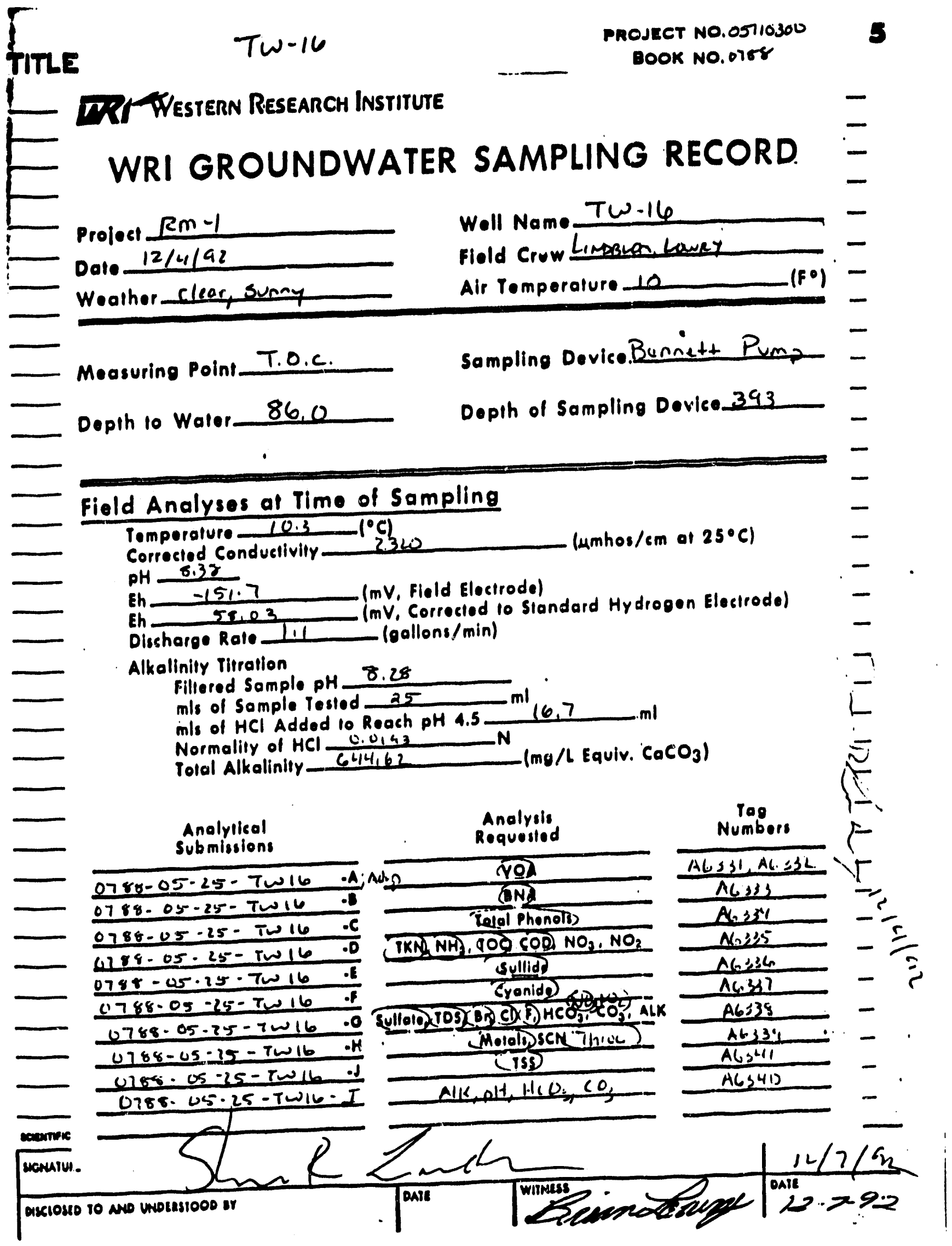




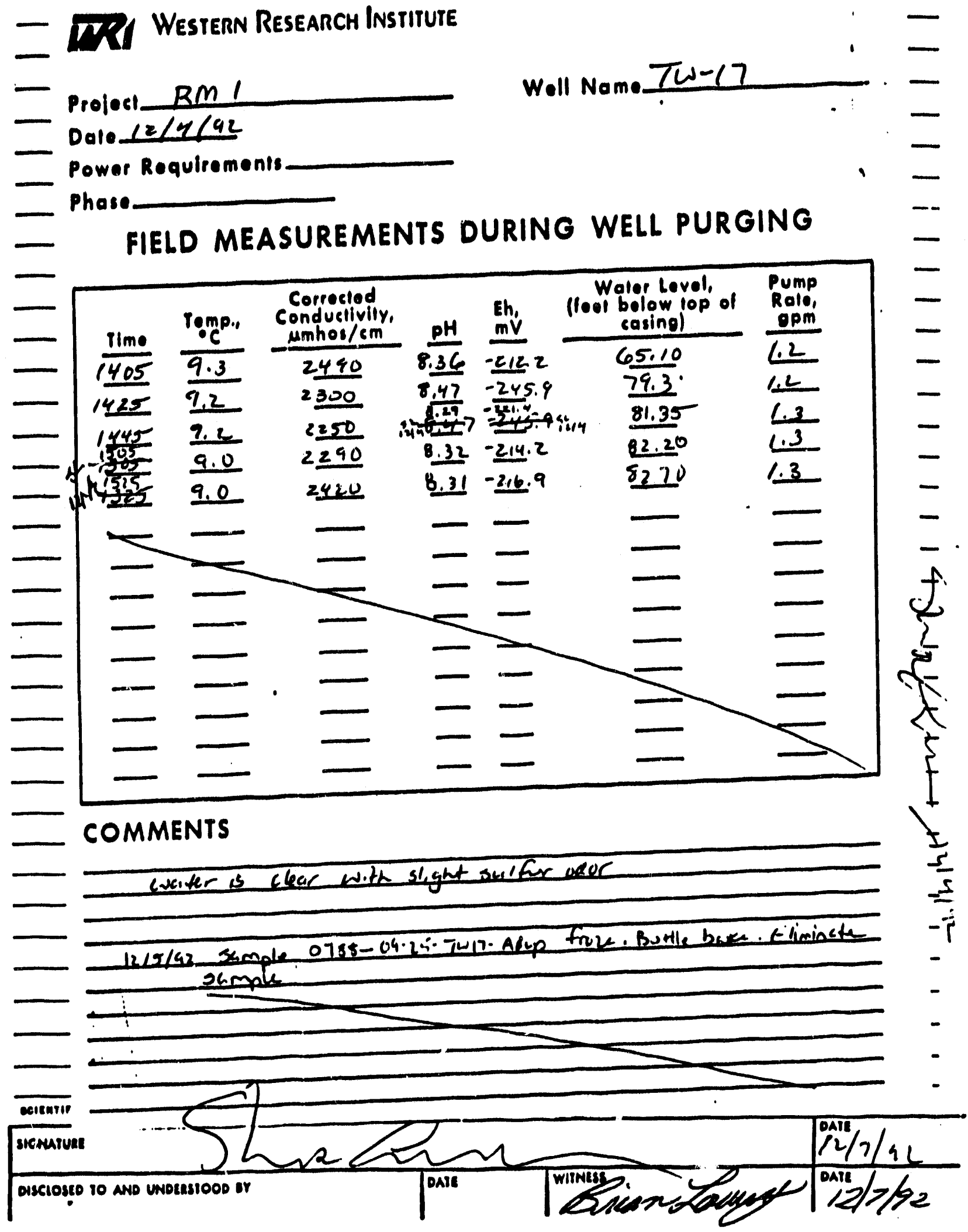




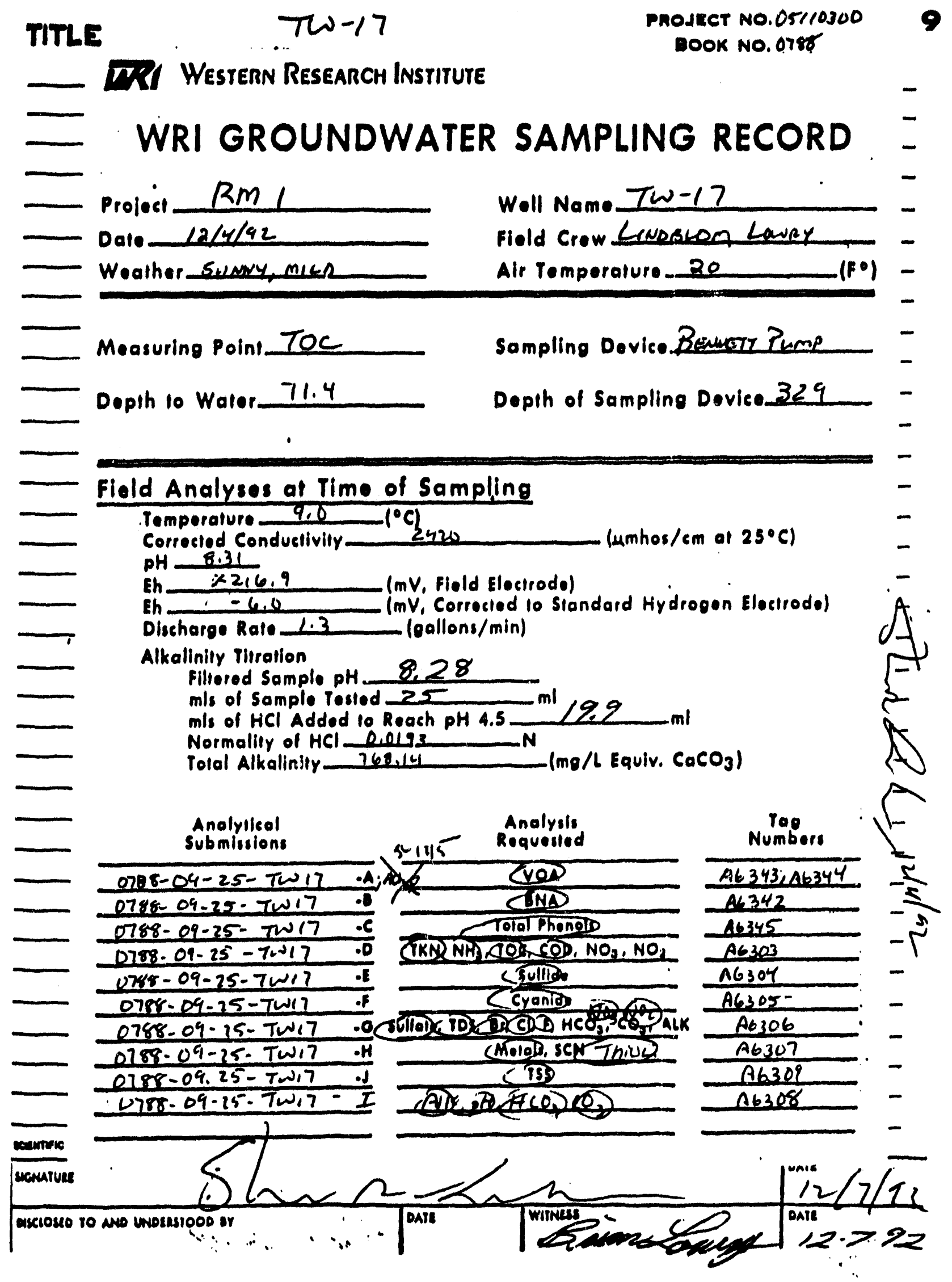


10 TITLE TW-18

PRoject No.0511030

Tíl Western Research Institute

- Project $\frac{R M-1}{12 / 7 / 42}$
- Date $12 /$ Power Requirements-
- Phose

- FIELD MEASUREMENTS DURING WELL PURGING

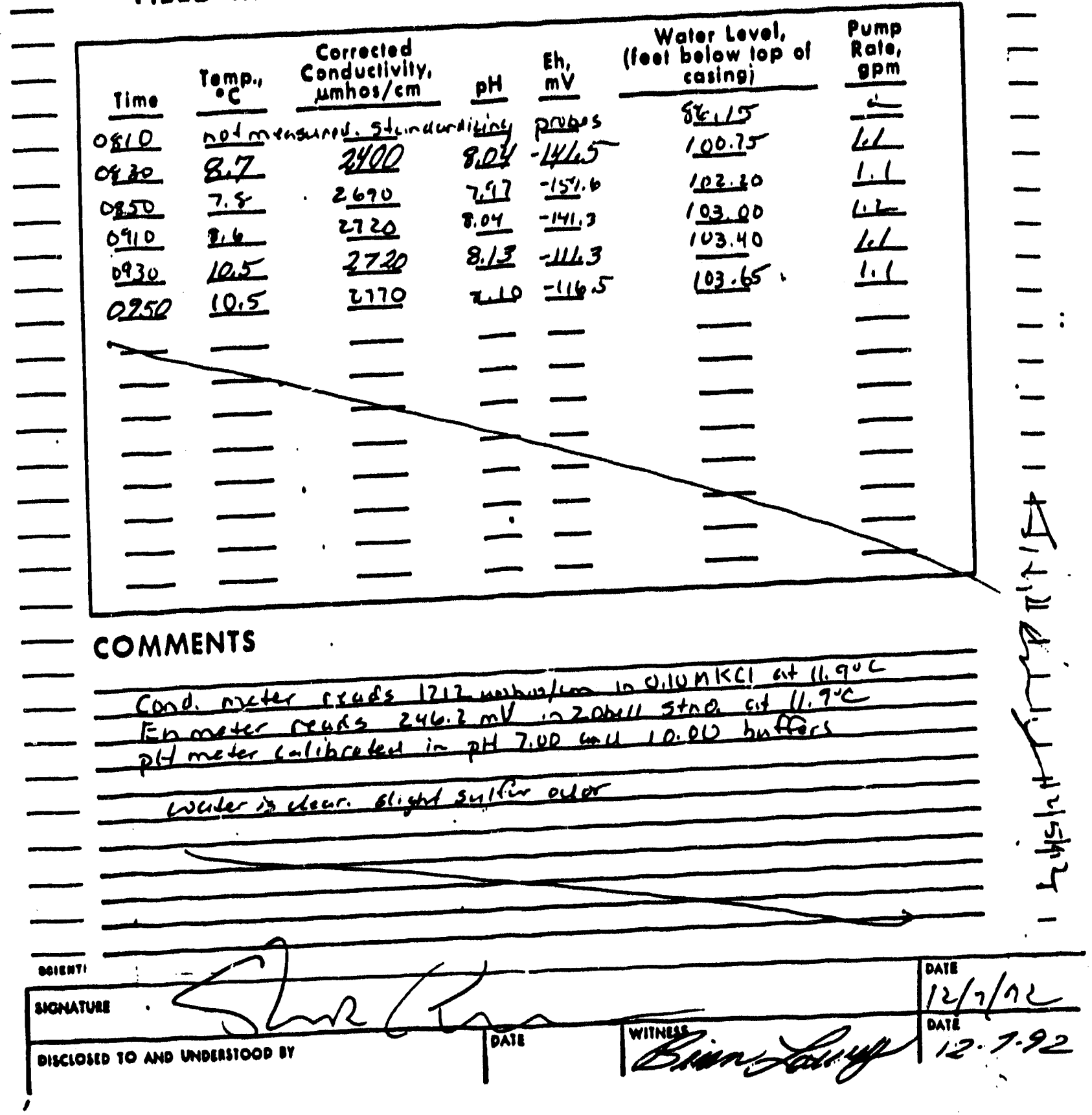




\section{TITLE}

Tw-18

PROJECT NO. 05110300

BOOK NO. 0788

\section{- WRI Western Research Institure}




\section{TITL TW.II}

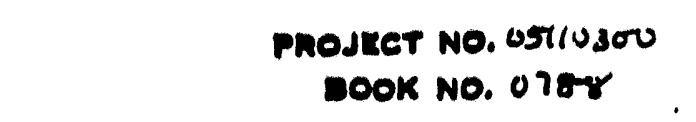

- mill Western Research Institute

-

- Prolocl $R_{M}-1$

- Dale 1217/Gr

-Power Requirements

- Phaso.

- field measurements DURING Well pURGING

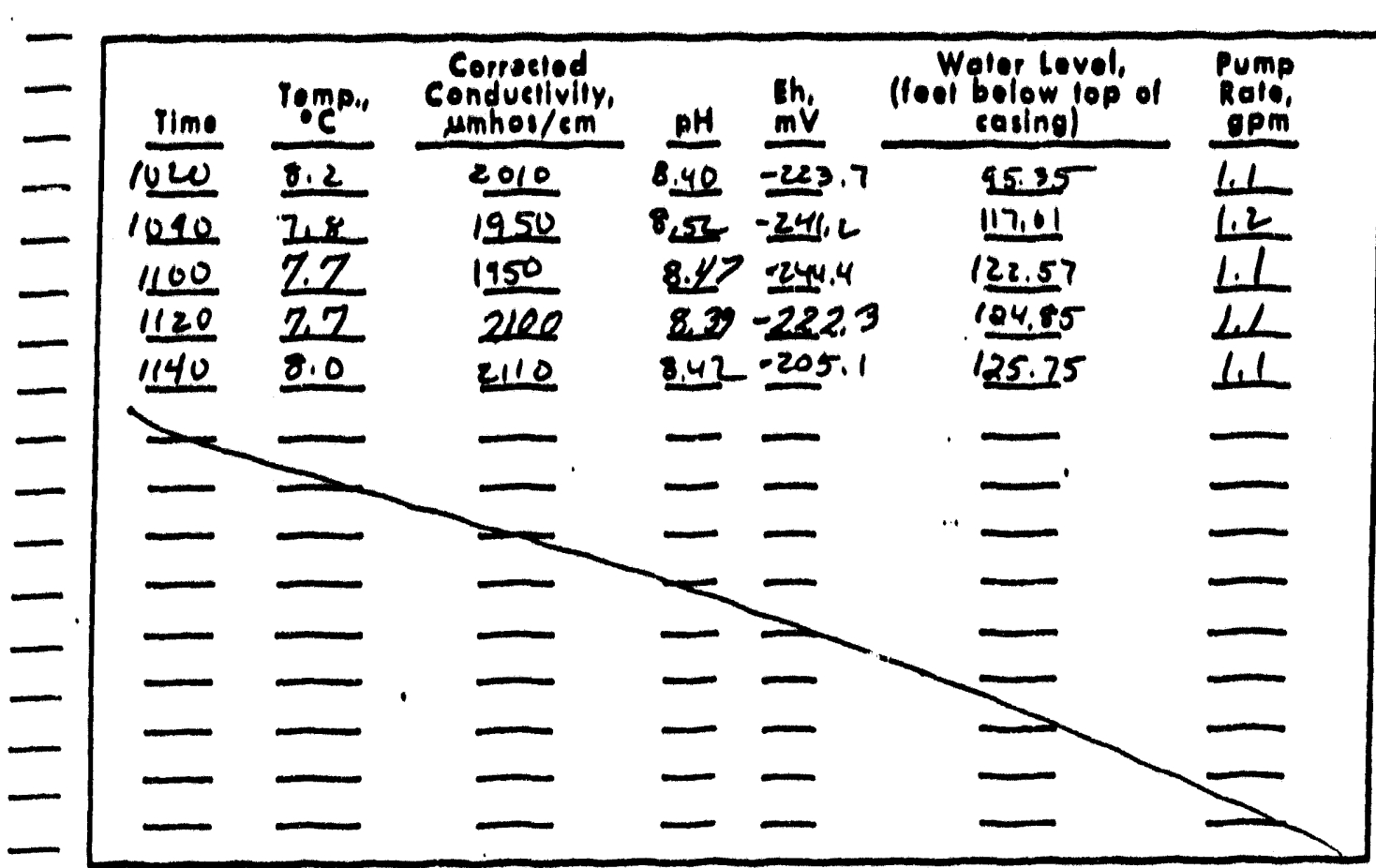

- COMMENTS

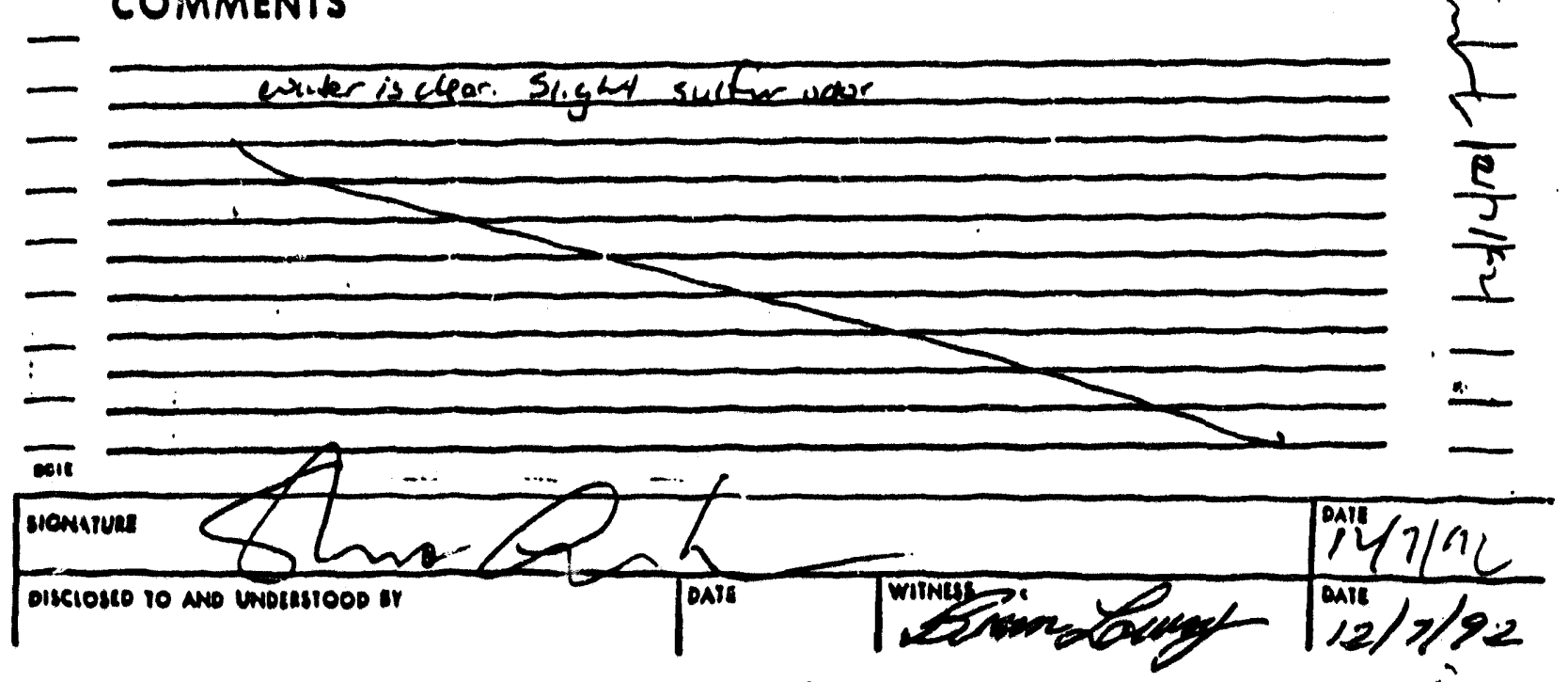




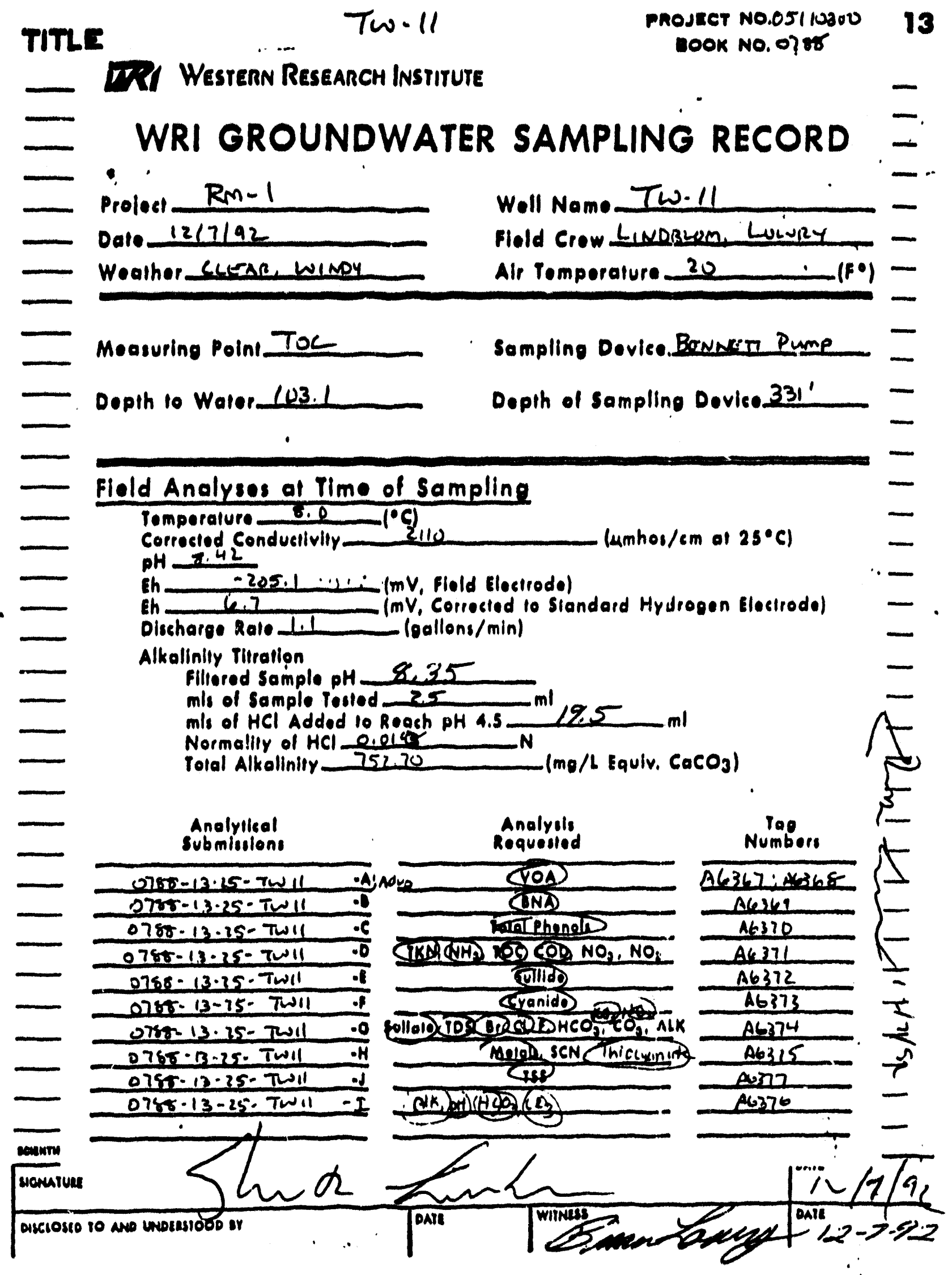




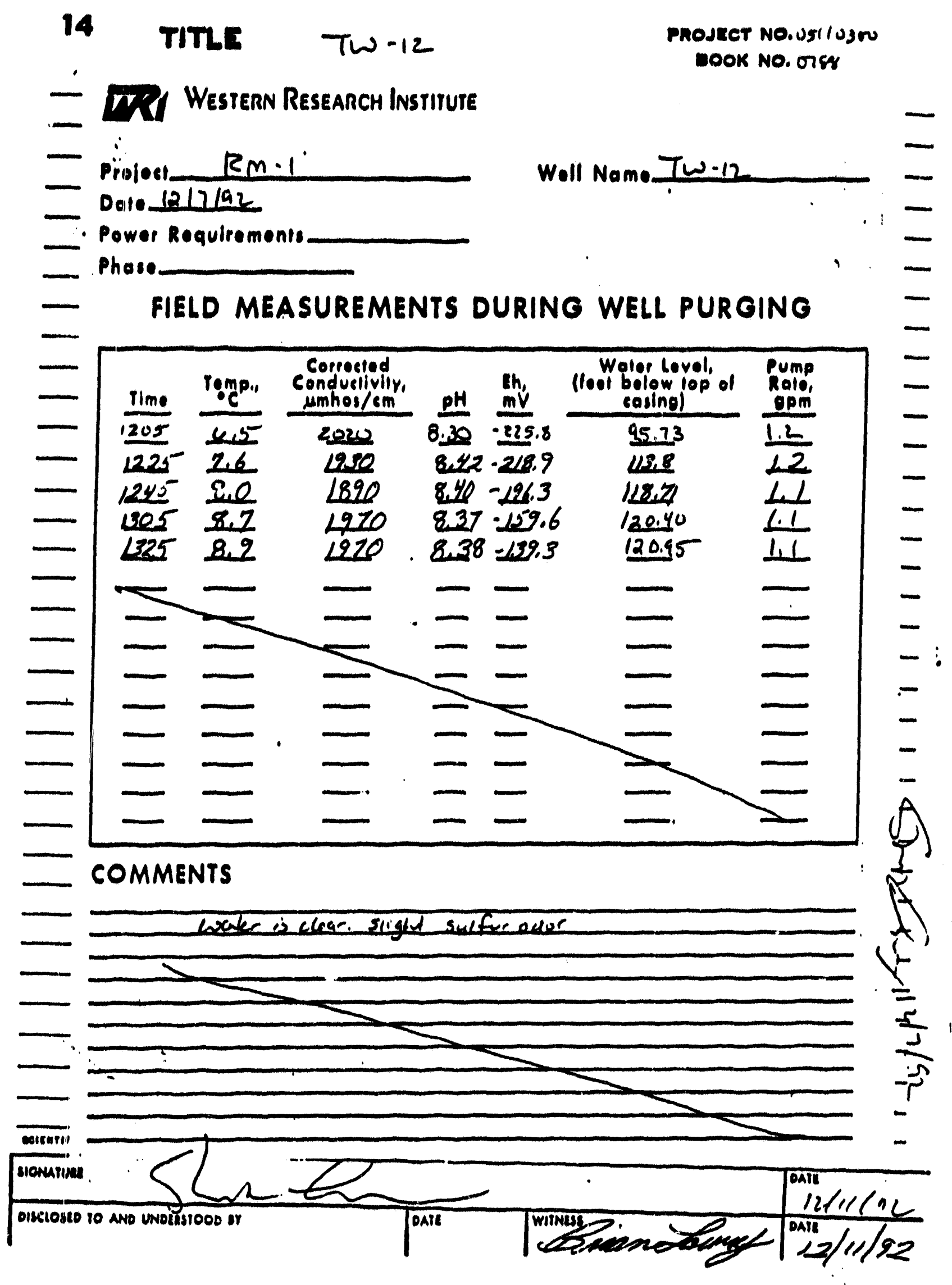




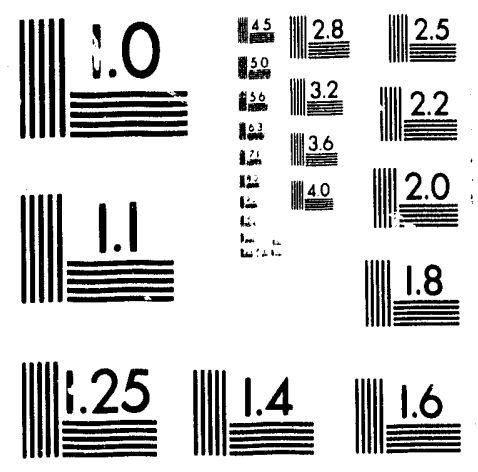



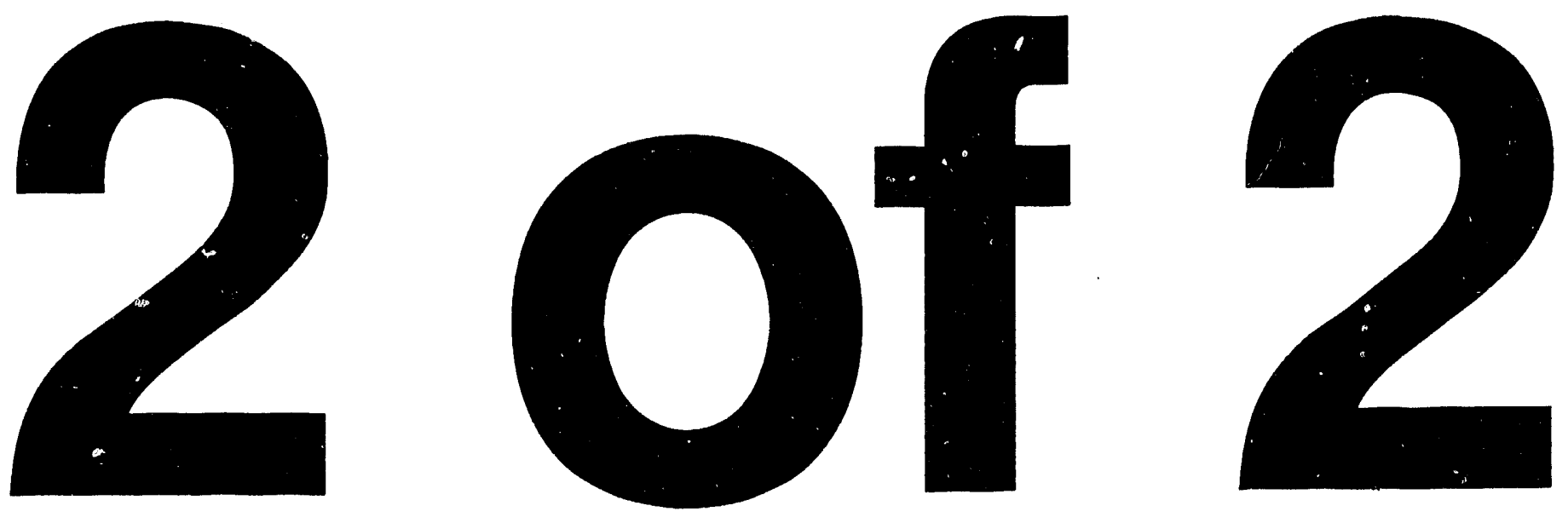


\section{irl Western Research Institute}

\section{$-$}

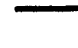

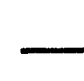

$\longrightarrow$

$\overline{-}$

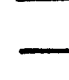

\section{$\equiv$}

.

canming

\section{WRI GROUNDWATER SAMPLING RECORD}

Project $\frac{B m-1}{12 / 7 / 92}$
Dole $\frac{1}{\text { Weather sunm windy }}$

Moasuring Point Tox

Depth to Water 103.51

Woll Name $7 w-12$

Air Temperature 20

Sampling Device Bunneti Pump

'Depth of Sampling Device_344'

Corroctod Conductivity

$\mathrm{PH} \frac{8.38}{-139.3}$

Eh $-\frac{-139.3}{71.69}$ (mV. Fiold Electrode)

Discharge Rate 1.1 (gallons/min)

Alkalinity Titration

Fillered Somple pH 8,33

Fillered Somple pH $\frac{83}{25} \mathrm{ml}$

$\mathrm{m} / \mathrm{s}$ of Sample restod Reach pH $4.5 \mathrm{~m} 18.1 \mathrm{ml}$

Normality of $\mathrm{HCl}-0.0143$

Total Alkalinily

698.64 $N$ img/l Equiv. $\mathrm{CaCO}_{3}$ )

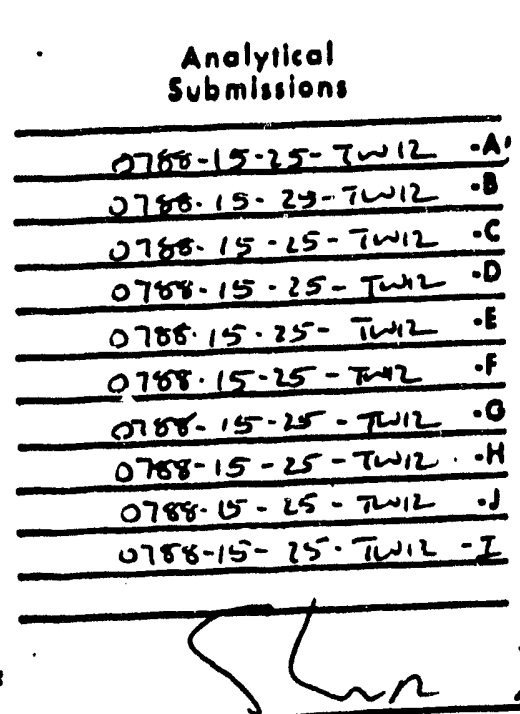

DISCLOSIO TO AND UNDERSIOOD OY
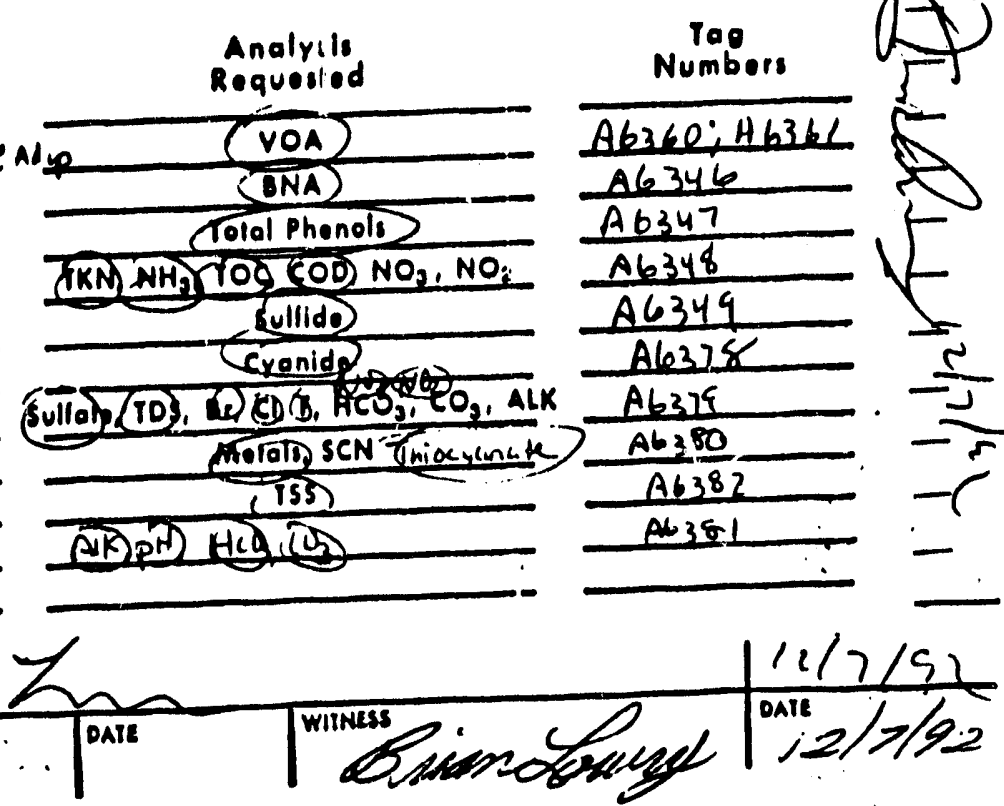


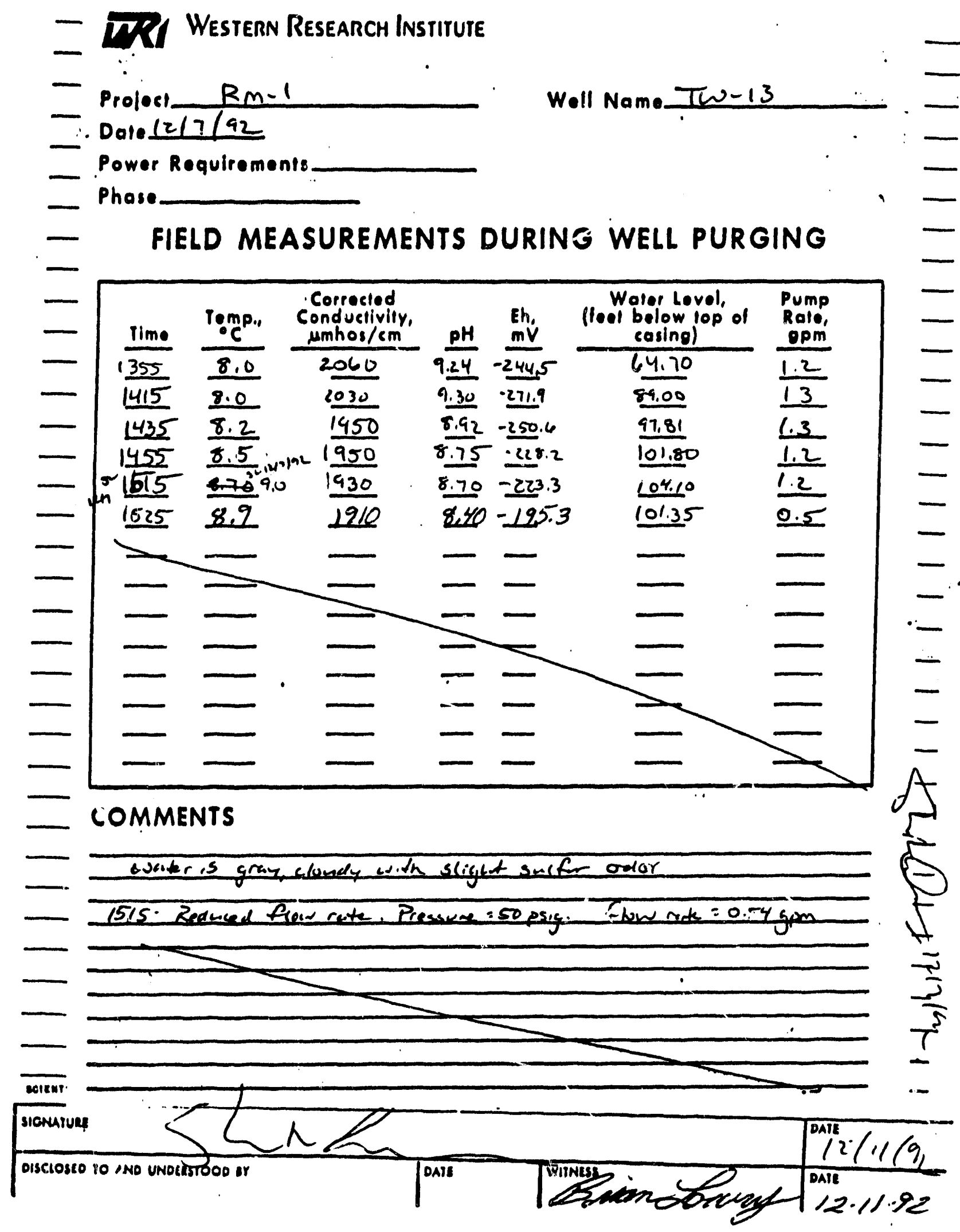




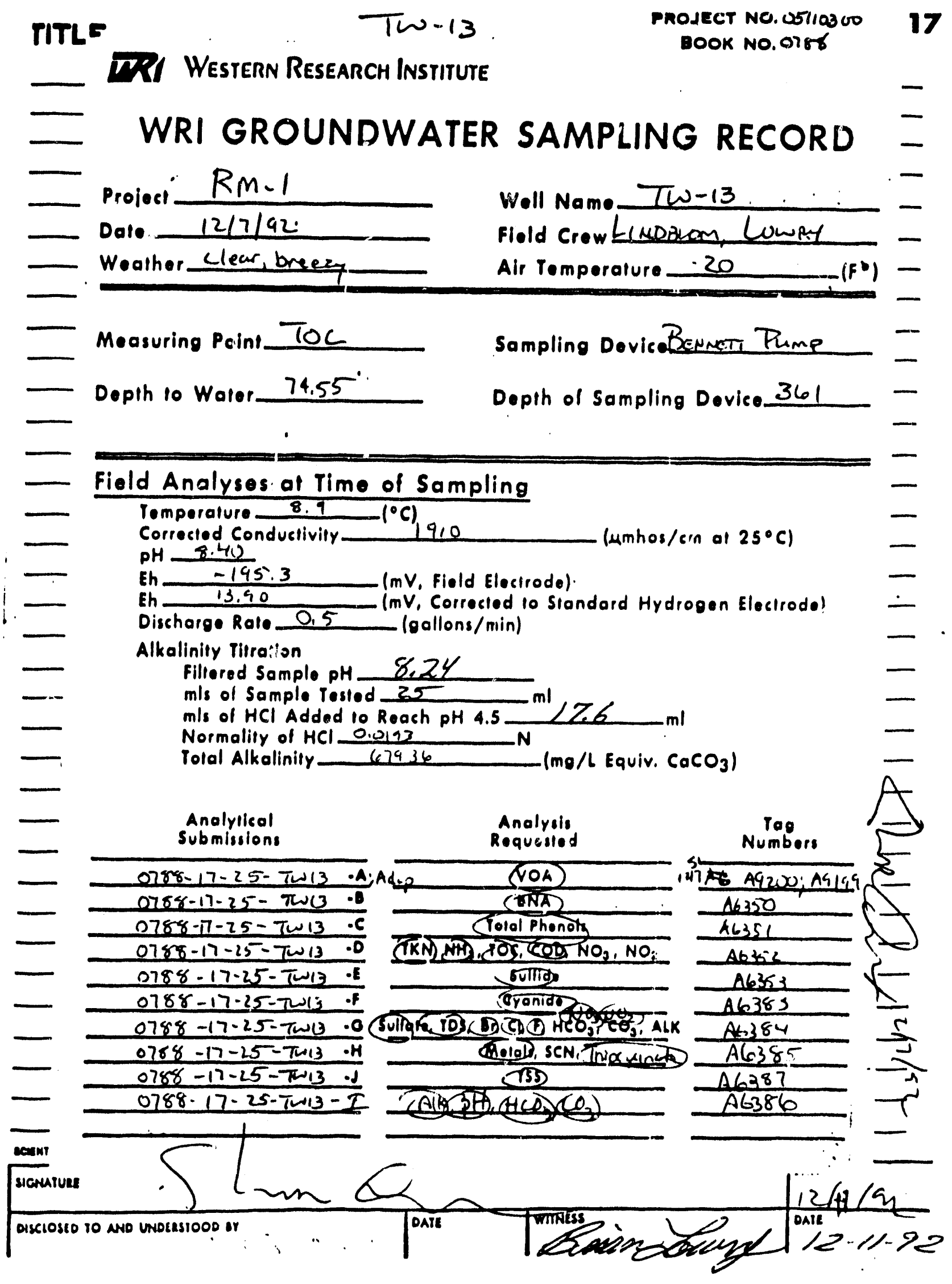




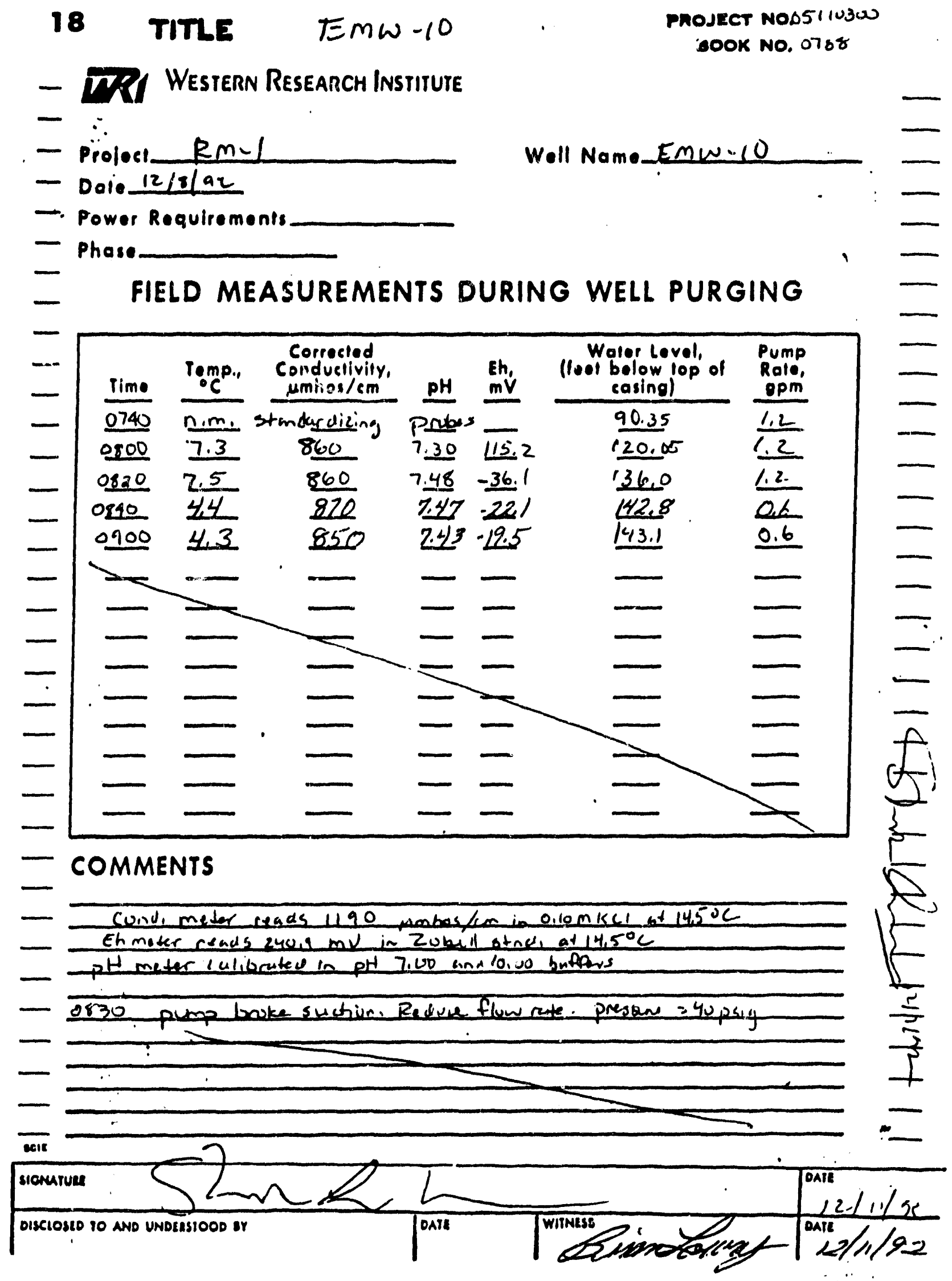




\section{TITLE}

\section{TRI Western Research Institute}

\section{WRI GROUNDWATER SAMPLING RECORD}

Project Rm-1 Well Name Emw-10

Date $12 / 8 / \mathrm{Cl}_{2}$

Weather CWEAR, Winar

Field Crow Linpolom Liwat

Air Temperalure 15

$\left(F^{\circ}\right)$

Measuring Point Toc

Sampling DeviceBLinnotT Pume

Depth to Water. 12.00

Derth of Sampling Device 147

\section{Field Analyses at Time of Sainpling}

Temperature $\left.\frac{4.3}{\text { Corrected Conductivity }}{ }^{\circ} \mathrm{C}\right) 850$ (umhos $/ \mathrm{cm}$ of $25^{\circ} \mathrm{C}$ )

pH. 7.43

Eh -19.5

Eh 195,63 (mV, field Electrode)

Discharge Rate 0.6_ (oallons/min)

Alkalinity Titration

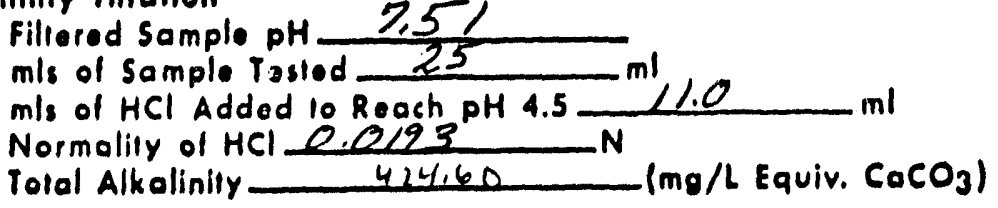

Fillered Sample $\mathrm{pH}$

$\mathrm{mls}$ of Sample Tasted

$\mathrm{m} / \mathrm{s}$ of $\mathrm{HCl}$ Added 10 Reach PH $4.5 \quad \mathrm{~m} / \mathrm{L}$

Normalily of $\mathrm{HCl} \mathrm{O} .0193$

Total Alkolinity

424,60 (mg/l Equiv. $\left.\mathrm{CaCO}_{3}\right)$

Analyilical

Submissions

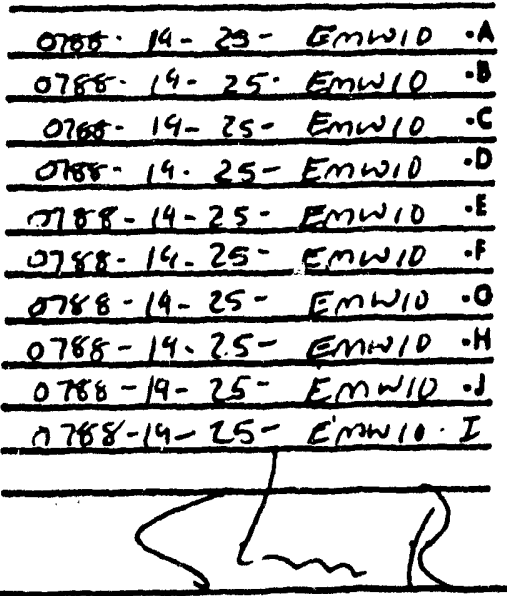

DISCIOSIO 10 ANO UNDERSIOOD OY

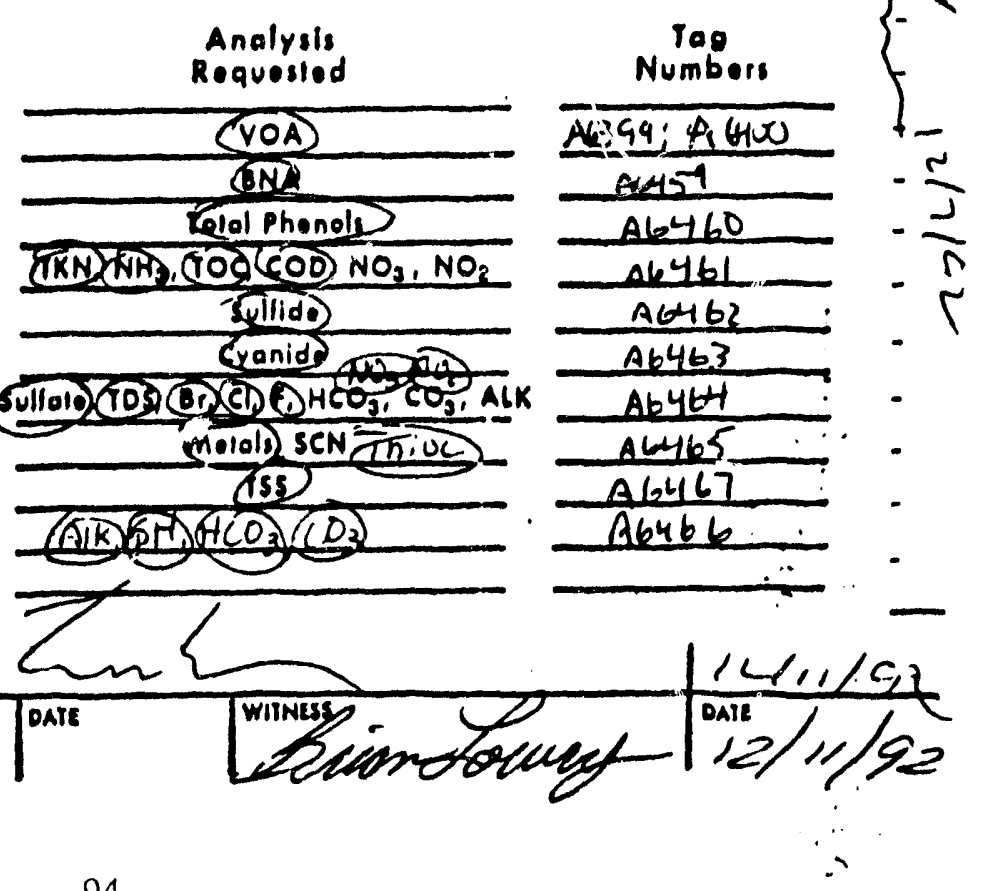

94 
20 TITLE EMW-9

PROLECT NO.05110300

DOOK N0. 0788

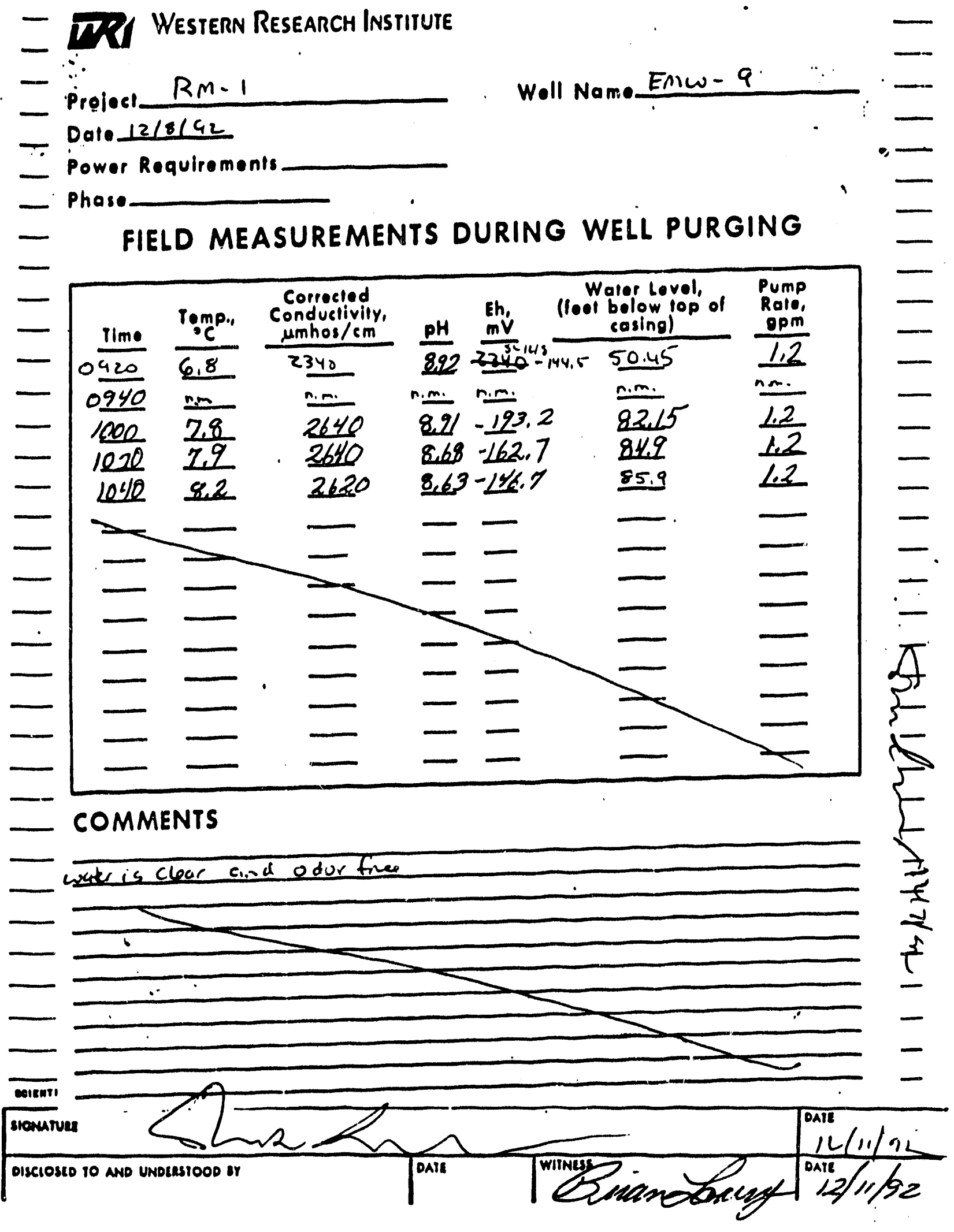




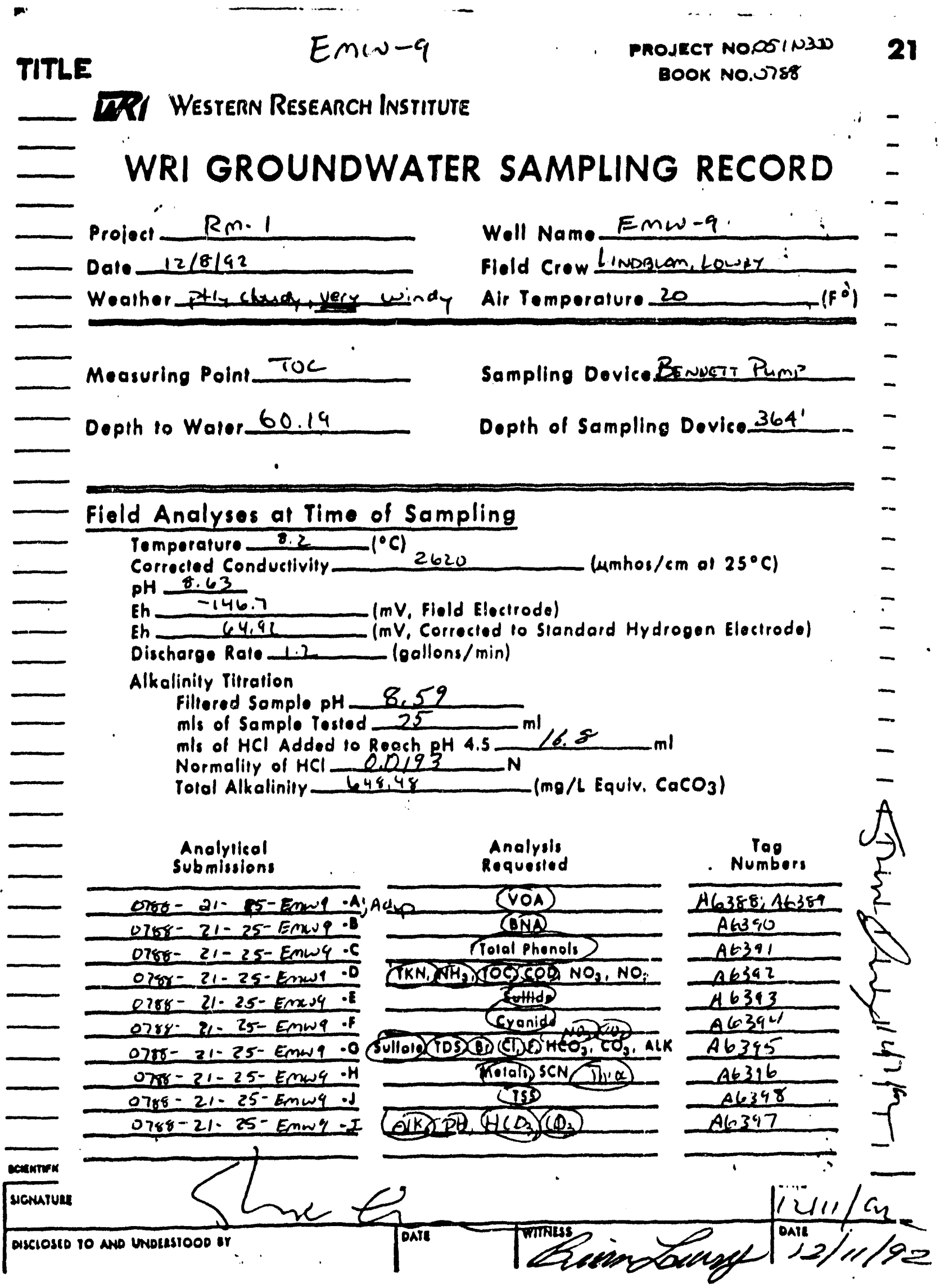




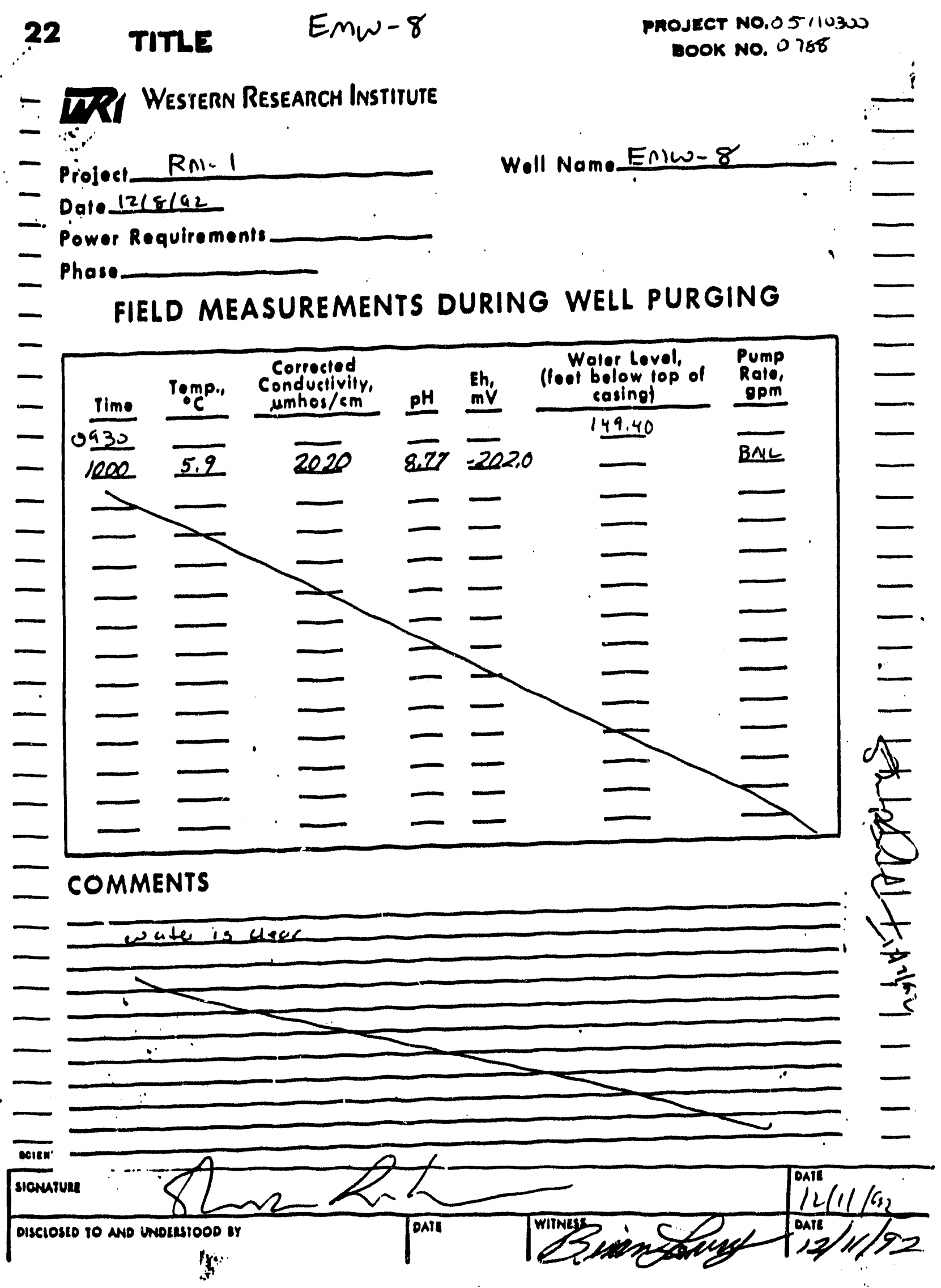




\section{TITLE}

$$
\text { Emw-8 }
$$

PROJECT NOOSINOJON

\section{TrI Western Research Institute}

BOOK NO.O7s8

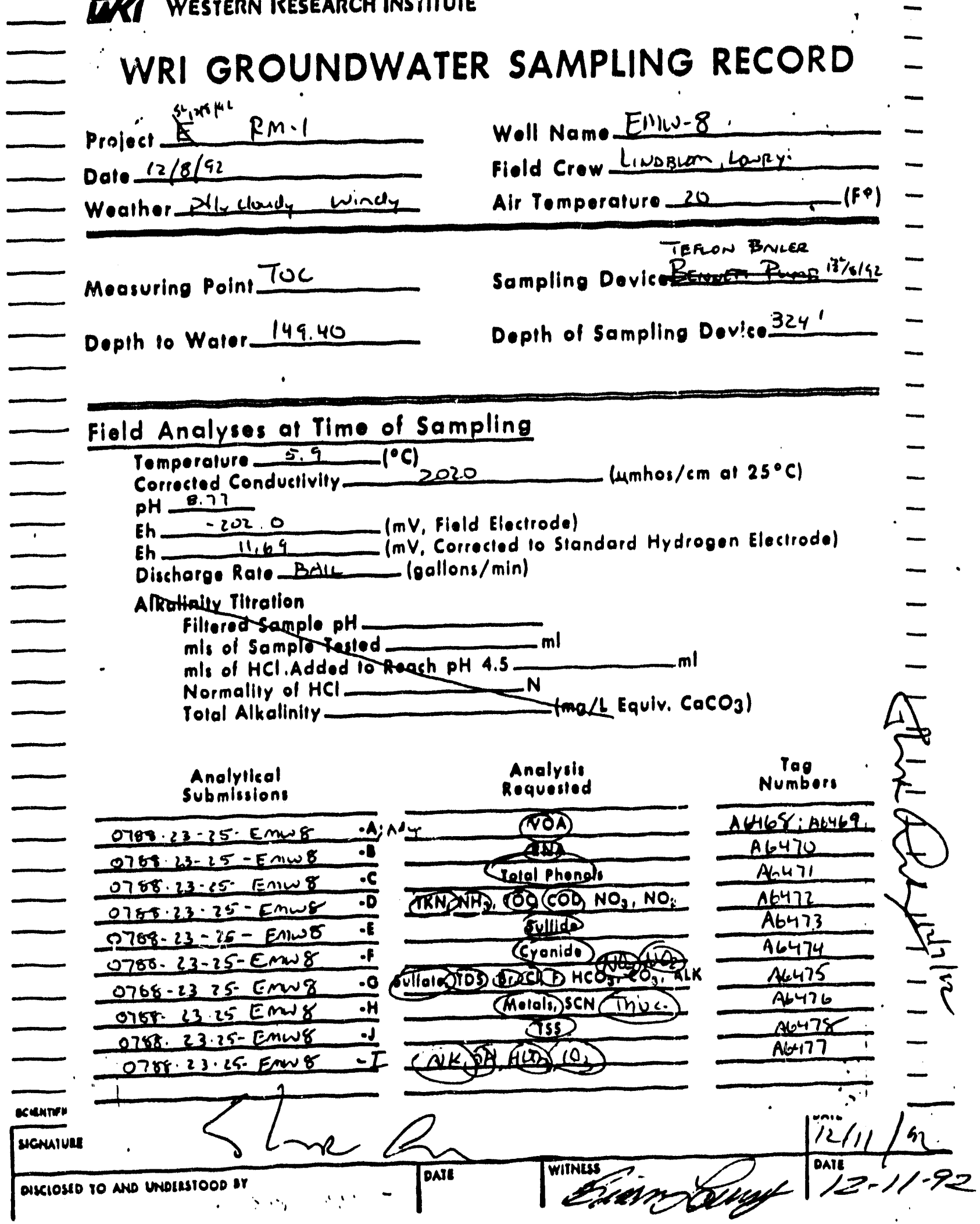




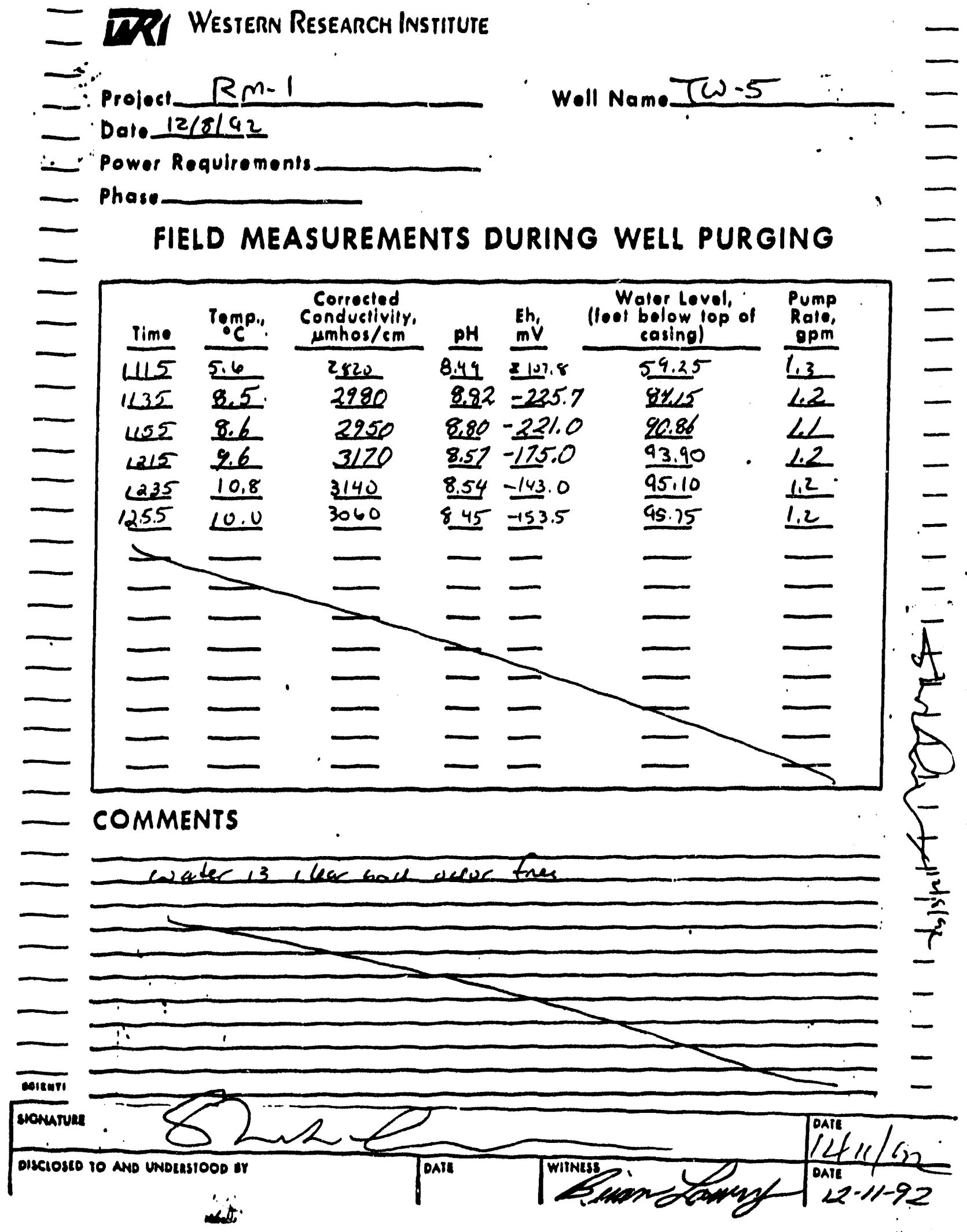


TITLE

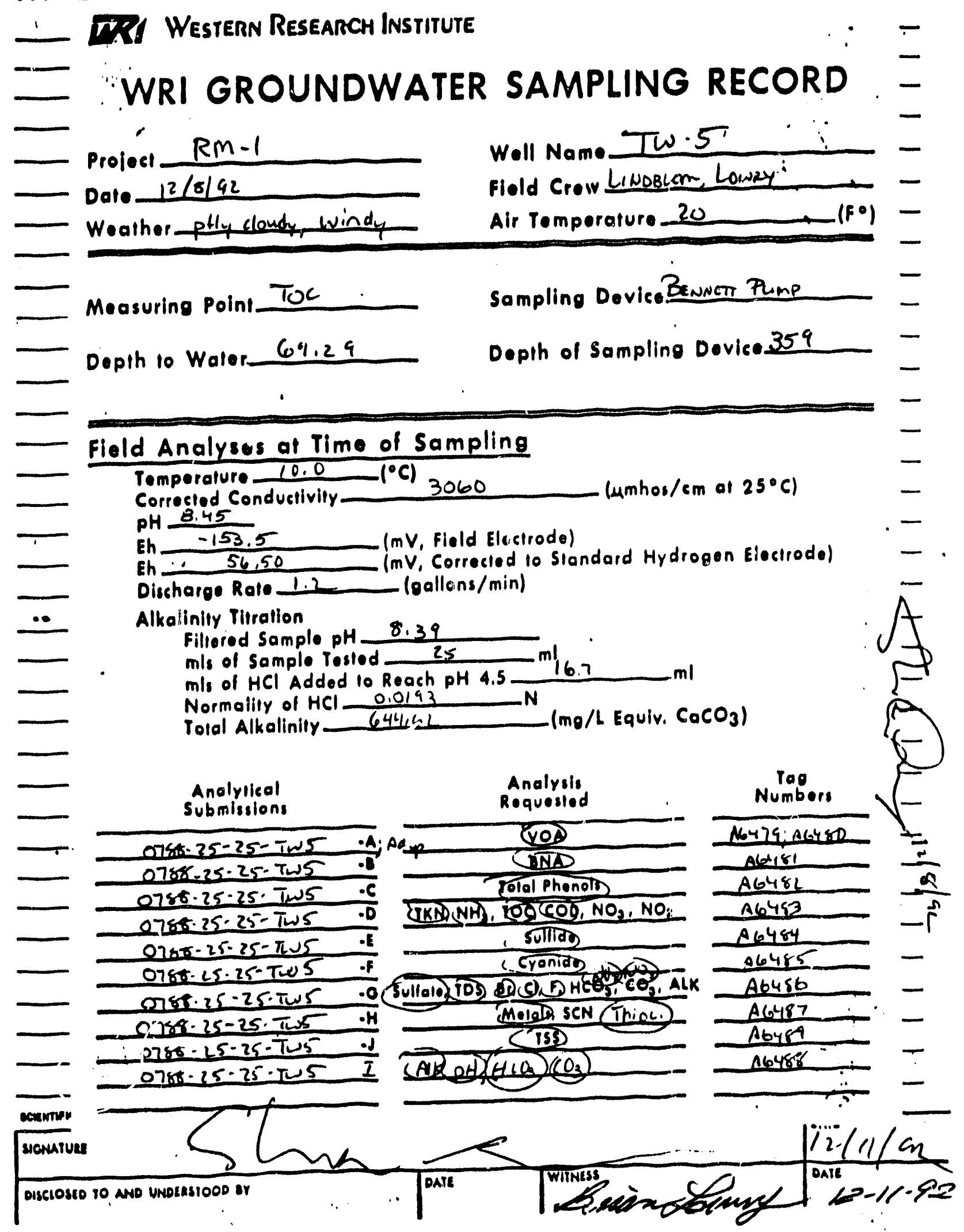




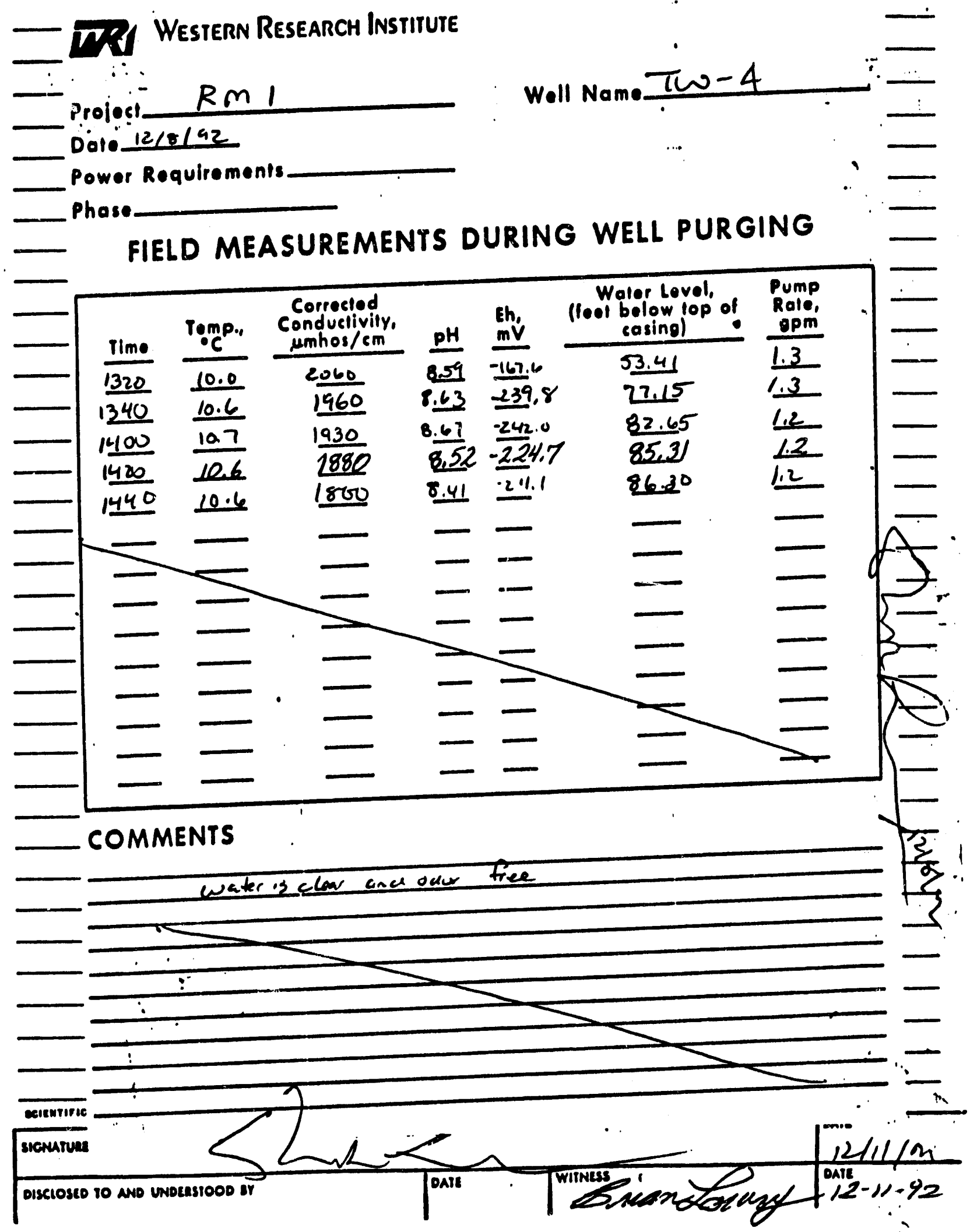




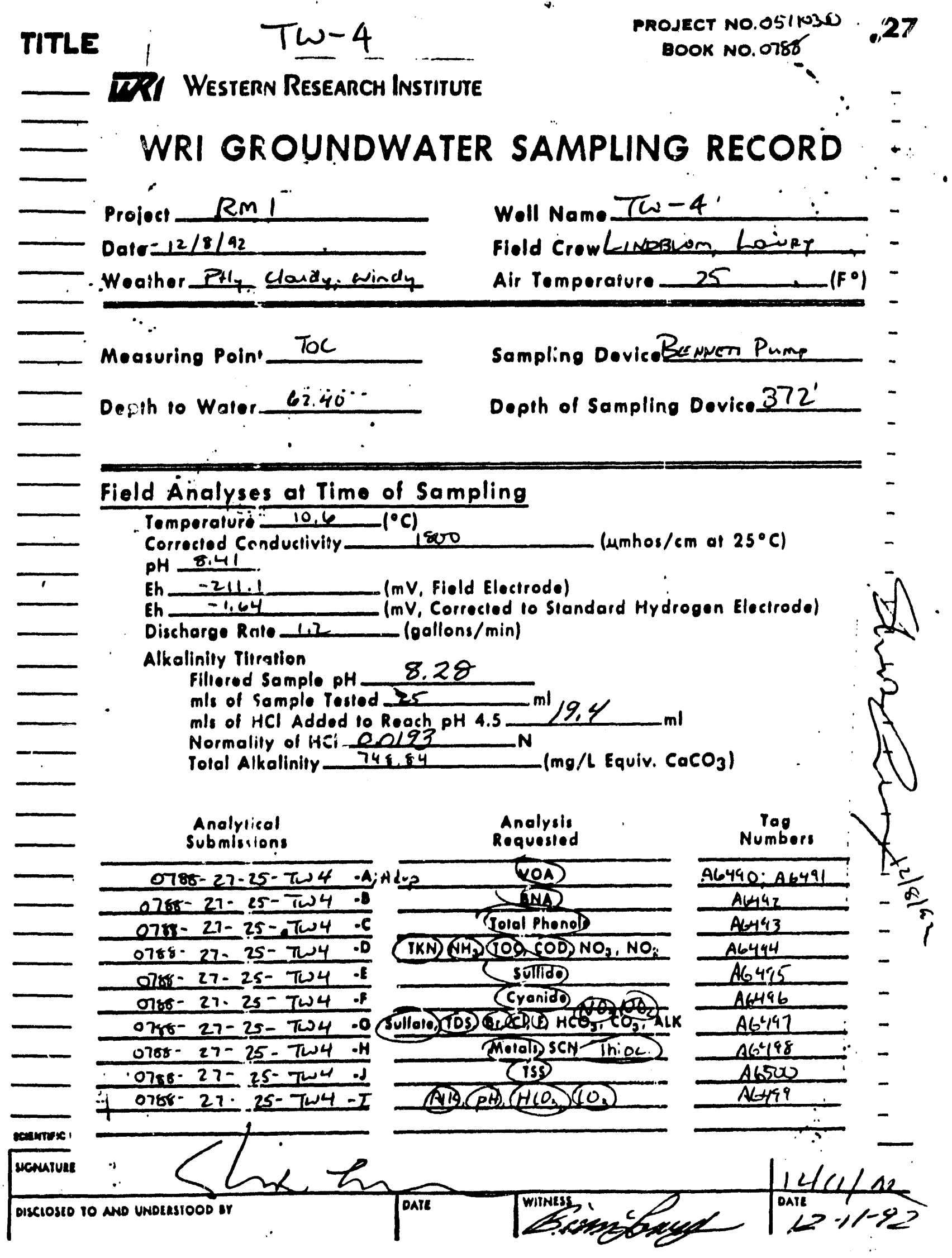




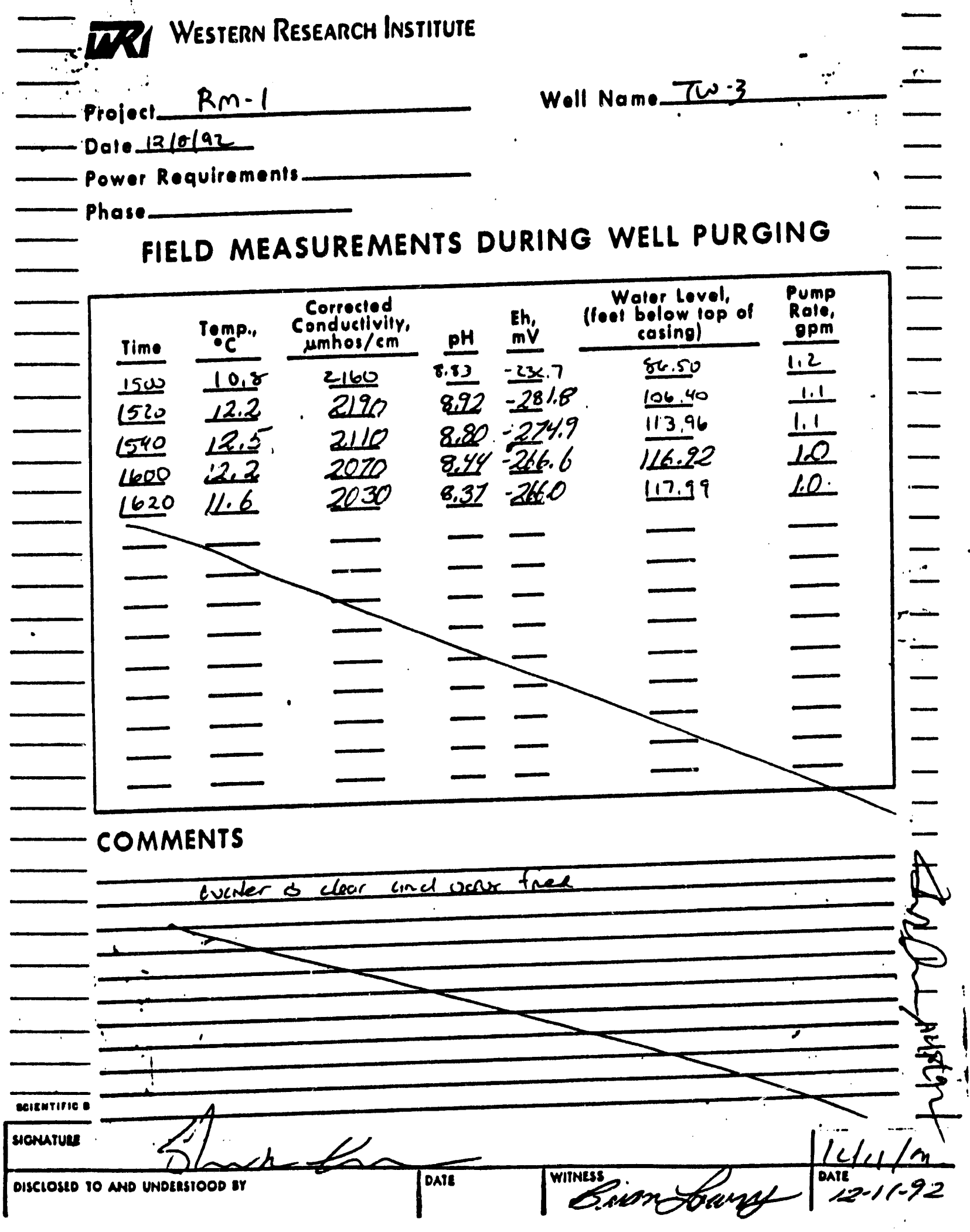




\section{TITLE}

Tw-3

PROJECT NO.05110302

Book No. ors

Fil Western Research Institute

WRI GROUNDWATER SAMPLING RECORD Projoct RMI

Date 12/8/92

Woather ptly doudy, windy

Woll Name Tw-3

field Crow. Linderem Liowiry

Air Tomperature $25,(5 \circ)$

Meosuring Polnt Toc

Sompling Dovice Rennerg Pump

Dopth to Woter 95.05

Depth of Sampling Device 343

Field Analyses at Time of Sampling

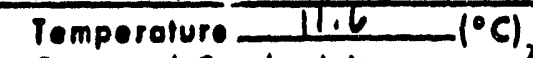

Corrected Conductivily

2030

(umhos $/ \mathrm{cm}$ of $25^{\circ} \mathrm{C}$ )

$\mathrm{PH}+6.37$.

Eh -266.0

- (mV, Field Electrode)

Discharge Rulte 1.0 (gallons/min)

Alkalinily Tilration

Filiorod Sample pH $\frac{8.27}{\text { mls of Sample rosted } \frac{25}{\mathrm{ml}}}$

$\mathrm{mls}$ of $\mathrm{HCl}$ Added 10 Reach $\mathrm{pH} 4.5-\mathrm{ml} 17,9$

Normulity of $\mathrm{HCl} O .0193$

Tolal Aikalinily $\frac{690.94}{6}\left(\mathrm{mg} / \mathrm{L}\right.$ Equiv. $\left.\mathrm{CaCO}_{3}\right)$

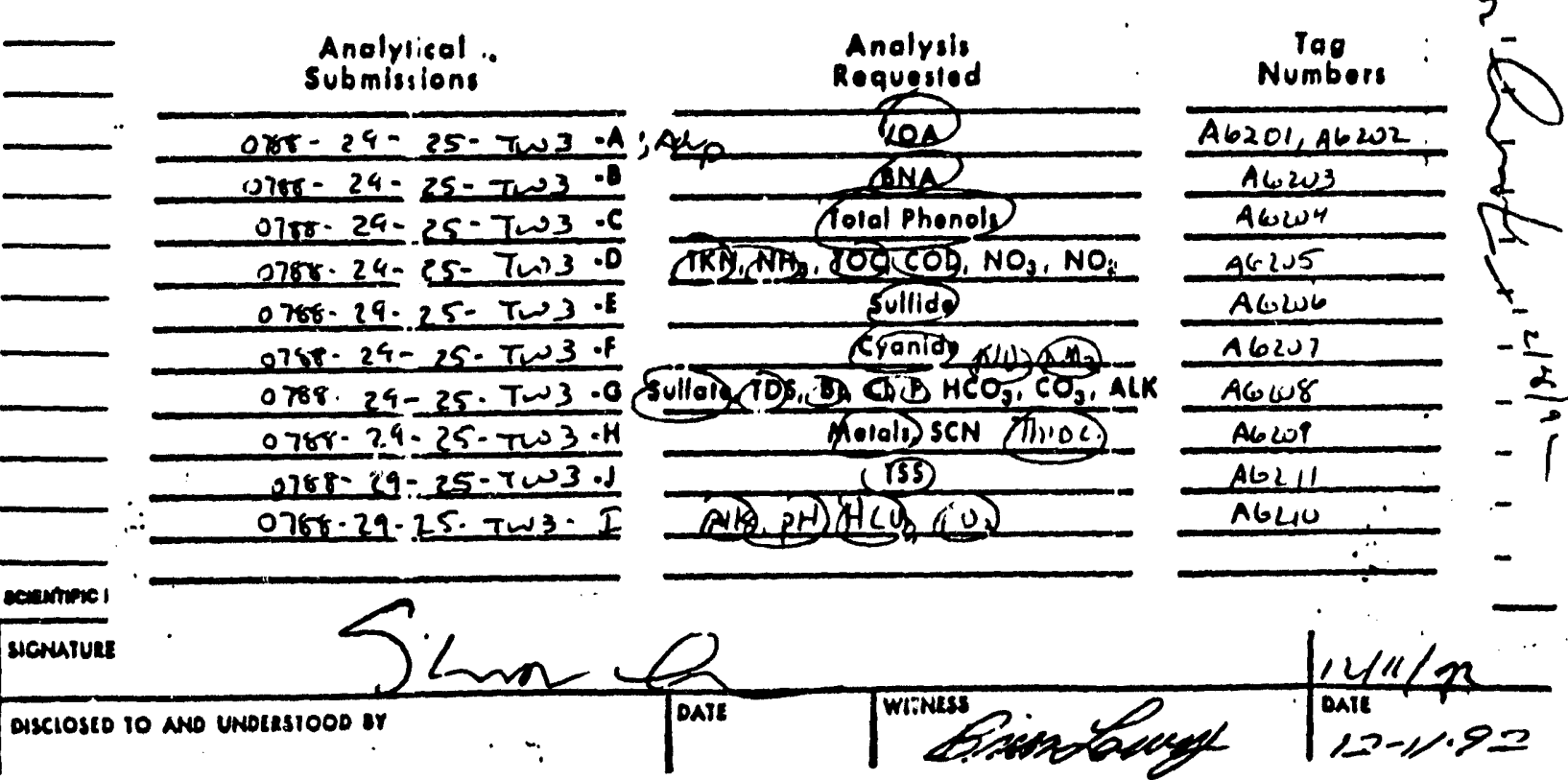




\section{Till Western Research Institute}

Projoci $R m-1$

Date $12 / 9 / 92$

Power Requirements

Phase.

Woll Nome Emw-3

\section{FIELD MEASUREMENTS DURING WELL PURGING}

\begin{tabular}{|c|c|c|c|c|c|c|}
\hline Iime & $\begin{array}{r}\text { romp., } \\
{ }^{2}\end{array}$ & $\begin{array}{l}\text { Correcsed } \\
\text { Conduslivily, } \\
\text { mmhos/cm } \\
\end{array}$ & $\mathrm{pH}$ & $\begin{array}{l}E h_{i} \\
m v\end{array}$ & $\begin{array}{l}\text { Wolor Levol, } \\
\text { (foot bolow top of } \\
\text { casing) }\end{array}$ & $\begin{array}{l}\text { Pump } \\
\text { Rablo, } \\
\text { opm }\end{array}$ \\
\hline 0715 & $n \cdot m$. & stunclundinin & pro & 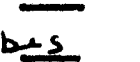 & 95.95 & $=$ \\
\hline$0 \longdiv { 7 3 5 }$ & 11.0 & 2000 & 8.15 & $=249.5$ & 115.81 & $\overline{1.1}$ \\
\hline 0755 & 10,9 & 2040 & 8,25 & -243.5 & $\underline{25.3}$ & 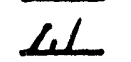 \\
\hline 0815 & 10.6 & 2050 & 8.14 & -232.4 & 228.4 & 10 \\
\hline 0835 & 10.6 & 2060 & 8.11 & -229.9 & 129.15 & 1.1 \\
\hline 0845 & 9.6 & 2180 & 8.9 & -235.6 & 128.15 & 0.75 \\
\hline & & - & - & 一 & $=$ & - \\
\hline & & & & - & - & - \\
\hline & & & & & & - \\
\hline & & & - & - & & - \\
\hline & & & - & - & & \\
\hline
\end{tabular}

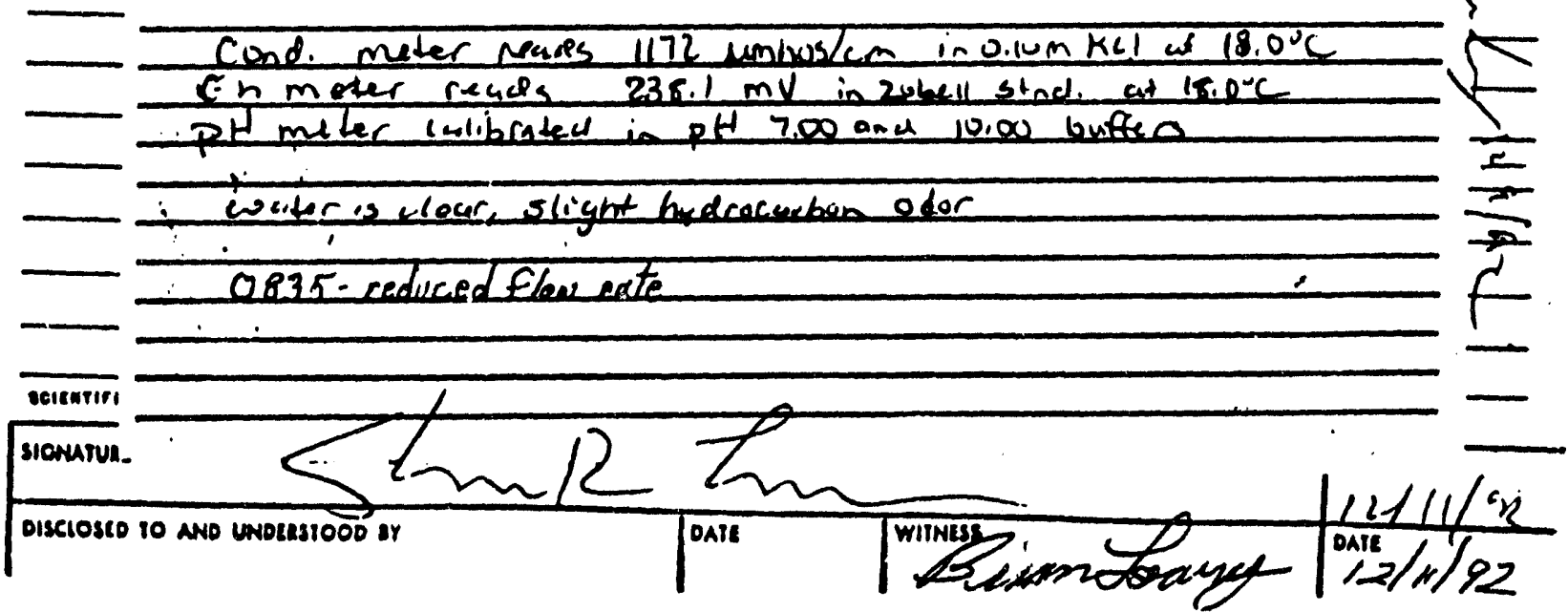




\section{TITLE}

Emw-3

PROJECT NO. OOIIU30

BOOK No. OT88

Fi! Western Research Institute

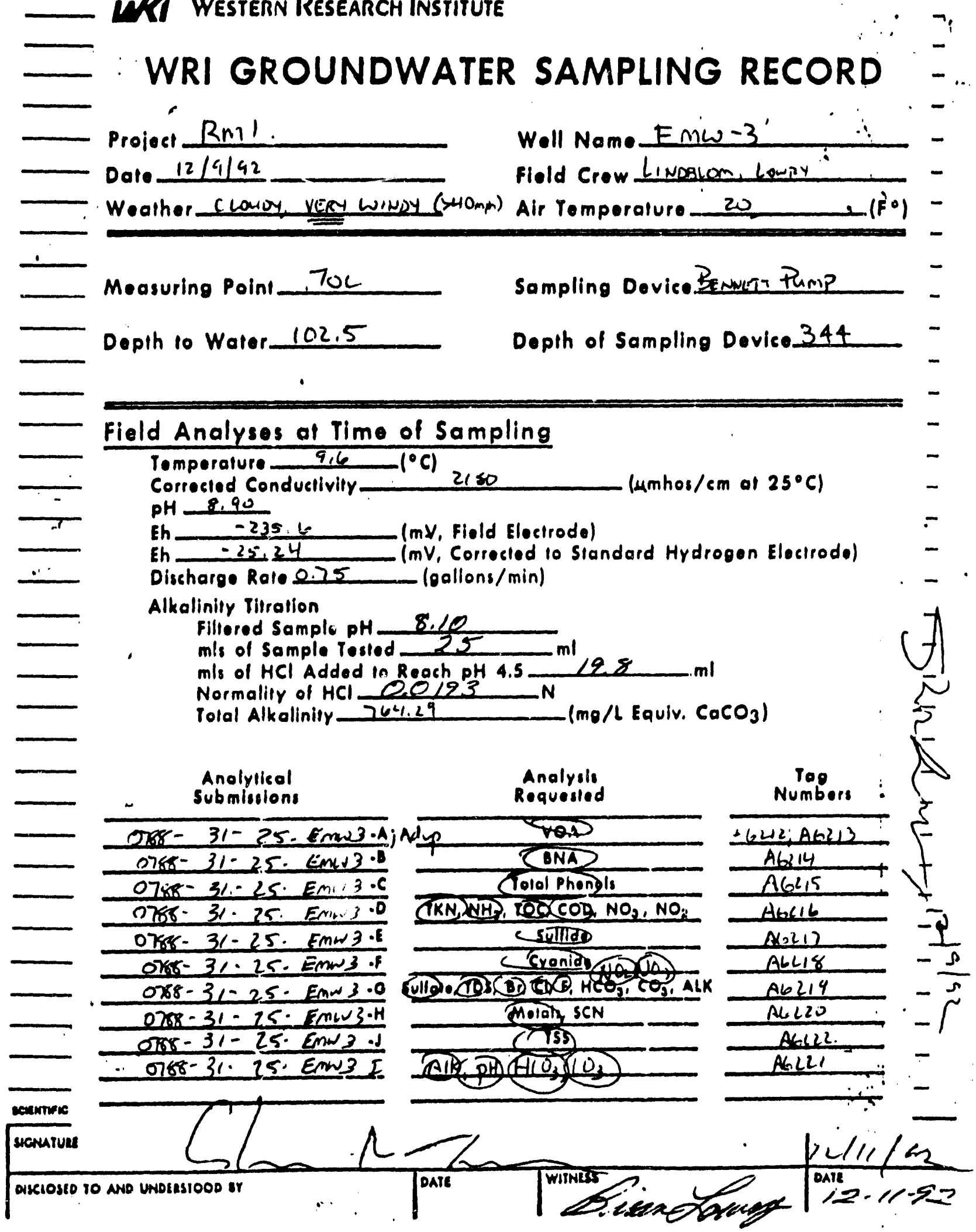


32 TITLE TW-2

PROJECT NO.0511030

BOOK NO. 0788

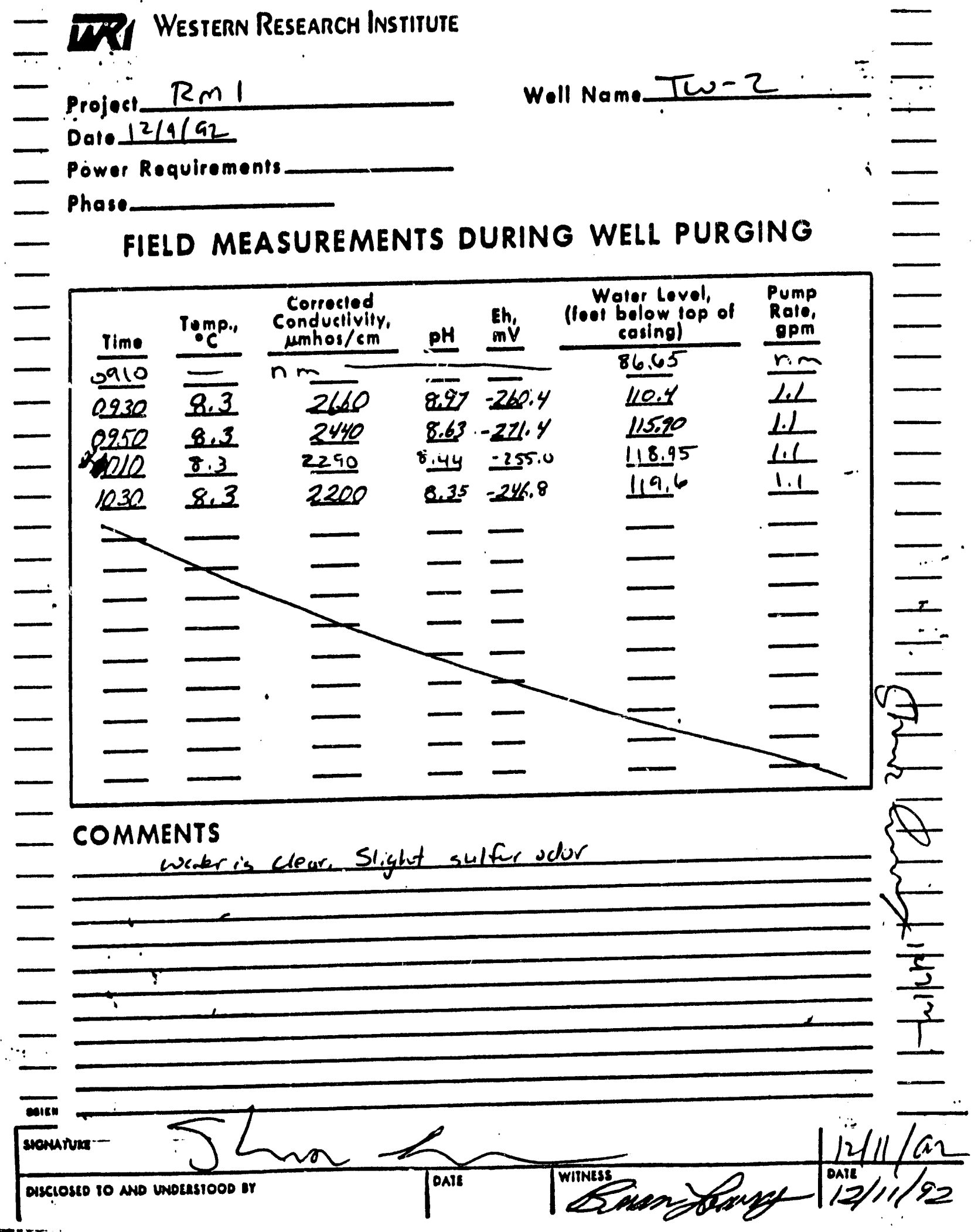




\section{TITLE Tw- 2}

- TRI Western Research Institute

BOOK :10.0?85

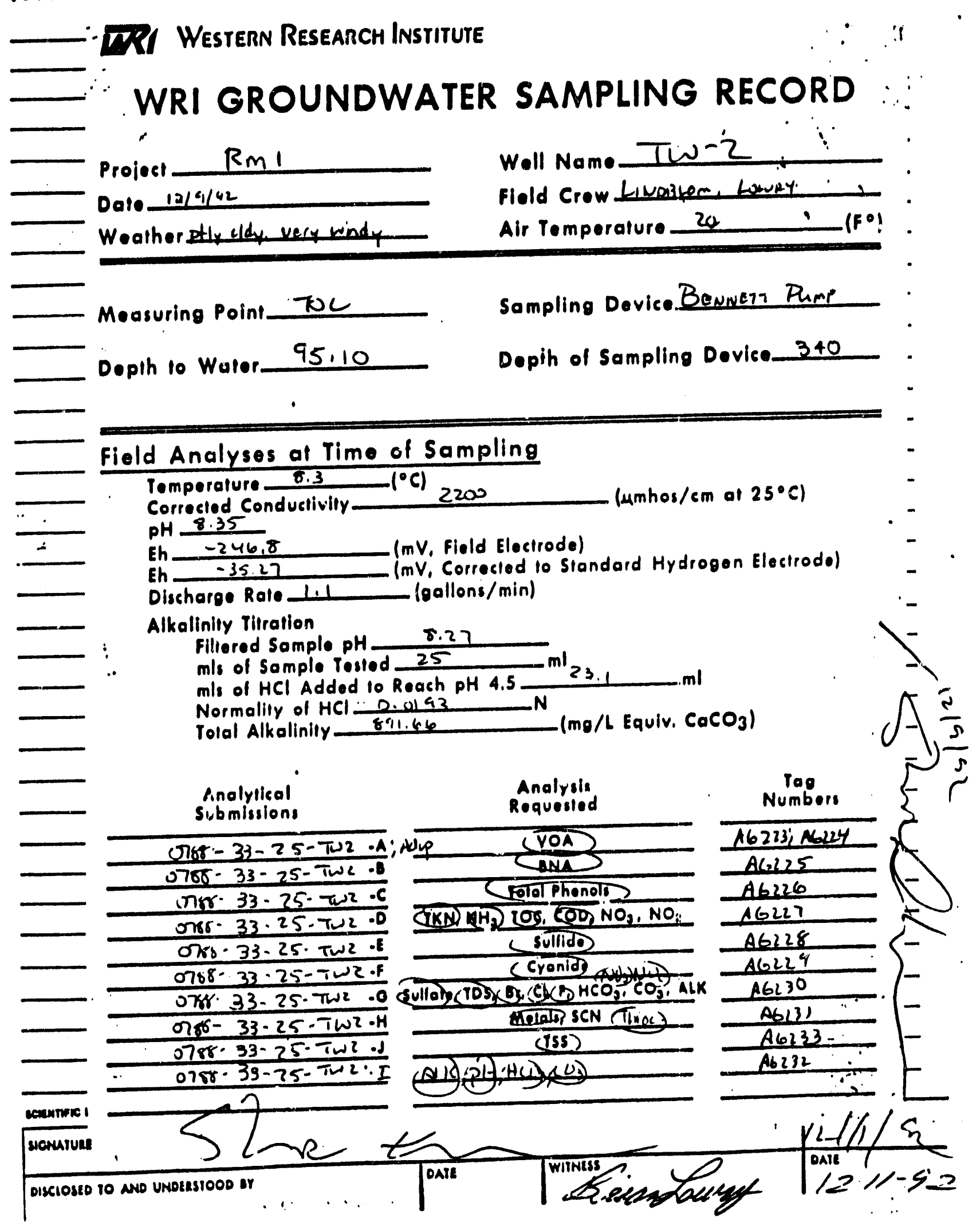




\section{Till Western Research Institute}

$\therefore$

Project

$R m 1$

Date $12 / 9 / 92$

Power Requirements

Phase-

FIELD MEASUREMENTS DURING WELL PURGING

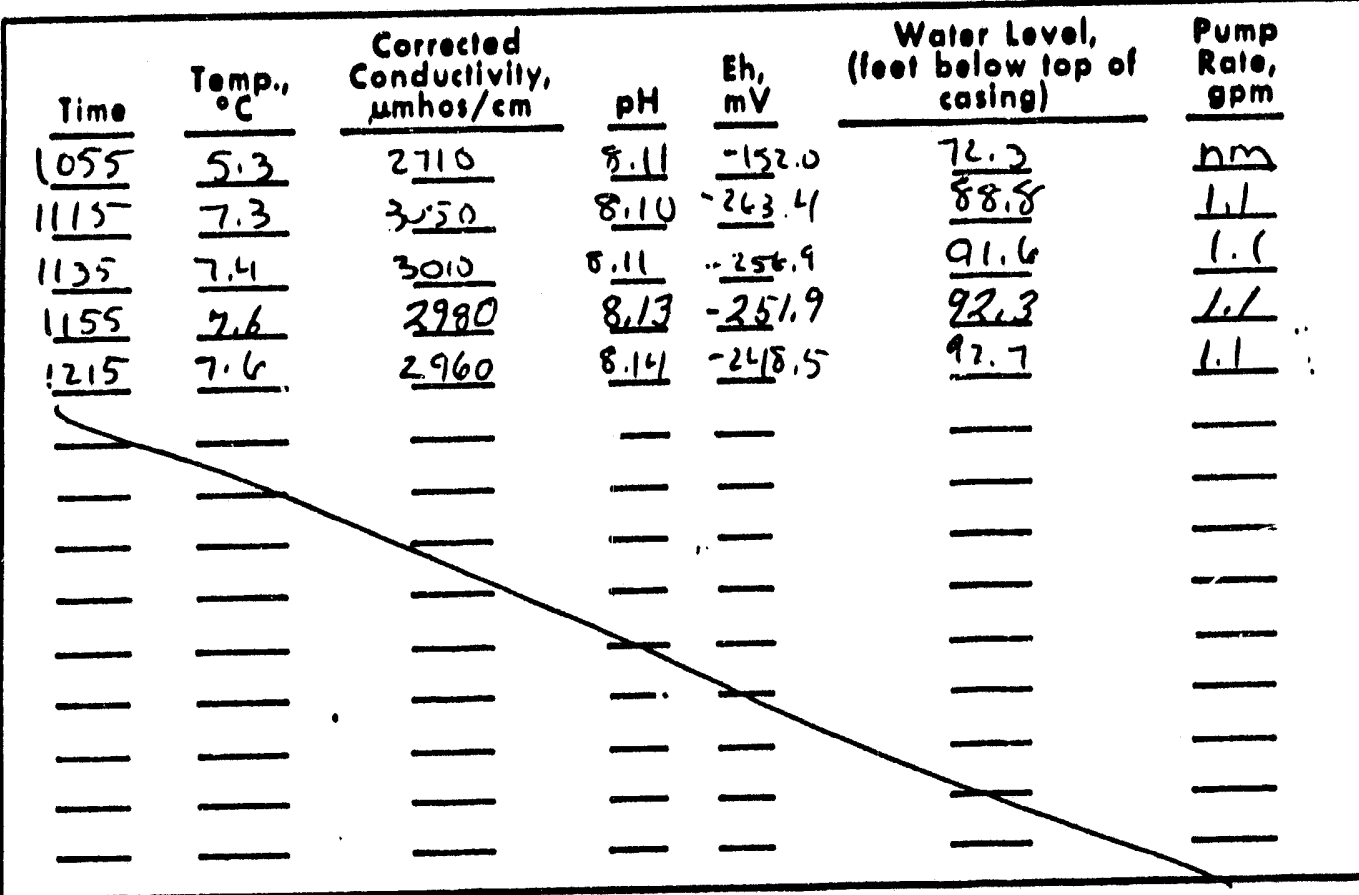

COMMENTS

Well NameEmw- Ila

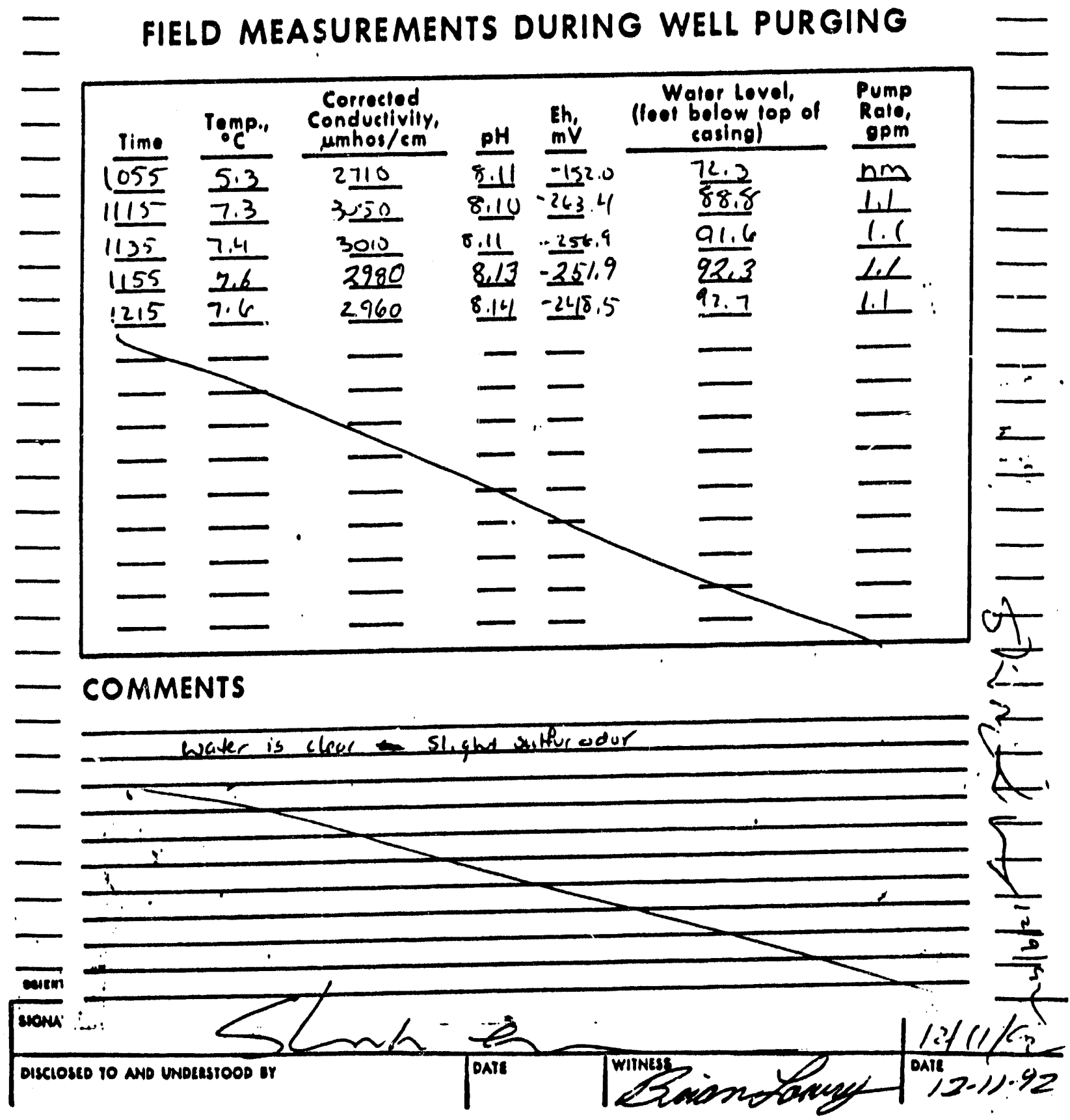


36 TITLE EMWU-1

PNOJKCT NO.05110300

Dook No. 078

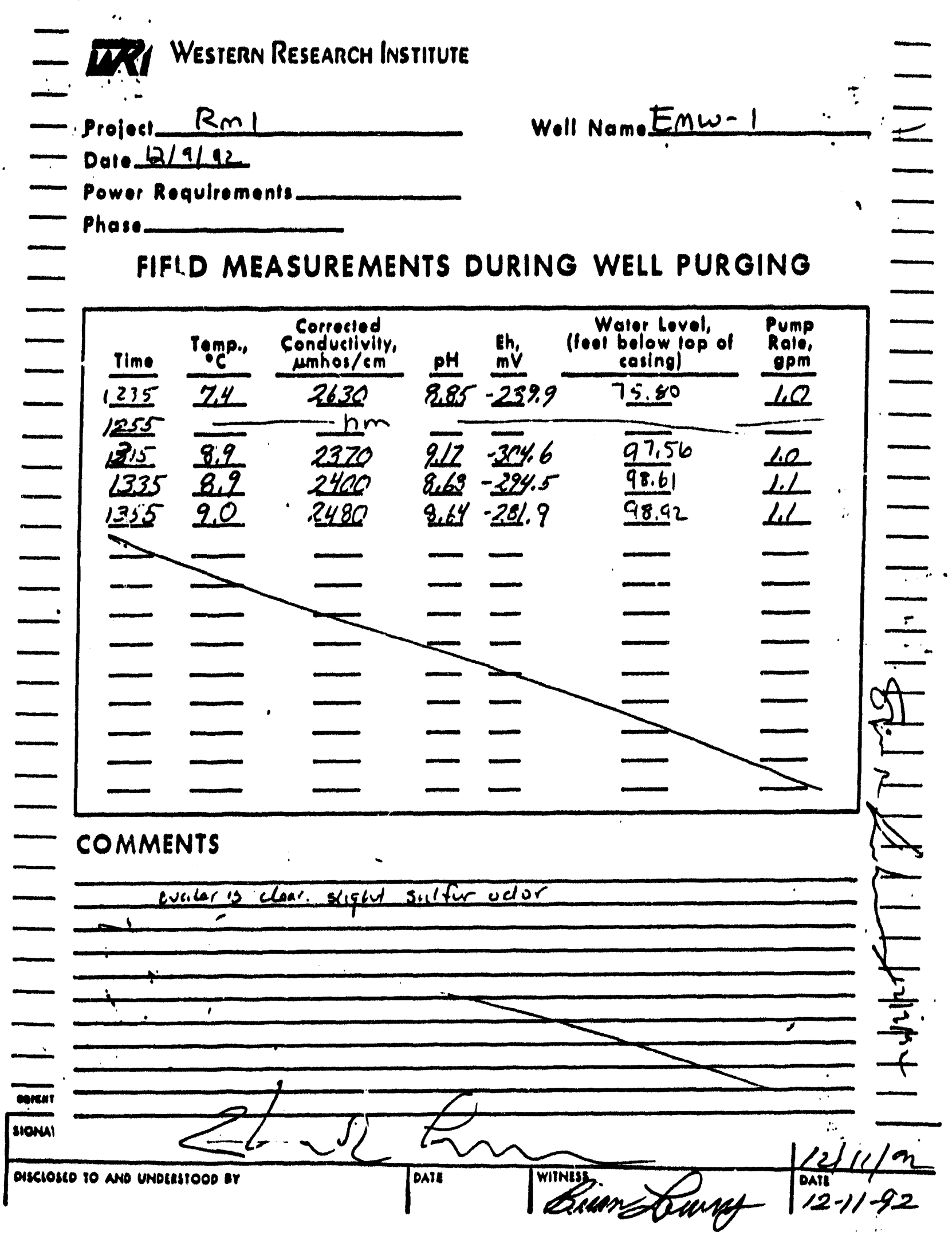


TITL EMW-1

PnOJECT NO.OSIIUNUO

DOOK NO.OIso

\section{Tril Western Reseanch Institute}

\section{WRI GROUNDWATER SAMPLING RECORD}

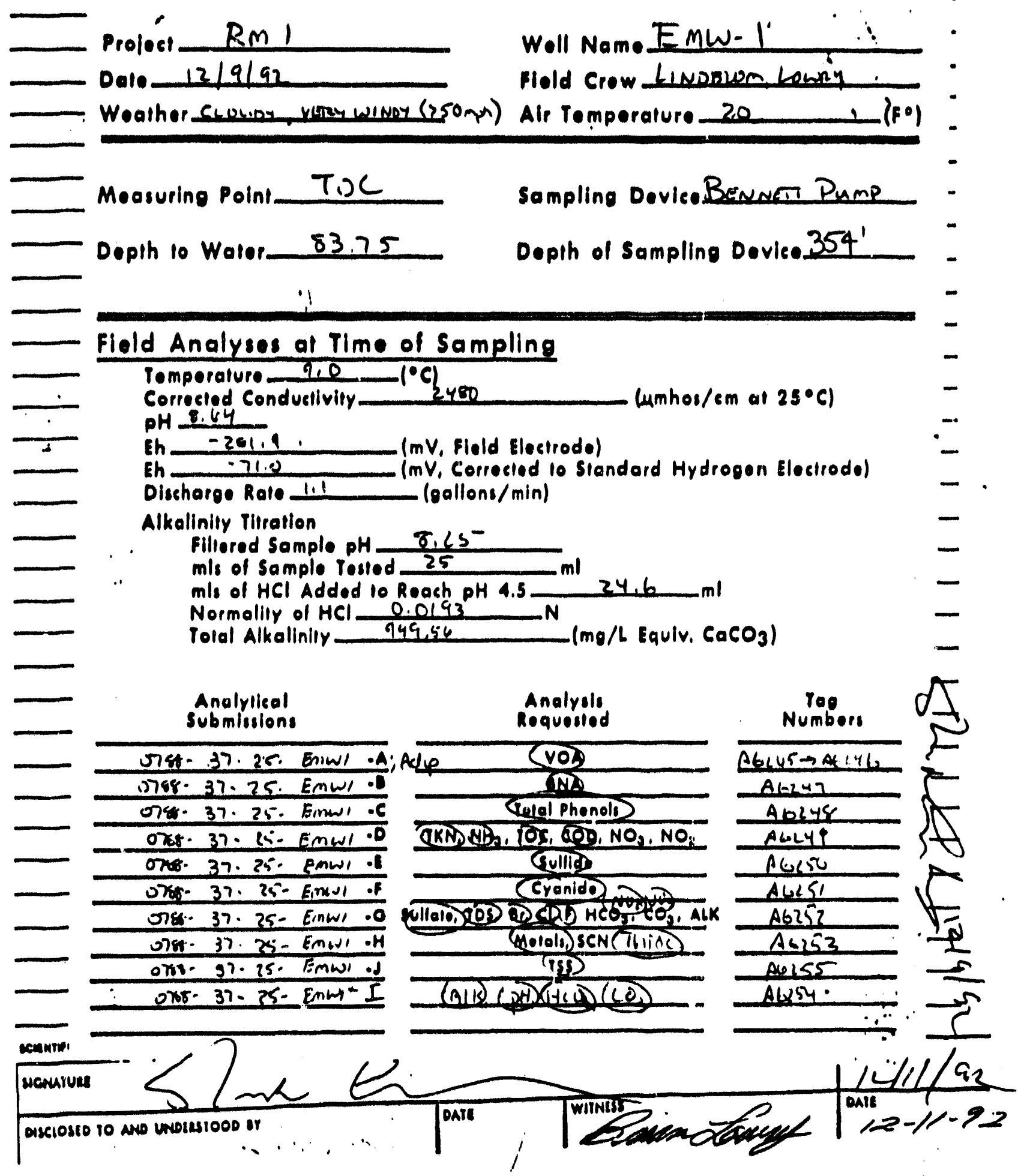



Emw-2
PROJECT NO. O5110300
DOOK NO. 0758

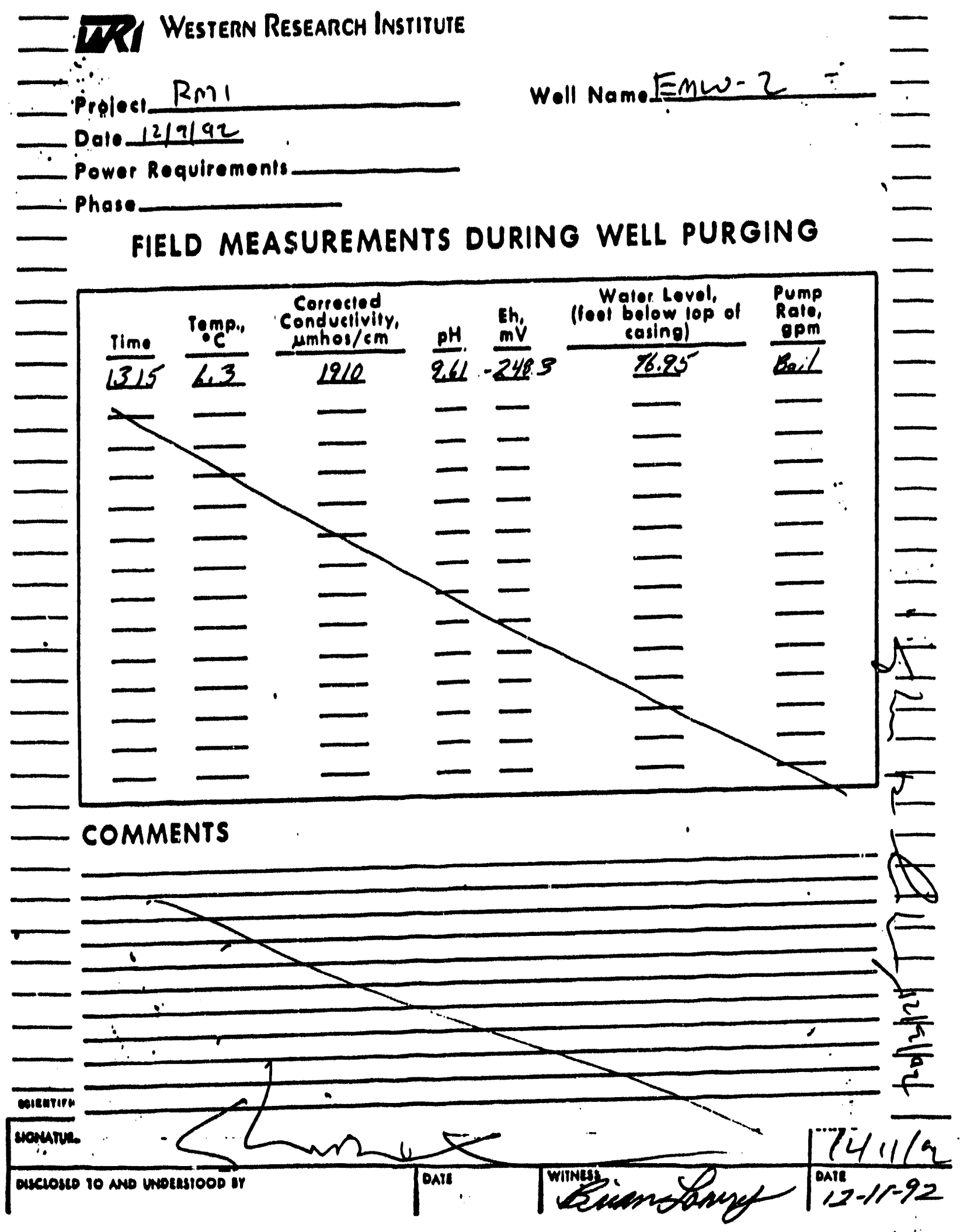


TITLE

Enw-2

PROdECT NO.US11 J3OU

BOOK NO. UT88

\section{wil Western Research Institute}

WRI GROUNDWATER SAMPLING RECORD $\because$

Projoel Rm

Dalo $1219 / 92$

Woather scumpy Veipy winOy (350)

W.ll Name Emw-2

Fiold Crow LindBLOA, Lowey

$\longrightarrow$

Woarhor clumpy vey wingt (a

Air Tomporafure $20 \quad 1$

Moasuring Point TOC

Sampling Dovico. TEFLON Baller

Dopth to Water_ 76.95

Dopth of Sampling Device 301

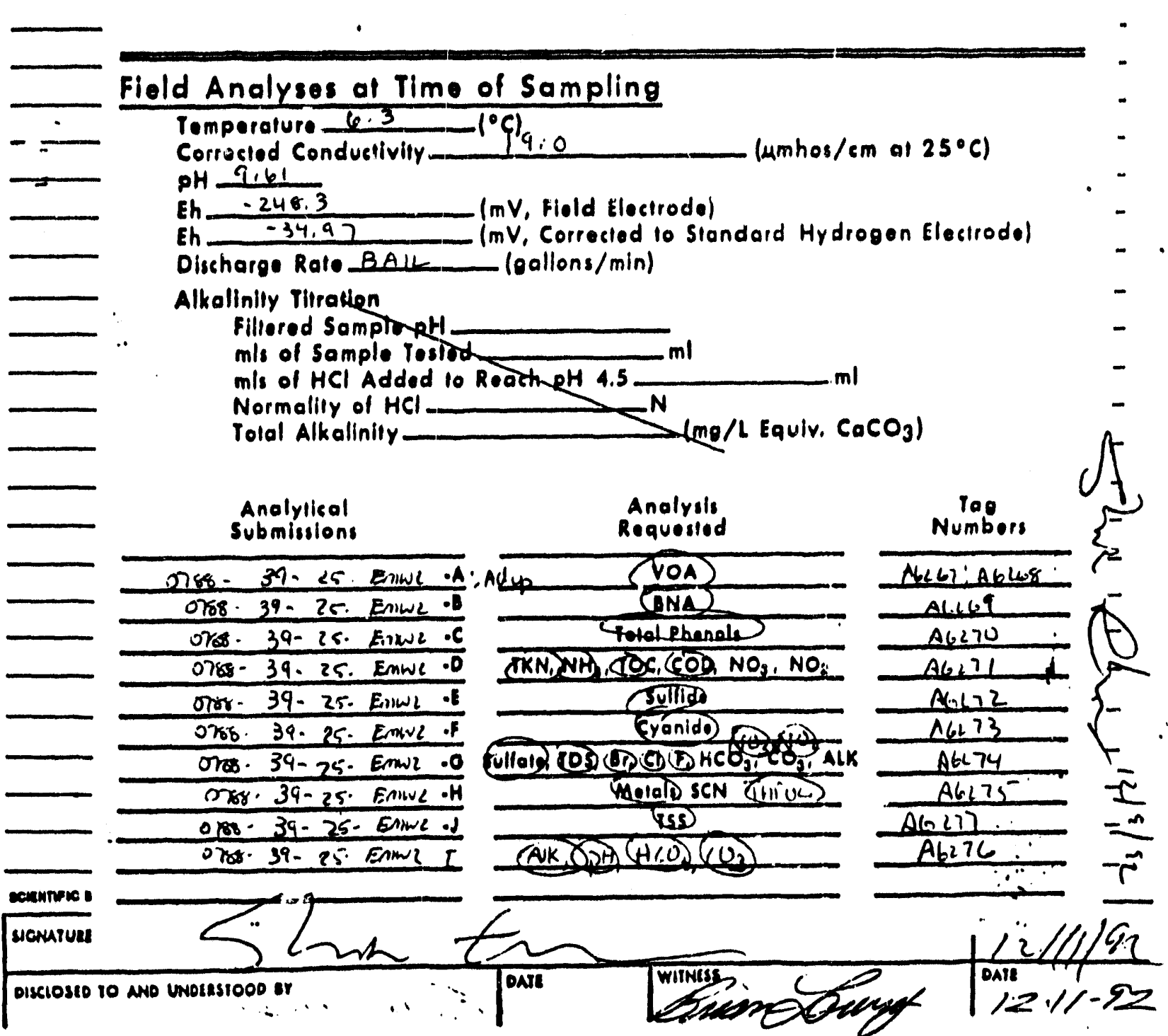




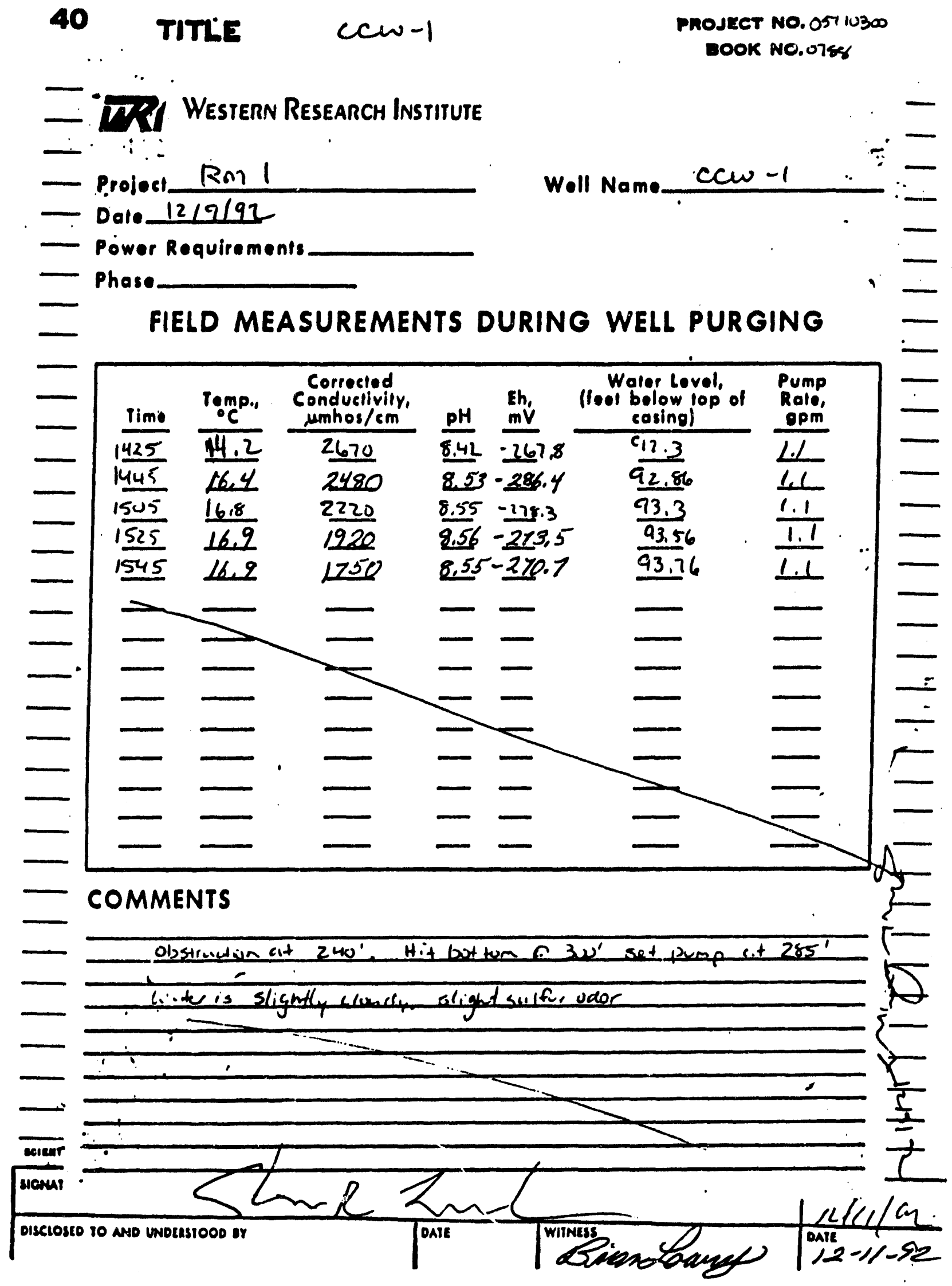




\section{WR! GROUNDWATER SAMPLING RECORD}

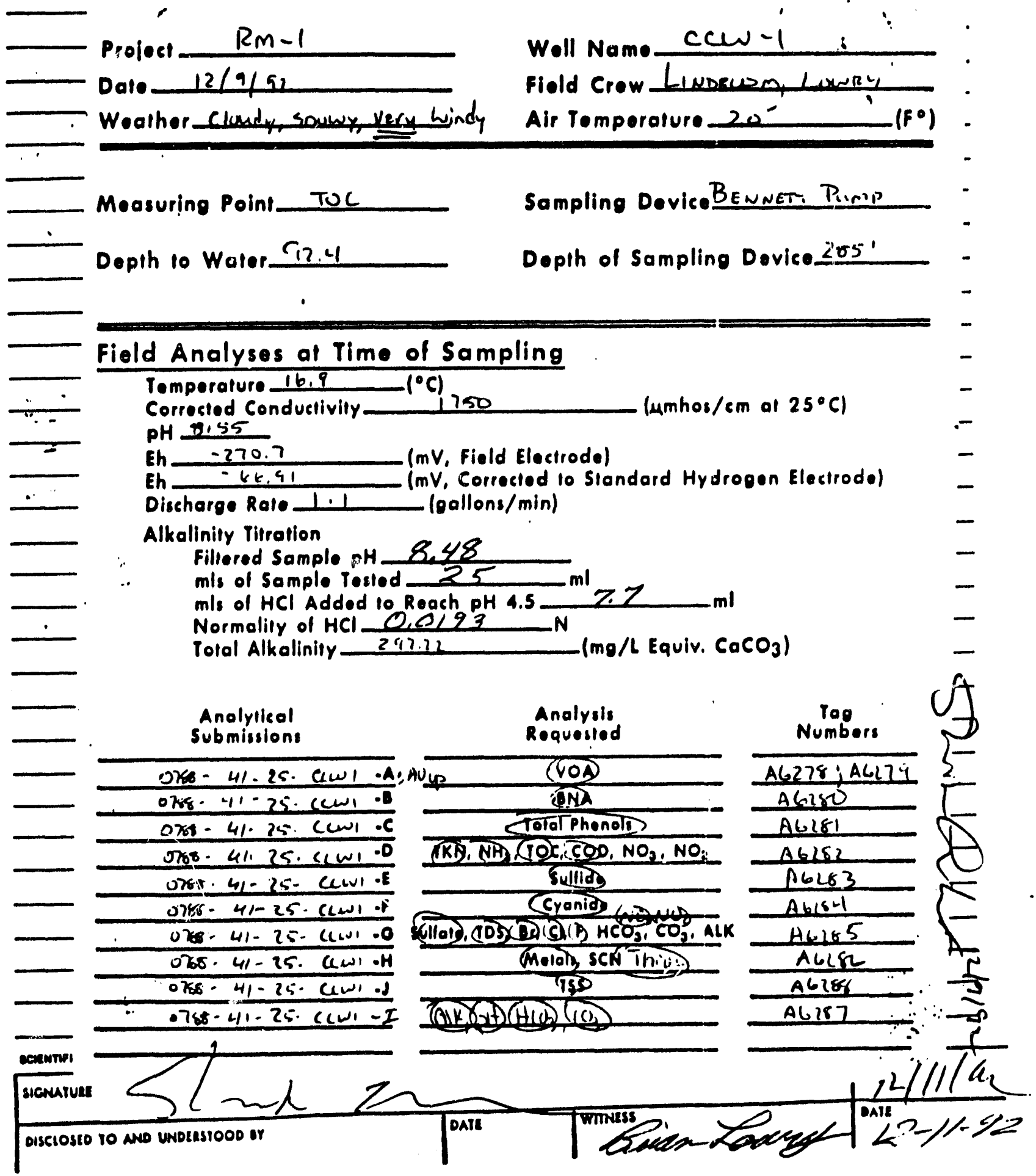




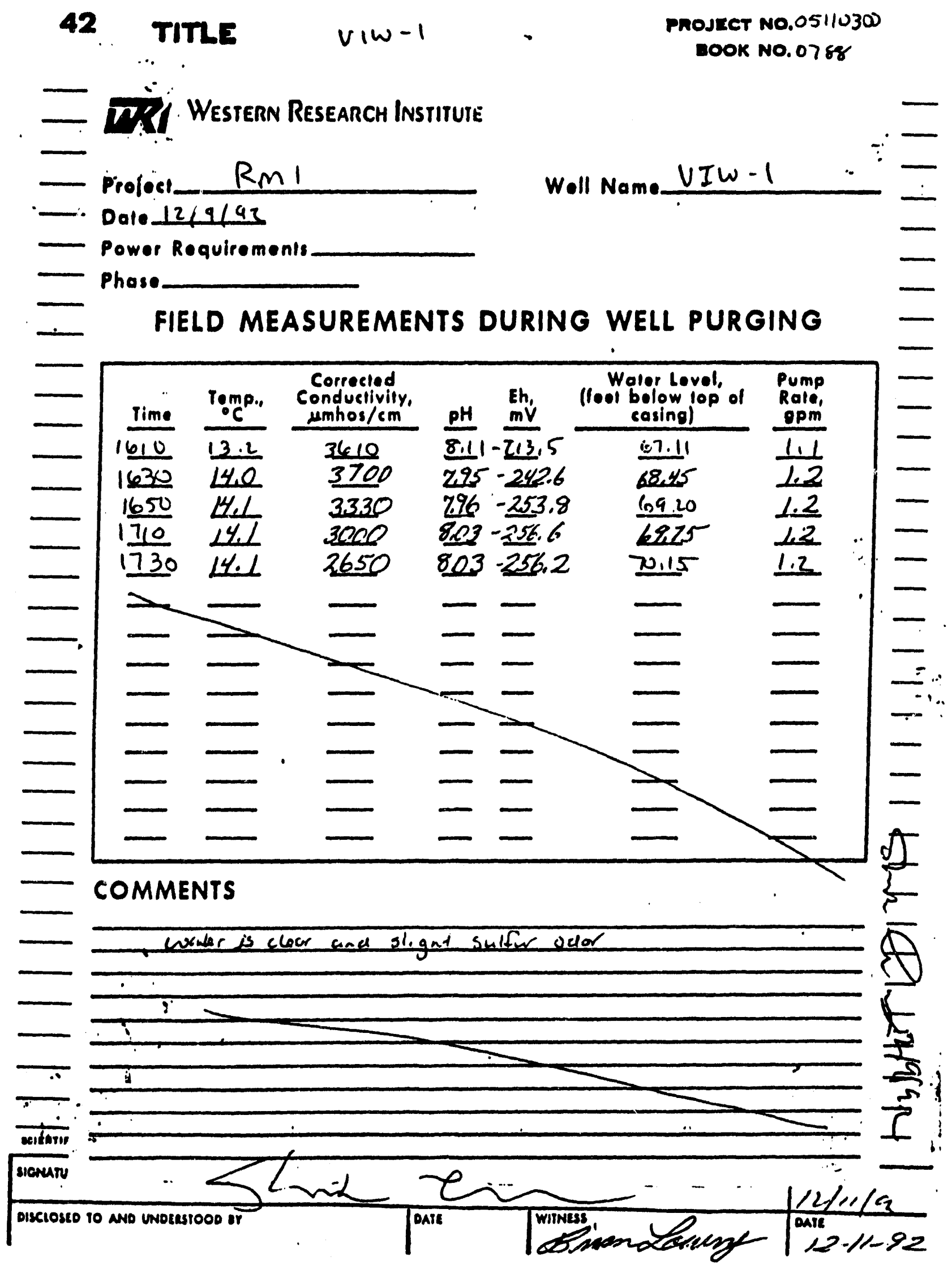


TITLI

- wil Western Research Institute

annk No 0788

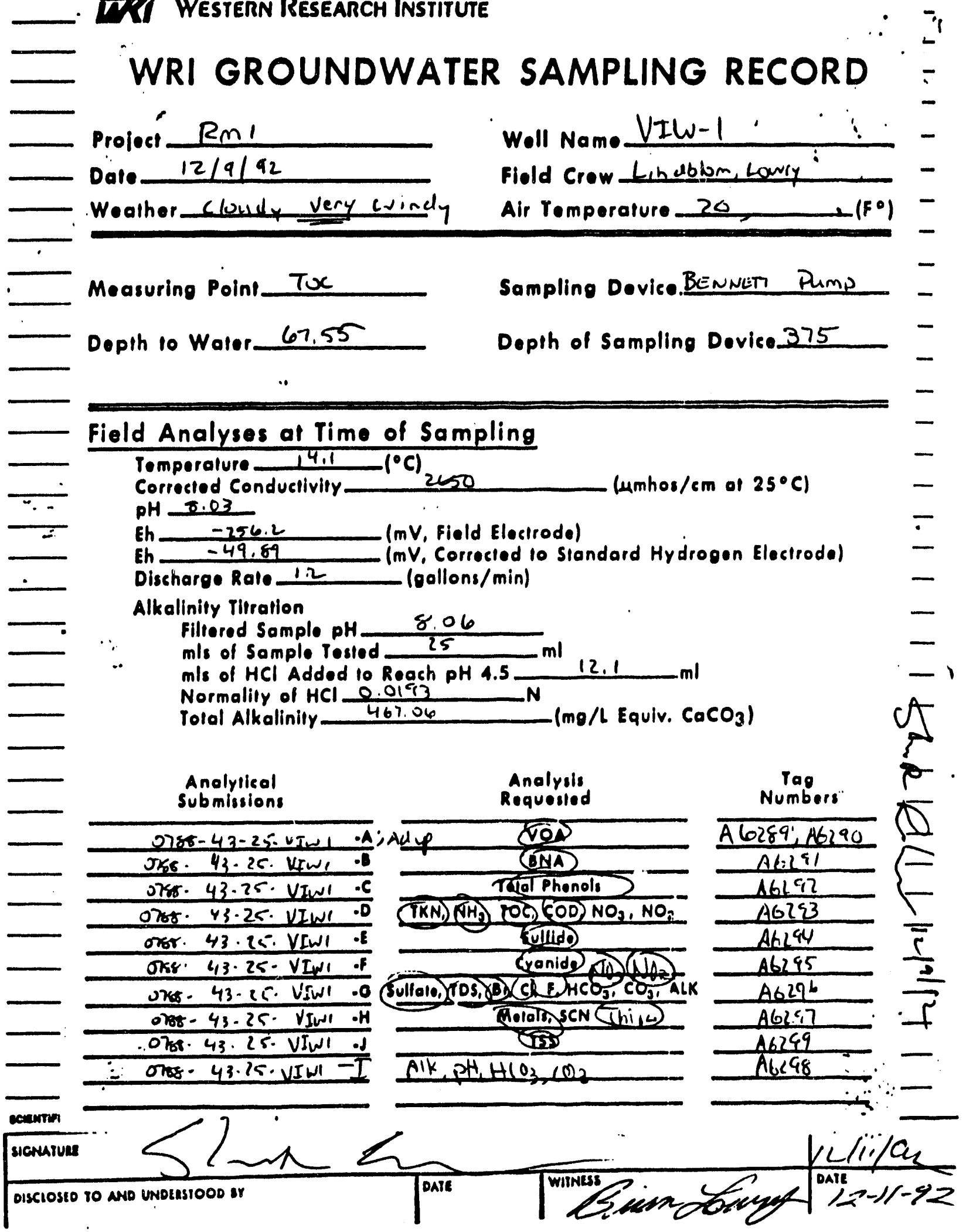




\section{GUDALRY}

During December 1992, groundwater was sampled at the site of the November $1987-F e b r u a r y ~ 1988$ Rocky Mountain 1 underground coal gasification teat near banna, Wyoming. The groundwater is near baseline condition. Data from the field measurements and analyses of samples are presented. Benzene concentrations in the groundwater are below analytical detection limits (<0.01 mg/L) for all wells, except concentrations of $0.016 \mathrm{mg} / \mathrm{L}$ and $0.013 \mathrm{mg} / \mathrm{L}$ in coal seam wells EMW-3 and EMW-1, respectively. 


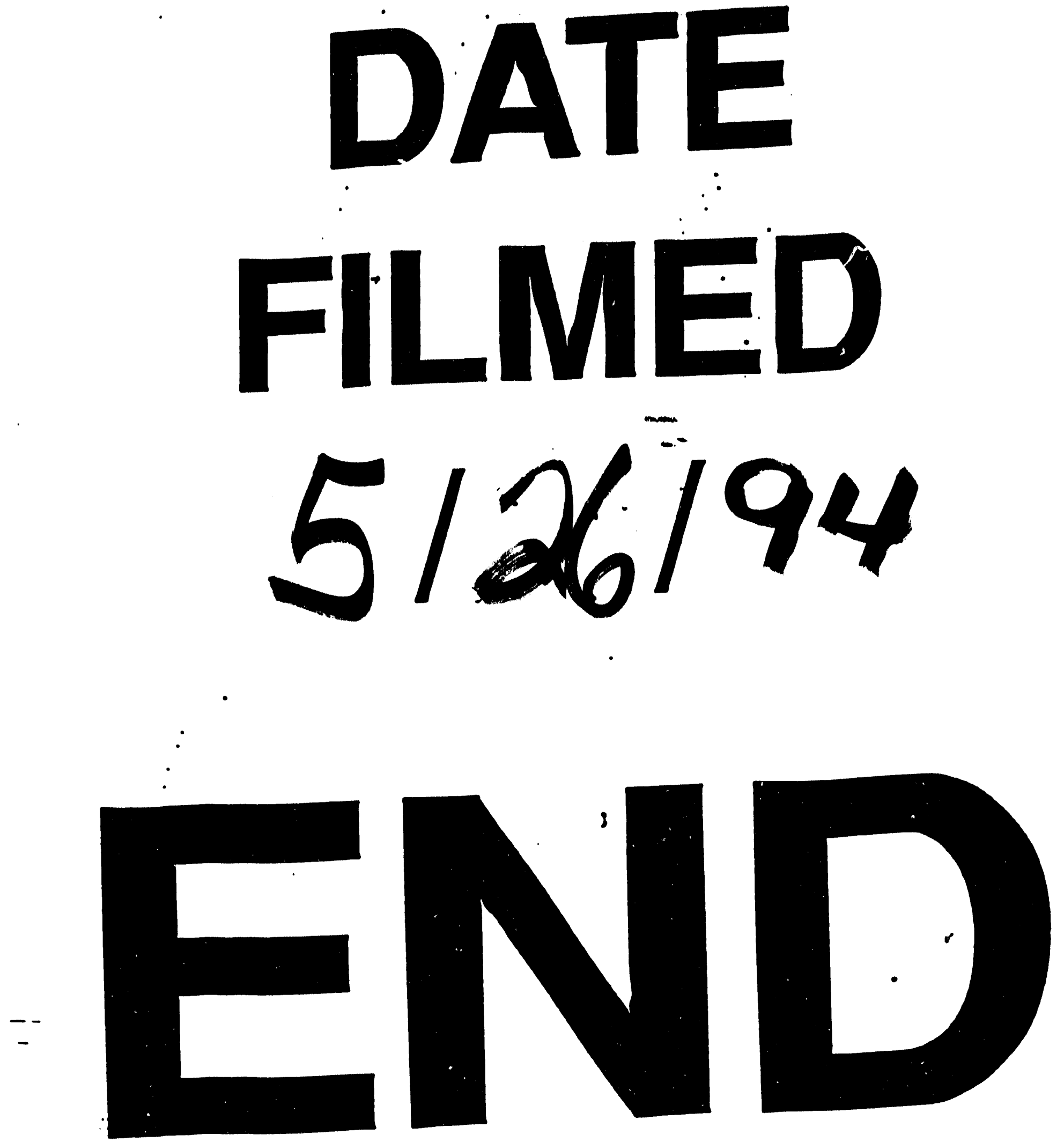


$$
\longrightarrow
$$

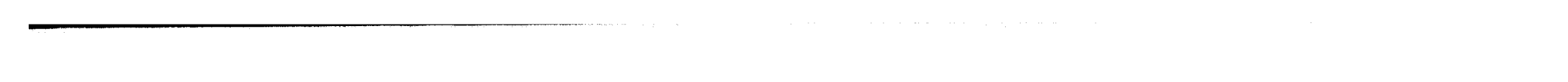
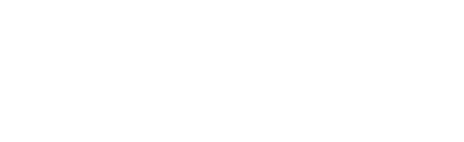